\title{
$\sigma$-Entangled linear orders and narrowness of products of Boolean algebras
}

by

Saharon Shelah (Jerusalem and New Brunswick, N.J.)

\begin{abstract}
We investigate $\sigma$-entangled linear orders and narrowness of Boolean algebras. We show existence of $\sigma$-entangled linear orders in many cardinals, and we build Boolean algebras with neither large chains nor large pies. We study the behavior of these notions in ultraproducts.
\end{abstract}

\section{Annotated content}

\section{Introduction}

1. Basic properties. We define $\mathrm{Ens}_{\sigma}, \sigma$-entangled (Definition 1.1); we give their basic properties (1.2) and the connection between those properties of linear orders and (the $\sigma$-completion of) the interval Boolean algebras (Definition 1.3) which they generate (1.5). We recall the definition of inc ${ }^{(+)}(B)$ (see 1.4) and we state its properties. Then we formulate the properties of linear orders required to have $\operatorname{inc}\left(B^{\sigma} / D\right)>(\operatorname{inc}(B))^{\sigma} / D$ (1.7).

2. Constructions for $\lambda=\lambda^{<\lambda}$. In 2.3, assuming $\lambda=2^{\mu}=\mu^{+}$(and $\diamond_{\lambda}$ which usually follows), we build some Boolean algebras derived from a tree, using a construction principle (see [Sh 405]). The tree is a $\lambda^{+}$-Aronszajn tree, the derived linear order is locally $\mu$-entangled (of cardinality $\lambda^{+}$). Next, in 2.5 , we force a subtree $T$ of ${ }^{\lambda} \geq \lambda$ of cardinality $\lambda^{+}$, the derived linear order is $\mu$-entangled (of cardinality $\lambda^{+}$). It provides an example of Boolean algebras $B_{\sigma}$ (for $\sigma<\mu$ ) with $\operatorname{inc}\left(B_{\sigma}\right)=\lambda$, inc $\left(\left(B_{\sigma}\right)^{\mu} / D\right)=\lambda^{+}$for each uniform ultrafilter $D$ on $\mu$.

3. Constructions related to pcf theory. We give sufficient conditions for $\operatorname{Ens}_{\sigma}(\lambda, \kappa)$ when $\lambda$ can be represented as $\operatorname{tcf}\left(\prod_{i} \lambda_{i} / D\right)$ with $\lambda_{i}>\max \operatorname{pcf}\left(\left\{\lambda_{j}: j<i\right\}\right)$ (see 3.1 ). If $2^{\kappa} \geq \sup _{i} \lambda_{i}$ (and more) we can get a $\sigma$-entangled linear order (3.2). Also we can utilize $\operatorname{Ens}_{\sigma}\left(\lambda_{i}, \kappa_{i}\right)$ (see $3.3,3.4$ ). Now relying on a generalization of " $\delta<\aleph_{\delta} \Rightarrow \operatorname{pp}\left(\aleph_{\delta}\right)<$ $\aleph_{|\delta|+4}$ ", we prove that if $\mu=\mu^{<\sigma}$ then for many $\theta \in\left[\mu, \aleph_{\mu^{+4}}\right)$ we have $\operatorname{Ens}_{\sigma}\left(\theta^{+}, \mu\right)$ and if $2^{\mu} \geq \aleph_{\mu^{+4}}$ also $\sigma$-entangled linear orders of cardinality $\theta^{+}$(see 3.6). Hence for each

1991 Mathematics Subject Classification: 03E05, 04A20, 06A07.

Partially supported by the Deutsche Forschungsgemeinschaft, Grant No. Ko 490/7-1. Publication 462. 
$\sigma$ for a class of successor cardinals there is a $\sigma$-entangled linear order of cardinality $\lambda^{+}$ (see 3.7).

4. Boolean algebras with neither pies nor chains. Refining results in Section 3 , we get Boolean algebras (again derived from trees $\bigcup_{i<\delta} \prod_{j<i} \lambda_{j}$ using $\lambda=\operatorname{tcf}\left(\prod_{i} \lambda_{i} / D\right)$, but not as interval Boolean algebras), which have neither large chains nor large pies. For this we need more on how $\lambda=\operatorname{tcf}\left(\prod_{i} \lambda_{i} / D\right)$.

5. More on entangledness. In 5.1, 5.4 we deal with cases $2^{<\lambda}<2^{\lambda}$. Then we get finer results from assumption on $\operatorname{pp}(\mu)$ 's, improving Section 3. We also deal with $\operatorname{pcf}(\mathfrak{a})$, defining $\operatorname{pcf}_{\kappa}^{\text {ex }}(\mathfrak{a})=\bigcap\{\operatorname{pcf}(\mathfrak{a} \backslash \mathfrak{b}): \mathfrak{b} \subseteq \mathfrak{a},|\mathfrak{b}|<\kappa\}$, proving for it the parallel of the old theorem and connecting it to entangledness, mainly: if each $\mu \in \mathfrak{a}$ is $(\lambda, \kappa, 2)$-inaccessible, then $\theta \in \operatorname{pcf}_{\kappa}^{\text {ex }}(\mathfrak{a}) \Rightarrow \operatorname{Ens}\left(\theta, 2^{\kappa}\right)$. We extract from the proof of [Sh 410, §4] on the existence of entangled linear orders a statement more relevant to pcf. We lastly prove: for a singular fix point $\mu$ and $\mu_{0}<\mu$ there is $\theta^{+} \in\left(\mu, \mathrm{pp}^{+}(\mu)\right)$ in which there is an entangled linear order of density $\in\left(\mu_{0}, \mu\right)$ (see $5.13(2,3)$ ).

6. Variants of entangledness in ultraproducts. We investigate what kinds of entangledness (and inc $(-) \leq \mu$ ) are preserved by ultraproducts (6.4). We also find that entangledness can be destroyed by ultrapowers with little connection to its structure, just its cardinality, for non-separative ultrafilters. So to show the possibility of $(\operatorname{inc}(B))^{\omega} / D>$ $\operatorname{inc}\left(B^{\omega} / D\right)$ it suffices to find $B=\mathrm{BA}_{\text {inter }}(\mathcal{I})$ such that $|B|>(\operatorname{inc}(B))^{\aleph_{0}}$.

0. Introduction. In the present paper we investigate $\sigma$-entangled linear orders and narrowness of Boolean algebras (if $B$ is the interval Boolean algebra of a linear order $\mathcal{I}$, then the algebra $B$ is narrow if and only if $\mathcal{I}$ is entangled). On entangled $=\aleph_{0}$-entangled ( $=$ narrow interval Boolean algebra) linear orders (Definition 1.1(4)) see Bonnet [Bo], Abraham-Shelah [AbSh 106], Abraham-Rubin-Shelah [ARSh 153], Bonnet-Shelah [BoSh 210], Todorčević [To] and [Sh 345, §4] [Sh 345b, §4], [Sh 355, 4.9-4.14], [Sh 410, §4].

We prove that for many cardinals $\lambda$ there is a $\sigma$-entangled linear order of cardinality $\lambda$ (see 3.7). For example, if $\lambda$ is a limit cardinal, $\lambda=\lambda^{<\sigma}$, $2^{\lambda}>\lambda^{+\lambda^{+4}}$ then for some singular cardinal $\mu \in\left[\lambda, \aleph_{\lambda^{+4}}\right)$ there is one in $\mu^{+}$. We also prove that for a class of cardinals $\lambda$, there is a Boolean algebra $B$ of cardinality $\lambda^{+}$with neither a chain of cardinality $\lambda^{+}$nor a pie $(=$set of pairwise incomparable elements) of cardinality $\lambda^{+}$(see 4.3).

Another focus is a problem of Monk [M1]: for a Boolean algebra $B$, let $\operatorname{inc}(B)$ be $\sup \{|X|: X \subseteq B$ is a pie $\}$. He asked: are there a Boolean algebra $B$, a cardinal $\sigma$ and an ultrafilter $D$ on $\sigma$ such that $\operatorname{inc}\left(B^{\sigma} / D\right)>$ $\operatorname{inc}(B))^{\sigma} / D$, and we may ask whether this holds for $\sigma$ but for no smaller $\sigma^{\prime}<\sigma$. Now, if $\mathcal{I}$ is a $\sigma$-entangled linear order of cardinality $\lambda^{+}, \lambda^{\sigma}=\lambda$ then we get examples: the interval Boolean algebra $B$ of $\mathcal{I}$ satisfies $\operatorname{inc}(B)=\lambda$ (hence $(\operatorname{inc}(B))^{\sigma} / D=\lambda$ ), but in the cases we construct $\mathcal{I}$, we get inc $\left(B^{\sigma} / D\right)$ $=\lambda^{+}$for any uniform ultrafilter $D$ on $\sigma$ (on sufficiency see 1.7; on existence see $2.3(3), 3.2,3.6(3))$. Similarly for the entangledness of a linear order. Unfortunately, though we know that there are $\sigma$-entangled linear orders of cardinality $\lambda^{+}$for many cardinals $\lambda$ (as needed), we do not know this for 
cardinals $\lambda$ satisfying $\lambda=\lambda^{\sigma}$ (even $\lambda^{\aleph_{0}}=\lambda$ ), and $\lambda<\lambda^{\sigma}$ implies usually $(\operatorname{inc}(B))^{\sigma} / D \geq \lambda^{+}$. Still, the unresolved case requires quite peculiar cardinal arithmetic (everywhere): "usually" $2^{\lambda}$ is not so large in the aleph sequence, and there are additional strong restrictions on the power structure in $\mathbf{V}$. For instance, for every $\mu$,

$$
\mu^{\sigma}=\mu \Rightarrow 2^{\mu}<\aleph_{\mu^{+4}}
$$

and

$$
\mu \text { is strong limit of cofinality }>\sigma \Rightarrow 2^{\mu}<\mu^{+\mu} \&(\exists \chi)\left(\chi<\chi^{\sigma}=2^{\mu}\right)
$$

and

$$
\mu \geq \beth_{\omega} \Rightarrow 2^{\mu}>\mu^{+} .
$$

To make the paper more self-contained we give fully the straight generalizations of [Sh 345], [Sh 355] and [Sh 410]. The research is continued in Magidor-Shelah [MgSh 433], Shafir-Shelah [SaSh 553], Rosłanowski-Shelah [RoSh 534], [RoSh 599], and lately [Sh 620].

We thank Andrzej Rosłanowski and Opher Shafir for reading, correcting, pointing out various flaws and writing down significant expansions.

Notation. Our notation is rather standard. We will keep the following rules for our notation:

(1) $\alpha, \beta, \gamma, \delta, \xi, \zeta, i, j \ldots$ will denote ordinals,

(2) $\kappa, \lambda, \mu, \sigma, \ldots$ will stand for cardinal numbers,

(3) a bar above a name indicates that the object is a sequence; usually $\bar{X}$ will be $\left\langle X_{i}: i<\lg (\bar{X})\right\rangle$, where $\lg (\bar{X})$ denotes the length of $\bar{X}$,

(4) for two sequences $\eta, \nu$ we write $\nu \triangleleft \eta$ whenever $\nu$ is a proper initial segment of $\eta$, and $\nu \unlhd \eta$ when either $\nu \triangleleft \eta$ or $\nu=\eta$.

For a set $A$ of ordinals with no last element, $J_{A}^{\mathrm{bd}}$ is the ideal of bounded subsets of $A$.

1. Basic properties. In this section we formulate basic definitions and prove fundamental dependencies between the notions we introduce.

DeFinition 1.1. Let $\lambda, \mu, \kappa, \sigma$ be cardinal numbers.

(1) A sequence $\overline{\mathcal{I}}=\left\langle\mathcal{I}_{\varepsilon}: \varepsilon<\kappa\right\rangle$ of linear orders is $(\mu, \sigma)$-entangled if

$(\circledast) \quad$ for any disjoint subsets $u, v$ of $\kappa$ such that $|u \cup v|<1+\sigma$ and sequences $\left\langle t_{\alpha}^{\varepsilon}: \alpha<\mu\right\rangle$ of pairwise distinct elements of $\mathcal{I}_{\varepsilon}($ for $\varepsilon \in u \cup v$ ), there are $\alpha<\beta<\mu$ such that

$$
\varepsilon \in u \Rightarrow t_{\alpha}^{\varepsilon}<_{\mathcal{I}_{\varepsilon}} t_{\beta}^{\varepsilon} \quad \text { and } \quad \varepsilon \in v \Rightarrow t_{\alpha}^{\varepsilon}>_{\mathcal{I}_{\varepsilon}} t_{\beta}^{\varepsilon} .
$$

$\operatorname{Ens}(\lambda, \mu, \kappa, \sigma)=\operatorname{Ens}_{\sigma}(\lambda, \mu, \kappa)$ means: there is a $(\mu, \sigma)$-entangled sequence $\overline{\mathcal{I}}=\left\langle\mathcal{I}_{\varepsilon}: \varepsilon<\kappa\right\rangle$ of linear orders, each of cardinality $\lambda$. 
(2) If we omit $\mu$, this means $\lambda=\mu$ (i.e. $\left|\mathcal{I}_{\varepsilon}\right|=\mu$ ), if we omit $\sigma$ it means $\sigma=\aleph_{0}$.

(3) A linear order $\mathcal{I}$ is $(\mu, \sigma)$-entangled if ( $\mathcal{I}$ has cardinality $\geq \mu$ and) for every $\varepsilon(*)<\sigma$ and a partition $(u, v)$ of $\varepsilon(*)$ and pairwise distinct $t_{\alpha}^{\varepsilon} \in \mathcal{I}$ (for $\varepsilon \in u \cup v$ and $\alpha<\mu$ ), there are $\alpha<\beta<\mu$ such that

$(\oplus) \quad$ for each $\varepsilon<\varepsilon(*)$ we have

$$
\varepsilon \in u \Rightarrow t_{\alpha}^{\varepsilon}<_{\mathcal{I}} t_{\beta}^{\varepsilon} \quad \text { and } \quad \varepsilon \in v \Rightarrow t_{\alpha}^{\varepsilon}>_{\mathcal{I}} t_{\beta}^{\varepsilon} .
$$

(4) We omit $\mu$ if $|\mathcal{I}|=\mu$ (and so we write " $\mathcal{I}$ is $\sigma$-entangled" instead of "I $\mathcal{I}$ is $(|\mathcal{I}|, \sigma)$-entangled"); we also omit $\sigma$ if it is $\aleph_{0}$. if

(5) A sequence $\left\langle\mathcal{I}_{\zeta}: \zeta<\gamma\right\rangle$ of linear orders is strongly $\left(\mu, \sigma, \sigma^{\prime}\right)$-entangled

(a) each $\mathcal{I}_{\zeta}$ is of cardinality $\geq \mu$,

(b) if $u, v$ are disjoint subsets of $\gamma,|u \cup v|<1+\sigma, \xi(\varepsilon)<\sigma^{\prime}$ for $\varepsilon \in u \cup v$ and $t_{\varepsilon, \xi}^{\alpha} \in \mathcal{I}_{\varepsilon}$ (for $\alpha<\mu, \varepsilon \in u \cup v, \xi<\xi(\varepsilon)$ ) are such that

$$
(\forall \varepsilon \in u \cup v)(\forall \xi, \zeta<\xi(\varepsilon))(\forall \alpha<\beta<\mu)\left(t_{\varepsilon, \xi}^{\alpha} \neq t_{\varepsilon, \zeta}^{\beta}\right)
$$

then for some $\alpha<\beta<\mu$ we have:

$$
\begin{aligned}
& \varepsilon \in u \Rightarrow(\forall \xi<\xi(\varepsilon))\left(t_{\varepsilon, \xi}^{\alpha}<t_{\varepsilon, \xi}^{\beta}\right), \\
& \varepsilon \in v \Rightarrow(\forall \xi<\xi(\varepsilon))\left(t_{\varepsilon, \xi}^{\beta}<t_{\varepsilon, \xi}^{\alpha}\right) .
\end{aligned}
$$

Proposition 1.2. (1) Assume $\lambda \geq \lambda_{1} \geq \mu_{1} \geq \mu, \kappa_{1} \leq \kappa$ and $\sigma_{1} \leq \sigma$. Then $\operatorname{Ens}_{\sigma}(\lambda, \mu, \kappa)$ implies $\operatorname{Ens}_{\sigma_{1}}\left(\lambda_{1}, \mu_{1}, \kappa_{1}\right)$.

(2) If $\mathcal{I}$ is a $(\mu, \sigma)$-entangled linear order, $\mathcal{J} \subseteq \mathcal{I}$, and $|\mathcal{I}| \geq|\mathcal{J}| \geq \mu_{1} \geq$ $\mu, \sigma_{1} \leq \sigma$ then $\mathcal{J}$ is $\left(\mu_{1}, \sigma_{1}\right)$-entangled.

(3) If a linear order $\mathcal{I}$ has density $\chi, \chi^{<\sigma}<\mu, \mu=\operatorname{cf}(\mu)$ and $\sigma \geq 2$ then in Definition 1.1(3) of "I $\mathcal{I}$ is $(\mu, \sigma)$-entangled" we can add to the assumptions (๑) there is a sequence $\left\langle\left[a_{\varepsilon}, b_{\varepsilon}\right]: \varepsilon<\varepsilon(*)\right\rangle$ of pairwise disjoint intervals of $\mathcal{I}$ such that $t_{\alpha}^{\varepsilon} \in\left(a_{\varepsilon}, b_{\varepsilon}\right)$.

(4) Moreover, if a linear order $\mathcal{I}$ has density $\chi$ and $\chi^{<\sigma}<\mu=\operatorname{cf}(\mu)$, then for each $\varepsilon(*)<\sigma$ and sequences $\bar{t}_{\alpha}=\left\langle t_{\alpha}^{\varepsilon}: \varepsilon<\varepsilon(*)\right\rangle \subseteq \mathcal{I}($ for $\alpha<\mu)$ such that $\varepsilon \neq \zeta \Rightarrow t_{\alpha}^{\varepsilon} \neq t_{\alpha}^{\zeta}$, there are $A \subseteq \mu$ with $|A|=\mu$ and a sequence $\left\langle\left[a_{\varepsilon}, b_{\varepsilon}\right]: \varepsilon<\varepsilon(*)\right\rangle$ of pairwise disjoint intervals of $\mathcal{I}$ such that for each $\varepsilon<\varepsilon(*)$, either

$$
(\forall \alpha \in A)\left(t_{\alpha}^{\varepsilon} \in\left(a_{\varepsilon}, b_{\varepsilon}\right)\right) \quad \text { or } \quad(\forall \alpha \in A)\left(t_{\alpha}^{\varepsilon}=a_{\varepsilon}\right) .
$$

(5) If $\sigma \geq 2$ and a linear order $\mathcal{I}$ is $(\mu, \sigma)$-entangled then $\mathcal{I}$ has density $<\mu$.

(6) If there exists a $(\mu, \sigma)$-entangled linear order of size $\lambda$ then we have $\operatorname{Ens}_{\sigma}(\lambda, \mu, \lambda)$. 
(7) In Definition 1.1(3), if $\sigma$ is infinite, we can weaken " $\alpha<\beta<\mu$ " to " $\alpha \neq \beta, \alpha<\mu, \beta<\mu$ ".

(8) If there is a $(\mu, \sigma)$-entangled linear order of size $\lambda$ and $(*)_{\kappa}$ below holds then $\operatorname{Ens}_{\sigma}(\lambda, \mu, \kappa)$, where:

$(*)_{\kappa} \quad$ one of the following holds true:

( $\alpha) \kappa=\mu^{+}$and if $\lambda=\mu$ then $\operatorname{cf}(\mu) \geq \sigma$,

$(\beta)$ there are $A_{i} \subseteq \lambda$ for $i<\kappa,\left|A_{i}\right|=\lambda$ such that $i \neq j \Rightarrow\left|A_{i} \cap A_{j}\right|<$ $\mu$ and $\operatorname{cf}(\mu) \geq \sigma$,

$(\gamma)$ there are $A_{i} \subseteq \lambda$ for $i<\kappa,\left|A_{i}\right|=\lambda$ such that $\sup \left\{\left|A_{i} \cap A_{j}\right|\right.$ : $i<j<\kappa\}<\mu$.

Proof. (1), (2) are left to the reader.

(3) Clearly the new definition is weaker, so we shall prove that the one from 1.1(3) holds assuming the one from 1.2(3). Let $\mathcal{J} \subseteq \mathcal{I}$ be dense in $\mathcal{I}$ and $|\mathcal{J}| \leq \chi$. Thus for each $a, b \in \mathcal{I}$ with $a<_{\mathcal{I}} b$, there exists $s \in \mathcal{J}$ such that $a \leq_{\mathcal{I}} s \leq_{\mathcal{I}} b$.

Suppose that $\varepsilon(*), u, v$ and $\left\langle t_{\alpha}^{\varepsilon}: \varepsilon<\varepsilon(*), \alpha<\mu\right\rangle$ are as in 1.1(3). For each $\varepsilon, \zeta<\varepsilon(*)$ and $\alpha<\mu$ such that $t_{\alpha}^{\varepsilon}<t_{\alpha}^{\zeta}$ there exists $s_{\alpha}^{\varepsilon, \zeta} \in \mathcal{J}$ such that $t_{\alpha}^{\varepsilon} \leq s_{\alpha}^{\varepsilon, \zeta} \leq t_{\alpha}^{\zeta}$ (and at least one inequality is strict). Define functions $h_{0}$, $h_{1}, h_{2}, h_{3}$ on $\mu$ by

$$
\begin{aligned}
& h_{0}(\alpha)=\left\{\langle\varepsilon, \zeta\rangle: \varepsilon, \zeta<\varepsilon(*) \text { and } t_{\alpha}^{\varepsilon}<t_{\alpha}^{\zeta}\right\}, \\
& h_{1}(\alpha)=\left\langle s_{\alpha}^{\varepsilon, \zeta}:\langle\varepsilon, \zeta\rangle \in h_{0}(\alpha)\right\rangle, \\
& h_{2}(\alpha)=\left\langle\left\langle\varepsilon, \zeta, \xi, \operatorname{TV}\left(t_{\alpha}^{\xi}=s_{\alpha}^{\varepsilon, \zeta}\right)\right\rangle:\langle\varepsilon, \zeta\rangle \in h_{0}(\alpha), \xi<\varepsilon(*)\right\rangle, \\
& h_{3}(\alpha)=\left\langle\left\langle\varepsilon, \zeta, \xi, \operatorname{TV}\left(t_{\alpha}^{\xi}<s_{\alpha}^{\varepsilon, \zeta}\right)\right\rangle:\langle\varepsilon, \zeta\rangle \in h_{0}(\alpha), \xi<\varepsilon(*)\right\rangle,
\end{aligned}
$$

where $\operatorname{TV}(-)$ is the truth value of - . Now, for each $l<4$, $\operatorname{dom}\left(h_{l}\right)=\mu$ and $\left|\operatorname{rang}\left(h_{l}\right)\right| \leq|\mathcal{J}|^{|\varepsilon(*)|^{3}} \leq \chi^{<\sigma}<\mu$. Since $\operatorname{cf}(\mu)=\mu$, there exists $A \in[\mu]^{\mu}$ such that the restrictions $h_{l} \uparrow A$ are constant for $l=0,1,2,3$.

So let $s_{\alpha}^{\varepsilon, \zeta}=s^{\varepsilon, \zeta}$ for $\alpha \in A$. As the $t_{\alpha}^{\varepsilon}$ 's were pairwise distinct (for each $\varepsilon)$ we conclude

$$
(\alpha \in A \& \varepsilon<\varepsilon(*)) \Rightarrow t_{\alpha}^{\varepsilon} \notin\left\{s^{\xi, \zeta}:\langle\xi, \zeta\rangle \in h_{0}(\alpha)\right\} .
$$

For $\varepsilon<\varepsilon(*)$ define

$$
\mathcal{I}_{\varepsilon}=\left\{t \in \mathcal{I}: \text { for every } \zeta, \xi<\varepsilon(*) \text { such that } s^{\xi, \zeta}\right. \text { is well defined and }
$$

$$
\begin{aligned}
& \text { for every (三 some) } \alpha \in A \text { we have } \\
& \left.\left[t \leq s^{\xi, \zeta} \Leftrightarrow t_{\alpha}^{\varepsilon} \leq s^{\xi, \zeta}\right] \text { and }\left[t \geq s^{\xi, \zeta} \Leftrightarrow t_{\alpha}^{\varepsilon} \geq s^{\xi, \zeta}\right]\right\} .
\end{aligned}
$$

Note that the value of $\alpha$ is immaterial.

Now, clearly $\mathcal{I}_{\varepsilon}$ does not have cofinality $>\chi$ (as $\mathcal{I}$ has no monotonic sequence of length $\geq \chi^{+}$; remember $\mathcal{I}$ has density $\left.\leq \chi\right)$. Hence we find an unbounded well ordered subset $\mathcal{J}_{\varepsilon}^{+} \subseteq \mathcal{I}_{\varepsilon}$ with $\left|\mathcal{J}_{\varepsilon}^{+}\right| \leq \chi$. Similarly there 
is an anti-well ordered $\mathcal{J}_{\varepsilon}^{-} \subseteq \mathcal{I}_{\varepsilon}$ with $\left|\mathcal{J}_{\varepsilon}^{-}\right| \leq \chi$ which is unbounded from below (in $\mathcal{I}_{\varepsilon}$ ). Let $\mathcal{J}^{*}=\bigcup_{\varepsilon<\varepsilon(*)}\left(\mathcal{J}_{\varepsilon}^{+} \cup \mathcal{J}_{\varepsilon}^{-}\right)$. Again, for some set $A^{\prime} \subseteq A$ of size $\mu$, the Dedekind cut which $t_{\alpha}^{\varepsilon}$ realizes in $\mathcal{J}^{*}$ does not depend on $\alpha$ for $\alpha \in A^{\prime}$, and $t_{\alpha}^{\varepsilon} \notin \mathcal{J}^{*}$. Now we can easily choose $\left(a_{\varepsilon}, b_{\varepsilon}\right): a_{\varepsilon}$ is any member of $\mathcal{J}_{\varepsilon}^{-}$which is $<t_{\alpha}^{\varepsilon}$ for all $\alpha \in A^{\prime}$ and $b_{\varepsilon}$ is any member of $\mathcal{J}_{\varepsilon}^{+}$which is $>t_{\alpha}^{\varepsilon}$ for all $\alpha \in A^{\prime}$.

(4) Included in the proof of 1.2(3).

(5) By 1.2(2), without loss of generality $\sigma=2$. Suppose that $\mathcal{I}$ has density at least $\mu$. By induction on $\alpha<\mu$ we try to choose $t_{\alpha}^{0}, t_{\alpha}^{1}$ such that

(i) $t_{\alpha}^{0}<t_{\alpha}^{1}$,

(ii) $t_{\alpha}^{0}, t_{\alpha}^{1} \notin\left\{t_{\beta}^{0}, t_{\beta}^{1}: \beta<\alpha\right\}$,

(iii) $(\forall \beta<\alpha)(\forall l \in\{0,1\})\left(t_{\alpha}^{0}<t_{\beta}^{l} \Leftrightarrow t_{\alpha}^{1}<t_{\beta}^{l}\right)$.

Continue to define for as long as possible. There are two possible outcomes.

Outcome (a): One gets stuck at some $\alpha<\mu$. Let $\mathcal{J}=\left\{t_{\beta}^{0}, t_{\beta}^{1}: \beta<\alpha\right\}$. Then

$$
\left(\forall t^{0}<t^{1} \in \mathcal{I} \backslash \mathcal{J}\right)(\exists s \in \mathcal{J})\left(t^{0}<s \Leftrightarrow \neg\left[t^{1}<s\right]\right) .
$$

Since $t^{0}, t^{1} \notin \mathcal{J}$, it follows that $t^{0}<s<t^{1}$. So $\mathcal{J}$ is dense in $\mathcal{I}$ and is of cardinality $2|\alpha|<\mu-$ a contradiction.

Outcome (b): One can define $t_{\alpha}^{0}, t_{\alpha}^{1}$ for every $\alpha<\mu$. Then $\left\langle t_{\alpha}^{0}, t_{\alpha}^{1}\right.$ : $\alpha<\mu\rangle, u=\{1\}, v=\{0\}$ constitute an easy counterexample to the $(\mu, 2)$ entangledness of $\mathcal{I}$.

(6) Suppose $\mathcal{I}$ is $(\mu, \sigma)$-entangled and $|\mathcal{I}|=\lambda$. Take a sequence $\left\langle\mathcal{I}_{\varepsilon}\right.$ : $\varepsilon<\lambda\rangle$ of pairwise disjoint subsets of $\mathcal{I}$, each of power $\lambda$. This sequence witnesses $\operatorname{Ens}_{\sigma}(\lambda, \mu, \lambda)$ : suppose $u, v$ are disjoint subsets of $\lambda$ with $|u \cup v|<\sigma$ and let $t_{\alpha}^{\varepsilon} \in \mathcal{I}_{\varepsilon}$ for $\alpha<\mu$ and $\varepsilon \in u \cup v$ be pairwise distinct. Now apply "I is $(\mu, \sigma)$-entangled".

(7) Let $u, v, t_{\alpha}^{\varepsilon}$ (for $\varepsilon \in u \cup v, \alpha<\mu$ ) be as in Definition 1.1(3). Put

$$
\begin{gathered}
u^{\prime}=\{2 \varepsilon: \varepsilon \in u\} \cup\{2 \varepsilon+1: \varepsilon \in v\}, \quad v^{\prime}=\{2 \varepsilon: \varepsilon \in v\} \cup\{2 \varepsilon+1: \varepsilon \in u\}, \\
s_{\alpha}^{2 \varepsilon}=t_{2 \alpha}^{\varepsilon}, \quad s_{\alpha}^{2 \varepsilon+1}=t_{2 \alpha+1}^{\varepsilon} .
\end{gathered}
$$

Now we apply the 1.2(7) version of Definition 1.1(3) to $u^{\prime}, v^{\prime}$ and $\left\langle s_{\alpha}^{\varepsilon}: \varepsilon \in\right.$ $\left.u^{\prime} \cup v^{\prime}, \alpha<\mu\right\rangle$, and we get $\alpha^{\prime} \neq \beta^{\prime}$ as required there. If $\alpha^{\prime}<\beta^{\prime}$ then $\alpha=2 \alpha^{\prime}$ and $\beta=2 \beta^{\prime}$ are as required in 1.1(3). Otherwise $\alpha^{\prime}>\beta^{\prime}$ and then $\alpha=2 \beta^{\prime}+1$ and $\beta=2 \alpha^{\prime}+1$ are as required in 1.1(3).

(8) ( $\alpha$ ) Suppose $\lambda=\mu$ (and so $\operatorname{cf}(\mu) \geq \sigma)$ and let $\mathcal{I}$ be a $(\mu, \sigma)$-entangled linear order of size $\lambda$. Choose a family $\left\{A_{\varepsilon}: \varepsilon<\mu^{+}\right\} \subseteq[\mathcal{I}]^{\mu}$ such that $\left(\forall \varepsilon<\zeta<\mu^{+}\right)\left(\left|A_{\varepsilon} \cap A_{\zeta}\right|<\mu\right)$, and let $\mathcal{I}_{\varepsilon}=\mathcal{I}\left\lceil A_{\varepsilon}\right.$ (for $\left.\varepsilon<\mu^{+}\right)$. We claim that the sequence $\left\langle\mathcal{J}_{\varepsilon}: \varepsilon<\mu^{+}\right\rangle$witnesses $\operatorname{Ens}_{\sigma}\left(\lambda, \mu, \mu^{+}\right)$. Why? Clearly $\left|\mathcal{J}_{\varepsilon}\right|=\lambda=\mu$. Suppose that $u, v \subseteq \mu^{+}$are disjoint, $|u \cup v|<1+\sigma$ and for $\varepsilon \in u \cup v$ let $\left\langle t_{\alpha}^{\varepsilon}: \alpha<\mu\right\rangle \subseteq \mathcal{J}_{\varepsilon}$ be pairwise distinct. Since $\sigma \leq \operatorname{cf}(\mu)$ we find 
$\alpha(*)<\mu$ such that

$$
\left(\forall \varepsilon_{0}, \varepsilon_{1} \in u \cap v\right)\left(\forall \alpha_{0}, \alpha_{1}<\mu\right)\left(\varepsilon_{0} \neq \varepsilon_{1} \& \alpha_{0}, \alpha_{1}>\alpha(*) \Rightarrow t_{\alpha_{0}}^{\varepsilon_{0}} \neq t_{\alpha_{1}}^{\varepsilon_{1}}\right)
$$

(remember the choice of the $A_{\varepsilon}$ 's). Now apply the assumption that $\mathcal{I}$ is $(\mu, \sigma)$-entangled to the sequence

$$
\left\langle t_{\alpha}^{\varepsilon}: \varepsilon \in u \cup v, \alpha \in(\alpha(*), \mu)\right\rangle \subseteq \mathcal{I} .
$$

If $\lambda>\mu$ then we can choose a family $\left\{A_{\varepsilon}: \varepsilon<\mu^{+}\right\}$of pairwise disjoint sets from $[\mathcal{I}]^{\lambda}$ and proceed as above.

$(\beta),(\gamma)$ Similarly. $\mathbf{m}_{1.2}$

DEFINITION 1.3. Let $\mathcal{I}$ be a linear order.

(1) The interval Boolean algebra $\mathrm{BA}_{\text {inter }}(\mathcal{I})$ determined by $\mathcal{I}$ is the algebra of finite unions of closed-open intervals of $\mathcal{I}$ (including $[-\infty, x),[x, \infty$ ), $[-\infty, \infty))$

(2) For a regular cardinal $\sigma, \mathrm{BA}_{\text {inter }}^{\sigma}(\mathcal{I})$ is the closure of the family of subsets of $\mathcal{I}$ of the form $[-\infty, s)$ (for $s \in \mathcal{I}$ ) under complementation and taking unions and intersections of $<\sigma$ members $\left({ }^{1}\right)$.

Definition 1.4. Let $B$ be an infinite Boolean algebra.

(1) A set $Y \subseteq B$ is a pie if any two members of $Y$ are incomparable (in $B$; "pie" comes from "a set of pairwise incomparable elements").

(2) $\operatorname{inc}(B)=\sup \{|Y|: Y \subseteq B$ is a pie $\}$.

(3) $\operatorname{inc}^{+}(B)=\sup \left\{|Y|^{+}: Y \subseteq B\right.$ is a pie $\}$.

(4) The algebra $B$ is $\mu$-narrow if there is no pie of cardinality $\geq \mu$.

(5) Length $(B)=\sup \{|Y|: Y \subseteq B$ is a chain $\}$,

Length $^{+}(B)=\sup \left\{|Y|^{+}: Y \subseteq B\right.$ is a chain $\}$.

Proposition 1.5. Suppose that $\mathcal{I}$ is a linear order and that the regular cardinals $\aleph_{0} \leq \sigma<\mu$ satisfy $(\forall \theta<\mu)\left[\theta^{<\sigma}<\mu\right]$. Then the following conditions are equivalent:

(a) The order $\mathcal{I}$ is $(\mu, \sigma)$-entangled.

(b) If $\varepsilon(*)<\sigma$, and $u, v \subseteq \varepsilon(*)$ are disjoint and $t_{\alpha}^{\varepsilon} \in \mathcal{I}$ (for $\varepsilon<\varepsilon(*)$ and $\alpha<\mu$ ) then for some $\alpha<\beta<\mu$ we have

$$
\varepsilon \in u \Rightarrow t_{\alpha}^{\varepsilon} \leq_{\mathcal{I}} t_{\beta}^{\varepsilon} \quad \text { and } \quad \varepsilon \in v \Rightarrow t_{\alpha}^{\varepsilon} \geq_{\mathcal{I}} t_{\beta}^{\varepsilon} .
$$

(Note: if the $t_{\alpha}^{\varepsilon}$ are pairwise distinct then the inequalities are in fact strict; as in the proof of 1.2(7), changing the demand $\alpha<\beta$ to $\alpha \neq \beta$ does not matter.)

(c) The algebra $\mathrm{BA}_{\text {inter }}^{\sigma}(\mathcal{I})$ is $\mu$-narrow.

$\left({ }^{1}\right)$ Equivalently, the Boolean algebra $\sigma$-generated by $\left\{x_{t}: t \in \mathcal{I}\right\}$ freely except $x_{s} \leq x_{t}$ when $\mathcal{I} \models s<t$. 
Proof. (a) $\Rightarrow(\mathrm{c})$. By 1.2(5) the order $\mathcal{I}$ has density $<\mu$. Let $\left\langle A_{\alpha}: \alpha<\right.$ $\mu\rangle$ be a sequence of distinct elements of the algebra $\mathrm{BA}_{\text {inter }}^{\sigma}(\mathcal{I})$. We know that for each $\alpha$ there are: an ordinal $\varepsilon_{\alpha}<\sigma$, a Boolean term $\tau_{\alpha}$ (with all unions and intersections of size $<\sigma$ and $\varepsilon_{\alpha}$ free variables) and a sequence $\left\langle t_{\alpha}^{\varepsilon}: \varepsilon\left\langle\varepsilon_{\alpha}\right\rangle \subseteq \mathcal{I}\right.$ such that $A_{\alpha}=\tau_{\alpha}\left(\ldots, t_{\alpha}^{\varepsilon}, \ldots\right)_{\varepsilon<\varepsilon_{\alpha}}$. By 1.2(4), without loss of generality for some $\varepsilon(*)$ and pairwise disjoint intervals $\left[a_{\varepsilon}, b_{\varepsilon}\right]$ we have $\varepsilon_{\alpha}=\varepsilon(*)$ and for each $\varepsilon<\varepsilon(*)$ either $(\forall \alpha<\mu)\left(a_{\varepsilon}<t_{\alpha}^{\varepsilon}<b_{\varepsilon}\right)$ or $(\forall \alpha<\mu)\left(t_{\alpha}^{\varepsilon}=a_{\varepsilon}\right)$. Since $\mu=\operatorname{cf}(\mu)>\aleph_{0}$ and $(\forall \theta<\mu)\left(\theta^{|\varepsilon(*)|}<\mu\right)$ we may apply the $\Delta$-lemma to the family $\left\{x_{\alpha}: \alpha<\mu\right\}$, where $x_{\alpha}:=\left\{t_{\alpha}^{\varepsilon}: \varepsilon<\varepsilon(*)\right\}$. Consequently, we may assume that $\left\{x_{\alpha}: \alpha<\mu\right\}$ forms a $\Delta$-system with the kernel $x$ (i.e. $\alpha<\beta<\mu \Rightarrow x_{\alpha} \cap x_{\beta}=x$ ). Note that if $t_{\alpha}^{\varepsilon} \in x$ for some $\alpha<\mu$ then $(\forall \alpha<\beta<\mu)\left(t_{\alpha}^{\varepsilon}=t_{\beta}^{\varepsilon}\right)$ and if $t_{\alpha}^{\varepsilon} \notin x$ for some $\alpha<\mu$ then $(\forall \alpha<\beta<\mu)\left(t_{\alpha}^{\varepsilon} \neq t_{\beta}^{\varepsilon}\right)$. Thus for each $\varepsilon<\varepsilon(*)$ either $t_{\alpha}^{\varepsilon}($ for $\alpha<\mu$ ) are pairwise distinct or they are pairwise equal. Since $\mu=\operatorname{cf}(\mu)>\sigma$ and $\theta^{<\sigma}<\mu$ for $\theta<\mu$, without loss of generality $\tau_{\alpha}=\tau$. Let

$$
w=\left\{\varepsilon<\varepsilon(*):\left\langle t_{\alpha}^{\varepsilon}: \alpha<\mu\right\rangle \text { are pairwise distinct }\right\} .
$$

Then for some disjoint sets $v, u \subseteq w$ and a set $A \subseteq \mathcal{I} \backslash \bigcup_{\varepsilon \in u \cup v}\left[a_{\varepsilon}, b_{\varepsilon}\right]$ we have

$$
A_{\alpha}=A \cup \bigcup_{\varepsilon \in u \cup v} \tau^{\varepsilon}\left(a_{\varepsilon}, t_{\alpha}^{\varepsilon}, b_{\varepsilon}\right)
$$

where we let

$$
\tau^{\varepsilon}(x, y, z)= \begin{cases}{[x, y)} & \text { if } \varepsilon \in u \\ {[y, z)} & \text { if } \varepsilon \in v\end{cases}
$$

Since $\mathcal{I}$ is $(\mu, \sigma)$-entangled, we can find $\alpha<\beta$ such that

$$
(\forall \varepsilon \in u \cup v)\left(t_{\alpha}^{\varepsilon}<t_{\beta}^{\varepsilon} \Leftrightarrow \varepsilon \in u\right) .
$$

Clearly this implies that $A_{\alpha} \subseteq A_{\beta}$, so we are done.

(c) $\Rightarrow$ (a). First we note that the linear order $\mathcal{I}$ has density $<\mu$. [Why? Clearly $\mathcal{I}$ has no well ordered subset of power $\mu$ nor an inverse well ordered subset of power $\mu$. Assume $\mathcal{I}$ has density $\geq \mu$. First we show that there are disjoint closed-open intervals $\mathcal{I}_{0}, \mathcal{I}_{1}$ of $\mathcal{I}$ with density $\geq \mu$. To prove the existence of $\mathcal{I}_{0}, \mathcal{I}_{1}$ define the relation $E$ on $\mathcal{I}$ by

$$
\begin{array}{cl}
a E b \quad \text { if and only if } & a=b \text { or }[a<b \text { and density }([a, b))<\mu] \text { or } \\
& {[a>b \text { and density }([b, a))<\mu] .}
\end{array}
$$

Clearly $E$ is an equivalence relation and its equivalence classes are convex. Moreover, the density of each $E$-equivalence class is less than $\mu$ (as there is no monotonic sequence of length $\mu$ of members of $\mathcal{I}$ ). Consequently, we find $a, b \in \mathcal{I}$ such that $a<b$ and $\neg a E b$. Next we can find $c, d \in(a, b)$ with $c<d$ such that neither $a E c$ nor $d E b$. Thus we may put $\mathcal{I}_{0}=[a, c)$ and $\mathcal{I}_{1}=[d, b)$. Now for each $\mathcal{I}_{m}$ we choose by induction on $\beta<\mu$ elements 
$a_{\beta}^{m}<b_{\beta}^{m}$ from $\mathcal{I}_{m}$ such that $\left[a_{\beta}^{m}, b_{\beta}^{m}\right]$ is disjoint from $\left\{a_{\alpha}^{m}, b_{\alpha}^{m}: \alpha<\beta\right\}$. So $\alpha<\beta \Rightarrow\left[a_{\alpha}^{m}, b_{\alpha}^{m}\right) \nsubseteq\left[a_{\beta}^{m}, b_{\beta}^{m}\right)$. Now, $\left\langle\left[a_{\beta}^{0}, b_{\beta}^{0}\right) \cup\left(\mathcal{I}_{1} \backslash\left[a_{\beta}^{1}, b_{\beta}^{1}\right)\right): \beta<\mu\right\rangle$ shows that the algebra $\mathrm{BA}_{\text {inter }}^{\sigma}(\mathcal{I})$ is not $\mu$-narrow, a contradiction].

By 1.2(7) it is enough to prove that if $\varepsilon(*)<\sigma$ and $t_{\alpha}^{\varepsilon} \in \mathcal{I}$ are distinct for $\alpha<\mu, \varepsilon<\varepsilon(*)$ and $u, v$ are disjoint subsets of $\varepsilon(*)$ then we can find $\alpha \neq \beta$ such that

$$
\varepsilon \in u \Rightarrow t_{\alpha}^{\varepsilon}<t_{\beta}^{\varepsilon} \quad \text { and } \quad \varepsilon \in v \Rightarrow t_{\alpha}^{\varepsilon}>t_{\beta}^{\varepsilon} .
$$

By 1.2(4), without loss of generality for some pairwise disjoint intervals $\left[a_{\varepsilon}, b_{\varepsilon}\right]$ of $\mathcal{I}$, we have $t_{\alpha}^{\varepsilon} \in\left(a_{\varepsilon}, b_{\varepsilon}\right)$. Let $x_{\alpha}:=x_{\alpha}^{1} \cup x_{\alpha}^{2}$, where

$$
x_{\alpha}^{1}:=\bigcup\left\{\left[a_{\varepsilon}, t_{\alpha}^{\varepsilon}\right): \varepsilon \in u\right\}, \quad x_{\alpha}^{2}:=\bigcup\left\{\left[t_{\alpha}^{\varepsilon}, b_{\varepsilon}\right): \varepsilon \in v\right\} .
$$

So $x_{\alpha} \in \mathrm{BA}_{\text {inter }}^{\sigma}(\mathcal{I})$ for $\alpha<\mu$. The algebra $\mathrm{BA}_{\text {inter }}^{\sigma}(\mathcal{I})$ is $\mu$-narrow, so for some $\alpha \neq \beta(<\mu)$ we have $x_{\alpha} \subseteq x_{\beta}$. Then for each $\varepsilon$,

$$
\varepsilon \in u \Rightarrow \mathcal{I} \models t_{\alpha}^{\varepsilon} \leq t_{\beta}^{\varepsilon} \quad \text { and } \quad \varepsilon \in v \Rightarrow \mathcal{I} \models t_{\alpha}^{\varepsilon} \geq t_{\beta}^{\varepsilon} .
$$

This is as required.

$(a) \Rightarrow(b)$. This is included in the proof of $(a) \Rightarrow(c)$.

(b) $\Rightarrow(\mathrm{a})$. Trivial. $\mathbf{a}_{1.4}$

Proposition 1.6. Let $B$ be a Boolean algebra.

(1) If $\mathrm{inc}^{+}(B)$ is a successor cardinal then $[\operatorname{inc}(B)]^{+}=\operatorname{inc}^{+}(B)$.

(2) If $\operatorname{inc}^{+}(B)$ is a limit cardinal then $\operatorname{inc}(B)=\operatorname{inc}^{+}(B)$.

(3) $B$ is $\mu$-narrow if and only if $\operatorname{inc}^{+}(B) \leq \mu$.

(4) If $B$ is $\mu$-narrow then so is every homomorphic image of $B$.

(5) If $D$ is a filter on $\sigma$ and the product algebra $B^{\sigma}$ is $\mu$-narrow then the algebra $B^{\sigma} / D$ is $\mu$-narrow. 1.6

Conclusion 1.7. Assume $\lambda^{*} \geq \mu, \lambda \geq \mu=\operatorname{cf}(\mu)>\kappa \geq \sigma=\operatorname{cf}(\sigma) \geq \aleph_{0}$ and $(\forall \theta<\mu)\left[\theta^{<\sigma}<\mu\right]$.

(1) Then $(A)_{\lambda^{*}, \lambda, \mu, \sigma, \kappa} \Rightarrow(B)_{\lambda^{*}, \lambda, \mu, \sigma, \kappa}$, using $B_{j}=\mathrm{BA}_{\text {inter }}\left(\mathcal{I}_{j}+\mathcal{J}_{j}\right)$, where

$(A)_{\lambda^{*}, \lambda, \mu, \sigma, \kappa} \quad$ there are linear orders $\mathcal{I}_{j}, \mathcal{J}_{j}($ for $j \leq \kappa)$ of cardinality $\lambda$ such that each $\mathcal{I}_{j}+\mathcal{J}_{j}$ is $(\mu, \sigma)$-entangled and for any uniform ultrafilter $D$ on $\kappa$ the linear orders $\prod_{j<\kappa} \mathcal{I}_{j} / D$ and $\prod_{j<\kappa} \mathcal{J}_{j} / D$ have isomorphic subsets of cardinality $\lambda^{*}$;

$(B)_{\lambda^{*}, \lambda, \mu, \sigma, \kappa} \quad$ there are interval Boolean algebras $B_{j}($ for $j<\kappa)$ which are $\mu$-narrow and of cardinality $\lambda$ such that for any uniform ultrafilter $D$ on $\kappa$ the algebra $B=\prod_{i<\kappa} B_{i} / D$ is not $\lambda^{*}$ narrow.

(2) Also $(A)_{\lambda^{*}, \lambda, \mu, \sigma, \kappa}^{+} \Rightarrow(B)_{\lambda^{*}, \lambda, \mu, \sigma, \kappa}^{+}\left(\right.$using $\left.B=\mathrm{BA}_{\text {inter }}(\mathcal{I}+\mathcal{J})\right)$, where 
$(A)_{\lambda^{*}, \lambda, \mu, \sigma, \kappa}^{+} \quad$ there are linear orders $\mathcal{I}, \mathcal{J}$ of cardinality $\lambda$ such that $\mathcal{I}+\mathcal{J}$ is $(\mu, \sigma)$-entangled and for any uniform ultrafilter $D$ on $\kappa$ the linear orders $\mathcal{I}^{\kappa} / D$ and $\mathcal{J}^{\kappa} / D$ have isomorphic subsets of cardinality $\lambda^{*}$;

$(B)_{\lambda^{*}, \lambda, \mu, \sigma, \kappa}^{+} \quad$ there is a $\mu$-narrow interval Boolean algebra $B$ of cardinality $\lambda$ such that $\lambda^{*}<$ inc $^{+}\left[B^{\kappa} / D\right]$ for any uniform ultrafilter $D$ on $\kappa$ (i.e. the algebra is not $\lambda^{*}$-narrow).

(3) We can replace "uniform ultrafilter D" by "regular ultrafilter D" or fix a filter $D$ on $\kappa$.

P r o of. Just note that if $B$ is a Boolean algebra, $\mathcal{I}, \mathcal{J}$ are linear orders, $a_{t} \in B$ for $t \in \mathcal{I}+\mathcal{J}$ are such that $t<s \Rightarrow a_{t}<_{B} a_{s}$ and $f$ is an (order) isomorphism from $\mathcal{I}$ to $\mathcal{J}$ then $\left\{a_{f(t)}-a_{t}: t \in \mathcal{I}\right\}$ is a pie of $B . \mathbf{m}_{1.7}$

Conclusion 1.8. Assume that $\sigma<\mu^{\kappa}=\mu<\lambda$ and there is a $(\mu, \sigma)$ entangled linear order $\mathcal{I}+\mathcal{J}$ such that for each uniform ultrafilter $D$ on $\kappa$ the linear orderings $\mathcal{I}^{\kappa} / D$ and $\mathcal{J}^{\kappa} / D$ contain isomorphic subsets of cardinality $\lambda>\mu$. Then

$$
\operatorname{inc}^{+}\left(\mathrm{BA}_{\text {inter }}(\mathcal{I}+\mathcal{J})\right) \leq \mu \quad \text { and } \quad \operatorname{inc}\left(\left(\mathrm{BA}_{\text {inter }}(\mathcal{I}+\mathcal{J})\right)^{\kappa} / D\right) \geq \lambda
$$

and even

$$
\operatorname{inc}^{+}\left(\left(\mathrm{BA}_{\text {inter }}(\mathcal{I}+\mathcal{J})\right)^{\kappa} / D\right)>\lambda
$$

(so inc $\left.\left(\left(\mathrm{BA}_{\text {inter }}(\mathcal{I}+\mathcal{J})\right)^{\kappa} / D\right)>\operatorname{inc}\left(\mathrm{BA}_{\text {inter }}(\mathcal{I}+\mathcal{J})\right)^{\kappa} / D\right)$.

Re mark. See an example in 3.2(3).

Definition 1.9. We say that a linear order $\mathcal{I}$ has exact $(\lambda, \mu, \kappa)$-density if for every $\mathcal{J} \subseteq \mathcal{I}$ of cardinality $\geq \lambda$ we have $\operatorname{density}(\mathcal{J}) \in[\kappa, \mu)$.

If $\mu=\kappa^{+}$we may omit $\mu$; if $\lambda=|\mathcal{I}|$ we may omit it. We may also say $\mathcal{I}$ has exact density $(\lambda, \mu, \kappa)$ or $(\lambda, \mu, \kappa)$ is an exact density of $\mathcal{I}$ (and replace $(\lambda, \mu, \kappa)$ by $(\lambda, \mu)$ or $(\mu, \kappa)$ or $\kappa)$.

DeFinition 1.10. (1) A linear order $\mathcal{I}$ is positively $\sigma$-entangled if for each $\varepsilon(*)<1+\sigma, u \in\{\emptyset, \varepsilon(*)\}$ and an indexed set $\left\{t_{\varepsilon}^{\alpha}: \alpha<|\mathcal{I}|, \varepsilon<\varepsilon(*)\right\} \subseteq \mathcal{I}$ such that

$$
(\forall \alpha<\beta<|\mathcal{I}|)(\forall \varepsilon<\varepsilon(*))\left(t_{\varepsilon}^{\alpha} \neq t_{\varepsilon}^{\beta}\right)
$$

there exist $\alpha<\beta<|\mathcal{I}|$ such that $(\forall \varepsilon<\varepsilon(*))\left(\varepsilon \in u \Leftrightarrow t_{\varepsilon}^{\alpha}<t_{\varepsilon}^{\beta}\right)$.

(2) Similarly we define when $\overline{\mathcal{I}}=\left\langle\mathcal{I}_{i}: i<i^{*}\right\rangle$ is positively $\sigma$-entangled and $\operatorname{PosEns}_{\sigma}(\lambda, \mu, \kappa), \operatorname{PosEns}_{\sigma}(\lambda, \kappa)$.

For more on entangledness in ultraproducts see Section 6.

2. Constructions for $\lambda=\lambda^{<\lambda}$. In this section we will build entangled linear orders from instances of GCH. Our main tool here is the construction principle presented in [HLSh 162] and developed in [Sh 405]. The main point 
of the principle is that for standard $\lambda^{+}$-semiuniform partial orders (see 2.1 below) there are "sufficiently generic" filters $G$, provided $\nabla_{\lambda}$ holds (actually, a weaker assumption suffices). For the precise definition of "sufficiently generic" we refer the reader to [Sh 405, Appendix] (compare also [HLSh 162, $\S 1])$. Here we recall the definition of standard $\lambda^{+}$-semiuniform partial orders, as it lists the conditions we will have to check later.

Definition 2.1. Let $\lambda$ be a regular cardinal.

(1) A set $u \subseteq \lambda^{+}$is closed if $0 \in u$ and $\delta=\sup (\delta \cap u) \Rightarrow \delta \in u$.

(2) Let $(\mathcal{P}, \leq)$ be a partial order such that

$$
\mathcal{P} \subseteq \lambda \times\left\{u \subseteq \lambda^{+}:|u|<\lambda^{+} \& u \text { is closed }\right\} .
$$

If $p=(\alpha, u) \in \mathcal{P}$ then we write $\operatorname{dom}(p)=u$. For an ordinal $\beta<\lambda^{+}$ we let $\mathcal{P}_{\beta}=\{p \in \mathcal{P}: \operatorname{dom}(p) \subseteq \beta\}$. We say that $(\mathcal{P}, \leq)$ is a standard $\lambda^{+}$-semiuniform partial order if the following conditions are satisfied:

(a) If $p \leq q$ then $\operatorname{dom}(p) \subseteq \operatorname{dom}(q)$.

(b) If $p \in \mathcal{P}, \alpha<\lambda^{+}$is either a successor ordinal or $\operatorname{cf}(\alpha)=\lambda$ then there exists $q \in \mathcal{P}$ such that $q \leq p$ and $\operatorname{dom}(q)=\operatorname{dom}(p) \cap$ $\alpha$; moreover, there is a unique maximal such $q$ which will be denoted by $p\lceil\alpha$.

(c) If $p=(\alpha, u) \in \mathcal{P}, h: u \stackrel{1-1}{\longrightarrow} v \subseteq \lambda^{+}$is an order isomorphism onto $v$ such that $(\forall \alpha \in u)(\operatorname{cf}(\alpha)=\lambda \Leftrightarrow \operatorname{cf}(h(\alpha))=\lambda)$ and $v$ is closed then $h[p]:=(\alpha, v) \in \mathcal{P}$; moreover, $q \leq p$ implies $h[q] \leq h[p]$.

(d) If $p, q \in \mathcal{P}, \alpha<\lambda^{+}$is either a successor ordinal or $\operatorname{cf}(\alpha)=\lambda$ and $p\left\lceil\alpha \leq q \in \mathcal{P}_{\alpha}\right.$ then there is $r \in \mathcal{P}$ such that $p, q \leq r$.

(e) If $\left\langle p_{i}: i<\delta\right\rangle \subseteq \mathcal{P}$ is an increasing sequence and $\delta<\lambda$ then there is $q \in \mathcal{P}$ such that

$$
\operatorname{dom}(q)=\operatorname{cl}\left(\bigcup_{i<\delta} \operatorname{dom}\left(p_{i}\right)\right) \text { and } \quad(\forall i<\delta)\left(p_{i} \leq q\right) .
$$

(f) Suppose that $\left\langle p_{i}: i<\delta\right\rangle \subseteq \mathcal{P}_{\beta+1}$ is increasing, $\delta<\lambda$ and $\beta<\lambda^{+}$ has cofinality $\lambda$. Assume that $q \in \mathcal{P}_{\beta}$ is such that $(\forall i<\delta)$ $\left(p_{i} \uparrow \beta \leq q\right)$. Then the family $\left\{p_{i}: i<\delta\right\} \cup\{q\}$ has an upper bound $r$ such that $q \leq r\lceil\beta$.

(g) Assume that $\left\langle\beta_{i}: i<\delta\right\rangle \subseteq \lambda^{+}$is strictly increasing, each $\beta_{i}$ is either a successor or has cofinality $\lambda$, and $\delta<\lambda$ is a limit ordinal. Suppose that $q \in \mathcal{P}$ and $(\forall i<\delta)\left(q\left\lceil\beta_{i} \leq p_{i} \in \mathcal{P}_{\beta_{i}}\right)\right.$, and $\left\langle p_{i}: i<\delta\right\rangle \subseteq \mathcal{P}$ is increasing. Then the family $\left\{p_{i}: i<\delta\right\} \cup\{q\}$ has an upper bound $r \in \mathcal{P}$ such that $(\forall i<\delta)\left(p_{i} \leq r\left\lceil\beta_{i}\right)\right.$.

(h) Suppose that $\delta_{1}, \delta_{2}<\lambda$ are limit ordinals and $\left\langle\beta_{j}: j<\delta_{2}\right\rangle \subseteq \lambda^{+}$ is a strictly increasing sequence of ordinals, each $\beta_{j}$ either a 
successor or of cofinality $\lambda$. Let

$$
\left\langle p_{i, j}:(i, j) \in\left(\delta_{1}+1\right) \times\left(\delta_{2}+1\right) \backslash\left\{\left(\delta_{1}, \delta_{2}\right)\right\}\right\rangle \subseteq \mathcal{P}
$$

be such that

$$
p_{i, j} \in \mathcal{P}_{\beta_{j}}, \quad i \leq i^{\prime} \Rightarrow p_{i, j} \leq p_{i^{\prime}, j}, \quad j \leq j^{\prime} \Rightarrow p_{i, j} \leq p_{i, j^{\prime}} \uparrow \beta_{j} .
$$

Then the family $\left\{p_{i, j}:(i, j) \in\left(\delta_{1}+1\right) \times\left(\delta_{2}+1\right) \backslash\left\{\left(\delta_{1}, \delta_{2}\right)\right\}\right\}$ has an upper bound $r \in \mathcal{P}$ such that $\left(\forall j<\delta_{2}\right)\left(r\left\lceil\beta_{j}=p_{\delta_{1}, j}\right)\right.$.

Notation 2.2. Let $\lambda, \mu$ be cardinals and $T$ be a tree.

(1) For an ordinal $\alpha$, the $\alpha$ th level of the tree $T$ is denoted by $T_{\alpha}$; for $x \in T, \operatorname{lev}(x)$ is the unique $\alpha$ such that $x \in T_{\alpha}$.

(2) We say that the tree $T$ is normal if for each $y, z \in T$ we have: if $(\forall x \in T)\left(x<_{T} y \equiv x<_{T} z\right)$ and $\operatorname{lev}(y)=\operatorname{lev}(z)$ is a limit ordinal then $y=z$. Usually we assume that $T$ is normal.

(3) We say that the tree $T$ is $\lambda^{+}$-Aronszajn if it has $\lambda^{+}$levels, each level is of size $\leq \lambda$, there is no $\lambda^{+}$-branch in $T, T$ is normal, and

$$
y \in T, \operatorname{lev}(y)<\beta<\lambda^{+} \Rightarrow(\exists z \in T)\left[y<_{T} z \& \operatorname{lev}(z)=\beta\right] .
$$

(4) For ordinals $\zeta$ and $\alpha$ let $T_{\alpha}^{[\zeta]}$ be the set of all sequences of length $\zeta$ with no repetition from $T_{\alpha}$. We let $T^{[\zeta]}=\bigcup_{\alpha} T_{\alpha}^{[\zeta]}$, but we may identify $T^{[1]}$ and $T$ (and similarly for $T^{\langle 1\rangle}$ below).

(5) For a sequence $\bar{x} \in T^{[\zeta]}$, let $\operatorname{lev}(\bar{x})$ be the unique $\alpha$ such that $\bar{x} \in T_{\alpha}^{[\zeta]}$.

(6) For $\bar{x}, \bar{y} \in T^{[\zeta]}$, let $\bar{x}<\bar{y}$ mean $(\forall \varepsilon<\zeta)\left(x_{\varepsilon}<_{T} y_{\varepsilon}\right)$; similarly for $\bar{x} \leq \bar{y}$.

(7) Let $\bar{x} \in T_{\alpha}^{[\zeta]}$. We define $T_{\bar{x}}^{[\zeta]}:=\left\{\bar{y} \in T^{[\zeta]}: \bar{x}<_{T} \bar{y}\right\}$.

(8) $T^{\langle\zeta\rangle}=\bigcup\left\{T_{\alpha}^{[\zeta]}\right.$ : either $\alpha$ is a successor ordinal or $\left.\operatorname{cf}(\alpha)=\lambda\right\}$ and $T_{\bar{x}}^{\langle\zeta\rangle}=T_{\bar{x}}^{[\zeta]} \cap T^{\langle\zeta\rangle}$.

(9) For $x, y \in T$ let $x \wedge y$ be their maximal lower bound (in $T$, exists when $T$ is normal).

(10) For $x \in T$ and an ordinal $\alpha \leq \operatorname{lev}(x)$ let $x\left\lceil\alpha\right.$ be the unique $y \leq_{T} x$ such that $\operatorname{lev}(y)=\alpha$.

(11) For $\bar{x}=\left\langle x_{\varepsilon}: \varepsilon<\zeta\right\rangle \in T^{[\zeta]}$ and an ordinal $\alpha \leq \operatorname{lev}(\bar{x})$ let $\bar{x}\lceil\alpha=$ $\left\langle x_{\varepsilon} \mid \alpha: \varepsilon<\zeta\right\rangle$.

(12) Let $H_{\mu}^{1}$ be the family of all functions $h$ with domains included in $\bigcup\left\{\zeta\left(\mu^{+} \times \mu^{+}\right): \zeta<\mu\right\}$ and such that for $\zeta<\mu$ and $\bar{x} \in \zeta\left(\mu^{+} \times \mu^{+}\right)$we have: $h(\bar{x}) \subseteq{ }^{\zeta}\left(\mu^{+}\right)$(if defined, and then) there are $\mu^{+}$members of $h(\bar{x})$ with pairwise disjoint ranges.

If $h \in H_{\mu}^{1}$ is a partial function, $\zeta<\mu, \bar{x} \in{ }^{\zeta}\left(\mu^{+}\right)$and $h(\bar{x})$ is not defined then $h(\bar{x})$ will mean ${ }^{\zeta}\left(\mu^{+}\right)$. 
We use mainly $h \in H_{\mu, *}^{1}$ where

$$
H_{\mu, *}^{1}=\bigcup_{\zeta<\mu} H_{\mu, \zeta}^{1}, \quad H_{\mu, \zeta}^{1}=\left\{h \in H_{\mu}^{1}: \operatorname{dom}(h)={ }^{\zeta}\left(\mu^{+} \times \mu^{+}\right)\right\} .
$$

(13) Let $H_{\mu}^{0}$ be the set of all $h$ from $H_{\mu}^{1}$ such that the value of $h\left(\left\langle\left(\alpha_{\varepsilon}^{0}, \alpha_{\varepsilon}^{1}\right)\right.\right.$ : $\varepsilon<\zeta\rangle$ ) does not depend on $\left\langle\alpha_{\varepsilon}^{0}: \varepsilon<\zeta\right\rangle$ (so we may write $h\left(\left\langle\alpha_{\varepsilon}^{1}: \varepsilon<\zeta\right\rangle\right)$ ).

(14) Let $H_{\mu}^{3}$ be the family of all functions $h$ with domain $\mu$ such that $h(\zeta)$ is a subset of $\zeta\left(\left(\mu^{+}\right)^{3}\right)$ with the following property:

(छ) for each $\left\langle\alpha_{\varepsilon}^{0}: \varepsilon\langle\zeta\rangle \subseteq \mu^{+}\right.$and every $\beta<\mu^{+}$there is $\left\langle\alpha_{\varepsilon}^{1}: \varepsilon<\zeta\right\rangle \subseteq$ $\left(\beta, \mu^{+}\right)$with

$$
\left(\forall \beta^{\prime}<\mu^{+}\right)\left(\exists\left\langle\alpha_{\varepsilon}^{2}: \varepsilon<\zeta\right\rangle \subseteq\left(\beta^{\prime}, \mu^{+}\right)\right)\left(\left\langle\left(\alpha_{\varepsilon}^{0}, \alpha_{\varepsilon}^{1}, \alpha_{\varepsilon}^{2}\right): \varepsilon<\zeta\right\rangle \in h(\zeta)\right) .
$$

(15) $H_{\mu}^{2}$ is the collection of those $h \in H_{\mu}^{3}$ such that the truth value of " $\left\langle\left(\alpha_{\varepsilon}^{0}, \alpha_{\varepsilon}^{1}, \alpha_{\varepsilon}^{2}\right): \varepsilon<\zeta\right\rangle \in h(\zeta)$ " does not depend on $\left\langle\alpha_{\varepsilon}^{0}: \varepsilon \in \zeta\right\rangle$ (so we may write $\left.\left\langle\left(\alpha_{\varepsilon}^{1}, \alpha_{\varepsilon}^{2}\right): \varepsilon<\mu\right\rangle \in h(\zeta)\right)$.

Theorem 2.3. Suppose $\lambda=\mu^{+}=2^{\mu}$ and $\diamond_{\lambda}$ (the second follows e.g. if $\mu \geq \beth_{\omega}$; see $[$ Sh 460, 3.5(1)]).

(1) There exists a dense linear order $\mathcal{I}$ of cardinality $\lambda^{+}$and density $\lambda^{+}$ (really exact density $\lambda^{+}$, see 1.9 ) such that:

$(*)_{1} \quad \mathcal{I}$ is hereditarily of cellularity $\lambda^{+}$, i.e. every interval in $\mathcal{I}$ contains $\lambda^{+}$pairwise disjoint subintervals, and

$(*)_{2} \quad \mathcal{I}$ is $\mu$-locally entangled, i.e. if $\kappa<\mu$ and $\left(a_{i}, b_{i}\right)_{\mathcal{I}}($ for $i<\kappa)$ are pairwise disjoint intervals then the sequence $\left\langle\mathcal{I}\left\lceil\left(a_{i}, b_{i}\right): i<\kappa\right)\right\rangle$ is $\kappa^{+}$-entangled $\left({ }^{2}\right)$.

(2) Let $H_{\mu}^{1, *} \subseteq H_{\mu}^{1}$ and $H_{\mu}^{3, *} \subseteq H_{\mu}^{3}$ have cardinality $\leq \lambda$. There is a $\lambda^{+}$-Aronszajn tree $T \subseteq{ }^{\lambda^{+}}>\lambda$ in which each node has $\lambda$ immediate successors and there are two functions $c, \bar{d}$ such that:

(a) $c$ is a function from $T$ to $\lambda$,

(b) for every $\bar{x} \in T^{[\mu]}$ and a function $h \in H_{\mu}^{1, *} \cup H_{\mu}^{3, *}$ we have $d_{\bar{x}, h}: T_{\bar{x}}^{[\mu]} \rightarrow \lambda$ such that if $\bar{y}, \bar{z} \in T_{\bar{x}}^{\langle\mu\rangle}$ are distinct and $d_{\bar{x}, h}(\bar{y})=$ $d_{\bar{x}, h}(\bar{z})$ then for some $\bar{t} \in T_{\bar{x}}^{[\mu]}$ we have

( $\alpha) t_{i}=y_{i} \wedge z_{i}$, and the values of $\operatorname{lev}\left(t_{i}\right)$ do not depend on $i$,

$(\beta) \operatorname{lev}(\bar{t})<\operatorname{lev}(\bar{z}), \operatorname{lev}(\bar{t})<\operatorname{lev}(\bar{y})$,

$(\gamma)$ if $d_{\bar{x}, h}(\bar{y})<d_{\bar{x}, h}(\bar{t})$ then $(\forall \varepsilon<\mu)\left(\exists^{\mu} i<\mu\right)\left(c\left(t_{i}\right)=\varepsilon\right)$,

$(\delta)$ if $\zeta<\mu$ and $h \in H_{\mu}^{1, *}$ then for $\mu$ ordinals $i<\mu$ divisible by $\zeta$ we have either

$\left({ }^{2}\right)$ Note: for $\mu \in(2, \lambda), \lambda=\operatorname{cf}(\lambda)$ such that $(\forall \alpha<\lambda)\left(|\alpha|^{<\mu}<\lambda\right)$ and a linear order $\mathcal{I}$ of cardinality $\lambda$ we have: $\mathcal{I}$ is $\mu$-entangled if and only if $\mathcal{I}$ is $\mu$-locally entangled of density $<\lambda$. 
(i) $\left\langle z_{i+\varepsilon}(\operatorname{lev}(\bar{t})): \varepsilon<\zeta\right\rangle \in h\left(\left\langle c\left(t_{i+\varepsilon}\right), y_{i+\varepsilon}(\operatorname{lev}(\bar{t})): \varepsilon<\zeta\right\rangle\right)$, or

(ii) $\left\langle y_{i+\varepsilon}(\operatorname{lev}(\bar{t})): \varepsilon<\zeta\right\rangle \in h\left(\left\langle c\left(t_{i+\varepsilon}\right), z_{i+\varepsilon}(\operatorname{lev}(\bar{t})): \varepsilon<\zeta\right\rangle\right)$,

$(\varepsilon)$ if $\zeta<\mu$ and $h \in H_{\mu}^{3, *}$ and $d_{\bar{x}, h}(\bar{y})<d_{\bar{x}, h}(\bar{t})$ then for $\mu$ ordinals $i<\mu$ divisible by $\zeta$ we have either

(i) $\left\langle\left(c\left(t_{i+\varepsilon}\right), y_{i+\varepsilon}(\operatorname{lev}(\bar{t})), z_{i+\varepsilon}(\operatorname{lev}(\bar{t}))\right): \varepsilon<\zeta\right\rangle \in h(\zeta)$, or

(ii) $\left\langle\left(c\left(t_{i+\varepsilon}\right), z_{i+\varepsilon}(\operatorname{lev}(\bar{t})), y_{i+\varepsilon}(\operatorname{lev}(\bar{t}))\right): \varepsilon<\zeta\right\rangle \in h(\zeta)$.

Explanation. Some points in 2.3(2) may look unnatural.

(1) Why $\bar{y}, \bar{z} \in T_{\bar{x}}^{\langle\mu\rangle}$ and not $\operatorname{dom}\left(d_{\bar{x}, h}\right)$ ? As in proving amalgamation we should compare $\left\langle x_{i}^{\beta}: i<\mu\right\rangle$ and $\left\langle x_{i}^{\alpha}: i<\mu\right\rangle$; necessary when $\alpha=\sup \left(w^{q}\right)$. However, working a little bit harder we may waive this.

(2) Why e.g. in clause $(\mathrm{b})(\varepsilon)$ we demand $d_{\bar{x}, h}(\bar{y})<d_{\bar{x}, h}(\bar{t})$ ? Otherwise we will not be able to prove the density of

$$
\mathcal{D}_{\bar{x}, h, \bar{y}}:=\left\{p \in \mathcal{A P}: \bar{y} \in \operatorname{dom}\left(d_{\bar{x}, h}\right)(\text { or } \neg \bar{x}<\bar{y})\right\} .
$$

(3) Why do we have clauses $(\mathrm{b})(\delta)$ and $(\mathrm{b})(\varepsilon)$ ? For the application here (b) $(\delta)$ suffices; if this is enough for the reader then clause $(\mathrm{J})$ in the definition of $\mathcal{A P}$ may be omitted. But they both look "local maximal".

Proof of 2.3(1). We will use (2). Let $T \subseteq \lambda^{+}>\lambda$ and $c, \bar{d}$ be as there and let all the functions $h_{\kappa, u} \in H_{\mu}^{0}$ defined in the continuation of the proof of 2.3(1) below be in $H_{\mu}^{1, *}$. We may assume that if $x \in T$ and $\alpha<\lambda$ then $x \frown\langle\alpha\rangle \in T$. Let $<^{\otimes}$ be a linear order on $\lambda$ such that $\left(\lambda,<^{\otimes}\right)$ has neither first nor last element and is $\lambda$-dense (i.e. if $\alpha_{i}<{ }^{\otimes} \beta_{j}$ for $i<i_{0}<\lambda$, $j<j_{0}<\lambda$ then $\alpha_{i} \leq^{\otimes} \gamma \leq^{\otimes} \beta_{j}$ for some $\gamma$ ). We define the order $<_{\mathcal{I}}$ on $\mathcal{I}=T^{\langle 1\rangle}=\{x \in T: \operatorname{lev}(x)$ is a successor or of cofinality $\lambda\}$ :

$y<_{\mathcal{I}} z \quad$ if and only if either $z=(y \wedge z) \triangleleft y$ or $y(\alpha))<^{\otimes} z(\alpha)$, where $\alpha=\operatorname{lev}(y \wedge z)$.

Clearly $\left(\mathcal{I},<_{\mathcal{I}}\right)$ is a dense linear order of density $\lambda^{+}$and size $\lambda^{+}$. To show that $\mathcal{I}$ has exact density $\lambda^{+}$(i.e. its exact density is $\left(\lambda^{+}, \lambda^{++}, \lambda^{+}\right)$) assume that $\mathcal{J} \subseteq \mathcal{I}$ and $|\mathcal{J}|=\lambda^{+}$. We want to show that $\mathcal{J}$ has density $\lambda^{+}$. Suppose that $\mathcal{J}_{0} \subseteq \mathcal{J}$ and $\left|\mathcal{J}_{0}\right| \leq \lambda$. Then $\mathcal{J}_{0} \subseteq \bigcup_{\alpha<\alpha(*)} T_{\alpha}$ for some $\alpha(*)<\lambda^{+}$, and we may find distinct $x, y \in \mathcal{J} \backslash \bigcup_{\alpha \leq \alpha(*)} T_{\alpha}$ such that $x\lceil\alpha(*)=y\lceil\alpha(*)$. Then $x, y$ show that $\mathcal{J}_{0}$ is not dense in $\mathcal{J}$.

Now we are proving that $\mathcal{I}$ satisfies $(*)_{2}$. Suppose that $\kappa<\mu$ and $\left(a_{i}, b_{i}\right)$ are disjoint intervals in $\mathcal{I}$ (for $i<\kappa$ ). Suppose that $\bar{y}_{\xi}=\left\langle y_{i}^{\xi}: i<\kappa\right\rangle$ (for $\left.\xi<\lambda^{+}\right)$are such that $a_{i}<_{\mathcal{I}} y_{i}^{\xi}<_{\mathcal{I}} b_{i}$ and $y_{i}^{\xi}$ 's are pairwise distinct for $\xi<$ $\lambda^{+}$. Let $u \subseteq \kappa$. Take $\alpha(*)<\lambda^{+}$such that $(\forall i<\kappa)\left(\operatorname{lev}\left(a_{i}\right), \operatorname{lev}\left(b_{i}\right)<\alpha(*)\right)$. As $y_{i}^{\xi}$ 's are pairwise distinct we may assume that

$$
\left(\forall \xi<\lambda^{+}\right)(\forall i<\kappa)\left(\alpha(*)<\operatorname{lev}\left(y_{i}^{\xi}\right) \text { and } \xi \leq \operatorname{lev}\left(y_{i}^{\xi}\right)\right) .
$$


Note that if $i<j<\kappa$ and $\xi, \zeta<\lambda^{+}$then $y_{i}^{\xi}\left\lceil\alpha(*) \neq y_{j}^{\zeta}\lceil\alpha(*)\right.$. Now the following claim is applicable to $\left\langle\left\langle y_{i}^{\xi}: i<\kappa\right\rangle: \xi \in[\alpha(*), \lambda)\right\rangle$ and as we shall see later this finishes the proof of 2.3(1) shortly.

Claim 2.3.1. Assume (for the objects constructed in 2.3(2)):

(a) $\kappa<\mu$,

(b) for each $\xi<\lambda^{+}$we have a sequence $\bar{y}^{\xi}=\left\langle y_{i}^{\xi}: i<\kappa\right\rangle$ such that $y_{i}^{\xi} \in T$ and either

$(\alpha)\left(\xi^{1}, i_{1}\right) \neq\left(\xi^{2}, i_{2}\right) \Rightarrow y_{i_{1}}^{\xi^{1}} \neq y_{i_{2}}^{\xi^{2}}$ or

( $\beta) \operatorname{lev}\left(y_{i}^{\xi}\right) \geq \xi$

(c) $h \in H_{\mu}^{1, *} \cup H_{\mu}^{3, *}$,

(d) for some $\alpha(*)<\lambda^{+}$,

$$
\xi, \zeta<\lambda^{+}, i<j<\kappa \Rightarrow y_{i}^{\xi}\left\lceil\alpha(*) \neq y_{j}^{\zeta}\lceil\alpha(*) .\right.
$$

Then we can find $\xi_{1}<\xi_{2}<\lambda^{+}$such that clause (b)( $\left.\delta\right)(\mathrm{i}) \quad($ or $(\mathrm{b})(\varepsilon)(\mathrm{i})$, respectively) of $2.3(2)$ holds with $\left(\bar{y}^{\xi_{1}}, \bar{y}^{\xi_{2}}\right)$ standing for $(\bar{y}, \bar{z})$, i.e.:

$(\delta)$ if $h \in H_{\mu}^{1, *}$ then

(i) $\left\langle y_{\varepsilon}^{\xi_{2}}(\operatorname{lev}(\bar{t})): \varepsilon<\kappa\right\rangle \in h\left(\left\langle c\left(t_{\varepsilon}\right), y_{\varepsilon}^{\xi_{1}}(\operatorname{lev}(\bar{t})): \varepsilon<\kappa\right\rangle\right.$,

( $\varepsilon)$ if $h \in H_{\mu}^{3, *}$ then

(i) $\left\langle\left(c\left(t_{\varepsilon}\right), y_{\varepsilon}^{\xi_{1}}(\operatorname{lev}(\bar{t})), y_{\varepsilon}^{\xi_{2}}(\operatorname{lev}(\bar{t}))\right): \varepsilon<\kappa\right\rangle \in h(\kappa)$,

where $\bar{t}=\left\langle t_{j}: j<\kappa\right\rangle$ and $t_{j}=y_{j}^{\xi_{1}} \wedge y_{j}^{\xi_{2}}$ etc.

Proof. First note that, by easy thinning (as either $(b)(\alpha)$ or $(b)(\beta)$ holds; remember clause (e)) we can assume $(\mathrm{b})(\alpha) \&(\mathrm{~b})(\beta)$. As $\lambda=\lambda^{\kappa}$ we may assume that $\left\langle y_{i}^{\delta}\lceil\alpha(*): i<\kappa\rangle\right.$ is the same for all $\delta \in \lambda^{+}$. Let

$$
\begin{aligned}
Z=\left\{\alpha \in\left[\alpha(*), \lambda^{+}\right):\right. & \\
& \left(\exists Y \in\left[T_{\alpha}\right]^{<\lambda}\right)\left(\mid\left\{\xi<\lambda^{+}:(\forall i<\kappa)\left(y_{i}^{\xi}\lceil\alpha \notin Y)\right\} \mid \leq \lambda\right)\right\} .
\end{aligned}
$$

First we are going to show that $Z \neq\left[\alpha(*), \lambda^{+}\right)$. If not then for each $\alpha \in$ $\left[\alpha(*), \lambda^{+}\right)$we find a set $Y_{\alpha} \in\left[T_{\alpha}\right]^{<\lambda}$ and an ordinal $\xi(\alpha)<\lambda^{+}$such that

$$
\left\{\xi<\lambda^{+}:(\forall i<\kappa)\left(y_{i}^{\xi}\lceil\alpha \notin Y)\right\} \subseteq \xi(\alpha) .\right.
$$

For $\alpha \in\left[\alpha(*), \lambda^{+}\right)$, choose $\bar{x}^{\alpha} \in T_{\alpha}^{[\mu]}$ such that:

(i) $(\forall i<\kappa)\left(y_{i}^{\alpha}\left\lceil\alpha=x_{i}^{\alpha}\right)\right.$,

(ii) $Y_{\alpha} \subseteq\left\{x_{i}^{\alpha}: i<\mu\right\}$.

For each $\delta \in\left[\alpha(*), \lambda^{+}\right)$with $\operatorname{cf}(\delta)=\lambda$ we can find $\gamma_{\delta}<\delta$ such that $\bar{x}_{\delta}^{*}=$ $\left\langle x_{i}^{\delta}\left\lceil\gamma_{\delta}: i<\mu\right\rangle\right.$ is with no repetition (recall that $x_{i}^{\delta} \in T_{\delta}$ are pairwise distinct, $i<\mu<\lambda$ and the tree $T$ is normal). By Fodor's lemma, for some $\gamma^{*}$ the set

$$
S_{0}:=\left\{\delta \in\left[\alpha(*), \lambda^{+}\right): \operatorname{cf}(\delta)=\lambda \& \gamma_{\delta}=\gamma^{*}\right\}
$$


is stationary. For $\delta \in S_{0}$ there are at most $\lambda^{\mu}=\lambda$ possibilities for $\left\langle x_{i}^{\delta} \uparrow \gamma^{*}\right.$ : $i<\mu\rangle$ and hence for some $\bar{x}^{*}=\left\langle x_{i}^{*}: i<\mu\right\rangle \in T_{\gamma^{*}}^{[\mu]}$ the set

$$
S_{1}:=\left\{\delta \in S_{0}: \bar{x}^{*}=\left\langle x_{i}^{\delta}\left\lceil\gamma^{*}: i<\mu\right\rangle\right\}\right.
$$

is stationary. Hence the set

$$
S_{2}:=\left\{\delta \in\left[\alpha(*), \lambda^{+}\right) \cap S_{1}:(\forall i<\mu)\left(x_{i}^{\delta}\left\lceil\gamma^{*}=x_{i}^{*}\right) \&(\forall \alpha<\delta)(\xi(\alpha)<\delta)\right\}\right.
$$

is stationary. Look at $d_{\bar{x}^{*}, h}$ (really any $h^{\prime} \in H_{\mu}^{l, *}$ will do here). Note that $\bar{x}^{\delta} \in \operatorname{dom}\left(d_{\bar{x}^{*}, h}\right)$ for $\delta \in S_{2}$ (remember $\operatorname{cf}(\delta)=\lambda$ ), and therefore we find $\delta_{1}, \delta_{2} \in S_{2}$ with $\delta_{1}<\delta_{2}$ such that $d_{\bar{x}^{*}, h}\left(\bar{x}^{\delta_{1}}\right)=d_{\bar{x}^{*}, h}\left(\bar{x}^{\delta_{2}}\right)$. As $\xi\left(\delta_{1}\right)<\delta_{2}$ we can find $i<\kappa$ such that $y_{i}^{\delta_{2}}\left\lceil\delta_{1} \in Y_{\delta_{1}}\right.$. Thus for some $j<\mu$ necessarily $y_{i}^{\delta_{2}}\left\lceil\delta_{1}=x_{j}^{\delta_{1}}\right.$ and hence $x_{i}^{\delta_{2}}\left\lceil\delta_{1}=x_{j}^{\delta_{1}}\right.$, and consequently $x_{i}^{\delta_{2}}\left\lceil\gamma^{*}=x_{j}^{\delta_{1}}\left\lceil\gamma^{*}\right.\right.$. This implies $i=j$ (as $\delta_{1}, \delta_{2} \in S_{2} \subseteq S_{0}$ and hence $\gamma_{\delta_{1}}=$ $\gamma^{*}=\gamma_{\delta_{2}}$ and now apply the definition of $\left.\gamma_{\delta_{1}}, \gamma_{\delta_{2}}, S_{2}\right)$ and thus $x_{i}^{\delta_{1}} \triangleleft x_{i}^{\delta_{2}}$, which contradicts clause $(\beta)$ of $2.3(2 \mathrm{~b})$. So we have proved $Z \neq\left[\alpha(*), \lambda^{+}\right)$. But by its definition $Z$ is an initial segment of $\left[\alpha(*), \lambda^{+}\right)$. Hence for some $\beta(*) \in\left[\alpha(*), \lambda^{+}\right)$we have $Z=[\alpha(*), \beta(*))$.

Let $\beta \in\left[\beta(*), \lambda^{+}\right)$be a successor ordinal. By induction on $\varepsilon<\mu$ choose $\left(^{3}\right)$ pairwise disjoint $\bar{x}^{\varepsilon}=\left\langle x_{i}^{\varepsilon}: i<\kappa\right\rangle \in T_{\beta}^{[\kappa]}$ such that the sets

$$
Z_{\varepsilon}:=\left\{\xi \in\left[\beta, \lambda^{+}\right):(\forall i<\kappa)\left(y_{i}^{\xi} \uparrow \beta=x_{i}^{\varepsilon}\right)\right\}
$$

are of size $\lambda^{+}$. Suppose we have defined $\bar{x}^{\varepsilon}$ for $\varepsilon<\varepsilon^{\prime}$. Let $Y=\left\{x_{i}^{\varepsilon}\right.$ : $\left.i<\kappa, \varepsilon<\varepsilon^{\prime}\right\}$. Then $Y \in\left[T_{\beta}\right]^{<\lambda}$ and by the choice of $\beta$ the set $\left\{\xi<\lambda^{+}\right.$: $(\forall i<\kappa)\left(y_{i}^{\xi}\lceil\beta \notin Y)\right\}$ is of size $\lambda^{+}$. As $\lambda^{\kappa}=\lambda$ we can find $\bar{x}^{\varepsilon^{\prime}} \in T_{\beta}^{[\kappa]}$ such that

$$
\left|\left\{\xi<\lambda^{+}:(\forall i<\kappa)\left(y_{i}^{\xi} \uparrow \beta=x_{i}^{\varepsilon^{\prime}} \notin Y\right)\right\}\right|=\lambda^{+} .
$$

Now for $\varepsilon<\mu$ and $i<\kappa$ let $x_{\kappa \cdot \varepsilon+i}=x_{i}^{\varepsilon}$ and let $\bar{x}=\left\langle x_{j}: j<\mu\right\rangle$. Thus $\bar{x} \in T_{\beta}^{[\mu]}$. For each $\alpha \in\left[\beta, \lambda^{+}\right)$choose $\left\langle\zeta_{\alpha, \varepsilon}: \varepsilon<\mu\right\rangle$ such that $\zeta_{\alpha, \varepsilon} \in Z_{\varepsilon}$ and $\alpha_{1}<\alpha_{2}$ implies $\alpha_{1}<\zeta_{\alpha_{1}, \varepsilon}<\zeta_{\alpha_{2}, \varepsilon}$. For $\varepsilon<\mu, i<\kappa$ and $\alpha \in\left[\beta, \lambda^{+}\right)$put $z_{\kappa \cdot \varepsilon+i}^{\alpha}=y_{i}^{\zeta_{\alpha, \varepsilon}} \mid \alpha$. Then $\bar{z}^{\alpha}=\left\langle z_{j}^{\alpha}: j<\mu\right\rangle \in T_{\alpha}^{[\mu]}$ and $\bar{x}<\bar{z}^{\alpha}$. Consider the function $d_{\bar{x}, h}$. Let $S_{3}:=\left\{\delta \in\left(\beta, \lambda^{+}\right): \operatorname{cf}(\delta)=\lambda\right\}$ and note that for each $\alpha \in S_{3}$ the restriction $d_{\bar{x}, h} \uparrow\left\{\bar{z}^{\alpha} \uparrow \gamma: \beta<\gamma \leq \alpha\right\}$ is a one-to-one function (since $\operatorname{cf}(\alpha)=\lambda$ and we have clause $(\beta)$ of $2.3(2 \mathrm{~b})$ ). Consequently, for $\alpha \in S_{3}$, we find $\delta(\alpha)$ such that

$$
\delta(\alpha) \leq \delta<\alpha \Rightarrow d_{\bar{x}, h}\left(\bar{z}^{\alpha}\lceil\delta)>d_{\bar{x}, h}\left(\bar{z}^{\alpha}\right), \quad \delta(\alpha)>\beta .\right.
$$

Next, applying Fodor's lemma, we find a stationary set $S_{4}^{\prime} \subseteq S_{3}$ and $\delta^{*}>\beta$ such that $d_{\bar{x}, h}\left(\bar{z}^{\alpha}\lceil\delta)>d_{\bar{x}, h}\left(\bar{z}^{\alpha}\right)\right.$ for $\alpha \in S_{4}^{\prime}$ and $\delta \in\left(\delta^{*}, \alpha\right)$, and $\bar{z}^{\alpha_{1}}\left\lceil\delta^{*}=\right.$

\footnotetext{
$\left({ }^{3}\right)$ We could have done it for $\varepsilon<\lambda$, but it is not necessary here.
} 
$\bar{z}^{\alpha_{2}} \mid \delta^{*}$ for all $\alpha_{1}, \alpha_{2} \in S_{4}^{\prime}$. Let

$$
S_{4}:=\left\{\delta \in S_{4}^{\prime}:(\forall \alpha<\delta)\left(\mid\left\{\beta \in S_{4}^{\prime}: \bar{z}^{\beta}\left\lceil\alpha=\bar{z}^{\delta}\lceil\alpha\} \mid=\lambda^{+}\right)\right\} .\right.\right.
$$

If $S_{4}$ is not stationary then for $\delta \in S_{4}^{\prime} \backslash S_{4}$ choose $\alpha_{\delta}<\delta$ contradicting the demand in the definition of $S_{4}$. For some stationary $S^{*} \subseteq S_{4}^{\prime} \backslash S_{4}$ we have $\alpha_{\delta}=\alpha^{*}$ for $\delta \in S^{*}$ and we get an easy contradiction.

As $\operatorname{rang}\left(d_{\bar{x}, h}\right) \subseteq \lambda$, for some stationary $S_{5} \subseteq S_{4}, d_{\bar{x}, h}\left\lceil\left\{\bar{z}^{\alpha}: \alpha \in S_{5}\right\}\right.$ is constant. Choose $\alpha_{1} \neq \alpha_{2}$ from $S_{5}$. By clauses $(\alpha),(\beta)$ of $2.3(2 \mathrm{~b})$ we see that if $t_{j}=z_{j}^{\alpha_{1}} \wedge z_{j}^{\alpha_{2}}($ for $j<\mu)$ then $\bar{t} \in T_{\bar{x}}^{[\mu]}$ and $\operatorname{lev}(\bar{x}) \leq \operatorname{lev}(\bar{t})<\operatorname{lev}\left(\bar{z}^{\alpha_{1}}\right)$, $\operatorname{lev}(\bar{t})<\operatorname{lev}\left(\bar{z}^{\alpha_{2}}\right)$. Moreover, by the definition of $S_{4}^{\prime}$ we have $\operatorname{lev}(\bar{t}) \geq \delta^{*}$ and $d_{\bar{x}, h}(\bar{t})>d_{\bar{x}, h}\left(\bar{z}^{\alpha_{1}}\right)$. Hence by clause $(\delta)$ of $2.3(2 \mathrm{~b})$ we find $i<\mu$ divisible by $\kappa$ such that either (i) or (ii) of clause $(\delta)$ of $2.3(2 \mathrm{~b})$ holds with $(\bar{y}, \bar{z})$ there standing for $\left(\bar{z}^{\alpha_{1}}, \bar{z}^{\alpha_{2}}\right)$ here. By symmetry, without loss of generality, (i) of $2.3(2 \mathrm{~b})(\delta)$ holds. Let $\operatorname{lev}(\bar{t})<\beta_{l}<\alpha_{l}$ (for $\left.l=1,2\right)$. Hence by the definition of $S_{4}$ (and as $\alpha_{2} \in S_{5} \subseteq S_{4}$ ) and by the character of the requirement on $\alpha_{1}, \alpha_{2}$ we have, without loss of generality, $\alpha_{1}<\alpha_{2}$, so we are done. $\mathbf{m}_{2.3 .1}$

Continuation of the proof of 2.3(1). Remember we have $\kappa<$ $\mu,\left(a_{i}, b_{i}\right)$ (for $\left.i<\mu\right), u \subseteq \kappa$ and $\left\langle\bar{y}^{\xi}: \xi \in\left[\alpha(*), \lambda^{+}\right)\right\rangle$with $\bar{y}^{\xi}=\left\langle y_{i}^{\xi}: i<\kappa\right\rangle$. Define $h=h_{\kappa, u} \in H_{\mu}^{0}$ (and assume that for each $\kappa<\mu$ and $u \subseteq \kappa$ we have $\left.h_{\kappa, u} \in H_{\mu}^{1, *}\right)$ :

$$
h\left(\left\langle\beta_{i}^{1}: i<\kappa\right\rangle\right)=\left\{\left\langle\beta_{i}^{2}: i<\kappa\right\rangle \in{ }^{\kappa} \lambda:(\forall i<\kappa)\left(\beta_{i}^{1}<{ }^{\otimes} \beta_{i}^{2} \Leftrightarrow i \in u\right)\right\} .
$$

By the choice of $<^{\otimes}$ it is easy to check that $h \in H_{\mu}^{0}$. So by Claim 2.3.1 for $\kappa, h, \bar{y}^{\xi}$, there are $\alpha^{1}<\alpha^{2}$ as there and we are done.

We still have to prove $(*)_{1}$. Suppose that $a, b \in \mathcal{I}$ and $a<_{\mathcal{I}} b$. By the definition of the order there is $t \in T$ such that $t \unlhd s \in T \Rightarrow s \in(a, b)_{\mathcal{I}}$. As the tree $T$ is $\lambda^{+}$-Aronszajn we find $\bar{x} \in T_{\alpha}^{[\mu]}$ (for some $\alpha \in\left(\operatorname{lev}(t), \lambda^{+}\right)$) such that $(\forall j<\mu)\left(t \unlhd x_{j}\right)$. Next for every $\beta \in\left(\alpha, \lambda^{+}\right)$we can choose $\bar{y}_{\beta} \in T_{\beta}^{[\mu]}$ such that $\bar{x}<\bar{y}_{\beta}$. Take any $h \in H_{\mu}^{1, *} \cup H_{\mu}^{3, *}$. For some unbounded $S \subseteq\left(\alpha, \lambda^{+}\right)$the sequence $\left\langle d_{\bar{x}, h}\left(\bar{y}_{\beta}\right): \beta \in S\right\rangle$ is constant. Consequently, elements $y_{0}^{\beta}$ for $\beta \in S$ are pairwise $\triangleleft$-incomparable (in the tree $T$ ). Hence $\left\{\left\{y \in T: y_{0}^{\beta} \unlhd y\right\}: \beta \in S\right\}$ is a family of pairwise disjoint convex subsets of $(a, b)_{\mathcal{I}}$ (each with $\lambda^{+}$elements), so we have finished. $\mathbf{q}_{2.3(1)}$

Pro of of 2.3(2). We want to apply [Sh 405, Appendix]. For this we have to define a set $\mathcal{A P}$ of approximations and check the conditions of 2.1.

Definition 2.3.2. The set $\mathcal{A P}$ of approximations consists of all tuples $p=\langle t, w, \leq, D, \bar{d}, \bar{e}, f, c\rangle$ (we may write $t^{p}, w^{p}$ etc.) such that:

(A) $t$ is a subset of $\lambda^{+}$of cardinality $<\lambda, t \cap[0, \lambda)=\{0\}$, 
(B) $w=\left\{\alpha<\lambda^{+}:[\lambda \cdot \alpha, \lambda \cdot(\alpha+1)) \cap t \neq \emptyset\right\}$ is such that

$$
\left(\forall \alpha<\lambda^{+}\right)(\alpha \in w \Leftrightarrow \alpha+1 \in w),
$$

and the set $\left\{\alpha<\lambda^{+}: \omega \cdot \alpha \in w\right\}$ is closed,

(C) $\leq=\leq_{t}$ is a partial order on $t$ such that $t=t^{p}=(t, \leq)$ is a normal tree (so $x \wedge y$ is well defined) and for each $\alpha \in w$ the set $t \cap[\lambda \cdot \alpha, \lambda \cdot(\alpha+1)$ ) is the $\operatorname{otp}(w \cap \alpha)$ th level of $t$ (but possibly $\alpha<\beta$ are in $w$, and for some $x$ in the $\alpha$ th level of $t$ there is no $y$ in the $\beta$ th level, $x \leq_{t} y$ ) [obviously, the intention is: $t$ approximates $T$; we may use $t$ for $\left.\left(t, \leq_{t}\right)\right]$,

(D) $D$ is a set of $<\lambda$ pairs $(\bar{x}, h)$ such that $\bar{x} \in t^{[\mu]}$ and $h \in H_{\mu}^{1, *} \cup H_{\mu}^{3, *}$,

(E) $\bar{d}=\left\langle d_{\bar{x}, h}:(\bar{x}, h) \in D\right\rangle$, each $d_{\bar{x}, h}$ is a partial function from $t_{\bar{x}}^{[\mu]}$ to $\lambda$ with domain of cardinality $<\lambda$ such that

$$
\left[\bar{y} \in t_{\bar{x}}^{[\mu]} \& \bar{x}<_{t} \bar{y}<_{t} \bar{z} \& \bar{z} \in \operatorname{dom}\left(d_{\bar{x}, h}\right)\right] \Rightarrow \bar{y} \in \operatorname{dom}\left(d_{\bar{x}, h}\right),
$$

(F) $\bar{e}=\left\langle e_{\bar{x}, h}:(\bar{x}, h) \in D\right\rangle$, each $e_{\bar{x}, h}$ is a partial function from $t_{\bar{x}}^{[\mu]} \times \lambda$ to $\{0,1,2\}$ of size $<\lambda$ and such that:

(i) $(\bar{y}, \gamma) \in \operatorname{dom}\left(e_{\bar{x}, h}\right) \Rightarrow \bar{y} \in \operatorname{dom}\left(d_{\bar{x}, h}\right)$, and

$$
\begin{aligned}
& \operatorname{dom}\left(e_{\bar{x}, h}\right) \\
& \quad \supseteq\left\{(\bar{y}, \gamma): \bar{y} \in \operatorname{dom}\left(d_{\bar{x}, h}\right) \&\left(\exists \bar{z} \in \operatorname{dom}\left(d_{\bar{x}, h}\right)\right)\left(\gamma \leq d_{\bar{x}, h}(\bar{z})\right)\right\},
\end{aligned}
$$

(ii) if $\bar{y} \in t_{\bar{x}}^{[\mu]}, \bar{x}<_{t} \bar{y}<_{t} \bar{z} \in \operatorname{dom}\left(d_{\bar{x}, h}\right)$ and $e_{\bar{x}, h}(\bar{z}, \beta)$ is defined then $e_{\bar{x}, h}(\bar{y}, \beta)$ is defined and $e_{\bar{x}, h}(\bar{y}, \beta) \leq e_{\bar{x}, h}(\bar{z}, \beta)$, and if $\bar{y} \in t_{\bar{x}}^{\langle\mu\rangle}$ then at most one of them is 1 [here, we interpret $t_{\bar{x}}^{\langle\mu\rangle}$ as the set of those $\bar{y} \in t_{\bar{x}}^{[\mu]}$ such that $\operatorname{lev}(\bar{y})$ is either a successor ordinal or is $\operatorname{otp}(w \cap \alpha)$ for some $\alpha \in w$ such that $\operatorname{cf}(\alpha)=\lambda$ ],

(iii) $d_{\bar{x}, h}(\bar{y})=\alpha \Leftrightarrow e_{\bar{x}, h}(\bar{y}, \alpha)=1$,

[the intention: $e_{\bar{x}, h}$ is not explicitly present in $2.3(2 \mathrm{~b})$, but $e_{\bar{x}, h}(\bar{y}, \gamma)=l$ will mean that: if $l=0$ then for some $\bar{z}$ we have $\bar{y}<\bar{z}$ and $d_{\bar{x}, h}(\bar{z})=\gamma$; if $l=1$ then $d_{\bar{x}, h}(\bar{y})=\gamma$ and if $l=2$ then none of these],

(G) $f$ is a function from $t^{+}=\{\alpha \in t: \alpha$ is of a successor level in $t\}$ to $\lambda$ such that if $\gamma \neq \beta$ are immediate successors (in $t$ ) of some $\alpha$ then $f(\beta) \neq f(\gamma)$ [the intention is that if $\alpha$ represents $\eta \in T_{i+1}$, then $f(\alpha)=\eta(i)$ ],

(H) $c$ is a function from $t$ to $\lambda$,

(I) if $(\bar{x}, h) \in D, e_{\bar{x}, h}(\bar{y}, \alpha), e_{\bar{x}, h}(\bar{z}, \alpha) \leq 1$ and $\neg\left[\bar{y} \leq_{t} \bar{z}\right]$ and $\neg\left[\bar{z} \leq_{t} \bar{y}\right]$ then clauses $(\alpha)-(\varepsilon)$ of $2.3(2 \mathrm{~b})$ hold (with $\left\langle f\left(y_{\varepsilon} \uparrow(i+1)\right), f\left(z_{\varepsilon} \uparrow(i+1)\right)\right\rangle$ replacing $\left.\left\langle y_{\varepsilon}(i), z_{\varepsilon}(i)\right\rangle\right)$ and $\alpha$ in place of $\left.d_{\bar{x}, h}(\bar{y})=d_{\bar{x}, h}(\bar{z})\right)$,

$(\mathrm{J})$ if $\alpha \in w^{p}, \bar{x}<\bar{y}^{0}<\bar{y}^{1}$, all in $\left(t^{p}\right)^{[\mu]},(\bar{x}, h) \in D, h \in H_{\mu}^{3, *}$ and $\bar{y}^{0}, \bar{y}^{1} \in \operatorname{dom}\left(d_{\bar{x}, h}\right), \operatorname{lev}\left(\bar{y}^{0}\right)=\alpha, \operatorname{lev}\left(\bar{y}^{1}\right)=\alpha+1, e_{\bar{x}, h}\left(\bar{y}^{1}, \gamma\right) \leq 1$ and $\gamma<d_{\bar{x}, h}\left(\bar{y}^{0}\right)$ then in looking at $\bar{y}^{0}, \bar{y}^{1}$ as candidates for $\bar{t}, \bar{y}$ (or $\bar{t}, \bar{z}$ ) in clause (I) (i.e. in clauses $(\alpha)-(\varepsilon)$ of $2.3(2 \mathrm{~b})$ ) in $(\varepsilon)$ there, for each $\zeta<\mu$, for $\mu$ 
ordinals $i<\mu$ divisible by $\zeta$, the values we have, i.e. $c^{p}\left(y_{i+\varepsilon}^{0}\right), f^{p}\left(y_{i+\varepsilon}^{1}\right)$ for $\varepsilon<\zeta$, are compatible with the demand (i) there, i.e. for every $\beta<\lambda$ there are $\alpha_{\varepsilon}^{2} \in(\beta, \lambda)$ for $\varepsilon<\zeta$ such that

$$
\left\langle\left(c^{p}\left(y_{i+\varepsilon}^{0}\right), f^{p}\left(y_{i+\varepsilon}^{1}\right), \alpha_{\varepsilon}^{2}\right): \varepsilon<\zeta\right\rangle \in h(\zeta),
$$

$(\mathrm{K})$ if $\bar{x}<\bar{y}$ are in $t^{[\mu]},(\bar{x}, h) \in D, \bar{y} \in \operatorname{dom}\left(d_{\bar{x}, h}\right), \alpha<d_{\bar{x}, h}(\bar{y})$ and $e_{\bar{x}, h}(\bar{y}, \alpha)=0$ then $(\forall \varepsilon<\mu)\left(\exists^{\mu} i<\mu\right)\left(c\left(y_{i}\right)=\varepsilon\right)$ (i.e. looking at $\bar{y}$ as a candidate for $\bar{t}$ in $2.3(2 \mathrm{~b})(\gamma)$ the values we have are compatible with the demand there).

The set $\mathcal{A P}$ of approximations is equipped with the natural partial order.

We will want to apply the machinery of [Sh 405, Appendix] to the partial order $(\mathcal{A P}, \leq)$. For this we have to represent it as a standard $\lambda^{+}$semiuniform partial order. In representing it as a partial order on $\lambda \times\left[\lambda^{+}\right]^{<\lambda}$ we define the set of terms such that:

(a) $\{\tau(u): \tau$ a term with otp $(u)$ places $\}=\left\{p \in \mathcal{A P}:\left\{\alpha<\lambda^{+}: \omega \cdot \alpha \in\right.\right.$ $\left.\left.w^{p}\right\}=u\right\}$, for a closed set $u \in\left[\lambda^{+}\right]^{<\lambda}$,

(b) if $p_{l}=\tau\left(u_{l}\right)$ for $l=1,2$ then $\operatorname{otp}\left(t^{p_{1}}, \leq\right)=\operatorname{otp}\left(t^{p_{2}}, \leq\right)$ and the one-to-one order preserving mapping $g$ from $t^{p_{1}}$ onto $t^{p_{2}}$ maps $p_{1}$ to $p_{2}$ (i.e. $\alpha \leq^{p_{1}} \beta \Leftrightarrow g(\alpha) \leq{ }^{p_{2}} g(\beta)$, etc. $)$.

Note that for $p \in \mathcal{A P}$, its domain $\operatorname{dom}(p)$ (in the sense of 2.1) is $\{\alpha$ : $\left.\omega \cdot \alpha \in w^{p}\right\}$. Hence, $\mathcal{A P}_{\alpha}=\left\{p \in \mathcal{A P}: w^{p} \subseteq \omega \cdot \alpha\right\}$.

Now we have to check that (with this representation) $\mathcal{A} \mathcal{P}$ satisfies the demands 2.1(2)(a)-(h). Clauses (a) and (c) there should be clear.

To deal with clause (b) of 2.1(2), for an approximation $p \in \mathcal{A P}$ and $\alpha<\lambda^{+}$such that either $\alpha$ is a successor ordinal or $\operatorname{cf}(\alpha)=\lambda$, we define $q=p\lceil\omega \cdot \alpha$ by:

- $t^{q}=t^{p} \cap \lambda \cdot(\omega \cdot \alpha), w^{q}=w^{p} \cap \omega \cdot \alpha, \leq^{q}=\leq^{p}\left\lceil t^{q}\right.$,

- $D^{q}=\left\{(\bar{x}, h) \in D^{p}: \bar{x} \subseteq t^{q}\right\}$,

- if $(\bar{x}, h) \in D^{q}$ then $d_{\bar{x}, h}^{q}=d_{\bar{x}, h}^{p}\left\lceil\left(t^{q}\right)_{\bar{x}}^{[\mu]}\right.$ and $e_{\bar{x}, h}^{q}=e_{\bar{x}, h}^{p}\left\lceil\left(\left(t^{q}\right)_{\bar{x}}^{[\mu]} \times \lambda\right)\right.$,

- $f^{q}=f^{p}\left\lceil\left(t^{q} \cap \operatorname{dom}\left(f^{q}\right)\right)\right.$ and $c^{q}=c^{p}\left\lceil t^{q}\right.$.

Observation 2.3.3. If $p \in \mathcal{A P}$ is an approximation and $\alpha<\lambda^{+}$is either a successor or of cofinality $\lambda$ then $p\lceil\omega \alpha \in \mathcal{A P}$ is a unique maximal approximation such that $p\left\lceil\omega \cdot \alpha \leq p\right.$ and $w^{p \nmid \omega \cdot \alpha}=w^{p} \cap \omega \cdot \alpha$.

Thus $p\lceil\omega \cdot \alpha$ corresponds to $p\lceil\alpha$ as required in 2.1(2c). The main difficulty of the proof is checking the amalgamation property $2.1(2 \mathrm{~d})$. Before we deal with this demand we will check that some sets are dense in $\mathcal{A P}$ (which will allow us to simplify some arguments and will be of importance in drawing conclusions) and we will deal with existence of some upper bounds. 
Claim 2.3.4. In $\mathcal{A P}$, if $\left\langle p_{i}: i<\delta\right\rangle$ is increasing, $\delta<\lambda$ and for $i_{0}<i_{1}<$ $\delta$ we have $w^{p_{i_{0}}}=w^{p_{i_{1}}}$ then its union (defined naturally) is its least upper bound in $\mathcal{A P}$.

Claim 2.3.5 (Density Observation). Assume $p \in \mathcal{A P}$.

(1) Suppose that $\alpha \in w^{p}, u \subseteq[\lambda \cdot \alpha, \lambda \cdot \alpha+\lambda) \backslash t_{p},|u|<\lambda$ and for $i \in u$ we are given a full branch $A_{i}$ of $\left(t^{p} \cap \lambda \cdot \alpha, \leq^{p}\right)$ (i.e. $A_{i}$ is linearly ordered by $\leq^{p}$ and $\left.\beta \in w^{p} \cap \alpha \Rightarrow[\lambda \cdot \beta, \lambda \cdot \beta+\lambda) \cap A_{i} \neq \emptyset\right), i \neq j \Rightarrow A_{i} \neq A_{j}$. Furthermore, assume that if $\alpha$ is limit then

$$
\gamma \in t^{p} \cap[\lambda \cdot \alpha, \lambda \cdot \alpha+\lambda) \& i \in u \Rightarrow A_{i} \neq\left\{y \in t^{p}: y<^{p} \gamma\right\} .
$$

Then there is $q \in \mathcal{A P}$ with $p \leq q$ such that $t^{q}=t^{p} \cup u,<^{q}=<^{p} \cup\{(y, i)$ : $\left.y \in A_{i}, i \in u\right\}$, and the rest is equal (i.e. $w^{q}=w^{p}, D^{q}=D^{p}, \bar{d}^{q}=\bar{d}^{p}$, $\bar{e}^{q}=\bar{e}^{p}, f^{q} \supseteq f^{p}, c^{q} \supseteq c^{p}$ naturally).

(2) If $\alpha \in w^{p}$ and $i \in[\lambda \cdot \alpha, \lambda \cdot \alpha+\lambda)$ then there is $q \in \mathcal{A P}$ with $p \leq q$ such that $i \in t^{q}, w^{p}=w^{q}, D^{q}=D^{p}, \bar{d}^{q}=\bar{d}^{p}, \bar{e}^{q}=e^{p}$, and naturally $f^{q} \supseteq f^{p}, c^{q} \supseteq c^{p}$.

(3) If $\bar{x} \in\left(t^{p}, \leq^{p}\right)^{[\mu]}$ and $h \in H_{\mu}^{1, *} \cup H_{\mu}^{3, *}$ then there is $q \in \mathcal{A P}$ such that $p \leq q$ and $D^{q}=D^{p} \cup\{(\bar{x}, h)\}$.

(4) If $(\bar{x}, h) \in D^{p}$ and $\bar{x}<\bar{y} \in\left(t^{p}, \leq^{p}\right)^{[\mu]}$ then for some $q \in \mathcal{A P}$ with $p \leq q$ we have: $\bar{y} \in \operatorname{dom}\left(d_{\bar{x}, h}^{q}\right)=\operatorname{dom}\left(d_{\bar{x}, h}^{p}\right) \cup\left\{\bar{y}^{\prime}: \bar{x}<\bar{y}^{\prime} \leq \bar{y}, \bar{y}^{\prime} \in\left(t^{p}, \leq^{p}\right)^{[\mu]}\right\}$, $t^{p}=t^{q}, w^{p}=w^{q}, \leq^{p}=\leq^{q}, D^{p}=D^{q}, d_{\bar{x}^{\prime}, h^{\prime}}^{q}=d_{\bar{x}^{\prime}, h^{\prime}}^{p}, e_{\bar{x}^{\prime}, h^{\prime}}^{q}=e_{\bar{x}^{\prime}, h^{\prime}}^{p}$ for $\left(\bar{x}^{\prime}, h^{\prime}\right) \in D^{p} \backslash\{(\bar{x}, h)\}$, and $f^{q}=f^{p}, c^{q}=c^{p}$.

Proof. (1) Check.

(2) Iterate (1) (the $j$ th time - on the $j$ th level of $t$ ) using 2.3 .4 for the limit stage. More elaborately, for each $\beta \in w^{p} \backslash\{0\}$ choose $i_{\beta} \in[\lambda \cdot \beta, \lambda \cdot \beta+\lambda) \backslash t^{p}$ such that $i_{\alpha}=i$. Next by induction on $\beta \in w^{p} \cap(\alpha+1)$ choose an increasing sequence $\left\langle p_{\beta}: \beta \in w^{p} \cap(\alpha+1)\right\rangle \subseteq \mathcal{A P}$ of approximations such that $p_{0}=p$ and $t^{p_{\beta}}=t^{p} \cup\left\{i_{\gamma}: 0<\gamma \in w^{p} \cap(\beta+1)\right\}$ (so $w^{p_{\beta}}=w^{p}$ ) and the sequence $\left\langle i_{\gamma}: 0<\gamma \in w^{p} \cap(\beta+1)\right\rangle$ is $\leq^{p_{\beta}}$-increasing.

(3) We just put $t^{q}=t^{p}, w^{q}=w^{p}, f^{q}=f^{p}, c^{q}=c^{p}, d_{\bar{x}^{\prime}, h^{\prime}}^{q}=d_{\bar{x}^{\prime}, h^{\prime}}^{p}$ if $\left(\bar{x}^{\prime}, h^{\prime}\right) \in D^{p}, d_{\bar{x}, h}^{q}$ is empty if $(\bar{x}, h) \notin D^{p}$, and similarly for $e_{\bar{x}^{\prime}, h^{\prime}}^{q}$

(4) Let $\left\{\bar{y}_{\varepsilon}: \varepsilon<\zeta\right\}$ list $\operatorname{dom}\left(d_{\bar{x}, h}^{q}\right) \backslash \operatorname{dom}\left(d_{\bar{x}, h}^{p}\right)$ in the $<^{p}$-increasing way and let $\alpha^{*}=\sup \left(\operatorname{rang}\left(d_{\bar{x}, h}^{p}\right) \cup\left\{\gamma:\left(\exists \bar{y}^{\prime}\right)\left(\left(\bar{y}^{\prime}, \gamma\right) \in \operatorname{dom}\left(e_{\bar{x}, h}^{p}\right)\right)\right\}\right)$. Now put

$$
\operatorname{dom}\left(e_{\bar{x}, h}^{q}\right)=\left\{(\bar{y}, \alpha): \bar{y} \in \operatorname{dom}\left(d_{\bar{x}, h}^{q}\right) \& \alpha<\alpha^{*}+1+\zeta\right\},
$$

declare that $e_{\bar{x}, h}^{p} \subseteq e_{\bar{x}, h}^{q}$ and

- if $\bar{y} \in \operatorname{dom}\left(d_{\bar{x}, h}^{q}\right), \bar{y}<\bar{y}_{\varepsilon}, \varepsilon<\zeta$ then $e_{\bar{x}, h}^{q}\left(\bar{y}, \alpha^{*}+1+\varepsilon\right)=0$,

- $e_{\bar{x}, h}^{q}\left(\bar{y}_{\varepsilon}, \alpha^{*}+1+\varepsilon\right)=1$,

- $e_{\bar{x}, h}^{q}(\bar{y}, \gamma)=2$ in all other instances. 
It should be clear that this defines correctly an approximation $q \in \mathcal{A P}$ and that it is as required. [Note that clauses $(\gamma),(\varepsilon)$ of $2.3(2 \mathrm{~b})$ relevant to clauses $(\mathrm{J}),(\mathrm{K})$ in the definition of $\mathcal{A P}$ hold by the requirement " $d_{\bar{x}, h}(\bar{t})<$ $\left.d_{\bar{x}, h}(\bar{y})=d_{\bar{x}, h}(\bar{z}) ".\right] \mathbf{m}_{2.3 .5}$

Claim 2.3.6. If $p \in \mathcal{A P}$ and an ordinal $\alpha \in \lambda^{+} \backslash w^{p}$ is divisible by $\omega$ then for some $q \in \mathcal{A P}$ with $p \leq q$ we have $w^{q}=w^{p} \cup[\alpha, \alpha+\omega), D^{p}=D^{q}$ and

$$
\beta \in w^{p} \Rightarrow t^{q} \cap[\lambda \cdot \beta, \lambda \cdot \beta+\lambda)=t^{p} \cap[\lambda \cdot \beta, \lambda \cdot \beta+\lambda) .
$$

$\operatorname{Proof}$. Let $\beta=\min \left(w^{p} \backslash \alpha\right)$ (if $\beta$ is undefined then it is much easier; of course $\beta>\alpha$ as $\alpha \notin w^{p}$ and therefore $\left.\beta \geq \alpha+\omega\right)$. Let $t^{p} \cap[\lambda \cdot \beta, \lambda \cdot \beta+\lambda)=$ $\left\{y_{i}^{\beta}: i<i^{*}\right\}$ be an enumeration with no repetitions and for $n<\omega$ let

$$
\left\{y_{i}^{\alpha+n}: i<i^{*}\right\} \subseteq[\lambda \cdot(\alpha+n), \lambda \cdot(\alpha+n)+\lambda)
$$

be with no repetition. Let $t^{q}=t^{p} \cup\left\{y_{i}^{\alpha+n}: n<\omega, i<i^{*}\right\}$, and

$$
\begin{aligned}
\leq^{q}=\leq^{p} & \cup\left\{\left(y_{i}^{\alpha+n}, y_{i}^{\alpha+m}\right): n \leq m<\omega, i<i^{*}\right\} \\
& \cup\left\{\left(y_{i}^{\alpha+n}, x\right): n<\omega, i<i^{*}, y_{i}^{\beta} \leq^{p} x\right\} \\
& \cup\left\{\left(x, y_{i}^{\alpha+n}\right): n<\omega, i<i^{*}, x<^{p} y_{i}^{\beta}\right\} .
\end{aligned}
$$

By 2.3.5 we may assume that $i^{*}=\mu$. For $(\bar{x}, h) \in D^{p}=D^{q}$ we let

$$
\begin{array}{r}
\operatorname{dom}\left(d_{\bar{x}, h}^{q}\right)=\operatorname{dom}\left(d_{\bar{x}, h}^{p}\right) \cup\left\{\bar{y} \in\left(t^{q}\right)^{[\mu]}:\left(\exists \bar{y}^{\prime} \in \operatorname{dom}\left(d_{\bar{x}, h}^{p}\right)\right)\left(\bar{x}<\bar{y}<\bar{y}^{\prime}\right)\right. \text { and } \\
\left.(\exists n<\omega)(\forall \varepsilon<\mu)\left(y_{\varepsilon} \in[\lambda \cdot(\alpha+n), \lambda \cdot(\alpha+n+1))\right)\right\},
\end{array}
$$

and let $\alpha_{\bar{x}, h}^{*}=\sup \left(\operatorname{rang}\left(d_{\bar{x}, h}^{p}\right) \cup\left\{\gamma:\left(\exists \bar{y}^{\prime}\right)\left(\left(\bar{y}^{\prime}, \gamma\right) \in \operatorname{dom}\left(e_{\bar{x}, h}^{p}\right)\right)\right\}\right)$. Fix an enumeration $\left\{\bar{y}_{\xi}: \xi<\zeta\right\}$ of $\operatorname{dom}\left(d_{\bar{x}, h}^{q}\right) \backslash \operatorname{dom}\left(d_{\bar{x}, h}^{p}\right)$ such that $\bar{y}_{\xi_{0}}<\bar{y}_{\xi_{1}} \Rightarrow$ $\xi_{0}<\xi_{1}$. We put $d_{\bar{x}, h}^{q}\left(\bar{y}_{\xi}\right)=\alpha_{\bar{x}, h}^{*}+1+\xi$ for $\xi<\zeta$ (and we declare $d_{\bar{x}, h}^{q} \supseteq$ $\left.d_{\bar{x}, h}^{p}\right)$. Next we define $e_{\bar{x}, h}^{q}$ similarly to 2.3.5(4) putting the value 2 whenever possible $\left(\operatorname{so} \operatorname{dom}\left(e_{\bar{x}, h}^{q}\right)=\left\{(\bar{y}, \gamma): \bar{y} \in \operatorname{dom}\left(d_{\bar{x}, h}^{q}\right) \& \gamma<\alpha_{\bar{x}, h}^{*}+1+\zeta\right\}\right)$. Now comes the main point: we have to define functions $f^{q}, c^{q}$ (extending $f^{p}, c^{p}$, respectively) such that clauses $(\mathrm{I})+(\mathrm{J})+(\mathrm{K})$ hold. But it should be clear that each instance of clause (I) in $t^{q}$ can be reduced to an instance of this clause in $t^{p}$ (just look at the definitions of $t^{q}, d_{\bar{x}, h}^{q}, e_{\bar{x}, h}^{q}$ ). Thus what we really have to take care of are instances of $(\mathrm{J})$ and $(\mathrm{K})$. For this we define $c^{q}\left\lceil\left\{y_{i}^{\alpha+n}: i<\mu\right\}\right.$ and $f^{q}\left\lceil\left\{y_{i}^{\alpha+n+1}: i<\mu\right\}\right.$ by induction on $n<\omega$. At the first stage (for $n=0$ ) we let

$$
\begin{array}{r}
P=\left\{(\zeta, \bar{x}, h, \bar{y}, \bar{z}):(\bar{x}, h) \in D^{q} \text { and } \zeta<\mu \text { and } \bar{x}<\bar{y}<\bar{z} \in \operatorname{dom}\left(d_{\bar{x}, h}^{q}\right),\right. \\
\text { and } \left.\bar{z} \subseteq\left\{y_{i}^{\alpha+1}: i<\mu\right\} \text { and } \bar{y} \subseteq\left\{y_{i}^{\alpha}: i<\mu\right\}\right\} .
\end{array}
$$

Take a list $\left\langle\left(X^{\Upsilon}, \zeta^{\Upsilon}, \bar{x}^{\Upsilon}, h^{\Upsilon}, \bar{y}^{\Upsilon}, \bar{z}^{\Upsilon}\right): \Upsilon<\mu\right\rangle$ of

$$
\{(X, \zeta, \bar{x}, h, \bar{y}, \bar{z}): X \in\{J, K\} \&(\zeta, \bar{x}, h, \bar{y}, \bar{z}) \in P\}
$$


in which each 6 -tuple appears $\mu$ times and $\zeta^{\Upsilon} \leq 1+\Upsilon$. Next by induction on $\Upsilon<\mu$ choose a sequence $\left\langle c_{\Upsilon}, f_{\Upsilon}: \Upsilon \leq \mu\right\rangle$ such that:

$(\alpha) c_{\Upsilon}: \operatorname{dom}\left(c_{\Upsilon}\right) \rightarrow \mu, \operatorname{dom}\left(c_{\Upsilon}\right) \subseteq\left\{y_{i}^{\alpha}: i<\mu\right\},\left|\operatorname{dom}\left(c_{\Upsilon}\right)\right|<\aleph_{0}+|\Upsilon|^{+}$,

$(\beta) f_{\Upsilon}: \operatorname{dom}\left(f_{\Upsilon}\right) \rightarrow \lambda, \operatorname{dom}\left(f_{\Upsilon}\right) \subseteq\left\{y_{i}^{\alpha+1}: i<\mu\right\},\left|\operatorname{dom}\left(f_{\Upsilon}\right)\right|<\aleph_{0}+$ $|\Upsilon|^{+}$

$(\gamma)\left\langle c_{\Upsilon}: \Upsilon \leq \mu\right\rangle$ and $\left\langle f_{\Upsilon}: \Upsilon \leq \mu\right\rangle$ are increasing continuous,

$(\delta)$ for each $\Upsilon<\mu$ there is $i^{\Upsilon}<\mu$ divisible by $\zeta^{\Upsilon}$ such that

$$
\begin{aligned}
& \operatorname{rang}\left(\bar{z}^{\Upsilon} \uparrow\left[i^{\Upsilon}, i^{\Upsilon}+\zeta^{\Upsilon}\right)\right) \subseteq \operatorname{dom}\left(f_{\Upsilon+1}\right) \backslash \operatorname{dom}\left(f_{\Upsilon}\right), \\
& \operatorname{rang}\left(\bar{y}^{\Upsilon} \uparrow\left[i^{\Upsilon}, i^{\Upsilon}+\zeta^{\Upsilon}\right)\right) \subseteq \operatorname{dom}\left(c_{\Upsilon+1}\right) \backslash \operatorname{dom}\left(c_{\Upsilon}\right),
\end{aligned}
$$

$(\varepsilon)$ if $X^{\Upsilon}=J$ and $h^{\Upsilon} \in H_{\mu}^{3, *}$ then condition 2.3.2(J) holds for $\bar{x}^{\Upsilon}, h^{\Upsilon}$, $\bar{y}^{\Upsilon}, \bar{z}^{\Upsilon}$ with $i=i^{\Upsilon}$,

$(\zeta)$ if $X^{\Upsilon}=K$ then $c_{\Upsilon+1}\left(\left(\bar{y}^{\Upsilon}\right)_{i} \Upsilon\right)=\zeta^{\Upsilon}$,

(८) $y_{\Upsilon}^{\alpha} \in \operatorname{dom}\left(c_{\Upsilon+1}\right), y_{\Upsilon}^{\alpha+1} \in \operatorname{dom}\left(f_{\Upsilon+1}\right)$.

There are no difficulties with carrying out the construction: the only possible troubles could come from demand $(\varepsilon)$ above. But look at the definition 2.2(14) of $H_{\mu}^{3}$. Taking sufficiently large $\beta<\mu^{+}=\lambda$, the respective sequences $\left\langle\alpha_{\varepsilon}^{0}: \varepsilon\left\langle\zeta^{\Upsilon}\right\rangle,\left\langle\alpha_{\varepsilon}^{1}: \varepsilon<\zeta^{\Upsilon}\right\rangle\right.$ will be good candidates for $c_{\Upsilon+1} \uparrow\left(\bar{y}^{\Upsilon} \uparrow\left[i^{\Upsilon}, i^{\Upsilon}+\zeta^{\Upsilon}\right)\right)$ and $f_{\Upsilon+1} \uparrow\left(\bar{z}^{\Upsilon} \uparrow\left[i^{\Upsilon}, i^{\Upsilon}+\zeta^{\Upsilon}\right)\right)$ in clause $(\varepsilon)$.

The functions $c_{\mu}, f_{\mu}$ will be the respective restrictions $c^{q} \uparrow\left\{y_{i}^{\alpha}: i<\mu\right\}$ and $f^{q}\left\lceil\left\{y_{i}^{\alpha+1}: i<\mu\right\}\right.$. Next, arriving at stage $n+1$ of the definition we repeat the above procedure with no changes. Note that at this stage we know $c^{q} \uparrow\left\{y_{i}^{\alpha+n}: i<\mu\right\}$ and $f^{q} \uparrow\left\{y_{i}^{\alpha+n+1}: i<\mu\right\}$ but they have no influence on defining $c^{q}$ and $f^{q}$ at levels $\alpha+n+1$ and $\alpha+n+2$. $\mathbf{m}_{2.3 .6}$

Claim 2.3.7 (The Amalgamation Property). Assume that $\alpha<\lambda^{+}$is either a successor ordinal or $\operatorname{cf}(\alpha)=\lambda, p, q \in \mathcal{A P}$ and $p\left\lceil\omega \cdot \alpha \leq q \in \mathcal{A P}_{\alpha}\right.$. Then there is $r \in \mathcal{A P}$ such that $p, q \leq r$.

P r o of. First try just the $r$ defined by

$$
\begin{gathered}
w^{r}=w^{p} \cup w^{q}, \quad t^{r}=t^{p} \cup t^{q}, \quad \leq^{r}=\leq^{p} \cup \leq^{q}, \quad D^{r}=D^{p} \cup D^{q}, \\
d_{\bar{x}, h}^{r}= \begin{cases}d_{\bar{x}, h}^{p} & \text { if }(\bar{x}, h) \in D^{p} \backslash D^{q}, \\
d_{\bar{x}, h}^{q} & \text { if }(\bar{x}, h) \in D^{q} \backslash D^{p}, \\
d_{\bar{x}, h}^{p} \cup d_{\bar{x}, h}^{q} & \text { if }(\bar{x}, h) \in D^{q} \cap D^{p},\end{cases}
\end{gathered}
$$

and $e_{\bar{x}, h}^{r} \supseteq e_{\bar{x}, h}^{p} \cup e_{\bar{x}, h}^{q}$ (defined naturally, i.e. with $\operatorname{dom}\left(e_{\bar{x}, h}^{r}\right)$ minimal possible to satisfy demand (F) and value 2 whenever possible), $f^{r}=f^{p} \cup f^{q}$ and $c^{r}=c^{p} \cup c^{q}$. Clearly $p \leq r$ and $q \leq r, w^{r}=w^{p} \cup w^{q}$, but does $r$ belong to $\mathcal{A P}$ ? The things that might go wrong are:

- there is $y \in t^{p} \backslash t^{q}$ which has nothing below it in some levels, or

- $y \wedge z$ is not defined for some $y, z$, or

- the relevant cases of clauses (I)-(K) fail. 
Let $\beta_{0}=\bigcup\left\{\gamma: \omega \cdot \gamma \in w^{p} \cap \omega \cdot \alpha\right\}=\sup (\operatorname{dom}(p) \cap \alpha)$. Note that $\beta_{0} \in$ $\operatorname{dom}(p) \cap \alpha$ (as $\alpha$ is either a successor or of cofinality $\lambda$ ) and

$$
\text { if } w^{q} \subseteq \omega \cdot \beta_{0}+\omega \text { then } r \in \mathcal{A P} \text {. }
$$

So we assume from now on that $w^{q} \not \subset \omega \cdot \beta_{0}+\omega$ (by $(\circledast)$ above). Then necessarily $\beta_{0}+1<\alpha$ (as $\left.w^{q} \subseteq \omega \cdot \alpha\right)$. Without loss of generality $\operatorname{dom}(p) \backslash \alpha \neq$ $\emptyset$ (as if $w^{p} \subseteq \omega \cdot \alpha$ we can let $r=q$ ) and $q\left\lceil\omega \cdot\left(\beta_{0}+1\right)=p\lceil\omega \cdot \alpha\right.$. Let $\alpha^{*}:=\min (\operatorname{dom}(p) \backslash \alpha)$ and $\beta^{*}:=\min \left(\operatorname{dom}(q) \backslash\left(\beta_{0}+1\right)\right)$. By 2.3.6 we may assume that $\beta^{*}=\beta_{0}+1$ (i.e. $\left.\omega \cdot \beta_{0}+\omega \in w^{q}\right)$. Let $\left\{x_{i}^{\omega \cdot \alpha^{*}}: i<i^{*}\right\}$ list $t^{p} \cap\left[\lambda \cdot\left(\omega \cdot \alpha^{*}\right), \lambda \cdot\left(\omega \cdot \alpha^{*}\right)+\lambda\right)$. By 2.3.5(1) (i.e. increasing $q$ only by increasing $t^{q} \cap\left[\lambda \cdot\left(\omega \cdot \beta^{*}\right), \lambda \cdot\left(\left(\omega \cdot \beta^{*}\right)+1\right)\right)$ we may assume that there is a list $\left\{x_{i}^{\omega \cdot \beta^{*}}: i<i^{*}\right\}$ of distinct members of $t^{q} \cap\left[\lambda \cdot\left(\omega \cdot \beta^{*}\right), \lambda \cdot\left(\omega \cdot \beta^{*}\right)+\lambda\right)$ such that $\left(\forall z \in t^{p} \cap t^{q}\right)\left[z<^{p} x_{i}^{\omega \cdot \alpha^{*}} \equiv z<^{q} x_{i}^{\omega \cdot \beta^{*}}\right]$. Let $x_{i}^{\beta} \in[\lambda \cdot \beta, \lambda \cdot \beta+\lambda)$ (for $\beta \in w^{q} \backslash\left(\omega \cdot \beta^{*}+1\right.$ ) and $i<i^{*}$ ) be pairwise distinct and not in $t^{q}$. Now we shall "correct" $r$ to $r^{*}$ :

$$
\begin{aligned}
t^{r^{*}}=t^{r} \cup\left\{x_{i}^{\beta}: \beta \in w^{q} \backslash\left(\omega \cdot \beta^{*}+1\right), i<i^{*}\right\}, \quad w^{r^{*}}=w^{r}, \\
\leq^{r^{*}}=\leq^{r} \cup\left\{\left(x_{i}^{\beta}, x\right): i<i^{*}, x \in t^{p}, x_{i}^{\omega \cdot \alpha^{*}} \leq^{p} x, \beta \in w^{q} \backslash \omega \cdot \beta^{*}\right\} \\
\cup\left\{\left(x, x_{i}^{\beta}\right): i<i^{*}, x \in t^{q}, x \leq^{q} x_{i}^{\omega \cdot \beta^{*}}, \beta \in w^{q} \backslash \omega \cdot \beta^{*}\right\} \\
\cup\left\{\left(x_{i}^{\beta_{0}}, x_{i}^{\beta_{1}}\right): \beta_{0}, \beta_{1} \in w^{q} \backslash \omega \cdot \beta^{*}, \beta_{0} \leq \beta_{1}, i<i^{*}\right\} .
\end{aligned}
$$

Put $D^{r^{*}}=D^{r}$. If $(\bar{x}, h) \in D^{r} \backslash D^{p\lceil\omega \cdot \alpha}$ then we can let $d_{\bar{x}, h}^{r^{*}}=d_{\bar{x}, h}^{r}$, but if $(\bar{x}, h) \in D^{p \nmid \omega \cdot \alpha}$ then we first let

$$
\gamma_{\bar{x}, h}^{*}=\sup \left(\operatorname{rang}\left(d_{\bar{x}, h}^{r}\right) \cup\left\{\gamma:\left(\exists \bar{y}^{\prime}\right)\left((\bar{y}, \gamma) \in \operatorname{dom}\left(e_{\bar{x}, h}^{p}\right) \cup \operatorname{dom}\left(e_{\bar{x}, h}^{q}\right)\right)\right\}\right)
$$

and

$$
\begin{aligned}
\operatorname{dom}\left(d_{\bar{x}, h}^{r^{*}}\right)=\{ & \left\{\bar{y} \in\left(t^{r^{*}}\right)^{[\mu]}: \bar{y} \in \operatorname{dom}\left(d_{\bar{x}, h}^{r}\right)\right. \text { or for some } \\
& \bar{z}=\left\langle x_{\varepsilon(j)}^{\omega \cdot \alpha^{*}}: j<\mu\right\rangle \in\left(t^{p}\right)_{\operatorname{otp}\left(w^{p} \cap \omega \cdot \alpha^{*}\right)}^{[\mu]} \text { and } \beta \in w^{q} \backslash\left(\omega \cdot \beta^{*}+1\right) \\
& \text { we have } \left.\bar{z} \in \operatorname{dom}\left(d_{\bar{x}, h}^{p}\right) \text { and } \bar{y}=\left\langle x_{\varepsilon(j)}^{\beta}: j<\mu\right\rangle\right\} .
\end{aligned}
$$

Choose $d_{\bar{x}, h}^{r^{*}}$ in such a manner that $d_{\bar{x}, h}^{r^{*}} \supseteq d_{\bar{x}, h}^{r}$ and the values $d_{\bar{x}, h}^{r^{*}}(\bar{y})$, if not defined before, are distinct ordinals from $\left(\gamma_{\bar{x}, h}^{*}, \lambda\right)$. Thus, in particular,

$$
d_{\bar{x}, h}^{r^{*}}(\bar{y})=d_{\bar{x}, h}^{r^{*}}(\bar{z}) \& \bar{y} \neq \bar{z} \Rightarrow\{\bar{y}, \bar{z}\} \subseteq \operatorname{dom}\left(d_{\bar{x}, h}^{r}\right) .
$$

Next we define $e_{\bar{x}, h}^{r^{*}}$ extending $e_{\bar{x}, h}^{r}$ to satisfy clause $(\mathrm{F})$ - we put the value 2 whenever possible. [Note that this is the place where the assumption that $\bar{y} \in t_{\bar{x}}^{\langle\mu\rangle}$ in clause (F)(ii), and so the respective assumption in $2.3(2 \mathrm{~b})$, plays a role: the values of $e_{\bar{x}, h}^{q}$ at the level $\omega \cdot \beta^{*}=\omega \cdot \beta_{0}+\omega$ do not interfere with the values of $e_{\bar{x}, h}^{p}$ at the level $\omega \cdot \alpha^{*}$ since $\beta^{*}$ is a successor, not of 
cofinality $\lambda$.$] Now we have to define c^{r^{*}} \supseteq c^{r}$ and $f^{r^{*}} \supseteq f^{r}$, i.e. to define

$$
\begin{aligned}
& c^{r^{*}}\left\lceil\left\{x_{i}^{\beta}: i<i^{*}, \beta \in w^{q} \backslash\left(\omega \cdot \beta^{*}+1\right)\right\},\right. \\
& f^{r^{*}}\left\lceil\left\{x_{i}^{\beta}: i<i^{*}, \beta \in w^{q} \backslash \omega \cdot \beta^{*} \text { is a successor }\right\},\right.
\end{aligned}
$$

such that clauses 2.3.2(G)-(K) hold. This is done as in 2.3.6, but now the clause (I) is "active" too. Of course, the point is that we have $\mu$ commitments, each has " $\mu$ disjoint chances", so we list them in a list of length $\mu$ and inductively we can easily do it (for $\mu$ singular - at place $i$ of the list there may appear only a commitment of "size $\leq|i|+\aleph_{0}$ "). More fully, let

$$
\begin{aligned}
P^{1}=\{ & (\zeta, \bar{x}, h, \bar{y}, \bar{z}):(\bar{x}, h) \in D^{p} \cap D^{q} \text { and } \zeta<\mu \text { and for some } \varepsilon, \\
& (\bar{z}, \varepsilon) \in \operatorname{dom}\left(e_{\bar{x}, h}^{r^{*}}\right) \backslash \operatorname{dom}\left(e_{\bar{x}, h}^{r}\right) \text { and }(\bar{y}, \varepsilon) \in \operatorname{dom}\left(e_{\bar{x}, h}^{q}\right) \text { and } \\
& e_{\bar{x}, h}^{r^{*}}(\bar{z}, \varepsilon) \leq 1 \text { and } e_{\bar{x}, h}^{q}(\bar{y}, \varepsilon) \leq 1 \text { and } \\
& \left.\bar{y} \subseteq\left[\lambda\left(\omega \cdot \beta^{*}+1\right), \lambda\left(\omega \cdot \beta^{*}+1\right)+\lambda\right) \text { and } \bar{z} \subseteq\left\{x_{i}^{\omega \cdot \beta^{*}+1}: i<i^{*}\right\}\right\}
\end{aligned}
$$

Defining $f$ we have to take care of condition (I) for all $(\zeta, \bar{x}, h, \bar{y}, \bar{z}) \in P^{1}$. We also have to take care of conditions $(\mathrm{J}),(\mathrm{K})$ for

$$
\begin{aligned}
P^{2}= & \left\{(\zeta, \bar{x}, h, \bar{y}, \bar{z}):(\bar{x}, h) \in D^{p} \cap D^{q} \text { and } \zeta<\mu\right. \text { and } \\
& \left.\bar{z} \in \operatorname{dom}\left(d_{\bar{x}, h}^{r^{*}}\right), \bar{z} \subseteq\left\{x_{i}^{\gamma+1}: i<i^{*}\right\}, \bar{y}=\bar{z} \mid \gamma, \gamma \in w^{q} \backslash \omega \cdot \beta^{*}\right\} .
\end{aligned}
$$

So we use a list $\left\langle\left(X^{\Upsilon}, \zeta^{\Upsilon}, \bar{x}^{\Upsilon}, h^{\Upsilon}, \bar{y}^{\Upsilon}, \bar{z}^{\Upsilon}\right): \Upsilon<\mu\right\rangle$ of $\{(X, \zeta, \bar{x}, h, \bar{y}, \bar{z}): X \in$ $\{1,2\}$ and $\left.(\zeta, \bar{x}, h, \bar{y}, \bar{z}) \in P^{1} \cup P^{2}\right\}$ in which each 6-tuple appears $\mu$ times and $\zeta^{\Upsilon} \leq 1+\Upsilon$. Let $\left\{x^{\Upsilon}: \Upsilon<\mu\right\}$ list $t^{r^{*}} \backslash t^{r}$. Now we define by induction on $\Upsilon \leq \mu$ functions $c_{\Upsilon}, f_{\Upsilon}$ such that:

$(\alpha) c_{\Upsilon}$ is a function extending $c^{r}$ with $\operatorname{rang}\left(c_{\Upsilon}\right) \subseteq \lambda$,

$(\beta) \operatorname{dom}\left(c_{\Upsilon}\right) \backslash \operatorname{dom}\left(c^{r}\right)$ is a subset of $\left\{x_{i}^{\beta}: i<i^{*}, \beta \in w^{q} \backslash\left(\omega \cdot \beta^{*}+1\right)\right\}$ of cardinality $<\aleph_{0}+|\Upsilon|^{+}$,

$(\gamma) f_{\Upsilon}$ is a function extending $f^{r}$ with $\operatorname{rang}\left(f_{\Upsilon}\right) \subseteq \lambda$,

$(\delta) \operatorname{dom}\left(f_{\Upsilon}\right) \backslash \operatorname{dom}\left(f^{r}\right)$ is a subset of

$$
\left\{x_{i}^{\beta}: i<i^{*}, \beta \text { is a successor ordinal and } \beta \in w^{q} \backslash\left(\omega \cdot \beta^{*}+1\right)\right\}
$$

of cardinality $<\aleph_{0}+|\Upsilon|^{+}$,

$(\varepsilon)$ the sequences $\left\langle c_{\Upsilon}: \Upsilon \leq \mu\right\rangle$ and $\left\langle f_{\Upsilon}: \Upsilon \leq \mu\right\rangle$ are increasing continuous,

$(\zeta)$ for each $\Upsilon$ there is $i^{\Upsilon}<\mu$ divisible by $\zeta^{\Upsilon}$ such that if $X^{\Upsilon}=I$ then $\operatorname{rang}\left(\bar{z}^{\Upsilon} \uparrow\left[i^{\Upsilon}, i^{\Upsilon}+\zeta^{\Upsilon}\right)\right) \subseteq\left[\operatorname{dom}\left(c_{\Upsilon+1}\right) \backslash \operatorname{dom}\left(c_{\Upsilon}\right)\right] \cap\left[\operatorname{dom}\left(f_{\Upsilon+1}\right) \backslash \operatorname{dom}\left(f_{\Upsilon}\right)\right]$, and if $X^{\Upsilon} \in\{J, K\},\left(\zeta^{\Upsilon}, \bar{x}^{\Upsilon}, h^{\Upsilon}, \bar{y}^{\Upsilon}, \bar{z}^{\Upsilon}\right) \in P^{2}$ then

$$
\begin{aligned}
& \operatorname{rang}\left(\bar{z}^{\Upsilon} \uparrow\left[i^{\Upsilon}, i^{\Upsilon}+\zeta^{\Upsilon}\right)\right) \subseteq \operatorname{dom}\left(f_{\Upsilon+1}\right) \backslash \operatorname{dom}\left(f_{\Upsilon}\right), \\
& \operatorname{rang}\left(\bar{y}^{\Upsilon} \uparrow\left[i^{\Upsilon}, i^{\Upsilon}+\zeta^{\Upsilon}\right)\right) \subseteq\left(\operatorname{dom}\left(c_{\Upsilon+1}\right) \backslash \operatorname{dom}\left(c_{\Upsilon}\right)\right) \cup c^{r},
\end{aligned}
$$


(८) if $X^{\Upsilon}=I$ and $\left(\zeta^{\Upsilon}, \bar{x}^{\Upsilon}, h^{\Upsilon}, \bar{y}^{\Upsilon}, \bar{z}^{\Upsilon}\right) \in P^{1}$ then condition 2.3.2(I) holds for $\left(\zeta^{\Upsilon}, \bar{x}^{\Upsilon}, h^{\Upsilon}, \bar{y}^{\Upsilon}, \bar{z}^{\Upsilon}, i^{\Upsilon}\right)$,

$(\kappa)$ if $X^{\Upsilon}=J,\left(\zeta^{\Upsilon}, \bar{x}^{\Upsilon}, h^{\Upsilon}, \bar{y}^{\Upsilon}, \bar{z}^{\Upsilon}\right) \in P^{2}$ and $h^{\Upsilon} \in H_{\mu}^{3, *}$ then condition 2.3.2(J) holds for $\left(\bar{x}^{\Upsilon}, h^{\Upsilon}, \bar{y}^{\Upsilon}, \bar{z}^{\Upsilon}, i^{\Upsilon}\right)$,

$(\lambda)$ if $X^{\Upsilon}=K$ and $\bar{y}^{\Upsilon} \nsubseteq \operatorname{dom}\left(c^{r}\right)$ then $c_{\Upsilon+1}\left(\left(\bar{y}^{\Upsilon}\right)_{i} r\right)=\zeta^{\Upsilon}$,

$(\mu) x^{\Upsilon} \in \operatorname{dom}\left(c_{\Upsilon+1}\right)$ and if $x^{\Upsilon}$ is from a successor level of $t^{r^{*}}$ then $x^{\Upsilon} \in \operatorname{dom}\left(f_{\Upsilon+1}\right)$,

$(\nu) \operatorname{rang}\left(f^{\Upsilon} \uparrow\left\{x_{i}^{\omega \cdot \beta^{*}+1}: i<i^{*}\right\}\right) \cap \operatorname{rang}\left(f^{q}\right)=\emptyset$.

The definition is carried out as in 2.3.6. The new points are clause $(\iota)$ and instances of clause $(\kappa)$ for $\Upsilon$ such that $\bar{y}^{\Upsilon} \subseteq\left\{x_{i}^{\omega \cdot \beta^{*}}: i<i^{*}\right\}$. In the second case a potential trouble could be caused by the fact that the function $c_{\Upsilon}$ is defined on $\bar{y}^{\Upsilon}$ already. But the definition 2.2(14) of $H_{\mu}^{3}$ was exactly what we need to handle this: we may find suitable values for $f_{\Upsilon+1} \uparrow\left(\bar{z} \uparrow\left\lceil i^{\Upsilon}, i^{\Upsilon}, \zeta^{\Upsilon}\right)\right)$. To deal with clause $(\iota)$ note that if $h^{\Upsilon} \in H_{\mu}^{3, *}$ then demand 2.3.2(J) for $q$ provides the needed candidates for values of $f_{\Upsilon+1}$; if $h^{\Upsilon} \in H_{\mu}^{1 \text {,* }}$ then the definition 2.2(12) of $H_{\mu}^{1}$ works.

The functions $c_{\mu}, f_{\mu}$ are as required.

The demands (e)-(h) of 2.1(2) are easy now:

Claim 2.3.8. (1) If a sequence $\left\langle p_{i}: i<\delta\right\rangle \subseteq \mathcal{A P}$ is increasing and $\delta<\lambda$ then it has an upper bound $q \in \mathcal{A P}$ such that $\left.\operatorname{dom}(q)=\operatorname{cl}\left(\bigcup_{i<\delta} \operatorname{dom}\left(p_{i}\right)\right)\right)$.

(2) Assume $\beta<\lambda^{+}, \operatorname{cf}(\beta)=\lambda$ and $\delta<\lambda$. Let $\left\langle p_{i}: i<\delta\right\rangle \subseteq \mathcal{A P}_{\beta+1}$ be an increasing sequence and let $q \in \mathcal{A} \mathcal{P}_{\beta}$ be an upper bound for $\left\langle p_{i} \uparrow \omega \cdot \beta\right.$ : $i<\delta\rangle$. Then the family $\left\{p_{i}: i<\delta\right\} \cup\{q\}$ has an upper bound $r$ such that $r\lceil\omega \cdot \beta \geq q$.

(3) Assume that $\left\langle\beta_{i}: i<\delta\right\rangle \subseteq \lambda^{+}$is strictly increasing, each $\beta_{i}$ is either a successor or has cofinality $\lambda$, and $\delta<\lambda$ is limit. Suppose that $q \in \mathcal{A P}$ and $\left\langle p_{i}: i<\delta\right\rangle \subseteq \mathcal{A P}$ is an increasing sequence such that

$$
(\forall i<\delta)\left(q \uparrow \omega \cdot \beta_{i} \leq p_{i} \in \mathcal{A P}_{\beta_{i}}\right)
$$

Then the family $\left\{p_{i}: i<\delta\right\} \cup\{q\}$ has an upper bound $r \in \mathcal{A P}$ such that $(\forall i<\delta)\left(p_{i} \leq r \uparrow \omega \cdot \beta_{i}\right)$.

(4) Suppose that $\delta_{1}, \delta_{2}<\lambda$ are limit ordinals and $\left\langle\beta_{j}: j<\delta_{2}\right\rangle \subseteq \lambda^{+}$is a strictly increasing sequence of ordinals, each $\beta_{j}$ either a successor or of cofinality $\lambda$. Let

$$
\left\langle p_{i, j}:(i, j) \in\left(\delta_{1}+1\right) \times\left(\delta_{2}+1\right) \backslash\left\{\left(\delta_{1}, \delta_{2}\right)\right\}\right\rangle \subseteq \mathcal{A P}
$$

be such that

$$
p_{i, j} \in \mathcal{A P}_{\beta_{j}}, \quad i \leq i^{\prime} \Rightarrow p_{i, j} \leq p_{i^{\prime}, j}, \quad j \leq j^{\prime} \Rightarrow p_{i, j} \leq p_{i, j^{\prime}} \uparrow \omega \cdot \beta_{j} .
$$

Then the family $\left\{p_{i, j}:(i, j) \in\left(\delta_{1}+1\right) \times\left(\delta_{2}+1\right) \backslash\left\{\left(\delta_{1}, \delta_{2}\right)\right\}\right\}$ has an upper bound $r \in \mathcal{A P}$ such that $\left(\forall j<\delta_{2}\right)\left(r\left\lceil\omega \cdot \beta_{j}=p_{\delta_{1}, j}\right)\right.$. 
P r o of. (1) The first try may be to take the natural union of the sequence $\left\langle p_{i}: i<\delta\right\rangle$. However, it may happen that we will not get a legal approximation, as $\bigcup_{i<\delta} \operatorname{dom}\left(p_{i}\right)$ does not have to be closed. But we may take its closure $\operatorname{cl}\left(\bigcup_{i<\delta} \operatorname{dom}\left(p_{i}\right)\right)$ and apply a procedure similar to the one described in 2.3.6 (successively at each element of $\operatorname{cl}\left(\bigcup_{i<\delta} \operatorname{dom}\left(p_{i}\right)\right) \backslash \bigcup_{i<\delta} \operatorname{dom}\left(p_{i}\right)$ ) and construct the required $q$.

(2)-(4) Similarly to (1) above plus the proof of 2.3.7. $\mathbf{m}_{2.3 .8}$

Now we apply [Sh 405, Appendix]: we find a "sufficiently generic" $G \subseteq$ $\mathcal{A P}$ which gives the $T, c, d$ we need (remember 2.3.5):

$$
T=\left\{\eta_{\varepsilon}: \varepsilon \in t^{p} \text { for some } p \in G\right\}
$$

where for $\varepsilon \in[\lambda \alpha, \lambda \alpha+\lambda)$ we define $\eta_{\varepsilon} \in{ }^{\alpha} \lambda$ by $\gamma=\eta_{\varepsilon}(\beta)$ if and only if

$$
(\exists p \in G)\left(\exists \varepsilon^{\prime} \in[\lambda \cdot(\beta+1), \lambda \cdot(\beta+1)+\lambda)\right)\left(t^{p} \models \text { " } \varepsilon^{\prime}<\varepsilon \text { " \& } f^{p}\left(\varepsilon^{\prime}\right)=\gamma\right) .
$$

This finishes the proof. $\mathbf{m}_{2.3}$

Remark 2.4. (1) Theorem 2.3 is close to [Sh 50], which is a strengthening of the construction of special Aronszajn trees. There essentially we replace $(\gamma)+(\delta)$ by

$(\gamma)^{\prime} y_{i}\left(\operatorname{lev}\left(t_{i}\right)\right), z_{i}\left(\operatorname{lev}\left(t_{i}\right)\right)$ do not depend on $i$.

(2) By the proof of 2.3(2), $T$ is $\lambda$-complete.

(3) We may add to 2.3(2):

(c) $T$ is special, i.e. there is a function $d: \bigcup_{\alpha} T_{\alpha} \rightarrow \lambda$ such that

$$
d(\eta)=d(\rho) \Rightarrow \neg\left[\eta<_{T} \rho\right] .
$$

[Just in the definition of $p \in \mathcal{A P}$ (see 2.3.2) add such $d^{p}: t^{p} \rightarrow \lambda$.]

(4) The reader may wonder why we need the condition " $h(\bar{x})$ has $\mu$ pairwise disjoint members". The point is that when we amalgamate $p$ and $q$ when $p\left\lceil\alpha \leq q\right.$, it may happen that $p$ gives information on levels $\alpha_{n}<\alpha_{n+1}$ (for $n<\omega$ ), $\beta=\bigcup_{n<\omega} \alpha_{n}<\alpha, q$ gives information on the level $\beta$, and when amalgamating the function $f^{q}$ gives information on $f$ on this level and $f$ is supposed to be one-to-one on every $\operatorname{succ}_{T}(\eta)$. So considering $\bar{x} \in\left(t^{q}\right)_{\beta+1}$, $\bar{y} \in\left(t^{p}\right)_{\alpha}$, when we try to define $f\lceil\operatorname{rang}(\bar{y} \uparrow(\beta+1))$, some values are excluded.

Theorem 2.5. Assume that $\lambda=\mu^{+}=2^{\mu}$. Then there is a forcing notion $\mathbb{P}$ which is $(<\lambda)$-complete of size $\lambda^{+}$and satisfies the $\lambda^{+}-c c$ (so it preserves cardinalities, cofinalities and cardinal arithmetic) and such that in $\mathbf{V}^{\mathbb{P}}$ :

(1) There is a $\mu$-entangled linear order $\mathcal{I}$ of cardinality $\lambda^{+}$and density $\lambda$.

(2) Let $\sigma \leq \mu$ be a regular cardinal. There exist linear orders $\mathcal{I}^{\prime}, \mathcal{I}^{\prime \prime}$ of cardinality $\lambda^{+}$such that for any uniform ultrafilter $D$ on $\sigma$ the linear orders $\left(\mathcal{I}^{\prime}\right)^{\sigma} / D$ and $\left(\mathcal{I}^{\prime \prime}\right)^{\sigma} / D$ have isomorphic subsets of cardinality $\lambda^{+}$, but $\mathcal{I}^{\prime}+\mathcal{I}^{\prime \prime}$ 
is $\mu$-entangled. Hence there is a Boolean algebra $B$ which is $\lambda^{+}$-narrow but $B^{\sigma} / D$ is not $\lambda^{+}$-narrow for any uniform ultrafilter $D$ on $\sigma$.

(3) There are a set $R \subseteq{ }^{\lambda} \lambda$ with $|R|=\lambda^{+}$and functions $c, \bar{d}$ such that, letting $T^{+}=\left({ }^{\lambda>} \lambda \cup R, \triangleleft\right)(\triangleleft i$ is being initial segment $)$, we have:

(a) $c$ is a function from ${ }^{\lambda>} \lambda$ to $\lambda$,

(b) $R=\left\{\eta_{\alpha}: \alpha<\lambda^{+}\right\}$(with no repetition), $<_{R}=\left\{\left(\eta_{\alpha}, \eta_{\beta}\right)\right.$ : $\alpha<\beta\} ;$ define

$$
R^{*}=\left\{\left\langle\eta_{\alpha_{i}}: i<\mu\right\rangle: \alpha_{i}<\lambda^{+},\left\langle\alpha_{i}: i<\lambda^{+}\right\rangle \text {is increasing }\right\},
$$

(c) for every $\bar{x} \in T_{<\lambda}^{[\mu]}, \zeta<\mu$, and $h \in{ }^{\zeta}(\lambda \times \lambda \times \lambda), d_{\bar{x}, h}$ is a function from $\left\{\bar{y} \in R^{*}: \bar{x}<\bar{y}\right\}$ to $\lambda$ such that

$$
\begin{aligned}
& {\left[d_{\bar{x}, h}(\bar{y})=d_{\bar{x}, h}(\bar{z}) \& \bar{y}, \bar{z} \in T_{\overline{\bar{z}}}^{[\mu]} \text { are distinct }\right]} \\
& \quad \Rightarrow \sup \left\{\alpha: \eta_{\alpha} \text { appears in } \bar{y}\right\} \neq \sup \left\{\alpha: \eta_{\alpha} \text { appears in } \bar{z}\right\}
\end{aligned}
$$

and for some $\bar{t} \in T_{\bar{x}}^{[\mu]} \cap T_{<\lambda}^{[\mu]}$ and $i^{*}<\mu$ we have:

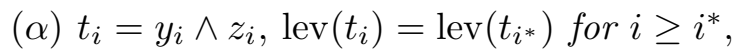

$(\beta)(\forall \varepsilon<\mu)\left(\exists^{\mu} i<\mu\right)\left(c\left(t_{i}\right)=\varepsilon\right)$,

$(\gamma)$ for $\mu$ ordinals $i<\mu$ divisible by $\zeta$, either

(i) there are $\xi_{0}<\xi_{1}<\lambda$ such that

$$
\begin{aligned}
& (\forall \varepsilon<\zeta)\left(\zeta \cdot \xi_{0} \leq y_{i+\varepsilon}(\operatorname{lev}(\bar{t}))<\zeta \cdot \xi_{1} \leq z_{i+\varepsilon}(\operatorname{lev}(\bar{t}))\right) \text { and } \\
& h=\left\langle\left( c\left(t_{i+\varepsilon}\right), y_{i+\varepsilon}(\operatorname{lev}(\bar{t}))-\zeta \cdot \xi_{0},\right.\right. \\
& \left.\left.z_{i+\varepsilon}(\operatorname{lev}(\bar{t}))-\zeta \cdot \xi_{1}\right): \varepsilon<\zeta\right\rangle, \quad \text { or }
\end{aligned}
$$

(ii) a symmetrical requirement interchanging $\bar{y}$ and $\bar{z}$.

Proof. (1) We apply 2.5(3): let $R, c, \bar{d}$ be as there (of course we are in the universe $\mathbf{V}^{\mathbb{P}}$ all the time). We define the order $<_{\mathcal{I}}$ on $\mathcal{I}=R$ by

$$
\begin{aligned}
y<_{\mathcal{I}} z \quad \text { if and only if } & \text { either } c(y \wedge z)=0 \text { and } y(\alpha)<z(\alpha) \\
& \text { or } c(y \wedge z) \neq 0 \text { and } y(\alpha)>z(\alpha),
\end{aligned}
$$

where $\alpha=\operatorname{lev}(y \wedge z)$. Clearly $<_{\mathcal{I}}$ is a linear order of density $\lambda$, and $|\mathcal{I}|=\lambda^{+}$. To show that it is $\mu$-entangled suppose that $y_{\varepsilon}^{\alpha} \in R$ (for $\alpha<\lambda^{+}$and $\varepsilon<\varepsilon(*)<\mu$ ) are pairwise distinct and $u \subseteq \varepsilon(*)$. Let $y_{\varepsilon}^{\alpha}=\eta_{\beta(\alpha, \varepsilon)}$ (for $\alpha<\lambda^{+}$and $\left.\varepsilon<\varepsilon(*)\right)$. We may assume that the truth value of " $\beta\left(\alpha, \varepsilon_{1}\right)<$ $\beta\left(\alpha, \varepsilon_{2}\right)$ " does not depend on $\alpha<\lambda^{+}$. For simplicity, we may assume that for each $\alpha<\lambda^{+}, \varepsilon<\varepsilon^{\prime}<\varepsilon(*)$ implies $y_{\varepsilon}^{\alpha}<_{R} y_{\varepsilon^{\prime}}^{\alpha}$. Finally, without loss of generality we may assume that if $\alpha<\alpha^{\prime}<\lambda^{+}$and $\varepsilon, \varepsilon^{\prime}<\varepsilon(*)$ then $y_{\varepsilon}^{\alpha}<_{R} y_{\varepsilon^{\prime}}^{\alpha^{\prime}}$ (i.e. $\left.\beta(\alpha, \varepsilon)<\beta\left(\alpha^{\prime}, \varepsilon^{\prime}\right)\right)$. For $\varepsilon<\varepsilon(*), i<\mu$ and $\alpha<\lambda^{+}$ let $z_{i \cdot \varepsilon(*)+\varepsilon}^{\alpha}=y_{\varepsilon}^{\alpha \cdot \mu+i \cdot \varepsilon(*)}$ and $\bar{z}^{\alpha}=\left\langle z_{i}^{\alpha}: i<\mu\right\rangle$. Clearly each $\bar{z}^{\alpha}$ is in $R^{*}$. Now for $\alpha<\lambda^{+}$choose $\xi(\alpha)<\lambda$ such that $z_{i}^{\alpha}\lceil\xi(\alpha)$ (for $i<\mu$ ) are pairwise distinct. Without loss of generality we may assume that $\xi(\alpha)=\xi$ 
for $\alpha<\lambda^{+}$. There are $\lambda^{\mu}=\lambda$ possibilities for $\left\langle z_{i}^{\alpha}\lceil\xi: i<\mu\rangle\right.$, so we may assume that for all $\alpha<\lambda^{+}$,

$$
\left\langle z_{i}^{\alpha} \mid \xi: i<\mu\right\rangle=\left\langle x_{i}: i<\mu\right\rangle=\bar{x} \in T_{\xi}^{[\mu]} .
$$

Let $h:=\left\langle\left(0, l_{\varepsilon}^{u}, 1-l_{\varepsilon}^{u}\right): \varepsilon<\varepsilon(*)\right\rangle \in{ }^{\varepsilon(*)}(\lambda \times \lambda \times \lambda)$, where $l_{\varepsilon}^{u}$ is 0 if $\varepsilon \in u$ and 1 otherwise. For some distinct $\alpha_{1}, \alpha_{2}<\lambda^{+}$we have $d_{\bar{x}, h}\left(\bar{z}^{\alpha_{1}}\right)=d_{\bar{x}, h}\left(\bar{z}^{\alpha_{2}}\right)$. By the properties of $d_{\bar{x}, h}$, possibly interchanging $\alpha_{1}, \alpha_{2}$, we find $i<\mu$, ordinals $\xi_{0}<\xi_{1}<\lambda$ and $\bar{t} \in T_{\bar{x}}^{[\mu]}$ such that

$$
\begin{gathered}
(\forall \varepsilon<\varepsilon(*))\left(z_{i \cdot \varepsilon(*)+\varepsilon}^{\alpha_{1}} \wedge z_{i \cdot \varepsilon(*)+\varepsilon}^{\alpha_{2}}=t_{i \cdot \varepsilon(*)+\varepsilon}\right), \\
(\forall \varepsilon<\varepsilon(*))\left(\varepsilon(*) \cdot \xi_{0} \leq z_{i \cdot \varepsilon(*)+\varepsilon}^{\alpha_{1}}(\beta)<\varepsilon(*) \cdot \xi_{1} \leq z_{i \cdot \varepsilon(*)+\varepsilon}^{\alpha_{2}}(\beta),\right. \\
h=\left\langle\left(c\left(t_{i \cdot \varepsilon(*)+\varepsilon}\right), z_{i \cdot \varepsilon(*)+\varepsilon}^{\alpha_{1}}(\beta)-\varepsilon(*) \cdot \xi_{0}, z_{i \cdot \varepsilon(*)+\varepsilon}^{\alpha_{2}}(\beta)-\varepsilon(*) \cdot \xi_{1}\right): \varepsilon<\varepsilon(*)\right\rangle,
\end{gathered}
$$

where $\beta=\operatorname{lev}(\bar{t})$. Then $\alpha_{1} \cdot \mu+i \cdot \varepsilon(*) \neq \alpha_{2} \cdot \mu+i \cdot \varepsilon(*)$ (for $i<\mu$ ) and

$$
(\forall \varepsilon<\varepsilon(*))\left(y_{\varepsilon}^{\alpha_{1} \cdot \mu+i \cdot \varepsilon(*)}<_{\mathcal{I}} y_{\varepsilon}^{\alpha_{2} \cdot \mu+i \cdot \varepsilon(*)} \text { iff } \varepsilon \in u\right)
$$

(by the definition of $<_{\mathcal{I}}$ and the choice of $h$ ), so using 1.2(7) we are done.

(2) As in (1) above, we work in $\mathbf{V}^{\mathbb{P}}$ and we use 2.5(3). Suppose $\sigma \leq \mu$. For a set $A \subseteq \lambda$ we define the order $<_{A}$ on $R$ by

$$
\begin{aligned}
y<_{A} z \quad \text { if and only if } & \text { either } c(y \wedge z) \in A \text { and } y(\alpha)<z(\alpha) \\
& \text { or } c(y \wedge z) \notin A \text { and } z(\alpha)<y(\alpha),
\end{aligned}
$$

where $\alpha=\operatorname{lev}(y \wedge z)$. [Note that the order $<_{\mathcal{I}}$ from part (1) above is just $<_{\{0\}}$.] Clearly $<_{A}$ is a linear order. As in the proof of 2.5(1) one can show that it is $\mu$-entangled. As $\sigma^{<\sigma} \leq \lambda$ we may choose sets $A_{i} \subseteq \lambda$ for $i \leq \sigma$ such that:

(i) for each $\alpha<\lambda$ the set $\left\{i<\sigma: \alpha \in A_{i} \equiv \alpha \notin A_{\sigma}\right\}$ has cardinality $<\sigma$, that

(ii) if $v \subseteq \sigma$ and $|v|<\sigma, h: v \cup\{\sigma\} \rightarrow\{0,1\}$ then there is $\alpha \in \lambda$ such

$$
(\forall i \in v \cup\{\sigma\})\left(\alpha \in A_{i} \Leftrightarrow h(i)=1\right) .
$$

For $i \leq \sigma$ let $\mathcal{I}_{i}=\left(R,<_{A_{i}}\right)$. Put $\mathcal{I}^{\prime}=\sum_{i<\sigma} \mathcal{I}_{i}, \mathcal{I}^{\prime \prime}=\mathcal{I}_{\sigma}, \mathcal{I}=\mathcal{I}^{\prime}+\mathcal{I}^{\prime \prime}=$ $\sum_{i \leq \sigma} \mathcal{I}_{i}$. So it is notationally clearer to let $\mathcal{I}_{i}=\left(\{i\} \times R,<_{i}\right)$ with $\left(i, y_{1}\right)<_{i}$ $\left(i, y_{2}\right)$ iff $y_{1}<_{A_{i}} y_{2}$, and $\mathcal{I}=\left((\sigma+1) \times R,<^{*}\right)$ with $\left(i_{1}, y_{1}\right)<^{*}\left(i_{2}, y_{2}\right)$ iff $i_{1}<i_{2}$ or $\left(i_{1}=i_{2} \& y_{1}<A_{i_{1}} y_{2}\right)$.

Claim 2.5.1. If $y_{0}, y_{1} \in R$ and $y_{0}<_{A_{\sigma}} y_{1}$ then the set $\left\{i<\sigma: y_{0}<_{A_{i}} y_{1}\right\}$ is co-bounded.

Proof. Let $t=y_{0} \wedge y_{1}$ and $y_{0}<_{A_{\sigma}} y_{1}$. The set

$$
u:=\left\{i<\sigma: c(t) \in A_{i} \equiv c(t) \in A_{\sigma}\right\}
$$


satisfies $u \subseteq \sigma \&|\sigma \backslash u|<\sigma$. If $c(t) \in A_{\sigma}$ then $y_{0}(\operatorname{lev}(t))<y_{1}(\operatorname{lev}(t))$. Hence $y_{0}<_{A_{i}} y_{1}$ for $i \in u$. If $c(t) \notin A_{\sigma}$ then $y_{0}(\operatorname{lev}(t))>y_{1}(\operatorname{lev}(t))$ and $y_{1}<_{A_{i}} y_{0}$ for $i \in u$. $\mathbf{m}_{2.5 .1}$

Let $\pi: \mathcal{I}^{\prime \prime} \rightarrow \prod_{i<\sigma} \mathcal{I}^{\prime}$ be such that for $y \in \mathcal{I}_{\sigma}, \pi(y)(i)$ is the element of $\mathcal{I}_{i}$ that corresponds to $y$; recall that all orders $\mathcal{I}_{i}$ are defined on $R$. Now, if $D$ is a uniform ultrafilter on $\sigma$ then $\pi / D: \mathcal{I}^{\prime \prime} \rightarrow\left(\mathcal{I}^{\prime}\right)^{\sigma} / D$ is an embedding. Thus both $\left(\mathcal{I}^{\prime}\right)^{\sigma} / D$ and $\left(\mathcal{I}^{\prime \prime}\right)^{\sigma} / D$ contain a copy of $\mathcal{I}^{\prime \prime}$. Now we will finish by the following claim.

Claim 2.5.2. The linear order $\mathcal{I}=\mathcal{I}^{\prime}+\mathcal{I}^{\prime \prime}$ is $\mu$-entangled.

Proof. Suppose that $\varepsilon(*)<\sigma$ and $\left(j_{\varepsilon}^{\alpha}, y_{\varepsilon}^{\alpha}\right) \in \mathcal{I}$ for $\alpha<\lambda^{+}, \varepsilon<\varepsilon(*)$ are pairwise distinct and $u \subseteq \varepsilon(*)$. As $2^{\sigma} \leq \lambda$, without loss of generality $j_{\varepsilon}^{\alpha}=j_{\varepsilon}$. Let $\left\{z_{\zeta}^{\alpha}: \zeta<\zeta_{\alpha}\right\}$ be an enumeration of $Y_{\alpha}:=\left\{y_{\varepsilon}^{\alpha}: \varepsilon<\varepsilon(*)\right\}$ and let $\left\{i_{\xi}: \xi<\xi^{*}\right\}$ enumerate $v:=\left\{j_{\varepsilon}: \varepsilon<\varepsilon(*)\right\}$. We may assume that the sequences $\left\langle z_{\zeta}^{\alpha}: \zeta<\zeta_{\alpha}\right\rangle$ are $<_{R}$-increasing and pairwise disjoint (for $\alpha<\lambda^{+}$) as each $z$ may appear in at most $\varepsilon(*)$ of them. Moreover, we may assume that $\left\{\left(\zeta_{\alpha}, \varepsilon, \zeta, \xi\right):\left(j_{\varepsilon}, y_{\varepsilon}^{\alpha}\right)=\left(i_{\xi}, z_{\zeta}^{\alpha}\right)\right\}$ does not depend on $\alpha$, so $\zeta_{\alpha}=\zeta^{*}$ and by enlarging and renaming instead of $\left\langle\left(j_{\varepsilon}^{\alpha}, y_{\varepsilon}^{\alpha}\right): \varepsilon<\varepsilon(*)\right\rangle$ we have $\left\langle\left(i_{\xi}, z_{\zeta}^{\alpha}\right): \zeta<\zeta^{*}, \xi<\xi^{*}\right\rangle$ and so $u \subseteq \zeta^{*} \times \xi^{*}$. Now, for each $\zeta<\zeta^{*}$ we choose $c[\zeta]<\lambda$ such that

$$
\left(\forall \xi<\xi^{*}\right)\left[c[\zeta] \in A_{i_{\xi}} \Leftrightarrow(\xi, \zeta) \in u\right]
$$

and we proceed as in earlier cases (considering $h=\left\langle(c[\zeta], 0,1): \zeta<\zeta^{*}\right\rangle \in$ $\left.\zeta^{*}(\lambda \times \lambda \times \lambda)\right)\left({ }^{4}\right) \cdot \mathbf{m}_{2.5 .2}$

(3) The definition of the forcing notion $\mathbb{P}$ is somewhat similar to that of the approximations in 2.3(2).

Definition 2.5.3. A condition in $\mathbb{P}$ is a tuple $p=\langle t, \delta, w, \leq, D, \bar{d}, \bar{e}, c\rangle$ (we may write $t^{p}, w^{p}$, etc.) such that:

(A) $w \subseteq \lambda^{+}$is a set of cardinality $<\lambda$ and $\delta$ is a limit ordinal $<\lambda$; let $w^{[\mu]}$ be the family of all increasing sequences $\bar{y} \subseteq w$ of length $\mu$,

(B) $t$ is a non-empty subset of $\delta \geq \lambda$ of cardinality $<\lambda$, closed under initial segments,

(C) $\leq$ is such that $(t \cup w, \leq)$ is a normal tree, $\leq^{p}\lceil t$ is $\triangleleft$, and for $\alpha \in w$, $b_{\alpha}^{p}:=\bigcup\left\{\nu \in t: \nu \leq^{p} \alpha\right\} \in t \cap^{\delta} \lambda$, and $\alpha \neq \beta \Rightarrow b_{\alpha}^{p} \neq b_{\beta}^{p}$,

(D) $c$ is a function from $t$ to $\lambda$,

(E) $D$ is a set of $<\lambda$ pairs $(\bar{x}, h)$ such that $\bar{x} \in t^{[\mu]}$ and $h \in \bigcup_{\zeta<\mu} \zeta(\lambda \times$ $\lambda \times \lambda)$

(F) $\bar{d}=\left\langle d_{\bar{x}, h}:(\bar{x}, h) \in D\right\rangle$, where each $d_{\bar{x}, h}$ is a partial function from $w^{[\mu]}$ to $\delta^{p}$ with domain of cardinality $<\lambda$,

$\left(^{4}\right)$ Alternatively, let $R$ be the disjoint union of $R_{i}(i \leq \sigma)$ and use $\mathcal{I}_{i}^{*}=\mathcal{I}_{i} \uparrow\left(\{i\} \times R_{i}\right)$. 
(G) $\bar{e}=\left\langle e_{\bar{x}, h}:(\bar{x}, h) \in D\right\rangle$, where each $e_{\bar{x}, h}$ is a partial function from $t_{\bar{x}}^{[\mu]} \times \delta^{p}$ to $\{0,2\}$ such that for $(\bar{x}, h) \in D$ :

(i) $\operatorname{dom}\left(e_{\bar{x}, h}\right) \supseteq\left\{(\bar{y}, \gamma)\right.$ : for some $\bar{z}_{0}, \bar{z}_{1} \in \operatorname{dom}\left(d_{\bar{x}, h}\right)$ we have $\bar{x}<$ $\bar{y} \leq \bar{z}_{0}$ and $\left.\left.\gamma=d_{\bar{x}, h}\left(\bar{z}_{1}\right)\right)\right\}$,

(ii) if $\bar{y} \in t_{\bar{x}}^{[\mu]}, \bar{x}<_{t} \bar{y}<_{t} \bar{z}$ and $e_{\bar{x}, h}(\bar{z}, \beta)$ is defined then $e_{\bar{x}, h}(\bar{y}, \beta)$ is defined and $e_{\bar{x}, h}(\bar{y}, \beta) \leq e_{\bar{x}, h}(\bar{z}, \beta)$,

(iii) if $\bar{z} \in \operatorname{dom}\left(d_{\bar{x}, h}\right)$ and $\bar{x}<\bar{y}<\bar{z}$ then $e_{\bar{x}, h}\left(\bar{y}, d_{\bar{x}, h}(\bar{z})\right)=0$,

and letting $h=\left\langle\left(\alpha_{\varepsilon}^{0}, \alpha_{\varepsilon}^{1}, \alpha_{\varepsilon}^{2}\right): \varepsilon<\zeta\right\rangle$ we have

(iv) if $(\bar{y}, \alpha) \in \operatorname{dom}\left(e_{\bar{x}, h}\right), \operatorname{lev}(\bar{y})=\delta^{p}$ and $e_{\bar{x}, h}(\bar{y}, \alpha)=0$ then

$$
(\forall \varepsilon<\mu)\left(\exists^{\mu} i<\mu\right)\left(c\left(u_{i}\right)=\varepsilon\right)
$$

and for $\mu$ ordinals $i<\mu$ divisible by $\zeta$, for some $\xi<\lambda$,

$$
(\forall \varepsilon<\zeta)\left(c\left(y_{i+\varepsilon}\right)=\alpha_{\varepsilon}^{0}\right),
$$

(v) if $\bar{x}<\bar{y}^{0}<\bar{y}^{1}, \alpha \leq \operatorname{lev}\left(\bar{y}^{0}\right)+1=\operatorname{lev}\left(\bar{y}^{1}\right)<\delta^{p}$ and $e_{\bar{x}, h}\left(\bar{y}^{1}, \alpha\right)=$ 0 , then for $\mu$ ordinals $i<\mu$ divisible by $\zeta$, for some $\xi<\lambda$,

$$
(\forall \varepsilon<\zeta)\left(c\left(y_{i+\varepsilon}^{0}\right)=\alpha_{\varepsilon}^{0} \& \zeta \cdot \xi+\alpha_{\varepsilon}^{1}=y_{i+\varepsilon}^{1}\left(\operatorname{lev}\left(\bar{y}^{0}\right)\right)\right)
$$

$(\mathrm{H})$ if $e_{\bar{x}, h}(\bar{y}, \alpha)=e_{\bar{x}, h}(\bar{z}, \alpha)=0, \neg\left[\bar{y} \leq_{t} \bar{z}\right]$ and $\neg\left[\bar{z} \leq_{t} \bar{y}\right]$ then clauses $(\alpha),(\beta),(\gamma)$ of $2.5(4 \mathrm{c})$ hold.

$\mathbb{P}$ is equipped with the natural partial order: $p \leq q$ if and only if $t^{p} \subseteq t^{q}$, $\delta^{p} \leq \delta^{q}, w^{p} \subseteq w^{q}, c^{p} \subseteq c^{q}, D^{p} \subseteq D^{q}$, and

$$
(\bar{x}, h) \in D^{p} \Rightarrow d_{\bar{x}, h}^{p} \subseteq d_{\bar{x}, h}^{q} \& e_{\bar{x}, h}^{p} \subseteq e_{\bar{x}, h}^{q} .
$$

For a condition $p \in \mathbb{P}$ and an ordinal $\alpha<\lambda^{+}$we define $q=p\lceil\alpha$ by:

- $\delta^{q}=\delta^{p}, t^{q}=t^{p}, w^{q}=w^{p} \cap \alpha, \leq^{q}=\leq^{p}\left\lceil t^{q}, D^{q}=D^{p}, c^{q}=c^{p}\right.$,

- if $(\bar{x}, h) \in D^{q}$ then $d_{\bar{x}, h}^{q}=d_{\bar{x}, h}^{p}\left\lceil\left(w^{q}\right)^{[\mu]}\right.$ and $e_{\bar{x}, h}^{q}=e_{\bar{x}, h}^{p}\left\lceil\left(\left(t^{q}\right)_{\bar{x}}^{[\mu]} \times \delta^{q}\right)\right.$.

Observation 2.5.4. If $p \in \mathbb{P}$ and $\alpha<\lambda^{+}$is either a successor or of cofinality $\lambda$ then $p\lceil\alpha \in \mathcal{A P}$ is the unique maximal condition such that $p\lceil\alpha \leq$ $p$ and $w^{p \nmid \alpha}=w^{p} \cap \alpha$.

Claim 2.5.5 (Density Observation). Assume $p \in \mathbb{P}$.

(1) Suppose $\eta \in^{\lambda>\lambda}$. Then there is $q \in \mathbb{P}$ such that

$$
p \leq q, \quad \eta \in t^{q}, \quad w^{p}=w^{q}, \quad D^{p}=D^{q}, \quad \bar{d}^{q}=\bar{d}^{p} .
$$

(2) For each $\beta \in \lambda^{+} \backslash w^{p}$ there is $q \in \mathbb{P}$ such that $p \leq q$ and $\beta \in w^{q}$.

(3) If $\bar{x} \in\left(t^{p}, \leq^{p}\right)^{[\mu]}, \zeta<\mu$ and $h \in \zeta(\lambda \times \lambda \times \lambda)$ then there is $q \in \mathbb{P}$ such that $p \leq q$ and $D^{q}=D^{p} \cup\{(\bar{x}, h)\}$.

(4) If $(\bar{x}, h) \in D^{p}$ and $\bar{x}<\bar{y} \in\left(w^{p}\right)^{[\mu]}$ then there is $q \in \mathcal{A P}$ with $p \leq q$ such that $w^{q}=w^{p}$ and $\bar{y} \in \operatorname{dom}\left(d_{\bar{x}, h}\right)$. 
Proof. (1) We may assume that $\left|t^{p} \cap \delta^{p} \lambda\right|=\mu$, as otherwise it is even easier. So let $\left\langle\nu_{\beta}: \beta<\mu\right\rangle$ enumerate $t^{p} \cap{ }^{\delta^{p}} \lambda$. Put $\delta^{q}=\max \left\{\delta^{p}+\omega, \lg (\eta)+\right.$ $\omega\}$ and fix $\varrho \in \delta^{q} \lambda$ such that $\eta \triangleleft \varrho$. Next, by induction on $\gamma \in\left(\delta^{p}, \delta^{q}\right]$ define sequences $\nu_{\beta}^{\gamma}$ for $\beta<\mu$ and functions $c_{\gamma}$ such that:

(a) $c_{\gamma}:\left\{\nu_{\beta}^{\gamma}: \beta<\mu\right\} \rightarrow \lambda, \nu_{\beta}^{\gamma} \in{ }^{\gamma} \lambda$.

(b) $\delta^{p}<\gamma_{0}<\gamma_{1} \leq \delta^{q} \Rightarrow \nu_{\beta} \triangleleft \nu_{\beta}^{\gamma_{0}} \triangleleft \nu_{\beta}^{\gamma_{1}}$.

(c) Suppose that $(\bar{x}, h) \in D^{p}, h=\left\langle\left(\alpha_{\varepsilon}^{0}, \alpha_{\varepsilon}^{1}, \alpha_{\varepsilon}^{2}\right): \varepsilon<\zeta\right\rangle \in{ }^{\zeta}(\lambda \times \lambda \times \lambda)$, $\bar{x}<\bar{y}=\left\langle\nu_{\varepsilon(i)}: i<\mu\right\rangle \subseteq t^{p} \cap{ }^{\delta^{p}} \lambda,(\bar{y}, \alpha) \in \operatorname{dom}\left(e_{\bar{x}, h}^{p}\right)$ and $e_{\bar{x}, h}^{p}(\bar{y}, \alpha)=0$. Then

( $\alpha$ ) for $\mu$ ordinals $i<\mu$ divisible by $\zeta$, for some $\xi<\lambda$,

$$
(\forall j<\zeta)\left(c^{p}\left(\nu_{\varepsilon(i+j)}\right)=\alpha_{j}^{0} \& \nu_{\varepsilon(i+j)}^{\delta^{p}+1}\left(\delta^{p}\right)=\zeta \cdot \xi+\alpha_{j}^{1}\right),
$$

$(\beta)$ for each $\gamma \in\left(\delta^{p}, \delta^{q}\right)$ we have

$$
(\forall \xi<\mu)\left(\exists^{\mu} i<\mu\right)\left(c_{\gamma}\left(\nu_{\varepsilon(i)}^{\gamma}\right)=\xi\right)
$$

and for $\mu$ ordinals $i<\mu$ divisible by $\zeta$ there is $\xi<\lambda$ such that

$$
(\forall j<\zeta)\left(c_{\gamma}\left(\nu_{\varepsilon(i+j)}^{\gamma}\right)=\alpha_{j}^{0} \& \nu_{\varepsilon(i+j)}^{\gamma+1}=\zeta \cdot \xi+\alpha_{j}^{1}\right),
$$

$(\gamma)(\forall \xi<\mu)\left(\exists^{\mu} i<\mu\right)\left(c_{\delta^{q}}\left(\nu_{\varepsilon(i)}^{\delta^{q}}\right)=\xi\right)$ and for $\mu$ ordinals $i<\mu$ divisible by $\zeta$,

$$
(\forall j<\zeta)\left(c_{\delta^{q}}\left(\nu_{\varepsilon(i+j)}^{\delta^{q}}=\alpha_{j}^{0}\right) .\right.
$$

The construction is easy and can be done as in 2.3.6. Next we put

$$
\begin{gathered}
t^{q}=t^{p} \cup\left\{\varrho \mid \gamma: \gamma \leq \delta^{q}\right\} \cup\left\{\nu_{\beta}^{\gamma}: \beta<\mu, \delta^{p}<\gamma \leq \delta^{q}\right\}, \\
w^{q}=w^{p}, \quad D^{q}=D^{p}, \quad \bar{d}^{q}=\bar{d}^{p} .
\end{gathered}
$$

The function $c^{q}$ is defined as any extension of $c^{p} \cup\left\{c_{\gamma}: \delta^{p}<\gamma \leq \delta^{q}\right\}$ (note that the possibly undefined values are in some initial segments of $\varrho$ ). For $\alpha \in w^{q}$ let $b_{\alpha}^{q}$ be $\nu_{\beta}^{\delta^{q}}$ for $\beta$ such that $b_{\alpha}^{p}=\nu_{\beta}$. This determines the tree ordering $\leq^{q}$ of $t^{q} \cup w^{q}$ (remember 2.5.3(C)). To define $e_{\bar{x}, h}^{q}$ we let:

- $e_{\bar{x}, h}^{q} \supseteq e_{\bar{x}, h}^{p}$,

- $\operatorname{dom}\left(e_{\bar{x}, h}^{q}\right)=\operatorname{dom}\left(e_{\bar{x}, h}^{p}\right) \cup\left\{(\bar{y}, \iota): \bar{y}=\left\langle\nu_{\beta(i)}^{\gamma}: i<\mu\right\rangle>\bar{y}^{\prime}=\left\langle\nu_{\beta(i)}: i<\mu\right\rangle\right.$, $\left.\gamma \in\left(\delta^{p}, \delta^{q}\right], \quad\left(\bar{y}^{\prime}, \iota\right) \in \operatorname{dom}\left(e_{\bar{x}, h}^{p}\right)\right\}$

- if $\bar{x}<\bar{y}^{\prime}<\bar{y},\left(\bar{y}^{\prime}, \iota\right) \in \operatorname{dom}\left(e_{\bar{x}, h}^{p}\right)$ and $\operatorname{lev}\left(\bar{y}^{\prime}\right)=\delta^{p}$ then $e_{\bar{x}, h}^{q}(\bar{y}, \iota)=$ $e_{\bar{x}, h}^{p}\left(\bar{y}^{\prime}, \iota\right)$.

Now one easily checks that the condition $q$ defined above is as required.

(2) Choose $\nu \in \delta^{\delta^{p}} \lambda$ such that $\nu\left\lceil 1 \notin t^{p}\right.$. Let

$$
\delta^{q}=\delta^{p}, \quad t^{q}=t^{p} \cup\left\{\nu\left\lceil\gamma: \gamma<\delta^{p}\right\}, \quad w^{q}=w^{p} \cup\{\beta\} .\right.
$$


Define $\leq^{q}$ by letting $b_{\alpha}^{q}=b_{\alpha}^{p}$ for $\alpha \in w^{p}$ and $b_{\beta}^{q}=\left\{\nu\left\lceil\varepsilon: \varepsilon<\delta^{q}\right\}\right.$. Finally, put $D^{q}=D^{p}, d_{\bar{x}, h}^{q}=d_{\bar{x}, h}^{p}$ and $e_{\bar{x}, h}^{q}=e_{\bar{x}, h}^{q}$.

(3) Let $t^{q}=t^{p}, w^{q}=w^{p}, c^{q}=c^{p}, d_{\bar{x}^{\prime}, h^{\prime}}^{q}=d_{\bar{x}^{\prime}, h^{\prime}}^{p}$ if $\left(\bar{x}^{\prime}, h^{\prime}\right) \in D^{p}, d_{\bar{x}, h}^{q}$ is empty if $(\bar{x}, h) \notin D^{p}$, and similarly for $e_{\bar{x}^{\prime}, h^{\prime}}^{q}$

(4) Let $h=\left\langle\left(\alpha_{\varepsilon}^{0}, \alpha_{\varepsilon}^{1}, \alpha_{\varepsilon}^{2}\right): \varepsilon<\zeta\right\rangle$. Declare that $\delta^{q}=\delta^{p}+\omega$ and fix an enumeration $\left\langle\nu_{\beta}: \beta<\mu\right\rangle$ of $t^{p} \cap{ }^{\delta^{p}} \lambda$. Let $\bar{y}^{\prime}=\left\langle\nu_{\beta(i)}: i<\mu\right\rangle$ be such that $\bar{y}^{\prime}<\bar{y}\left(\in w^{[\mu]}\right)$. Next, as in (1) above build $c_{\gamma}$ and $\nu_{\beta}^{\gamma}$ for $\gamma \in\left(\delta^{p}, \delta^{q}\right]$ and $\beta<\mu$ satisfying demands (a) $-(\mathrm{c})$ there plus:

(c) $(\delta)$ for each $\gamma \in\left(\delta^{p}, \delta^{q}\right)$,

$$
(\forall \xi<\mu)\left(\exists^{\mu} i<\mu\right)\left(c_{\gamma}\left(\nu_{\beta(i)}^{\gamma}\right)=\xi\right)
$$

and for $\mu$ ordinals $i<\mu$ divisible by $\zeta$ there is $\xi<\lambda$ such that

$$
(\forall j<\zeta)\left(c_{\gamma}\left(\nu_{\beta(i+j)}^{\gamma}\right)=\alpha_{j}^{0} \& \nu_{\beta(i+j)}^{\gamma+1}(\gamma)=\zeta \cdot \xi+\alpha_{j}^{1}\right),
$$

( $\varepsilon)(\forall \xi<\mu)\left(\exists^{\mu} i<\mu\right)\left(c_{\delta^{q}}\left(\nu_{\beta(i)}^{\delta^{q}}\right)=\xi\right)$ and for $\mu$ ordinals $i<\mu$ divisible by $\zeta$,

$$
(\forall j<\zeta)\left(c_{\delta^{q}}\left(\nu_{\beta(i+j)}^{\delta^{q}}=\alpha_{j}^{0}\right) .\right.
$$

Next we put $\operatorname{dom}\left(d_{\bar{x}, h}^{q}\right)=\operatorname{dom}\left(d_{\bar{x}, h}^{p}\right) \cup\{\bar{y}\}, d_{\bar{x}, h}^{q} \supseteq d_{\bar{x}, h}^{p}, d_{\bar{x}, h}^{q}(\bar{y})=\delta^{p}+2$, $d_{\bar{x}^{\prime}, h^{\prime}}^{q}=d_{\bar{x}^{\prime}, h^{\prime}}^{p}$ for $\left(\bar{x}^{\prime}, h^{\prime}\right) \in D^{p} \backslash\{(\bar{x}, h)\}$, and $D^{q}=D^{p}$. The functions $c^{q}$ and $e_{\bar{x}^{\prime}, h^{\prime}}^{q}\left(\right.$ for $\left.\left(\bar{x}^{\prime}, h^{\prime}\right) \in D^{q}\right)$ are defined as in (1), but dealing with $(\bar{x}, h)$ we take into account the new obligation: $d_{\bar{x}, h}^{q}(\bar{y})=\delta^{p}+2$ (and we put the value 2 whenever possible). There is no problem with it as we demanded clauses $(\mathrm{c})(\delta, \varepsilon)$. Now one easily checks that $q$ is as required.

Claim 2.5.6. The forcing notion $\mathbb{P}$ is $(<\lambda)$-complete, i.e. if $\bar{p}=\left\langle p^{i}\right.$ : $\left.i<i^{*}\right\rangle \subseteq \mathbb{P}$ is increasing and $i^{*}<\lambda$ then $\bar{p}$ has an upper bound in $\mathbb{P}$.

Pr o of. It is easy: the first candidate for the upper bound is the natural union of the $p^{i}$ 's. What may fail is that the tree $\bigcup_{i<i^{*}} t^{p^{i}}$ does not have the last level. But this is not a problem as we may use the procedure of 2.5.5(1) to add it.

Claim 2.5.7 (The Amalgamation Property). If $\alpha<\lambda^{+}$is either a successor ordinal or of cofinality $\lambda, p, q \in \mathbb{P}, p\left\lceil\alpha \leq q\right.$ and $w^{q} \subseteq \alpha$ then there is $r \in \mathbb{P}$ such that $p \leq r, q \leq r$ and $w^{r}=w^{p} \cup w^{q}$.

Proof. By 2.5.5(1) we may assume that $\delta^{p}<\delta^{q}$. Moreover, we may assume that $\left|w^{p} \backslash \alpha\right|=\mu$ (as otherwise everything is easier). Let $\delta^{r}=\delta^{q}$ and $w^{r}=w^{p} \cup w^{q}$. By induction on $\gamma \in\left[\delta^{p}, \delta^{q}\right]$ choose sequences $\left\langle\nu_{\beta, \gamma}: \beta \in\right.$ $\left.w^{p} \backslash \alpha\right\rangle$ and functions $c_{\gamma}$ such that: 
( $\alpha) \nu_{\beta, \gamma} \in{ }^{\gamma} \lambda$ are $\triangleleft$-increasing with $\gamma$,

( $\beta) \nu_{\beta, \delta^{p}}=b_{\beta}^{p}$ and $\nu_{\beta, \delta^{p}+1} \notin t^{q}$ [note that $\left\langle\nu_{\beta, \delta^{p}}: \beta \in w^{p} \backslash \alpha\right\rangle$ is with no repetition],

$(\gamma) c_{\gamma}:\left\{\nu_{\beta, \xi}: \beta \in w^{p} \backslash \alpha, \xi \in\left[\delta^{p}, \gamma\right)\right\} \rightarrow \lambda$ are continuously increasing with $\gamma$ and $c_{\delta^{p}+1}$ is $c^{p}$ restricted to $\left\{\nu_{\beta, \delta^{p}}: \beta \in w^{p} \backslash \alpha\right\}$,

and for each $(\bar{x}, h) \in D^{p}, h=\left\langle\left(\alpha_{\varepsilon}^{0}, \alpha_{\varepsilon}^{1}, \alpha_{\varepsilon}^{2}\right): \varepsilon<\zeta\right\rangle, \bar{z} \in \operatorname{dom}\left(d_{\bar{x}, h}^{p}\right) \backslash$ $\operatorname{dom}\left(d_{\bar{x}, h}^{q}\right)$ and $i^{*}<\mu$ such that $z_{i} \geq \alpha$ for $i \geq i^{*}$ we have:

$(\delta)$ for each $\gamma \in\left[\delta^{p}, \delta^{q}\right)$, for $\mu$ ordinals $i \in\left[i^{*}, \mu\right)$ divisible by $\zeta$, for some $\xi<\lambda$,

$$
(\forall \varepsilon<\zeta)\left(c_{\gamma+1}\left(\nu_{z_{i+\varepsilon}, \gamma}\right)=\alpha_{\varepsilon}^{0} \& \nu_{z_{i+\varepsilon}, \gamma+1}(\gamma)=\zeta \cdot \xi+\alpha_{\varepsilon}^{1}\right)
$$

$(\varepsilon)$ for $\mu$ ordinals $i \in\left[i^{*}, \mu\right)$ divisible by $\zeta$,

$$
(\forall \varepsilon<\zeta)\left(c_{\delta^{q}+1}\left(\nu_{z_{i+\varepsilon}, \delta^{q}}\right)=\alpha_{\varepsilon}^{0}\right),
$$

$(\zeta)$ if $\bar{x}<\bar{y}<\bar{z}, \operatorname{lev}(\bar{y})=\delta^{p}, \bar{y}<\bar{y}^{\prime}, \operatorname{lev}\left(\bar{y}^{\prime}\right)=\delta^{p}+1,\left(\bar{y}^{\prime}, d_{\bar{x}, h}^{p}(\bar{z})\right) \in$ $\operatorname{dom}\left(e_{\bar{x}, h}^{q}\right)$ and $e_{\bar{x}, h}^{q}\left(\bar{y}^{\prime}, d_{\bar{x}, h}^{p}(\bar{z})\right)=0$ then for $\mu$ ordinals $i \in\left[i^{*}, \mu\right)$ divisible by $\zeta$ there are $\xi_{0}<\xi_{1}<\lambda$ such that

$$
\begin{gathered}
(\forall \varepsilon<\zeta)\left(\zeta \cdot \xi_{0} \leq y_{i+\varepsilon}^{\prime}\left(\delta^{p}\right)<\zeta \cdot \xi_{1} \leq y_{i+\varepsilon}\left(\delta^{p}\right)\right), \\
(\forall \varepsilon<\zeta)\left(c^{p}\left(\nu_{z_{i+\varepsilon}, \delta^{p}}\right)=\alpha_{\varepsilon}^{0}, y_{i+\varepsilon}^{\prime}\left(\delta^{p}\right)=\zeta \cdot \xi_{0}+\alpha_{\varepsilon}^{1}, y_{i+\varepsilon}\left(\delta^{p}\right)=\zeta \cdot \xi_{1}+\alpha_{\varepsilon}^{2}\right),
\end{gathered}
$$

(८) for each $\gamma \in\left(\delta^{p}, \delta^{q}\right]$, for every $\varepsilon<\mu$ there are $\mu$ ordinals $i<\mu$ such that $c_{\gamma+1}\left(\nu_{z_{i}, \gamma}\right)=\varepsilon$.

[Our intention here is that $b_{\beta}^{r}=\nu_{\beta, \delta^{q}}$ and $c^{r} \supseteq c_{\delta^{q}}$.] We have actually $\mu$ demands, each of which can be satisfied by $\mu$ pairwise disjoint cases of size $\zeta<\mu$. So we may carry out the procedure analogous to that from the end of the proof of 2.3.7. Note that in handling instances of clause $(\zeta)$ we use demand 2.5.3(G)(v) for $q$ (applicable as $d_{\bar{x}, h}^{p}(\bar{z})<\delta^{p}$ ) and for clause $(\delta)$ we use 2.5.3(G)(iv). After the construction is carried out we easily define a condition $r$ as required. $\mathbf{m}_{2.5 .7}$

Claim 2.5.8. The forcing notion $\mathbb{P}$ satisfies the $\lambda^{+}$-cc.

Proof. Suppose that $\left\langle p_{\alpha}: \alpha<\lambda^{+}\right\rangle$is an antichain in $\mathbb{P}$. By passing to a subsequence we may assume that $\operatorname{otp}\left(w^{p_{\alpha}}\right)$ is constant and that the order isomorphism of $w^{p_{\alpha}}, w^{p_{\beta}}$ carries the condition $p_{\alpha}$ to $p_{\beta}$ ( so $t^{p_{\alpha}}=t^{p_{\beta}}$, $D^{p_{\alpha}}=D^{p_{\beta}}$ etc.). Moreover, we may assume that the family $\left\{w^{p_{\alpha}}: \alpha<\lambda^{+}\right\}$ forms a $\Delta$-system with kernel $w^{*}$ (remember $\lambda=2^{\mu}=\mu^{+}$). Now we may find an ordinal $\alpha^{*}<\lambda^{+}$of cofinality $\lambda$ and $\alpha_{0}<\alpha_{1}<\lambda^{+}$such that $w^{p_{\alpha_{0}}} \subseteq \alpha^{*}, w^{p_{\alpha_{1}}} \cap \alpha^{*}=w^{*}$ and $w^{*}$ is an initial segment of both $w^{p_{\alpha_{0}}}$ and $w_{p_{\alpha_{1}}}$. Note that then $p_{\alpha_{1}}\left\lceil\alpha^{*} \geq p_{\alpha_{0}}\right.$. So applying 2.5.7 we conclude that the conditions $p_{\alpha_{0}}, p_{\alpha_{1}}$ have a common upper bound, a contradiction. $\mathbf{-}_{2.5 .8}$ 
To finish the proof note that if $G \subseteq \mathbb{P}$ is a generic filter over $\mathbf{V}$, then in $\mathbf{V}[G]$ we may define the tree $T$ by

$$
T=\left({ }^{\lambda>} \lambda\right) \cup\left\{\eta_{\alpha}: \alpha<\lambda^{+}\right\}
$$

where for $\alpha<\lambda^{+}$we define $\eta_{\alpha} \in{ }^{\lambda} \lambda$ by

$$
\eta_{\alpha}=\bigcup\left\{\nu \in{ }^{\lambda>\lambda} \lambda:(\exists p \in G)\left(\nu \in b_{\alpha}^{p}\right)\right\}
$$

(and $c, d$ are defined similarly). By 2.5.5 and 2.5.6 (no new $\mu$-sequences of ordinals are added) we easily conclude that these objects are as needed.

$\mathbf{\square}_{2.5}$

We may want to improve 2.3(2) so that it looks more like 2.5(4); we can do it at some price.

Proposition 2.6. Let $\mathcal{J}^{*}$ be a linear order, $\mathcal{J}^{*}=\sum_{\alpha<\lambda^{+}} \mathcal{I}_{\alpha}$, with each $\mathcal{I}_{\alpha}$ a $\lambda$-dense linear order of cardinality $\lambda$ (as in the proof of 2.3(1)). Then $\mathcal{J}=\omega \times \mathcal{J}^{*}$ is a $\lambda^{+}$-like linear order such that every $j \in \mathcal{J}$ which is neither a successor nor the first element $\left(\right.$ under $\left.<_{\mathcal{J}}\right)$ satisfies

$$
\operatorname{cf}\left(\left\{i: i<_{\mathcal{J}} j\right\},<_{\mathcal{J}}\right)=\lambda
$$

and each member of $\mathcal{J}$ has an immediate successor.

Definition 2.7. For a $\lambda^{+}$-like linear order $\mathcal{J}$, a $\mathcal{J}$-Aronszajn tree is $T=\left(T, \leq_{T}\right.$, lev $)$ such that:

(a) $T$ is a set of cardinality $\lambda^{+}$,

(b) $\left(T, \leq_{T}\right)$ is a partial order which is a tree, i.e. for every $y \in T$ the set $\left\{x: x \leq_{T} y\right\}$ is linearly ordered by $\leq_{T}$,

(c) lev is a function from $T$ to $\mathcal{J}$, and $T_{j}:=\{y \in T: \operatorname{lev}(y)=j\}$,

(d) for every $y \in T$, lev is a one-to-one order preserving function from $\left\{x: x<_{T} y\right\}$ onto $\left\{j \in \mathcal{J}: j<_{\mathcal{J}} \operatorname{lev}(y)\right\}$, so $y\lceil j$ is naturally defined,

(e) for $y \in T$ and $j \in \mathcal{J}$ with $\operatorname{lev}(y)<_{\mathcal{J}} j$ there is $z$ such that $y<_{T} z \in T$ and $\operatorname{lev}(z)=j$,

(f) $\{y: \operatorname{lev}(y)=j\}$ has cardinality $\lambda$,

(g) normality: if $y \neq z$, both in $T_{j}$, and $j$ is neither a successor nor the first element of $\left(\mathcal{J},<_{\mathcal{J}}\right)$ then $\left\{x: x<_{T} y\right\} \neq\left\{x: x<_{T} z\right\}$,

(h) for $y \neq z \in T$ there is $j \in \mathcal{J}$ such that $y\lceil j=z\lceil j$ and

$$
(\forall i)\left(j<_{\mathcal{J}} i \Rightarrow y\lceil i \neq z\lceil i)\right.
$$

[we write $z \wedge y$ for $y\lceil j=z\lceil j]$.

Theorem 2.8. Assume that $\lambda=\mu^{+}=2^{\mu}$ and $\nabla_{\lambda}$ (the second follows e.g. if $\mu \geq \beth_{\omega}$; see $\left.[\mathrm{Sh} 460,3.5(1)]\right)$ and $\mathcal{J}$ is as constructed in 2.6. Then there are a $\mathcal{J}$-Aronszajn tree $T$ and functions $f, c, d$ such that:

(a) $f, c$ are functions from $T$ to $\lambda$, and if $i$ is the successor of $j$ in $\mathcal{J}$ and $y \in T_{i}$ then $f$ is one-to-one from $\left\{z \in T_{j}: z\lceil i=y\}\right.$ onto $\lambda$, 
(b) for every $\bar{x} \in T^{[\mu]}\left(=\bigcup_{j \in \mathcal{J}} T_{j}^{[\mu]}\right)$ and $h: \zeta \rightarrow \lambda \times \lambda \times \lambda$ with $\zeta<\mu$ we have $d_{\bar{x}, h}: T_{\bar{x}}^{[\mu]} \rightarrow \lambda$ such that if $d_{\bar{x}, h}(\bar{x})=d_{\bar{x}, h}(\bar{y})$ then for some $\bar{t} \in T_{\bar{x}}^{[\mu]}$ we have:

( $\alpha) t_{i}=y_{i} \wedge z_{i}$

( $\beta) \operatorname{lev}(\bar{t})<\operatorname{lev}(\bar{y})$ and $\operatorname{lev}(\bar{t})<\operatorname{lev}(\bar{z})$,

$(\gamma)(\forall \varepsilon<\mu)\left(\exists^{\mu} i<\mu\right)\left(c\left(t_{i}\right)=\varepsilon\right)$,

$(\delta)$ for $\mu$ ordinals $i<\mu$ divisible by $\zeta$ we have

$$
h(\zeta)=\left\langle\left(c\left(t_{i}\right), f\left(y_{i} \uparrow(\alpha+\mathcal{J} 1)\right), f\left(z_{i} \uparrow(\alpha+\mathcal{J} 1)\right)\right),\right.
$$

where $\alpha=\operatorname{lev}(\bar{t})$.

Proof. Like 2.3.

\section{Constructions related to pcf theory}

Lemma 3.1. (1) Suppose that:

(A) $\left\langle\lambda_{i}: i<\delta\right\rangle$ is a strictly increasing sequence of regular cardinals, $|\delta|<\lambda_{i}<\lambda=\operatorname{cf}(\lambda)$ for $i<\delta$ and $D$ is a $\sigma$-complete filter on $\delta$ containing all co-bounded subsets of $\delta$ (follows by clause (D); hence $\operatorname{cf}(\delta) \geq \sigma)$,

(B) $\operatorname{tcf}\left(\prod_{i<\delta} \lambda_{i} / D\right)=\lambda$, i.e. there is a sequence $\left\langle f_{\alpha}: \alpha<\lambda\right\rangle \subseteq$ $\prod_{i<\delta} \lambda_{i}$ such that:

(i) $\alpha<\beta<\lambda$ implies $f_{\alpha}<_{D} f_{\beta}$,

(ii) $\left(\forall f \in \prod_{i<\delta} \lambda_{i}\right)(\exists \alpha<\lambda)\left(f<{ }_{D} f_{\alpha}\right)$,

(C) sets $A_{i} \subseteq \delta($ for $i<\kappa)$ are such that the family $\left\{A_{i}: i<\kappa\right\}$ is $\sigma$-independent in $\mathcal{P}(\delta) / D$ (i.e. if $u, v$ are disjoint subsets of $\kappa$ with $|u \cup v|<\sigma$ then $\left.\bigcap_{i \in u} A_{i} \backslash \bigcup_{j \in v} A_{j} \neq \emptyset \bmod D\right)$,

(D) $\left|\left\{f_{\alpha} \mid i: \alpha<\lambda\right\}\right|^{<\sigma}<\lambda_{i}$ for each $i<\delta$.

Then $\operatorname{Ens}_{\sigma}(\lambda, \kappa)$.

(2) The linear orders in part (1) have exact density $\mu:=\sum_{i<\delta} \lambda_{i}$ (see Definition 1.9) and they are positively $\mu$-entangled (see Definition 1.10). Moreover, if $\left\langle f_{\alpha}: \alpha<\lambda\right\rangle$ is as in [Sh 355, §1] (i.e. it is $\mu$-free) then they have exact density $\left(\mu^{+}, \mu^{+}, \mu\right)$.

Remark. By [Sh 355, 3.5], if $\delta<\lambda_{0}$ and $\max \operatorname{pcf}\left(\left\{\lambda_{i}: i<\alpha\right\}\right)<\lambda_{\alpha}=$ $\operatorname{cf}\left(\lambda_{\alpha}\right)($ for $\alpha<\delta)$ and $(*) \lambda=\operatorname{cf}\left(\prod_{\alpha<\delta} \lambda_{\alpha},<_{D}\right)$ then we can have (B) $+(\mathrm{D})$; i.e. we can find $f_{\alpha}$ (for $\alpha<\lambda$ ) satisfying (i)+(ii) of (B) and (D). If instead of $(*)$ we have $\lambda \in \operatorname{pcf}_{\sigma \text {-complete }}\left\{\lambda_{i}: i<\delta\right\}$ (but $\lambda \notin \operatorname{pcf}\left(\left\{\lambda_{i}: i<\alpha\right\}\right)$ for $\alpha<\lambda)$ then we can find a filter $D$ as required in $(\mathrm{A})+(\mathrm{B})+(\mathrm{C})$. So if $\mu>\operatorname{cf}(\mu)=\kappa$ and $\alpha<\mu \Rightarrow|\alpha|^{\kappa}<\mu$, then we can find $\bar{\lambda}=\left\langle\lambda_{i}: i<\kappa\right\rangle$ as above and $\bar{\lambda}$ strictly increasing with limit $\mu$ with $D$ the family of co-bounded 
subsets of $\kappa$, for some $\lambda \in\left(\mu, \mu^{\kappa}\right]$. If $\kappa>\aleph_{0}$, for any regular such $\lambda$ there is such $\bar{\lambda}$ (see [Sh:g, VIII, §1]).

Proof of Lemma 3.1. (1) Let $\mathcal{I}=\left\{f_{\alpha}: \alpha<\lambda\right\}$. For each $\zeta<\kappa$ we define a linear order $<_{\zeta}^{*}$ of $\mathcal{I}$. It is $<_{A_{\zeta}}^{*}$, where for $A \subseteq \delta$ we define $<_{A}^{*}$ by $f_{\alpha}<_{A}^{*} f_{\beta}$ if and only if

$$
(\exists i<\delta)\left(f_{\alpha}(i) \neq f_{\beta}(i) \& f_{\alpha}\left\lceil i=f_{\beta}\left\lceil i \&\left[f_{\alpha}(i)<f_{\beta}(i) \Leftrightarrow i \in A\right]\right) .\right.\right.
$$

Let $u, v$ be disjoint subsets of $\kappa$ with $|u \cup v|<\sigma$ and for each $\varepsilon \in u \cup v$ let $t_{\alpha}^{\varepsilon}=f_{\gamma(\varepsilon, \alpha)}$ be pairwise distinct (for $\alpha<\lambda$ ). We should find $\alpha<\beta<\lambda$ as in 1.1(1). Let

$$
\begin{aligned}
g_{\alpha}(i) & :=\min \left\{f_{\gamma(\varepsilon, \alpha)}(i): \varepsilon \in u \cup v\right\}, \\
i_{\alpha} & :=\min \left\{i<\delta:\left\langle f_{\gamma(\varepsilon, \alpha)}\lceil i: \varepsilon \in u \cup v\rangle \text { are pairwise distinct }\right\} .\right.
\end{aligned}
$$

Since $|u \cup v|<\sigma \leq \operatorname{cf}(\delta)$, we have $i_{\alpha}<\delta$. Clearly $g_{\alpha} \in \prod_{i<\delta} \lambda_{i}$. Without loss of generality $i_{\alpha}=i^{*}$ for every $\alpha<\lambda$. Let

$$
B=\left\{i<\delta:\left(\forall \xi<\lambda_{i}\right)\left(\exists^{\lambda} \alpha<\lambda\right)\left(g_{\alpha}(i)>\xi\right)\right\} .
$$

Claim 3.1.1. $B \in D$.

Proof. Assume not, so $\delta \backslash B \neq \emptyset \bmod D$. For $i \in \delta \backslash B$ let $\xi_{i}<\lambda_{i}$ and $\beta_{i}<\lambda$ exemplify $i \notin B$, i.e. $\alpha \in\left[\beta_{i}, \lambda\right) \Rightarrow g_{\alpha}(i) \leq \xi_{i}$. Define $h \in \prod_{i<\delta} \lambda_{i}$ by

$$
h(i):= \begin{cases}\xi_{i}+1 & \text { if } i \in \delta \backslash B, \\ 0 & \text { if } i \in B .\end{cases}
$$

Now $\left\langle f_{\alpha} / D: \alpha<\lambda\right\rangle$ is cofinal in $\prod_{i<\delta} \lambda_{i} / D$ (clause (ii) of (B)), so there exists $\beta<\lambda$ such that $h<f_{\beta} \bmod D$. Without loss of generality $\sup _{i \in \delta \backslash B} \beta_{i}<$ $\beta$ (remember that $\delta \backslash B \subseteq \delta,|\delta|<\lambda=\operatorname{cf}(\lambda)$ and $(\forall i \in \delta \backslash B)\left(\beta_{i}<\lambda\right)$ ). Since, for each $\varepsilon \in u \cup v, \gamma(\varepsilon, \alpha)$ (for $\alpha<\lambda$ ) are pairwise distinct and $\beta<\lambda$, there exists $\alpha<\lambda$ such that $(\forall \varepsilon \in u \cup v)(\gamma(\varepsilon, \alpha)>\beta)$. Without loss of generality $\beta<\alpha$ and hence $\sup _{i \in \delta \backslash B} \beta_{i}<\alpha$. Now by the choice of $\alpha$ we have $(\forall \varepsilon \in u \cup v)\left(f_{\beta}<f_{\gamma(\varepsilon, \alpha)} \bmod D\right)$ and for every $i \in \delta \backslash B, g_{\alpha}(i) \leq \xi_{i}$. Hence $E_{\varepsilon}:=\left\{i<\delta: f_{\beta}(i)<f_{\gamma(\varepsilon, \alpha)}(i)\right\} \in D$ and as $D$ is $\sigma$-complete and $\sigma>|u \cup v|$ we get $\bigcap_{\varepsilon \in u \cup v} E_{\varepsilon} \in D$. By $g_{\alpha}$ 's definition and the choice of $\beta$, it now follows that $\left\{i<\delta: h(i)<g_{\alpha}(i)\right\} \in D$ and thus

$$
(\delta \backslash B) \cap\left\{i<\delta: h(i)<g_{\alpha}(i)\right\} \neq \emptyset \bmod D .
$$

Choosing $i$ in this (non-empty) intersection, one obtains $g_{\alpha}(i) \leq \xi_{i}<\xi_{i}+1=$ $h(i)<g_{\alpha}(i)$ (the first inequality - see above, the third equality - see choice of $h$, the last inequality - see choice of $i$ ), a contradiction. So $B \in D$, proving the claim. $\mathbf{-}_{3.1 .1}$

Remember that $\left|\left\{f_{\alpha} \mid i: \alpha<\lambda\right\}\right|<\lambda_{i}$ for each $i<\delta$, and $\operatorname{cf}\left(\prod_{i<\delta} \lambda_{i} / D\right)$ $=\lambda, D$ contains all co-bounded subsets of $\delta$. By our hypothesis, 


$$
A:=\bigcap_{\varepsilon \in u} A_{\varepsilon} \cap \bigcap_{\varepsilon \in v}\left(\delta \backslash A_{\varepsilon}\right) \neq \emptyset \bmod D,
$$

so $C:=\left\{i<\delta: i^{*}<i\right\} \cap A \cap B \neq \emptyset \bmod D$, and one can choose $i \in C$. For each $\xi<\lambda_{i}$ choose $\alpha_{\xi}<\lambda$ such that $g_{\alpha_{\xi}}(i)>\xi$. Then clearly for some unbounded $S \subseteq \lambda_{i}$ we have

$$
\xi_{1}<\xi_{2} \in S \& \varepsilon_{1}, \varepsilon_{2} \in u \cup v \Rightarrow f_{\gamma\left(\varepsilon_{1}, \alpha_{\xi_{1}}\right)}(i)<f_{\gamma\left(\varepsilon_{2}, \alpha_{\xi_{2}}\right)}(i) .
$$

Without loss of generality the sequence $\left\langle\left\langle f_{\gamma\left(\varepsilon, \alpha_{\xi}\right)}\lceil i: \varepsilon \in u \cup v\rangle: \xi \in S\right\rangle\right.$ is constant (by hypothesis (D) of 3.1(1)). The conclusion should be clear now (look at the definition of $<_{\zeta}^{*}$ and the choice of $i$ in $\bigcap_{\varepsilon \in u} A_{\varepsilon} \backslash \bigcup_{\varepsilon \in v} A_{\varepsilon}$ ).

(2) We will state the requirements and prove them one by one.

Claim 3.1.2. The linear orders constructed in the first part have exact density $\mu$.

P r o of. Consider $\mathcal{I}=\left(\mathcal{I},<_{A}\right)$. For each $i<\delta$ choose a set $X_{i} \subseteq \lambda$ such that $\left|X_{i}\right| \leq \lambda_{i}$ and $\left\{f_{\alpha}\lceil i: \alpha<\lambda\}=\left\{f_{\alpha}\left\lceil i: \alpha \in X_{i}\right\}\right.\right.$. Then $\left\{f_{\alpha}: \alpha \in\right.$ $\left.\bigcup_{i<\delta} X_{i}\right\}$ is a dense subset of $\left(\mathcal{I},<_{A}\right)$ (and its size is $\leq \mu$ ).

Suppose now that $\mathcal{J} \subseteq \mathcal{I},|\mathcal{J}|=\lambda$ and assume that $\mathcal{J}_{0} \subseteq \mathcal{J}$ is a dense subset of $\mathcal{J}$ with $\left|\mathcal{J}_{0}\right|<\mu$. The set $X=\left\{\alpha<\lambda: f_{\alpha} \in \mathcal{J}\right\}$ has cardinality $\lambda$, so it is unbounded in $\lambda$. Let $i(*)=\min \left\{i<\delta: \lambda_{i}>\left|\mathcal{J}_{0}\right|\right\}$. Then

$$
(\forall i \geq i(*))\left(\gamma_{i}:=\sup \left\{f_{\alpha}(i)+1: f_{\alpha} \in \mathcal{J}_{0}\right\}<\lambda_{i}\right)
$$

(as $\gamma_{i}$ is a supremum of a set of $\left|\mathcal{J}_{0}\right|<\lambda_{i}=\operatorname{cf}\left(\lambda_{i}\right)$ ordinals $<\lambda_{i}$ ). Let $\gamma_{i}=0$ for $i<i(*)$. Then $\left\langle\gamma_{i}: i<\delta\right\rangle \in \prod_{i<\delta} \lambda_{i}$ and, as $\left\langle f_{\alpha}: \alpha<\lambda\right\rangle$ is cofinal in $\left(\prod_{i<\delta} \lambda_{i},<_{D}\right)$, for some $\alpha(*)<\lambda$ we have $\left\langle\gamma_{i}: i<\delta\right\rangle<_{D} f_{\alpha(*)}$. Since $(\forall \alpha \in X \backslash \alpha(*))\left(f_{\alpha(*)} \leq_{D} f_{\alpha}\right)$, we have

$$
(\forall \alpha \in X \backslash \alpha(*))\left(\left\{i<\delta: \gamma_{i}<f_{\alpha}(i)\right\} \in D\right) .
$$

Consequently, for each $\alpha \in X \backslash \alpha(*)$ we find $i_{\alpha} \in(i(*), \delta)$ such that $f_{\alpha}\left(i_{\alpha}\right)$ $>\gamma_{i_{\alpha}}$. As $\lambda=\operatorname{cf}(\lambda)>|\delta|$, there is $j \in(i(*), \delta)$ such that the set

$$
X^{\prime}:=\left\{\alpha \in X: \alpha>\alpha(*) \& i_{\alpha}=j\right\}
$$

is unbounded in $\lambda$. Since $\left|\left\{f_{\alpha} \mid j: \alpha<\lambda\right\}\right|<\lambda_{j}<\lambda=\operatorname{cf}(\lambda)$, for some unbounded set $X^{\prime \prime} \subseteq X^{\prime}$ and a sequence $\nu$ we have $\left(\forall \alpha \in X^{\prime \prime}\right)\left(f_{\alpha}\lceil j=\nu)\right.$. But now note that the convex hull of $\left\{f_{\alpha}: \alpha \in X^{\prime \prime}\right\}$ in $\left(\mathcal{I},<_{A}\right)$ is disjoint from $\mathcal{J}_{0}$, a contradiction. $\mathbf{-}_{3.1 .2}$

Claim 3.1.3. $\left(\mathcal{I},<_{A}\right)$ is positively $\sigma$-entangled.

Proof. As in part (1).

Claim 3.1.4. If the sequence $\left\langle f_{\alpha}: \alpha<\lambda\right\rangle$ is $\mu$-free and the set $A \subseteq \delta$ is neither bounded nor co-bounded then the linear order $\left(\mathcal{I},<_{A}\right)$ has exact density $\left(\mu^{+}, \mu^{+}, \mu\right)$. 
Pr o of. Suppose that $\mathcal{J} \subseteq \mathcal{I}$ is of size $\geq \mu^{+}$. By 3.1.2 its density is $\leq \mu$. For the other inequality suppose that $\mathcal{J}_{0}$ is a dense subset of $\mathcal{J}$ of cardinality $<\mu$. Let

$\mathcal{J}^{\prime}=\left\{f_{\alpha} \in \mathcal{J}\right.$ : for each $i<\delta$ there are $\beta_{1}, \beta_{2}$ such that $f_{\beta_{1}}, f_{\beta_{2}} \in \mathcal{J}$ and

$$
f_{\beta_{1}}\left\lceil i=f_{\alpha}\left\lceil i=f_{\beta_{2}}\left\lceil i \& f_{\beta_{1}}<_{A} f_{\alpha}<_{A} f_{\beta_{2}}\right)\right\} .\right.
$$

Plainly $\left|\mathcal{J} \backslash \mathcal{J}^{\prime}\right| \leq \mu$, so $\left|\mathcal{J}^{\prime}\right| \geq \mu^{+}$. Since $\theta:=\left|\mathcal{J}_{0}\right|<\mu$ and $\mu$ is a limit cardinal, we have $\sigma=(\theta+|\delta|)^{+}<\mu$. Let $X=\left\{\alpha: f_{\alpha} \in \mathcal{J}^{\prime}\right\}$ and choose $X_{1} \subseteq X$ of size $\sigma$. Now we may find $\left\langle B_{\alpha}: \alpha \in X_{1}\right\rangle \subseteq D$ such that for each $j<\delta$ the sequence $\left\langle f_{\alpha}(j): \alpha \in X \& j \in B_{\alpha}\right\rangle$ is strictly increasing (or just without repetitions). Then for some $i(*)<\delta$ the set $X_{2}=\left\{\alpha \in X_{1}: i(*) \in B_{\alpha}\right\}$ has cardinality $\sigma$. But then the set

$$
X:=\left\{\alpha \in X_{2}: \neg\left(\exists f_{\beta} \in \mathcal{J}_{0}\right)\left(f_{\beta}\left\lceil(i(*)+1)=f_{\alpha}\lceil(i(*)+1))\right\}\right.\right.
$$

is of size $\sigma$ (remember $\left|\mathcal{J}_{0}\right|<\sigma=\operatorname{cf}(\sigma)$ ), a contradiction with the choice of $\mathcal{J}^{\prime} \cdot \mathbf{m}_{3.1 .4}$

This finishes the proof of the lemma. 3.1

Lemma 3.2. Suppose that $\mathfrak{a}$ is a set of regular cardinals satisfying $|\mathfrak{a}|^{+}<\min (\mathfrak{a}), \quad \lambda=\max \operatorname{pcf}(\mathfrak{a}) \quad$ and $\quad\left[\theta \in \mathfrak{a} \Rightarrow \theta>(\max \operatorname{pcf}(\theta \cap \mathfrak{a}))^{<\sigma}\right]$.

(1) Assume that $\kappa=|\mathfrak{a}|, \kappa=\kappa^{<\sigma}$ and $J$ is a $\sigma$-complete ideal on $\mathfrak{a}$ extending $J_{<\lambda}[\mathfrak{a}]$ and $\mathfrak{a}_{\varepsilon} \subseteq \mathfrak{a}$ are pairwise disjoint not in $J$ for $\varepsilon<\kappa$. If $2^{\kappa} \geq \lambda$ or just $2^{\kappa} \geq \sup (\mathfrak{a})$ then there is a $\sigma$-entangled linear order of power $\lambda$.

(2) We can replace " $\kappa=|\mathfrak{a}| "$ by $" \operatorname{cf}(\sup \mathfrak{a}) \leq \kappa "$.

Clearly in (1) and (2), $\mathfrak{a}$ has no last element and $|\mathfrak{a}| \geq \operatorname{cf}(\sup \mathfrak{a}) \geq \sigma$.

(3) If in (1) we omit the $\mathfrak{a}_{\varepsilon}$, still there is a positively $\sigma$-entangled linear order of power $\lambda$.

(4) The linear order above has exact density $\mu:=\sup \mathfrak{a}$. If there is a $\mu$-free sequence $\left\langle f_{\alpha}: \alpha<\lambda\right\rangle$ which is $<_{J_{<\lambda}[\mathfrak{a}]}$-increasing and cofinal (see [Sh 355, §1]) then the linear order has exact density $\left(\mu^{+}, \mu\right)$ (see Definition 1.9).

Proof. (1) This follows from part (2) as $\operatorname{cf}(\sup \mathfrak{a}) \leq|\mathfrak{a}| \leq \kappa$.

(2) Let $\left\langle f_{\alpha}: \alpha<\lambda\right\rangle$ be $\leq_{J_{<\lambda}[\mathfrak{a}]}$-increasing cofinal in $\prod \mathfrak{a} / J_{<\lambda}[\mathfrak{a}]$ with

$$
\left|\left\{f_{\alpha} \mid \theta: \alpha<\lambda\right\}\right| \leq \max \operatorname{pcf}(\mathfrak{a} \cap \theta) \quad \text { for } \theta \in \mathfrak{a}
$$

(exists by $\left[\right.$ Sh 355, 3.5]). For each $\theta \in \mathfrak{a}$ we can find sets $F_{\theta, \zeta}($ for $\zeta<\kappa)$ such that $F_{\theta, \zeta} \subseteq\left\{f_{\alpha}\lceil\theta: \alpha<\lambda\}\right.$, and for any disjoint subsets $X, Y$ of $\left\{f_{\alpha}\lceil\theta: \alpha<\lambda\}\right.$ of cardinality $<\sigma$, for some $\zeta<\kappa, F_{\theta, \zeta} \cap(X \cup Y)=X$ (possible as $\kappa=\kappa^{<\sigma}$ and $2^{\kappa} \geq\left|\left\{f_{\alpha} \mid \theta: \alpha<\lambda\right\}\right|$-by [EK] or see [Sh:g, AP1.10]). Clearly $\mathfrak{a}$ has no last element (as $J_{\mathfrak{a}}^{\text {bd }} \subseteq J$ and by the existence 
of the $\mathfrak{a}_{\varepsilon}$ 's) and $\operatorname{cf}(\sup \mathfrak{a}) \leq \kappa$, so there is an unbounded $\mathfrak{b} \subseteq \mathfrak{a}$ of cardinality $\leq \kappa$. As $\mathfrak{a}$ can be partitioned into $\kappa$ pairwise disjoint sets each not in $J$ (and $\left.J_{\mathfrak{a}}^{\text {bd }} \subseteq J\right)$, we can find a sequence $\left\langle\left(\theta_{\Upsilon}, \zeta_{\Upsilon}\right): \Upsilon \in \mathfrak{a}\right\rangle$ such that:

- for each $\Upsilon \in \mathfrak{a}$ we have $\theta_{\Upsilon} \in \mathfrak{a}, \Upsilon \geq \theta_{\Upsilon}, \zeta_{\Upsilon}<\kappa$,

- for each $\theta \in \mathfrak{b}$ and $\zeta<\kappa,\left\{\Upsilon \in \mathfrak{a}: \theta_{\Upsilon}=\theta, \zeta_{\Upsilon}=\zeta\right\} \neq \emptyset \bmod J$.

Now we define a linear order $<_{\text {et }}$ on $\left\{f_{\alpha}: \alpha<\lambda\right\}$ as follows: $f_{\alpha}<_{\text {et }} f_{\beta}$ if and only if for some $\Upsilon \in \mathfrak{a}$ we have

$$
\begin{gathered}
f_{\alpha}\left\lceil(\mathfrak{a} \cap \Upsilon)=f_{\beta}\left\lceil(\mathfrak{a} \cap \Upsilon), \quad f_{\alpha}(\Upsilon) \neq f_{\beta}(\Upsilon) \quad\right. \text { and }\right. \\
f_{\alpha}(\Upsilon)<f_{\beta}(\Upsilon) \Leftrightarrow f_{\alpha}\left\lceil\theta_{\Upsilon} \in F_{\theta_{\Upsilon}, \zeta_{\Upsilon}} .\right.
\end{gathered}
$$

Readily $<_{\text {et }}$ is a linear order on the set $\mathcal{I}=\left\{f_{\alpha}: \alpha<\lambda\right\}$. We are going to show that it is as required (note that in the definition of $f_{\alpha}<_{\text {et }} f_{\beta}$ we have $f_{\alpha}\left\lceil\theta_{\Upsilon}=f_{\beta}\left\lceil\theta_{\Upsilon}\right.\right.$ as $\left.\theta_{\Upsilon} \leq \Upsilon\right)$. Suppose that $\varepsilon(*)<\sigma, u \subseteq \varepsilon(*)$, $v=\varepsilon(*) \backslash u, t_{\alpha}^{\varepsilon}=f_{\gamma(\varepsilon, \alpha)}$ (for $\left.\varepsilon<\varepsilon(*), \alpha<\lambda\right)$ and $\gamma(\varepsilon, \alpha)$ 's are pairwise distinct. For each $\alpha<\lambda$ take $\theta_{\alpha} \in \mathfrak{b}$ such that $\left\{f_{\gamma(\varepsilon, \alpha)}\left\lceil\theta_{\alpha}: \varepsilon<\varepsilon(*)\right\}\right.$ is with no repetitions (possible as $\varepsilon(*)<\sigma \leq \operatorname{cf}(\sup \mathfrak{a}) \leq \kappa$ and $\mathfrak{b} \subseteq \mathfrak{a}$ is unbounded). Since $\lambda$ is regular and $\lambda>\kappa$, we may assume that for each $\alpha<\lambda, \theta_{\alpha}=\theta^{*} \in \mathfrak{b}$. We have $\left|\left\{f_{\alpha} \mid \theta^{*}: \alpha<\lambda\right\}\right| \leq \max \operatorname{pcf}\left(\mathfrak{a} \cap \theta^{*}\right)$ and $\left(\max \operatorname{pcf}\left(\mathfrak{a} \cap \theta^{*}\right)\right)<\sigma<\theta^{*}$ and hence we may assume that for some $\left\langle g_{\varepsilon}: \varepsilon<\varepsilon(*)\right\rangle$,

$$
(\forall \alpha<\lambda)(\forall \varepsilon<\varepsilon(*))\left(f_{\gamma(\varepsilon, \alpha)}\left\lceil\theta^{*}=g_{\varepsilon}\right) .\right.
$$

Let $X=\left\{g_{\varepsilon}: \varepsilon \in u\right\}, Y=\left\{g_{\varepsilon}: \varepsilon \in \varepsilon(*) \backslash u\right\}$ and let $\zeta<\kappa$ be such that $F_{\theta^{*}, \zeta} \cap(X \cup Y)=X$. As in the proof of 3.1 one can show that

$$
\left\{\mu \in \mathfrak{a}:(\forall \xi<\mu)\left(\left|\left\{\alpha<\lambda: g_{\alpha}(\mu)>\xi\right\}\right|=\lambda\right)\right\}=\mathfrak{a} \bmod J
$$

where $g_{\alpha}(\mu)=\min \left\{f_{\gamma(\varepsilon, \alpha)}(\mu): \varepsilon<\varepsilon(*)\right\}$. Thus we can find $\Upsilon \in \mathfrak{a}$ such that $\theta^{*}=\theta_{\Upsilon}, \zeta=\zeta_{\Upsilon}$ and $(\forall \xi<\Upsilon)\left(\left|\left\{\alpha<\lambda: g_{\alpha}(\Upsilon)>\xi\right\}\right|=\lambda\right)$. Next, as in 3.1, we can find $\alpha_{\xi}<\lambda$ (for $\xi<\Upsilon$ ) and $S \in[\Upsilon]^{\Upsilon}$ such that for each $\xi<\Upsilon$ we have $\xi<g_{\alpha_{\xi}}(\Upsilon),(\forall \zeta<\xi)\left(\alpha_{\zeta}<\alpha_{\xi}\right)$ and

$$
(\forall \varepsilon<\varepsilon(*))(\forall \zeta<\xi)\left(f_{\gamma\left(\varepsilon, \alpha_{\zeta}\right)}(\Upsilon)<g_{\alpha_{\xi}}(\Upsilon)\right)
$$

and the sequence $\left\langle\left\langle f_{\gamma\left(\varepsilon, \alpha_{\xi}\right)} \mid \Upsilon: \varepsilon<\varepsilon^{*}\right\rangle: \xi \in S\right\rangle$ is constant. Choose any $\xi_{1}, \xi_{2} \in S, \xi_{1}<\xi_{2}$ and note that for every $\varepsilon<\varepsilon(*)$ we have

$$
f_{\gamma\left(\varepsilon, \alpha_{\xi_{1}}\right)}\left|\Upsilon=f_{\gamma\left(\varepsilon, \alpha_{\xi_{2}}\right)}\right| \Upsilon, \quad f_{\gamma\left(\varepsilon, \alpha_{\xi_{1}}\right)}(\Upsilon)<f_{\gamma\left(\varepsilon, \alpha_{\xi_{2}}\right)}(\Upsilon),
$$

and $f_{\gamma\left(\varepsilon, \alpha_{\xi_{1}}\right)}\left\lceil\theta_{\Upsilon}=g_{\varepsilon}=f_{\gamma\left(\varepsilon, \alpha_{\xi_{2}}\right)} \mid \theta_{\Upsilon}\right.$. Thus $\alpha_{\xi_{1}}<\alpha_{\xi_{2}}$ satisfy the condition given by entangledness for $t_{\alpha}^{\varepsilon}$ 's.

(3) Let $\left\langle f_{\alpha}: \alpha<\lambda\right\rangle$ be as in the proof of part (2). We define a linear order $<_{\text {pet }}$ on $\left\{f_{\alpha}: \alpha<\lambda\right\}$ as follows: $f_{\alpha}<_{\text {pet }} f_{\beta}$ if and only if for some $\Upsilon \in \mathfrak{a}$ we have

$$
f_{\alpha} \uparrow(\mathfrak{a} \cap \Upsilon)=f_{\beta} \uparrow(\mathfrak{a} \cap \Upsilon) \quad \text { and } \quad f_{\alpha}(\Upsilon)<f_{\beta}(\Upsilon)
$$


The rest is even simpler than in the proof of part (2) after defining $<_{\text {et }}$ (remember 1.2(6)).

(4) It is similar to the proof of 3.1(2), noting that if $\bar{f}^{l}=\left\langle f_{\alpha}^{l}: \alpha<\lambda\right\rangle$ is $<_{J}$-increasing cofinal in $\prod \mathfrak{a}$ for $l=1,2, \lambda=\operatorname{cf}(\lambda)$ and $\bar{f}^{1}$ is $\mu$-free then for some $X \in[\lambda]^{\lambda}, \bar{f}^{2}\lceil X$ is $\mu$-free.

Proposition 3.3. (1) Assume that:

(a) $\operatorname{Ens}_{\sigma}\left(\lambda_{i}, \kappa_{i}\right)$ for $i<\delta$,

(b) $\lambda_{i}$ are regular cardinals for $i<\delta,\left\langle\lambda_{i}: i<\delta\right\rangle$ is strictly increasing and $\delta<\lambda_{0}$,

(c) $J$ is a $\sigma$-complete ideal on $\delta$ extending $J_{\delta}^{\mathrm{bd}}$,

(d) $\kappa<T_{J}^{+}\left(\left\langle\kappa_{i}: i<\delta\right\rangle\right)\left(:=\sup \left\{|F|^{+}: F \subseteq \prod_{i<\delta} \kappa_{i}\right.\right.$ and $f \neq g \in$ $\left.\left.F \Rightarrow f \neq \neq_{J} g\right\}\right)$

(e) $\prod_{i<\delta} \lambda_{i} / J$ has the true cofinality $\lambda$ as exemplified by $\left\{f_{\alpha}: \alpha<\lambda\right\}$ and for each $i<\delta, \lambda_{i}>\left|\left\{f_{\alpha} \mid i: \alpha<\lambda\right\}\right|^{<\sigma}$ (if for each $i$, $\max \operatorname{pcf}\left(\left\{\lambda_{j}: j<i\right\}\right)<\lambda_{i}$ then we have such $f_{\alpha}$ 's $)$.

Then $\operatorname{Ens}_{\sigma}(\lambda, \kappa)$.

(2) Assume that in part (1) we omit (d) but in addition we have

(f) for each $i<\delta, \kappa_{i} \geq \mid\left\{f_{\alpha}\lceil i: \alpha<\lambda\} \mid\right.$

[and we have such $f_{\alpha}$ 's e.g. if $\left.\kappa_{i} \geq \max \operatorname{pcf}\left(\left\{\lambda_{j}: j<i\right\}\right)\right]$, or at least $\liminf { }_{J}\left(\kappa_{i}\right)=\sup _{i<\delta} \lambda_{i}$, or

$\left(\mathrm{f}^{\prime}\right) \delta$ can be partitioned $\left({ }^{5}\right)$ into $|\delta| J$-positive sets and for each $i<\delta$ for $J$-almost all $j<\delta$ we have $\kappa_{j} \geq \mid\left\{f_{\alpha}\lceil i: \alpha<\lambda\} \mid\left(\right.\right.$ if $\kappa_{j}$ is strictly increasing this means "every large enough $j$ ").

Then there is a $\sigma$-entangled linear order $\mathcal{I}$ of cardinality $\lambda$.

(3) Assume $\left(\mathrm{f}^{\prime}\right)$ or $(\mathrm{f})+(\mathrm{g})$, where

(g) there is a decreasing sequence $\left\langle B_{\varepsilon}: \varepsilon<\sigma\right\rangle$ of elements of $J^{+}$ with empty intersection.

Then in (2) we can get $\mathcal{I}=\mathcal{I}_{1}+\mathcal{I}_{2}$ such that for any uniform ultrafilter $D$ on $\sigma$ the orders $\left(\mathcal{I}_{1}\right)^{\sigma} / D$ and $\left(\mathcal{I}_{2}\right)^{\sigma} / D$ have isomorphic subsets of cardinality $\lambda$ (see 1.8 for a corollary).

(4) The linear orders (in all parts of 3.3) have exact density $\mu:=$ $\sup _{i<\delta} \lambda_{i}$, and if $\left\langle f_{\alpha}: \alpha<\lambda\right\rangle$ is $\mu$-free even exact density $\left(\mu^{+}, \mu\right)$.

(5) In 3.3(1) we can weaken clause (d) to

(d) ${ }^{-}$for some $F \subseteq \prod_{i<\delta} \kappa_{i},|F|=\kappa$ and for every $F^{\prime} \subseteq F$ of cardinality $<\sigma$ we have $\left\{i<\delta:\left\langle g(i): g \in F^{\prime}\right\rangle\right.$ is with no repetition $\} \in J^{+}$.

\footnotetext{
$\left({ }^{5}\right)$ If $\kappa_{j}$ is non-decreasing then a partition into $\operatorname{cf}(\delta)$ sets suffices.
} 
(6) In 3.3(2) we can replace $\left(\mathrm{f}^{\prime}\right)$ by

$(\oplus) \quad$ there is $h: \delta \rightarrow \delta$ such that $i \geq h(i), \kappa_{i} \geq \mid\left\{f_{\alpha}\lceil h(i): \alpha<\lambda\} \mid\right.$ and for every $i<\delta,\{j<\delta: h(j) \geq i\} \in J^{+}$.

Proof. Similar. (For part (3) look at the proof of 2.3(3).) However, we will give some details.

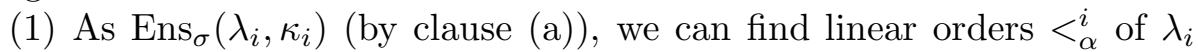
(for $\left.\alpha<\kappa_{i}\right)$ such that the sequence $\left\langle\left(\lambda_{i},<_{\alpha}^{i}\right): \alpha<\kappa_{i}\right\rangle$ is $\sigma$-entangled. By clause (d) we can find $g_{\zeta} \in \prod_{i<\delta} \kappa_{i}($ for $\zeta<\kappa)$ such that

$$
\varepsilon<\zeta<\kappa \Rightarrow\left\{i<\delta: g_{\varepsilon}(i)=g_{\zeta}(i)\right\} \in J .
$$

Now for each $\zeta<\kappa$ we define a linear order $\mathcal{I}_{\zeta}=\left(F,<_{\zeta}^{*}\right)$ with the set of elements $F:=\left\{f_{\alpha}: \alpha<\lambda\right\}$ as follows: $f_{\alpha}<_{\zeta}^{*} f_{\beta}$ if and only if for some $i<\delta$ we have

$$
f_{\alpha}(i) \neq f_{\beta}(i), \quad f_{\alpha}\left\lceil i=f_{\beta}\left\lceil i \quad \text { and } \quad f_{\alpha}(i)<_{g_{\zeta}(i)}^{i} f_{\beta}(i) .\right.\right.
$$

It is easy to check that $<_{\zeta}^{*}$ is a linear order of $F$. For the relevant part of (4), $\mathcal{J} \subset \mathcal{I}_{i},|\mathcal{J}|=\lambda$, so $\mathcal{J}=\left\{f_{\alpha}: \alpha \in X\right\}, X \in[\lambda]^{\lambda}$, so $\mathcal{J}$ 's density is $\leq \mid\left\{f_{\alpha} \mid i: \alpha \in X\right.$ and $\left.i<\delta\right\} \mid=\mu:=\sum_{i<\delta} \lambda_{i}$. As in the proof of 3.1,

$A:=\left\{i<\delta:\left(\exists f \in \prod_{j<i} \lambda_{j}\right)\left(\lambda_{i}=\mid\left\{f_{\alpha}(i): \alpha \in X \& f=f_{\alpha}\lceil i\} \mid\right)\right\}=\delta \bmod J\right.$

and for $i \in A$ let $f_{i}^{*}$ exemplify $i \in A$. If $\mathcal{J}^{\prime} \subseteq \mathcal{J}$ is dense, $\left|\mathcal{J}^{\prime}\right|$ cannot be $<\mu$ as then it is $<\lambda_{i(*)}$ for some $i(*) \in A$ and so for some $\gamma<\lambda_{i(*)}$ for no $\alpha<\lambda$ do we have

$$
f_{\alpha}\left\lceil i(*)=f_{i(*)}^{*} \& \gamma \leq f_{\alpha}(i(*))<\lambda_{i(*)}\right.
$$

thus proving $\mu=\operatorname{dens}(\mathcal{J})$. The part "if $\left\{f_{\alpha}: \alpha<\lambda\right\}$ is $\mu$-free then any $\mathcal{J} \subseteq \mathcal{I}_{j}$ of cardinality $>\mu$ has density $\mu$ (i.e. has exact density $\left(\mu^{+}, \mu\right)$ )" can be proven similarly.

Finally, " $\left\langle\mathcal{I}_{\zeta}: \zeta\langle\kappa\rangle\right.$ is $\sigma$-entangled" is proved as in the proof of 3.1. Assume $u \cup v=\varepsilon(*)<\sigma, u \cap v=\emptyset$, and $\zeta_{\varepsilon}<\kappa$ for $\varepsilon<\varepsilon(*)$ are pairwise distinct. Now

$$
A:=\left\{i<\delta:\left\langle g_{\varepsilon}(i): \varepsilon<\varepsilon(*)\right\rangle \text { are pairwise distinct }\right\} \neq \emptyset \bmod J
$$

(as $J$ is $\sigma$-complete, $(\mathrm{d}) \Rightarrow(\mathrm{d})^{-}$). We continue as in the proof of 3.1 (only with $A$ as here) and using the fact that $\left\langle\left(\lambda_{i},<_{\alpha}^{i}\right): \alpha<\kappa_{i}\right\rangle$ is $\sigma$-entangled.

(2) First we assume clause (f). As $\operatorname{Ens}_{\sigma}\left(\lambda_{i}, \kappa_{i}\right)$ and $\kappa_{i} \geq\left|\Pi_{i}\right|$ where $\Pi_{i}:=\left\{f_{\alpha} \mid i: \alpha<\lambda\right\}$, we can find linear orders $<_{\eta}^{i}$ of $\lambda_{i}$ (for $\eta \in \Pi_{i}$ ) such that $\left\langle\left(\lambda_{i},<_{\eta}^{i}\right): \eta \in \Pi_{i}\right\rangle$ is $\sigma$-entangled. We define the linear order $<^{*}$ of $F:=\left\{f_{\alpha}: \alpha<\lambda\right\}$ as follows: $f_{\alpha}<^{*} f_{\beta}$ if and only if for some $i<\delta$ we have

$$
f_{\alpha}(i) \neq f_{\beta}(i), \quad f_{\alpha}\left\lceil i=f_{\beta}\left\lceil i, \quad \text { and } \quad f_{\alpha}(i)<_{f_{\alpha}\lceil i}^{i} f_{\beta}(i) .\right.\right.
$$

The rest is as in $3.3(1)$. 
Next we assume clause $\left(\mathrm{f}^{\prime}\right)$ instead of (f). So let $\left\langle A_{i}: i<\delta\right\rangle$ be a partition of $\delta$ with every $A_{j}$ in $J^{+}$and so necessarily

$$
A_{i}^{\prime}:=\left\{j \in A_{i}: \kappa_{j} \geq\left|\Pi_{i}\right|\right\}=A_{i} \bmod J .
$$

Then we can choose $\left({ }^{6}\right)$ a function $h$ such that

$$
\begin{gathered}
h: \delta \rightarrow \delta, h(i) \leq i, \kappa_{i} \geq\left|\Pi_{h(i)}\right| \text { and for every } i<\delta \text { we have } \\
\{j<\delta: h(j) \geq i\} \in J^{+} .
\end{gathered}
$$

Let $\left\langle\left(\lambda_{i},<_{\eta}^{i}\right): \eta \in \Pi_{h(i)}\right\rangle$ be a $\sigma$-entangled sequence of linear orders. Now we define a linear order on $F:=\left\{f_{\alpha}: \alpha<\lambda\right\}: f_{\alpha}<^{*} f_{\beta}$ if and only if for some $i<\delta$ we have

$$
f_{\alpha}(i) \neq f_{\beta}(i), \quad f_{\alpha}\left\lceil i=f_{\beta}\left\lceil i, \quad \text { and } \quad f_{\alpha}(i)<_{f_{\alpha}\lceil h(i)}^{i} f_{\beta}(i) .\right.\right.
$$

(3) Without loss of generality, for each $\xi<\sigma+\sigma$ the set $J_{\xi}:=\{\alpha<\lambda$ : $\left.f_{\alpha}(0)=\xi\right\}$ has cardinality $\lambda$. Let $F=\left\{f_{\alpha}: \alpha<\lambda \& f_{\alpha}(0)<\sigma+\sigma\right\}$. We can find $B_{\varepsilon} \in J^{+}$(for $\varepsilon<\sigma$ ), decreasing with $\varepsilon$ and such that $\bigcap_{\varepsilon<\sigma} B_{\varepsilon}=\emptyset$, and in the proof from $\left(\mathrm{f}^{\prime}\right)$ replace $(\otimes)$ by

$h: \delta \rightarrow \delta, h(i) \leq i, \kappa_{i} \geq\left|\Pi_{h(i)}\right|$ and for every $i<\delta, \xi<\sigma+\sigma$ we have

$$
\left\{j \in B_{\xi}: h(j) \geq i\right\} \in J^{+},
$$

and define $<^{*}$ by $f_{\alpha}<^{*} f_{\beta}$ if and only if for some $i<\delta$ we have

$$
\begin{gathered}
f_{\alpha}(i) \neq f_{\beta}(i), \quad f_{\alpha}\left\lceil i=f_{\beta}\lceil i,\right. \\
{\left[i=0 \Rightarrow f_{\alpha}(0)<f_{\beta}(0)\right], \quad\left[0<i \in B_{f_{\alpha}(0)} \Rightarrow f_{\alpha}(i)<i_{f_{\alpha}\lceil h(i)} f_{\beta}(i)\right],} \\
{\left[0<i \notin B_{f_{\alpha}(0)} \Rightarrow f_{\alpha}(i)<f_{\beta}(i)\right] .}
\end{gathered}
$$

The rest is as before (we can replace $\sigma$ by other cardinals $\geq \sigma$ but $\leq \lambda_{0}$ ).

(4) For 3.3(1) see in its proof, other cases similar.

(5) Really included in the proof of 3.3(1).

(6) Really included in the proof of $3.3(2)$. $\mathbf{- 3}_{3.3}$

Proposition 3.4. (1) Assume that:

(a) $\operatorname{Ens}_{\sigma}\left(\lambda_{i}, \mu_{i}, \kappa_{i}\right)$ for $i<\delta$,

(b) $\left\langle\lambda_{i}: i<\delta\right\rangle$ is a strictly increasing sequence of regular cardinals, $2^{|\delta|}<\lambda_{0}$

(c) $J$ is a $\sigma$-complete ideal on $\delta$ extending $J_{\delta}^{\mathrm{bd}}$,

(d) $\kappa<T_{J}^{+}\left(\left\langle\kappa_{i}: i<\delta\right\rangle\right), \sum_{i<\delta} \lambda_{i} \leq \mu<\lambda, \mu=\operatorname{cf}(\mu)$ and

$$
(\forall \alpha<\mu)\left(|\alpha|^{<\delta}<\mu\right)
$$

$\left({ }^{6}\right)$ Actually we can replace the assumption (g) (in 3.3(3)) by the existence of such an $h$. 
(e) $F=\left\{f_{\alpha}: \alpha<\lambda\right\} \subseteq \prod_{i<\delta} \lambda_{i}, f_{\alpha} \neq_{J} f_{\beta}$ for $\alpha \neq \beta$, and

$$
\mid\left\{\left.f_{\alpha}\lceil i: \alpha<\lambda\}\right|^{<\sigma}<\mu_{i},\right.
$$

(f) if $\mu_{i}^{\prime}=\operatorname{cf}\left(\mu_{i}^{\prime}\right)<\mu_{i}$ and $A \in J^{+}$then $\operatorname{tcf}\left(\prod_{i \in A} \mu_{i}^{\prime} / J\right)=\mu$ is impossible,

(g) $(\forall \alpha<\mu)\left(|\alpha|^{<\sigma}<\mu\right)$.

Then $\operatorname{Ens}_{\sigma}(\lambda, \mu, \kappa)$.

(2) Assume in addition

or at least

(h) $\kappa_{i} \geq\left|\left\{f_{\alpha} \mid i: \alpha<\lambda\right\}\right|$,

$\left(\mathrm{h}^{\prime}\right) \operatorname{cf}(\delta)=\omega$ and $\liminf _{J} \kappa_{i}=\sup _{i<\delta} \lambda_{i}$,

or at least

$\left(\mathrm{h}^{\prime \prime}\right)$ there is $h: \delta \rightarrow \delta$ such that $i \geq h(i), \kappa_{i} \geq \mid\left\{f_{\alpha}\lceil h(i): \alpha<\lambda\}\right.$ and $\delta=\lim \sup _{i<\delta} h(i)$.

Then there is a $(\mu, \sigma)$-entangled linear order $\mathcal{I}$ of cardinality $\lambda$.

(3) Suppose also that

(i) we can partition $\delta$ into $\sigma$ sets from $J^{+}$(or clause (g) from 3.3(3) holds).

Then we can get: for any uniform ultrafilter $D$ on $\sigma, \mathcal{I}^{\sigma} / D$ has two isomorphic subsets with disjoint convex hulls of cardinality $\lambda$.

Pr o of. Similar proof but for the reader's convenience we present some details.

(1) We repeat the proof of 3.3(1) up to proving entangledness. To show " $\left\langle\mathcal{I}_{\zeta}: \zeta<\kappa\right\rangle$ is $(\mu, \sigma)$-entangled" suppose that $u \cup v=\varepsilon(*)<\sigma, u \cap v=\emptyset$ and $\left\langle\zeta_{\varepsilon}: \varepsilon<\varepsilon(*)\right\rangle$ is a sequence of pairwise distinct ordinals $<\kappa$ and $\langle\gamma(\beta, \varepsilon): \beta<\mu, \varepsilon<\varepsilon(*)\rangle \subseteq \lambda$ is such that

$$
(\forall \varepsilon<\varepsilon(*))\left(\forall \beta_{1}<\beta_{2}<\lambda\right)\left(\gamma\left(\beta_{1}, \varepsilon\right) \neq \gamma\left(\beta_{2}, \varepsilon\right)\right) .
$$

We want to find $\beta_{1}<\beta_{2}<\mu$ such that

$$
(\forall \varepsilon<\varepsilon(*))\left(\gamma\left(\beta_{1}, \varepsilon\right)<_{\zeta_{\varepsilon}} \gamma\left(\beta_{2}, \varepsilon\right) \Leftrightarrow \varepsilon \in u\right) .
$$

Claim 3.4.1. Assume that:

$(\alpha)\left\langle\lambda_{i}: i<\delta\right\rangle$ is a strictly increasing sequence of regular cardinals,

( $\beta) J$ is a $\sigma$-complete ideal on $\delta$ extending $J_{\delta}^{\mathrm{bd}}$,

$(\gamma)$ a sequence $\bar{f}=\left\langle f_{\alpha}: \alpha<\lambda\right\rangle \subseteq \prod_{i<\delta} \lambda_{i}$ is $<_{J}$-increasing,

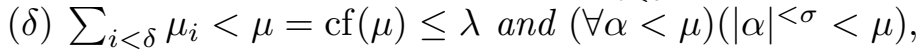

( $\varepsilon$ ) either (i) $2^{|\delta|}<\lambda_{0}$, or (ii) $\bar{f}$ is $\mu$-free,

(ל) if $\mu_{i}^{\prime}<\mu_{i}$ for $i<\delta$ and $A \in J^{+}$then $\operatorname{tcf}\left(\prod_{i \in A} \mu_{i} / J\right)=\mu$ is impossible, 
$(\theta)$ a sequence $\langle\gamma(\beta, \varepsilon): \beta<\mu, \varepsilon<\varepsilon(*)<\sigma\rangle$ of ordinals $<\lambda$ satisfies

$$
\left(\beta_{1}, \varepsilon_{1}\right) \neq\left(\beta_{2}, \varepsilon_{2}\right) \Rightarrow \gamma\left(\beta_{1}, \varepsilon_{1}\right) \neq \gamma\left(\beta_{2}, \varepsilon_{2}\right) .
$$

Then there are a set $X \in[\mu]^{\mu}$ and a sequence $\left\langle h_{\varepsilon}: \varepsilon<\varepsilon(*)\right\rangle \subseteq \prod_{i<\delta} \lambda_{i}$ such that:

(a) for each $\varepsilon<\varepsilon(*)$ the sequence $\langle\gamma(\beta, \varepsilon): \beta \in X\rangle$ is strictly increasing,

(b) $(\forall \beta \in X)(\forall \varepsilon<\varepsilon(*))\left(f_{\gamma(\beta, \varepsilon)}<{ }_{J} h_{\varepsilon}\right)$,

(c) $\left\langle h_{\varepsilon}(i): i<\delta\right\rangle$ is the $<_{J}$-eub of $\left\langle f_{\gamma(\beta, \varepsilon)}: \beta \in X\right\rangle$,

(d) $B^{*}=\left\{i<\delta:(\forall \varepsilon<\varepsilon(*))\left(\operatorname{cf}\left(h_{\varepsilon}(i)\right) \geq \mu_{i}\right)\right\}=\delta \bmod J$.

Proof. Since $(\forall \alpha<\mu)\left(|\alpha|^{<\sigma}<\mu\right)$ and $\operatorname{cf}(\mu)=\mu$ we know that for some $X \in[\mu]^{\mu}$ we have

$(\forall \varepsilon<\varepsilon(*))$ (the sequence $\langle\gamma(\beta, \varepsilon): \beta \in X\rangle$ is strictly increasing).

[Why? For $\beta<\mu, \varepsilon<\varepsilon(*)$ define $f(\beta, \varepsilon)$ as follows: if there exists $\delta<\beta$ such that $\gamma(\delta, \varepsilon)>\gamma(\beta, \varepsilon)$ and $[\gamma(\beta, \varepsilon), \gamma(\delta, \varepsilon)] \cap\{\gamma(\alpha, \varepsilon): \alpha<\beta\}=\emptyset$ then $f(\beta, \varepsilon)$ is this unique $\delta$, otherwise $f(\beta, \varepsilon)=-1$. By Fodor's lemma, there is a stationary set $S \subseteq \mu$ such that $\sup f[S \times \varepsilon(*)]<\mu$. Since $|\alpha|^{\varepsilon(*)}<\mu$ for $\alpha<\mu$, on a stationary set $X \subseteq S$ the sequence $\langle f(\alpha, \varepsilon): \varepsilon<\varepsilon(*)\rangle$ does not depend on $\alpha$. This $X$ is as required.]

By renaming we may assume that $X=\mu$. Consequently, for each $\varepsilon<$ $\varepsilon(*)$ the sequence $\left\langle f_{\gamma(\beta, \varepsilon)}: \beta<\mu\right\rangle$ is $<_{J}$-increasing. Since either $\mu=\operatorname{cf}(\mu)>$ $2^{|\delta|}$ or the sequence is $\mu$-free, we may use $[$ Sh $355, \S 1]$ to conclude that it has a $<{ }_{J}$-eub; call it $h_{\varepsilon}$. We may assume that, for each $i<\delta, h_{\varepsilon}(i)$ is a limit ordinal. Since $h_{\varepsilon}<_{J}\left\langle\lambda_{i}: i<\delta\right\rangle$, without loss of generality we have $(\forall i<\delta)\left(h_{\varepsilon}(i)<\lambda_{i}\right)$. Also $\mu=\operatorname{tcf}\left(\prod_{i<\delta} \operatorname{cf}\left(h_{\varepsilon}(i)\right) / J\right)$ and $\operatorname{cf}\left(h_{\varepsilon}(i)\right) \leq$ $h_{\varepsilon}(i)<\lambda_{i}$, so by the assumption $(\varepsilon)$ we have

$$
\left\{i<\delta: \operatorname{cf}\left(h_{\varepsilon}(i)\right)<\mu_{i} \text { or } \max \operatorname{pcf}\left(\left\{\lambda_{j}: j<i\right\}<\operatorname{cf}\left(h_{\varepsilon}(i)\right)\right\}\right)=\emptyset \bmod J
$$

Since $J$ is $\sigma$-complete we conclude

$$
B^{*}=\left\{i<\delta:(\forall \varepsilon<\varepsilon(*))\left(\operatorname{cf}\left(h_{\varepsilon}(i)\right) \geq \mu_{i}\right)\right\}=\delta \bmod J,
$$

finishing the proof of the claim. $\mathbf{m}_{3.4 .1}$

Note that we may assume that for some $i^{\otimes}<\delta$, for every $\beta<\mu$ the sequence $\left\langle f_{\gamma(\beta, \varepsilon)}\left\lceil i^{\otimes}: \varepsilon<\varepsilon(*)\right\rangle\right.$ is with no repetition and does not depend on $\beta$. Now we may apply 3.4.1 to find $X \in[\mu]^{\mu}$ and $\left\langle h_{\varepsilon}: \varepsilon\langle\varepsilon(*)\rangle\right.$ as there. We shall continue as in the proof of 3.1 with some changes, however. We let

$$
\begin{array}{r}
G_{i}=\left\{\left\langle f_{\gamma(\beta, \varepsilon)}(i): \varepsilon<\varepsilon(*)\right\rangle: \beta<\mu\right\} \subseteq \prod_{\varepsilon<\varepsilon(*)} h_{\varepsilon}(i), \\
B=\left\{i \in B^{*}: \text { for each }\left\langle\xi_{\varepsilon}: \varepsilon<\varepsilon(*)\right\rangle \in \prod_{\varepsilon<\varepsilon(*)} h_{\varepsilon}(i)\right. \\
\text { there are } \mu \text { ordinals } \beta<\mu \text { such that }
\end{array}
$$$$
\left.(\forall \varepsilon<\varepsilon(*))\left(\xi_{\varepsilon}<f_{\gamma(\beta, \varepsilon)}(i)\right)\right\} \text {. }
$$ 
We have to show the following.

Claim 3.4.2. $B=\delta \bmod J$.

Proof. Otherwise, as $B^{*}=\delta \bmod J$, necessarily $B^{*} \backslash B \neq \emptyset \bmod J$. For each $i \in B^{*} \backslash B$ choose a sequence $\left\langle\xi_{\varepsilon}^{i}: \varepsilon<\varepsilon(*)\right\rangle \in \prod_{\varepsilon<\varepsilon(*)} h_{\varepsilon}(i)$ and an ordinal $\beta_{i}<\mu$ exemplifying $i \notin B$. Thus if $i \in B^{*} \backslash B$ and $\beta \in\left[\beta_{i}, \mu\right)$ then $(\exists \varepsilon<\varepsilon(*))\left(\xi_{\varepsilon}^{i} \geq f_{\gamma(\beta, \varepsilon)}(i)\right)$.

For $\varepsilon<\varepsilon(*)$ define a function $h^{\varepsilon} \in \prod_{i<\delta} h_{\varepsilon}(i)$ by

$$
h^{\varepsilon}(i)= \begin{cases}\xi_{\varepsilon}^{i}+1 & \text { if } i \in B^{*} \backslash B, \\ 0 & \text { if } i \in \delta \backslash B^{*} \text { or } i \in B .\end{cases}
$$

Now, for each $\varepsilon$, for every sufficiently large $\beta<\mu$ we have $h^{\varepsilon}<_{J} f_{\gamma(\beta, \varepsilon)}$. Consequently, we find $\beta^{*}<\mu$ such that

$$
\varepsilon<\varepsilon(*) \& \beta \in\left[\beta^{*}, \mu\right) \Rightarrow h^{\varepsilon}<_{J} f_{\gamma(\beta, \varepsilon)} .
$$

But the ideal $J$ is $\sigma$-complete, so for each $\beta \in\left[\beta^{*}, \mu\right)$,

$$
B_{\beta}:=\left\{i<\delta:(\forall \varepsilon<\varepsilon(*))\left(h^{\varepsilon}(i)<f_{\gamma(\beta, \varepsilon)}(i)\right)\right\}=\delta \bmod J .
$$

Now we may take $\beta \in\left[\beta^{*}, \mu\right)$ and then choose $i \in B_{\beta} \cap\left(B^{*} \backslash B\right)$ and get a contradiction as in the proof of 3.1.1. 3.4 .2

Remember that

$$
\left|\left\{\left\langle f_{\gamma(\beta, \varepsilon)} \mid i: \varepsilon<\varepsilon(*)\right\rangle: \beta<\mu\right\}\right| \leq\left|\left\{f_{\alpha} \mid i: \alpha<\lambda\right\}\right|^{<\sigma}<\mu_{i}
$$

(not just $<\lambda_{i}$ ); see clause (e) of the assumptions of 3.4(1). Hence there is $\beta^{\otimes}<\mu$ such that

$$
\begin{aligned}
(\forall \delta \in & {\left.\left[\beta^{\otimes}, \mu\right)\right)(\forall j<\delta) } \\
& \left(\mid\left\{\beta:\left\langle f_{\gamma(\delta, \varepsilon)} \mid j: \varepsilon<\varepsilon(*)\right\rangle=\left\langle f_{\gamma(\beta, \varepsilon)}\lceil j: \varepsilon<\varepsilon(*)\rangle, \beta \in\left[\beta^{\otimes}, \mu\right)\right\} \mid=\mu\right) .\right.
\end{aligned}
$$

For each $i \in B$ (defined above) we know that $(\forall \varepsilon<\varepsilon(*))\left(\operatorname{cf}\left(h_{\varepsilon}(i)\right) \geq \mu_{i}\right)$, and hence $\prod_{\varepsilon<\varepsilon(*)} h_{\varepsilon}(i)$ is $\mu_{i}$-directed and the set

$$
\left\{\left\langle f_{\gamma(\beta, \varepsilon)}(i): \varepsilon<\varepsilon(*)\right\rangle: \beta \in\left[\beta^{\otimes}, \mu\right)\right\}
$$

is cofinal in $\prod_{\varepsilon<\varepsilon(*)} h_{\varepsilon}(i)$. Putting these together, there are $\nu_{\varepsilon}^{i} \in \prod_{j<i} \lambda_{j}$ (for $\varepsilon<\varepsilon(*)$ ) such that for every $\left\langle\xi_{\varepsilon}: \varepsilon<\varepsilon(*)\right\rangle \in \prod_{\varepsilon<\varepsilon(*)} h_{\varepsilon}(i)$, for some $\beta \in\left[\beta^{\otimes}, \mu\right)$ we have

$$
(\forall \varepsilon<\varepsilon(*))\left(f_{\gamma(\beta, \varepsilon)}\left\lceil i=\nu_{\varepsilon}^{i} \& \xi_{\varepsilon}<f_{\gamma(\beta, \varepsilon)}(i)\right) .\right.
$$

Now take any $i \in B$ with $i>i^{\otimes}$ such that the sequence $\left\langle g_{\zeta_{\varepsilon}}(i): \varepsilon<\varepsilon(*)\right\rangle$ is with no repetition. Again, as $\prod_{\varepsilon<\varepsilon(*)} h_{\varepsilon}(i)$ is $\mu_{i}$-directed we can choose by induction on $\alpha<\mu_{i}$ a sequence $\left\langle\beta_{\alpha}: \alpha<\mu_{i}\right\rangle \subseteq \mu_{i}$ such that for each $\alpha<\mu_{i}, \sup \left\{\beta_{\alpha^{\prime}}: \alpha^{\prime}<\alpha\right\}<\beta_{\alpha}$ and

$$
\left(\forall \alpha^{\prime}<\alpha\right)(\forall \varepsilon<\varepsilon(*))\left(f_{\gamma\left(\beta_{\alpha^{\prime}}, \varepsilon\right)}(i)<f_{\gamma\left(\beta_{\alpha}, \varepsilon\right)}(i)\right) .
$$


Now recall that the sequence $\left\langle<_{\zeta}^{i}: \zeta<\kappa_{i}\right\rangle$ exemplifies $\operatorname{Ens}_{\sigma}\left(\lambda_{i}, \mu_{i}, \kappa_{i}\right)$. So we apply this to $\left\langle g_{\zeta_{\varepsilon}}(i): \varepsilon<\varepsilon(*)\right\rangle$ and $\left\langle\left\langle f_{\gamma\left(\beta_{\alpha}, \varepsilon\right)}: \varepsilon<\varepsilon(*)\right\rangle: \alpha<\mu\right\rangle$, and we find $\alpha_{1}<\alpha_{2}<\mu$ such that

$$
\begin{aligned}
& \varepsilon \in u \Rightarrow f_{\gamma\left(\alpha_{1}, \varepsilon\right)}(i)<_{g_{\zeta_{\varepsilon}}(i)}^{i} f_{\gamma\left(\alpha_{2}, \varepsilon\right)}(i), \\
& \varepsilon \in v \Rightarrow f_{\gamma\left(\alpha_{2}, \varepsilon\right)}(i)<_{g_{\zeta_{\varepsilon}}(i)}^{i} f_{\gamma\left(\alpha_{1}, \varepsilon\right)}(i),
\end{aligned}
$$

so we are done.

(2) The proof is exactly like that of 3.4(1) except for two points. First we have to define a linear order $\mathcal{I}$ (rather than $\mathcal{I}_{\zeta}$ for $\zeta<\kappa$ ). Assuming that clause (h) of the assumptions holds, for each $i$ we can find linear orders $<_{\eta}^{i}$ on $\lambda_{i}$ (for $\eta \in T_{i}=\left\{f_{\alpha}\lceil i: \alpha<\lambda\}\right.$ ) such that $\left\langle\left(\lambda_{i},<_{\eta}^{i}\right): \eta \in T_{i}\right\rangle$ is a $\left(\mu_{i}, \sigma\right)$-entangled sequence of linear orders. This does not affect the proof except at the very end when we use the entangledness. Similarly assuming $\left(\mathrm{h}^{\prime}\right)$ or $\left(\mathrm{h}^{\prime \prime}\right)$.

(3) Combine the proofs above. $\mathbf{-}_{3.4}$

Remark 3.5. (1) We can also vary $\sigma$.

(2) The " $2^{|\delta|}<\lambda_{0}$ " rather than just " $|\delta|<\lambda_{0}$ " is needed just to have $<_{J}$-eub (to use the fact that "if $\mu_{i}=\operatorname{cf}\left(\mu_{i}\right)<\mu_{i} \ldots$ ".) so if $\left\{f_{\alpha}: \alpha<\lambda\right\}$ is $\left(\sum_{i<\delta} \lambda_{i}\right)$-free, we can weaken " $2^{|\delta|}<\lambda_{0}$ " to " $|\delta|<\lambda_{0}$ ".

(3) Instead of $T_{J}^{+}\left(\left\langle\kappa_{i}: i<\delta\right\rangle\right)$ we may use any $\chi=|G|$ with $G \subseteq \prod_{i<\delta} \kappa_{i}$ such that for every sequence $\left\langle g_{\varepsilon}: \varepsilon<\varepsilon(*)\right\rangle$ of distinct elements of $G$ the set

$$
\left\{i<\delta:\left\langle g_{\varepsilon}(i): \varepsilon<\varepsilon(*)\right\rangle \text { is with no repetition }\right\}
$$

belongs to $J^{+}$. But then in 3.4(3) we have to change (i).

Proposition 3.6. (1) Suppose $\mu=\mu^{<\sigma}$. Then the set

$$
\left\{\delta<\mu^{+4}: \text { if } \operatorname{cf}(\delta) \geq \sigma \text { then } \operatorname{Ens}_{\sigma}\left(\mu^{+\delta+1}, 2^{\operatorname{cf}(\delta)}\right) \text { or } \operatorname{Ens}_{\sigma}\left(\mu^{+\delta+1}, 2^{\mu^{+}}\right)\right\}
$$

contains a club of $\mu^{+4}$.

(2) If in addition $2^{\mu} \geq \aleph_{\mu^{+4}}$ (or $\left.2^{\mu^{+}} \geq \aleph_{\mu^{+4}}\right)$ then the set

$$
\left\{\delta \leq \mu^{+4}: \text { if } \operatorname{cf}(\delta) \geq \mu\left(\text { or } \operatorname{cf}(\delta) \geq \mu^{+}\right)\right. \text {then }
$$

$$
\text { there is a } \left.\sigma \text {-entangled linear order in } \mu^{+\delta+1}\right\}
$$

contains a club of $\mu^{+4}$ and $\mu^{+4}$ itself. (We can weaken the assumptions.)

(3) We can add in part (2) the conditions needed for 1.7. Also in parts (1), (2) the exact density of the linear orders is $\mu^{+\delta}$ provided $\operatorname{cf}(\delta) \leq \mu^{+}$.

Proof. (1) By [Sh 400, §4], for some club $C$ of $\mu^{+4}$,

$$
\alpha<\delta \in C \Rightarrow \mu^{+\delta}>\operatorname{cov}\left(\mu^{+\alpha}, \mu^{+}, \mu^{+}, 2\right)
$$

and hence, if $\operatorname{cf}(\delta) \geq \sigma$ and $\delta \in C$ then $\left(\mu^{+\delta}\right)^{<\sigma}=\mu^{+\delta}$.

Let $\delta$ be an accumulation point of $C$ of cofinality $\geq \aleph_{1}$ and let $A \subseteq \delta$ be a closed unbounded set such that $\prod_{\alpha \in A} \mu^{+\alpha+1} / J_{A}^{\text {bd }}$ has the true cofinality 
$\mu^{+\delta+1}$ and $\operatorname{otp}(A)=\operatorname{cf}(\delta)$ (exists by [Sh 355, 2.1]). Now for $\beta \in A$ we have $\max \operatorname{pcf}\left(\left\{\mu^{+\alpha+1}: \alpha \in \beta \cap A\right\}\right)<\mu^{+\delta}<\mu^{+\delta+1}$ if $\operatorname{cf}(\delta) \leq \mu$ by $(*)$ (and $[$ Sh $355,5.4])$. Hence for some closed unbounded set $B \subseteq A$ we have

$$
\alpha \in \operatorname{nacc}(B) \Rightarrow \operatorname{cov}\left(\mu^{+\sup (A \cap \alpha)+1}, \mu^{+}, \mu^{+}, 2\right)<\mu^{+\alpha} .
$$

Hence, if $\alpha \in \operatorname{nacc}(B)$ then $\max \operatorname{pcf}\left(\left\{\mu^{+(\beta+1)}: \beta \in B \cap \alpha\right\}\right)<\mu^{+\alpha+1}$. Without loss of generality $\operatorname{otp}(B)=\operatorname{cf}(\delta)$ and $B=A$. Now if $\sigma \leq \operatorname{cf}(\delta)$ $\leq \mu^{+}$then we may apply 3.1 to $\left\{\mu^{+\alpha+1}: \alpha \in \operatorname{nacc}(A)\right\}$ (for $\bar{\lambda}$ ) and get $\operatorname{Ens}_{\sigma}\left(\mu^{+\delta+1}, 2^{\operatorname{cf}(\delta)}\right)$. We still have to deal with the case $\operatorname{cf}(\delta)>\mu^{+}$. We try to choose by induction on $i$ ordinals $\alpha_{i} \in A \backslash \bigcup_{j<i}\left(\alpha_{j}+1\right)$ such that

$$
\mu^{+\alpha_{i}}>\max \operatorname{pcf}\left(\left\{\mu^{+\alpha_{j}}: j<i\right\}\right)<\sigma, \quad \operatorname{cf}\left(\alpha_{i}\right)=\mu^{+} .
$$

For some $\gamma$ the ordinal $\alpha_{i}$ is defined if and only if $i<\gamma$. Necessarily $\gamma$ is limit and by $(*)+[$ Sh $371,1.1]$ we have $\operatorname{cf}(\gamma) \geq \mu^{+}$. Now for each $i<\gamma, \operatorname{Ens}_{\sigma}\left(\mu^{+\alpha_{i}+1}, 2^{\mu^{+}}\right)$holds by the part we have already proved (as $\left.\operatorname{cf}\left(\alpha_{i}\right)=\mu^{+}\right)$. So we may apply 3.3(1).

(2) Let $C$ be as in the proof of part (1) and exemplifying its conclusion. If $\delta=\sup (C \cap \delta)<\mu^{+4}$ and $\operatorname{cf}(\delta) \leq \mu^{+}$and $2^{\mathrm{cf}(\delta)} \geq \mu^{+\delta}$ then we can apply $3.2(1)$ (and the proof of part (1)). So we are left with the case $\operatorname{cf}(\delta)>\mu^{+}$. Now we can repeat the proof of part (1) for the case $\operatorname{cf}(\delta)>\mu^{+}$. Choose $A$ as there and also $\alpha_{i} \in A$, but demand in addition $\operatorname{Ens}_{\sigma}\left(\mu^{+(\delta+1)}, 2^{\mu^{+}}\right)$, $\alpha_{i} \in \operatorname{acc}(C)$ and $\operatorname{cf}\left(\alpha_{i}\right)=\mu^{+}$, hence $\operatorname{Ens}_{\sigma}\left(\mu^{+(\delta+1)}, 2^{\mu^{+}}\right)$. In the end apply $3.3(2)$ to $\left\langle\mu^{+\left(\alpha_{i}+1\right)}: i<\gamma\right\rangle$.

(3) Similar to the proof of 3.3(4), 3.3(5). $\mathbf{m}_{3.6}$

Conclusion 3.7. Assume $\sigma \geq \aleph_{0}$. Then for arbitrarily large cardinals $\lambda$ there are $\sigma$-entangled linear orders of cardinality $\lambda^{+}$.

Proof. Let $\chi>\sigma$ be given. We choose by induction on $i<\sigma$ regular cardinals $\lambda_{i}>\chi$ such that $\operatorname{Ens}_{\sigma}\left(\lambda_{i}, \aleph_{0}+\prod_{j<i} \lambda_{j}\right)$ holds and $\lambda_{i}>\prod_{j<i} \lambda_{j}$. The inductive step is done by 3.6. Now for some $\sigma$-complete ideal $I$ on $\sigma$ extending $J_{\sigma}^{\mathrm{bd}}, \prod_{j<i} \lambda_{j} / I$ has a true cofinality, say $\lambda$. By 3.3 there is a $\sigma$-entangled linear order of cardinality $\lambda$, so if $\lambda$ is a successor cardinal we are done (as $\lambda>\chi)$. If not, necessarily $\lambda$ is inaccessible and letting $\mu=\sum_{j<i} \lambda_{j}$ we clearly have $\mu=\mu^{<\sigma}<\lambda \leq \mu^{\sigma}$. Now we use 3.6(2) to find $\lambda_{i} \in\left(\mu, \aleph_{\mu^{+4}}\right)$ such that there is an entangled linear order in $\lambda_{i}^{+}$, so in any case we are done. $\mathbf{\square}_{3.7}$

4. Boolean algebras with neither pies nor chains. Let us recall the following definition.

Definition 4.1. Let $B$ be a Boolean algebra.

(a) We say that a set $Y \subseteq B$ is a chain of $B$ if

$$
(\forall x, y \in Y)\left(x \neq t \Rightarrow x<_{B} y \text { or } y<_{B} x\right) .
$$


(b) We say that a set $Y \subseteq B$ is a pie of $B$ if

$$
(\forall x, y \in Y)(x \neq y \Rightarrow x \not \leq y \text { and } y \not \leq x) \text {. }
$$

(c) $\pi(B)$, the (algebraic) density of $B$, is

$\min \left\{|X|: X \subseteq B \backslash\{0\}\right.$ and $\left.(\forall y)(\exists x \in X)\left(0<_{B} y \in B \Rightarrow x \leq_{B} y\right)\right\}$.

Lemma 4.2. (1) Suppose that:

(a) $\left\langle\lambda_{i}: i<\delta\right\rangle$ is a strictly increasing sequence of regular cardinals and $\lambda$ is a regular cardinal,

(b) $J$ is a $\sigma$-complete ideal on $\delta$ extending $J_{\delta}^{\mathrm{bd}}$,

(c) $\left\langle f_{\alpha}: \alpha<\lambda\right\rangle$ is a $<_{J}$-increasing sequence of functions from $\prod_{i<\delta} \lambda_{i}$, cofinal in $\left(\prod_{i<\delta} \lambda_{i},<_{J}\right)$,

(d) for every $i<\delta,\left|\left\{f_{\alpha} \mid i: \alpha<\lambda\right\}\right|^{<\sigma}<\lambda_{i}$,

(e) $\left\langle A_{\zeta}: \zeta<\kappa\right\rangle \subseteq \delta$ is a sequence of pairwise disjoint sets such that for every $B \in J$ and $\zeta<\kappa$ there is $i \in \delta$ such that $\{2 i, 2 i+1\} \subseteq$ $A_{\zeta} \backslash B$

(f) $2^{\kappa} \geq \mu:=\sup _{i<\delta} \lambda_{i}$ and $\kappa=\kappa^{<\sigma}($ so $\kappa \leq|\delta| \leq \mu)$ and $\operatorname{cf}(\delta) \leq \kappa$.

Then there is a Boolean algebra $B$ of cardinality $\lambda$ such that:

$(\oplus)_{\lambda}^{B} \quad B$ has neither a chain of cardinality $\lambda$ nor a pie of cardinality $\lambda$ (i.e. $\operatorname{inc}^{+}(B) \leq \lambda$ and Length $\left.{ }^{+}(B) \leq \lambda\right)$.

$(\otimes)_{\mu}^{B} \quad B$ has algebraic density $\pi(B)=\mu($ in fact, for $a \in B \backslash\{0\}, \pi(B\lceil a)$ $=\mu)$.

This also applies to the $\sigma$-complete algebra which $B$ generates, provided $(\forall \alpha<\lambda)\left(|\alpha|^{<\sigma}<\lambda\right)$.

(2) Suppose $2 \leq n^{*} \leq \omega$ and that in part (1) we replace (e) by

$(\mathrm{e})_{\kappa}^{n^{*}}(\alpha) A_{\zeta} \subseteq \delta$ for $\zeta<\kappa$ are pairwise disjoint,

$(\beta) e$ is an equivalence relation on $\delta$ such that each equivalence class is a finite interval,

$(\gamma)$ for every $n<n^{*}, B \in J$ and $\zeta<\kappa$, for some $\alpha<\delta$ we have $\alpha / e \subseteq A_{\zeta} \backslash B$ and $|\alpha / e| \geq n$.

Then there is a Boolean algebra $B$ of cardinality $\lambda$ as in part (1) but with $(\oplus)_{\lambda}^{B}$ strengthened to

$(\circledast)_{\lambda, n^{*}}^{B} \quad$ if :

( $\alpha) a_{\alpha} \in B$ for $\alpha<\lambda$ are distinct,

( $\beta) n<n^{*}$,

$(\gamma) B_{n}^{*}$ is the finite Boolean algebra of subsets of $n \times(n+1)$, and for $l \leq n, f_{l}: n \rightarrow n$ is a function such that

$$
l<m<n \& i<n \Rightarrow f_{l}(i) \neq f_{m}(i)
$$


and

$$
x_{l}=\left\{(i, j): i<n, j<n+1, j \leq f_{l}(i)\right\}
$$

then for some $\alpha_{0}<\ldots<\alpha_{n-1}<\lambda$, the quantifier free type which $\left\langle a_{\alpha_{0}}, \ldots, a_{\alpha_{n-1}}\right\rangle$ realizes in $B$ is equal to the quantifier free type which $\left\langle x_{0}, \ldots, x_{n-1}\right\rangle$ realizes in $B^{*}$.

Rem ark. (1) The case $\delta=\sup _{i<\delta} \lambda_{i}$ is included.

(2) Of course, no order Boolean algebra of cardinality $\lambda$ can satisfy $(\oplus)_{\lambda}^{B}$.

(3) Again, $B$ has density $\mu$ and if $\left\langle f_{\alpha}: \alpha<\lambda\right\rangle$ is $\mu$-free then $B$ has exact density $\mu$.

Proof of Lemma 4.2. We shall prove only part (1) as the proof of (2) is similar. Without loss of generality $\delta$ is additively indecomposable.

We define $B$ as an algebra of subsets of $Y=\bigcup_{i<\delta} Y_{2 i}$ where $Y_{i}=\left\{f_{\alpha}\lceil i\right.$ : $\alpha<\lambda\}$ for $i<\delta$. For each $i<\delta$ we can find subsets $F_{2 i, \zeta}($ for $\zeta<\kappa$ ) of $Y_{2 i}$ such that for any disjoint subsets $X_{1}, X_{2}$ of $Y_{2 i}$, each of cardinality $<\sigma$, for some $\zeta<\kappa$ we have $F_{2 i, \zeta} \cap\left(X_{1} \cup X_{2}\right)=X_{1}$ (possible as $\kappa=\kappa^{<\sigma}$ and $2^{\kappa} \geq \lambda_{2 i+1}>\left|Y_{2 i}\right|$, by [EK] or see [Sh g, Appendix 1.10]). We can find a sequence $\left\langle\left(j_{i}, \zeta_{i}\right): i<\delta\right\rangle$ such that $j_{i} \leq i<\delta, \zeta_{i}<\kappa$ and for an unbounded set of $j<\delta$ for every $\zeta<\kappa$ for some $\varepsilon<\kappa$ we have

$$
\left\{2 i, 2 i+1: 2 i<\delta \& 2 i, 2 i+1 \in A_{\varepsilon}\right\} \subseteq\left\{2 i, 2 i+1<\delta:\left(j_{i}, \zeta_{i}\right)=(j, \zeta)\right\}
$$

(we use $\operatorname{cf}|\delta|=\operatorname{cf}(\mu) \leq \kappa$ ). Now for each $\alpha<\lambda$ we define a set $Z_{\alpha} \subseteq Y$ by $f \in Z_{\alpha}$ if and only if for some $i<\delta$ :

$(\alpha) f \uparrow(2 i)=f_{\alpha} \uparrow(2 i)$

( $\beta) f \uparrow(2 i+2) \neq f_{\alpha} \uparrow(2 i+2)$,

$(\gamma) f \uparrow\left(2 j_{i}\right) \in F_{2 j_{i}, \zeta_{i}} \Rightarrow f(2 i) \leq f_{\alpha}(2 i) \& f(2 i+1) \leq f_{\alpha}(2 i+1)$,

( $\delta) f \uparrow\left(2 j_{i}\right) \notin F_{2 j_{i}, \zeta_{i}} \Rightarrow f(2 i) \geq f_{\alpha}(2 i) \& f(2 i+1) \geq f_{\alpha}(2 i+1)$.

Let $B$ be the $\sigma$-complete Boolean algebra of subsets of $Y$ generated by the family $\left\{Z_{\alpha}: \alpha<\lambda\right\}$. For $f \in Y$ let $[f]=\{g \in Y: g$ extends $f\}$. For notational simplicity let $\sigma=\aleph_{0}$.

Claim 4.2.1. If for $l=1,2, Z^{l} \in B$ are the same Boolean combinations of $Z_{\alpha_{0}^{l}}, \ldots, Z_{\alpha_{n-1}^{l}}$, say $Z^{l}=\tau\left[Z_{\alpha_{0}^{l}}, \ldots, Z_{\alpha_{n-1}^{l}}\right]$ (where $\tau$ is a Boolean term) and $i<\delta$ is such that $\left\langle f_{\alpha_{m}^{l}} \uparrow(2 i): m<n\right\rangle$ is with no repetition and $(\forall m<n)$ $\left(f_{\alpha_{m}^{1}} \uparrow(2 i)=f_{\alpha_{m}^{2}} \uparrow(2 i)\right)$ then:

(a) $Z^{1} \backslash \bigcup_{m<n}\left[f_{\alpha_{m}^{1}} \uparrow(2 i)\right]=Z^{2} \backslash \bigcup_{m<n}\left[f_{\alpha_{m}^{2}} \uparrow(2 i)\right]$,

(b) for each $m<n$ either

$(\alpha) Z^{1} \cap\left[f_{\alpha_{m}^{1}}\lceil(2 i)]=\left[f_{\alpha_{m}^{1}}\lceil(2 i)] \cap Z_{\alpha_{m}^{1}}\right.\right.$ and $Z^{2} \cap\left[f_{\alpha_{m}^{2}} \uparrow(2 i)\right]=\left[f_{\alpha_{m}^{2}} \uparrow(2 i)\right] \cap Z_{\alpha_{m}^{2}}$, or

(及) $Z^{1} \cap\left[f_{\alpha_{m}^{1}} \uparrow(2 i)\right]=\left[f_{\alpha_{m}^{1}} \uparrow(2 i)\right] \backslash Z_{\alpha_{m}^{1}}$ and $Z^{2} \cap\left[f_{\alpha_{m}^{2}} \uparrow(2 i)\right]=\left[f_{\alpha_{m}^{2}} \uparrow(2 i)\right] \backslash Z_{\alpha_{m}^{2}}$. 
Proof. Check the definition of $Z_{\alpha} \cdot \mathbf{m}_{4.2 .1}$

Clearly $B$ is a Boolean algebra of cardinality $\lambda$. Now the proof of " $B$ has no chain of cardinality $\lambda$ " is similar to the proof of $3.1,3.2$ on noting that for each $i$,

$(*) \quad$ if

(๑) $\Gamma \subseteq \lambda_{2 i} \times \lambda_{2 i+1}$ and for arbitrarily large $\alpha<\lambda_{2 i}$, for arbitrarily large $\beta<\lambda_{2 i+1}$ we have $(\alpha, \beta) \in \Gamma$

then we can find $\left(\alpha_{1}, \beta_{1}\right),\left(\alpha_{2}, \beta_{2}\right) \in \Gamma$ such that $\alpha_{1}<\alpha_{2} \& \beta_{1}<\beta_{2}$.

Up to now, the use of the pairs $2 i, 2 i+1$ was not necessary. But in the proof of " $B$ has no pie of cardinality $\lambda$ ", instead of $(*)$ we use:

$(* *) \quad$ if $(\odot)$ of $(*)$ holds then we can find $\left(\alpha_{1}, \beta_{1}\right),\left(\alpha_{2}, \beta_{2}\right) \in \Gamma$ such that $\alpha_{1}<\alpha_{2} \& \beta_{1}>\beta_{2}$,

easily finishing the proof. $\mathbf{m}_{4.2}$

Conclusion 4.3. For a class of cardinals $\lambda$, there is a Boolean algebra $B$ of cardinality $\lambda^{+}$with no chain and no pie of cardinality $\lambda^{+}$. [We can say, in fact, that this holds for many $\lambda$.]

Pr o of. For any regular $\kappa$, if $2^{\kappa}>\aleph_{\kappa^{+4}}$ then (by [Sh 400, §4]) for some club $E$ of $\kappa^{+4}$,

$$
\mathfrak{a} \subseteq\left(\kappa^{+5}, \aleph_{\kappa^{+4}}\right) \&|\mathfrak{a}| \leq \kappa \& \sup (\mathfrak{a})<\aleph_{\delta} \& \delta \in E \Rightarrow \operatorname{maxpcf}(\mathfrak{a})<\aleph_{\delta} .
$$

Next we choose by induction on $i<\kappa$ cardinals $\lambda_{i} \in \operatorname{Reg} \cap\left[\kappa^{+4}, \aleph_{\kappa^{+4}}\right]$, $\lambda_{i}>\max \operatorname{pcf}\left(\left\{\lambda_{j}: j<i\right\}\right)$. Let $\mu$ be minimal such that $\mu \geq \sup _{i<\kappa} \lambda_{i}$ and $\mu \in \operatorname{pcf}\left(\left\{\lambda_{i}: i \in \kappa\right\}\right)$. Then (by [Sh 345a, 1.8], replacing $\left\{\lambda_{i}: i<\kappa\right\}$ by a subset of the same cardinality, noting $\left\{\lambda_{i}: i \in A\right\} \in J_{<\mu}\left[\left\{\lambda_{i}: i<\kappa\right\}\right]$ when $\left.A \in[\kappa]^{<\kappa}\right)$ we have $\mu=\operatorname{tcf}\left(\prod_{i<\kappa} \lambda_{i} / J_{\kappa}^{\text {bd }}\right)$. Also, as $\mu \in\left[\kappa, \aleph_{\kappa^{+4}}\right]$ is regular, it is a successor cardinal; now the conclusion follows by 4.2 .

Generally, for any $\kappa$ let $\alpha_{0}=0, \lambda^{0}=\kappa^{+}$. By induction on $n$, choose $\alpha_{n+1},\left\langle\lambda_{i}: \alpha_{n} \leq i<\alpha_{n+1}\right\rangle, u_{n}$ and regular $\lambda^{n+1}$ such that $\alpha_{n+1}=\alpha_{n}+\lambda^{n}$ and $\left\langle\lambda_{i}: \alpha_{n} \leq i<\alpha_{n+1}\right\rangle$ is a strictly increasing sequence of regular cardinals in $\left[\lambda^{n}, \aleph_{\left(\lambda^{n}\right)^{+4}}\right]$ such that $\lambda_{i}>\max \operatorname{pcf}\left(\left\{\lambda_{j}: \alpha_{n} \leq j<i\right\}\right.$ ) (possible by [Sh 400, §2] as above). Let $u_{n} \subseteq\left[\alpha_{n}, \alpha_{n+1}\right)$ with $\left|u_{n}\right|=\lambda^{n}$ be such that $\prod_{i \in u_{n}} \lambda_{i} / J_{u_{n}}^{\text {bd }}$ has a true cofinality which we call $\lambda^{n+1}$. Lastly, for some infinite $v \subseteq \omega, \prod_{n \in v} \lambda_{n} / J_{v}^{\text {bd }}$ has a true cofinality, which we call $\lambda$. By renaming $v=\omega, u_{n}=\left[\alpha_{n}, \alpha_{n+1}\right)$. Then $\delta:=\sup _{n} \alpha_{n}, \lambda$ and $\left\langle\lambda_{i}: i<\delta\right\rangle$ are as required in 4.2 if we let

$$
J=\left\{u \subseteq \delta: \text { for every large enough } n, \sup \left(u \cap \alpha_{n}\right)<\alpha_{n}\right\} .
$$


One point is left: why is $\lambda$ a successor cardinal? For it is in $\left[\sup _{n<\omega} \lambda_{n}\right.$, $\left.\prod_{n<\omega} \lambda_{n}\right]$ and either

$$
\prod_{n<\omega} \lambda_{n} \leq\left[\sup _{n<\omega} \lambda_{n}\right]^{\aleph_{0}} \leq 2^{\sum_{n} \lambda_{n}}<\aleph_{\left(\sum_{n<\omega} \lambda_{n}\right)^{+4}}
$$

or the first attempt succeeds for $\kappa=\sum_{n<\omega} \lambda^{n} \cdot \mathbf{m}_{4.3}$

We have actually proved the existence of many such objects. If we waive some requirements, even more.

Proposition 4.4. For any regular cardinal $\theta$ we can find $\delta, J, \lambda, \lambda_{i}$ $($ for $i<\delta)$ as in 4.2 and such that:

(x) $\lambda$ is a successor cardinal,

(y) for each $i$, for some regular cardinal $\mu_{i}$ we have $\lambda_{i}=\mu_{i}^{+}$and $\left(\mu_{i}\right)^{\theta}$ $=\mu_{i}$

(z) one of the following occurs:

(i) $\delta$ is a regular cardinal $\left\langle\lambda_{0}, \delta>0\right.$ and $J=J_{\delta}^{\mathrm{bd}}$,

(ii) $\delta=\beth_{\delta}$ has cofinality $\theta$, and for some $\lambda^{j}(j<\operatorname{cf}(\delta))$ we have

$$
\begin{aligned}
J & =\left\{a: a \subseteq \delta,\left\{j<\operatorname{cf}(\delta): a \cap \lambda^{j} \notin J_{\lambda^{j}}^{\mathrm{bd}}\right\} \in J_{\mathrm{cf}(\delta)}^{\mathrm{bd}}\right\} \\
\text { and } \mu_{\alpha} & =\left(\mu_{\alpha}\right)^{\sup \left\{\lambda_{i}: i<\alpha\right\}} \text {. }
\end{aligned}
$$

Lemma 4.5. Assume $\left\langle\lambda_{i}: i<\delta\right\rangle, \lambda, J, \sigma,\left\langle f_{\alpha}: \alpha<\lambda\right\rangle$ are as in (a)-(d) of 4.2(1) and:

$\left(\mathrm{e}^{\prime}\right)$ for every $B \in J$ for some $i<\delta$ we have $2 i \notin B \& 2 i+1 \notin B$,

$\left(\mathrm{f}^{\prime}\right)$ for every $i<\delta$ we have $\operatorname{Ens}_{\sigma}\left(\lambda_{i}, \lambda_{i}\right)$ or at least for some club $C$ of $\delta$, if $i<\delta$ and $i=\sup \{j \in C:|i \cap C \backslash j| \geq 1\}$ then $\operatorname{Ens}_{\sigma}\left(\lambda_{2 i}\right.$, $\left.\left|\left\{f_{\alpha} \uparrow(2 i): \alpha<\lambda\right\}\right|\right)$.

Then the conclusion of 4.2(1) holds. [We can weaken $\left(\mathrm{f}^{\prime}\right)$ as in 3.3(6).]

Proof. For each $i<\delta$ let $\left\langle\left(\lambda_{2 i},<_{\eta}^{2 i}\right): \eta=f_{\alpha} \uparrow(2 i)\right.$ for some $\left.\alpha<\lambda\right\rangle$ be a $\sigma$-entangled sequence of linear orders (each of cardinality $\lambda_{2 i}$ ).

Now repeat the proof of 4.2 with no $F_{2 i}$ 's, but defining $Z_{\alpha}$ we let $f \in Z_{\alpha}$ if and only if for some $i<\delta$, letting $j=2 i$ or be as in clause $\left(\mathrm{f}^{\prime}\right)$ for $2 i$ we have

$$
\begin{array}{cl}
f \uparrow(2 i)=f_{\alpha} \uparrow(2 i), & f \uparrow(2 i+2) \neq f_{\alpha} \uparrow(2 i+2), \\
f(2 i) \leq_{f \uparrow(2 i)}^{2 i} f_{\alpha}(2 i) \text { and } & f(2 i+1) \leq_{f \uparrow(2 i)}^{2 i} f_{\alpha}(2 i+1) . \mathbf{\square}_{4.5}
\end{array}
$$

Discussion. Now instead of using on each set $\left\{\eta \frown\langle\alpha\rangle: \alpha<\lambda_{\lg (\eta)}\right\}$ a linear order we can use a partial order; we can combine 4.6 below with $4.2(2)$ or with any of our proofs involving pcf for the existence of an entangled linear order. 
Lemma 4.6. (1) Assume $\left\langle\lambda_{i}: i<\delta\right\rangle, \lambda, J, \sigma,\left\langle f_{\alpha}: \alpha<\lambda\right\rangle$ are as in (a)-(d) of 4.2(1) and

(e) for each $i<\delta$ there is a sequence $\bar{P}=\left\langle P_{\varepsilon}: \varepsilon<\kappa_{i}\right\rangle$ where $\kappa_{i}=\left|\left\{f_{\alpha} \mid i: \alpha<\lambda\right\}\right|$, each $P_{\varepsilon}$ is a partial order and $\bar{P}$ is $(\lambda, \sigma)$-entangled, which means: if $u_{0}, u_{1}, u_{2}$ are disjoint subsets of $\kappa_{i}$ of cardinality $<\sigma$ and for $\varepsilon \in u_{0} \cup u_{1} \cup u_{2}, t_{\alpha}^{\varepsilon} \in P_{\varepsilon}$ (for $\alpha<\lambda$ ) are pairwise distinct then for some $\alpha<\beta$,

$$
\begin{aligned}
& \varepsilon \in u_{0} \Rightarrow P_{\varepsilon} \models t_{\alpha}^{\varepsilon}<t_{\beta}^{\varepsilon}, \\
& \varepsilon \in u_{1} \Rightarrow P_{\varepsilon} \models t_{\alpha}^{\varepsilon}>t_{\beta}^{\varepsilon}, \\
& \varepsilon \in u_{2} \Rightarrow P_{\varepsilon} \models " t_{\alpha}^{\varepsilon}, t_{\beta}^{\varepsilon} \text { are incomparable". }
\end{aligned}
$$

Then the conclusion of $4.2(1)$ holds.

(2) Assume as in (1) but:

$\left(\mathrm{e}^{\prime}\right)$ for some $A \subseteq \delta, A \notin J$ and $\delta \backslash A \notin J$,

$\left(\mathrm{e}^{\prime}\right)_{1}$ like (e) of 4.6(1) for $i \in A$ with $u_{2}=\emptyset$,

$\left(\mathrm{e}^{\prime}\right)_{2}$ like (e) of 4.6(1) for $i \in \delta \backslash A$ with $u_{0}=u_{1}=\emptyset, u_{2}=1$ (so we can use $\left.P_{\alpha}=\left(\lambda_{i},=\right)\right)$.

Then the conclusion of $4.2(1)$ holds.

(3) We can weaken " $\kappa_{i}=\mid\left\{f_{\alpha}\lceil i: \alpha<\lambda\} \mid\right.$ " as in $4.5\left(\mathrm{f}^{\prime}\right)$.

P r o o f. Similar to earlier ones. 4.6

\section{More on entangledness}

Proposition 5.1. Suppose that $\left\langle\lambda_{i}: i<i(*)\right\rangle$ is a strictly increasing sequence of regular cardinals, $T_{i} \subseteq \lambda_{i}>2$ is closed under initial segments, $i+1<i(*) \Rightarrow\left|T_{i}\right|<\lambda_{i+1}$ and the set

$$
B_{i}=\left\{\eta \in{ }^{\lambda_{i}} 2: \text { for every } \alpha<\lambda_{i}, \eta\left\lceil\alpha \in T_{i}\right\}\right.
$$

has cardinality $\geq \mu=\operatorname{cf}(\mu)>\lambda_{i}+\left|T_{i}\right|($ for each $i<i(*))$. Then $\left\langle\left(B_{i},<_{\mathrm{lx}}\right)\right.$ : $i<i(*)\rangle$ is a $\left(\mu, \aleph_{0}\right)$-entangled sequence of linear orders $\left(<_{\mathrm{lx}}\right.$ is the lexicographic order).

Remark 5.2. So if $\mu=\operatorname{cf}(\mu)$ and $\theta=\mid\left\{\lambda: \lambda<\mu \leq 2^{\lambda}\right.$ and $\left.2^{<\lambda}<2^{\lambda}\right\} \mid$ then $\operatorname{Ens}(\mu, \theta)$ (see [Sh 430, 3.4]).

Proof of Proposition 5.1. Clearly $\left|T_{i}\right| \geq \lambda_{i}$ (as $\left.B_{i} \neq \emptyset\right)$. So let $n<\omega, i_{0}<i_{1}<\ldots<i_{n-1}<i(*)$, and $\eta_{\zeta}^{l} \in B_{i_{l}}$ for $l<n, \zeta<\mu$ be such that

$$
\zeta<\xi<\mu \& l<n \Rightarrow \eta_{\zeta}^{l} \neq \eta_{\xi}^{l}
$$

and let $u \subseteq n$. We should find $\zeta<\xi<\mu$ such that $(\forall l<n)\left(\eta_{\zeta}^{l}<_{\mathrm{lx}} \eta_{\xi}^{l} \Leftrightarrow\right.$ $i \in u$ ). To this end we prove by downward induction on $m \leq n$ that (stipulating $\lambda_{n}=\mu$ ) 
$(*)_{m} \quad$ there is a set $w \subseteq \mu$ of cardinality $\geq \lambda_{i_{m}}$ such that if $m \leq l<n$ and $\zeta<\xi$ are from $w$ then $\left[\eta_{\zeta}^{l}<_{\mathrm{lx}_{\mathrm{x}}} \eta_{\xi}^{l}\right]^{\text {if }(l \in u)}$.

Note that $(*)_{n}$ is exemplified by $w:=\mu$ and $(*)_{0}$ says (more than) that the conclusion holds, so this suffices. Hence assume $(*)_{m+1}$ is exemplified by $w^{*}$ and we shall find $w \subseteq w^{*}$ exemplifying $(*)_{m}$ with $|w|=\lambda_{i_{m}}$. Without loss of generality $m \in u$ (otherwise replace each $\eta \in T_{i_{m}} \cup\left\{\eta_{\zeta}^{m}: \zeta \in w^{*}\right\}$ by $\langle 1-\eta(\alpha): \alpha<\lg (\eta)\rangle)$. For $\alpha<\lambda_{i_{m}}$ and $\nu \in T_{i} \cap^{\alpha} 2$ let

$$
\begin{gathered}
w_{\nu}^{*}:=\left\{\zeta \in w^{*}: \nu=\eta_{\zeta}^{m}\left\lceil\alpha, \neg\left(\exists \xi \in w^{*}\right)\left(\xi<\zeta \& \eta_{\xi}^{m}\left\lceil\alpha=\nu \& \eta_{\xi}^{m}<_{\mathrm{lx}} \eta_{\zeta}^{m}\right)\right\},\right.\right. \\
w_{\alpha}^{*}=\bigcup\left\{w_{\nu}^{*}: \nu \in T_{i_{m}} \cap^{\alpha} 2\right\} .
\end{gathered}
$$

As in $\left(B_{i_{m}},<_{\mathrm{lx}}\right)$ there is no monotonic sequence of length $\lambda_{i_{m}}^{+}$, clearly $\left|w_{\nu}^{*}\right|$ $\leq \lambda_{i_{m}}$. Moreover,

$$
\left|w_{\alpha}^{*}\right| \leq\left|\left\{\nu \in T_{i_{m}}: \lg (\nu)=\alpha\right\}\right| \times \sup \left\{\left|w_{\nu}^{*}\right|: \nu \in T_{i_{m}} \cap{ }^{\alpha} 2\right\} \leq\left|T_{i_{m}}\right| \times \lambda_{i_{m}},
$$

and hence $\left|\bigcup_{\alpha<\lambda_{i_{m}}} w_{\alpha}^{*}\right| \leq \lambda_{i_{m}}+\left|T_{i_{m}}\right|$. But $\left|T_{i_{m}}\right|<\lambda_{i_{m+1}}$ and $\lambda_{i_{m}}<\lambda_{i_{m+1}}$. Hence we find $\zeta(*) \in w^{*} \backslash \bigcup_{\alpha<\lambda_{i_{m}}} w_{\alpha}^{*}$. Now, for every $\alpha<\lambda_{i_{m}}$ let $\xi_{\alpha} \in w^{*}$ exemplify $\zeta(*) \notin w_{\eta_{\zeta}^{m}(*)\lceil\alpha}^{*} \subseteq w_{\alpha}^{*}$, so

$$
\xi_{\alpha}<\zeta(*), \quad \eta_{\xi_{\alpha}}^{m}\left\lceil\alpha=\eta_{\zeta(*)}^{m}\left\lceil\alpha \quad \text { and } \quad \eta_{\xi_{\alpha}}^{m}<_{\mathrm{lx}} \eta_{\zeta(*)}^{m} .\right.\right.
$$

Then for some $\gamma_{\alpha}$ with $\alpha \leq \gamma_{\alpha}<\lambda_{i_{m}}$, we have

$$
\eta_{\zeta(*)}^{m}\left\lceil\gamma_{\alpha}=\eta_{\xi_{\alpha}}^{m}\left\lceil\gamma_{\alpha}, \quad \eta_{\zeta(*)}^{m}\left(\gamma_{\alpha}\right)=1, \quad \eta_{\xi_{\alpha}}^{m}\left(\gamma_{\alpha}\right)=0\right.\right.
$$

So for some unbounded set $A \subseteq \lambda_{i}$ the sequence $\left\langle\gamma_{\alpha}: \alpha \in A\right\rangle$ is strictly increasing in $\alpha$ and also $\left\langle\xi_{\alpha}: \alpha \in A\right\rangle$ is increasing. Let $w:=\left\{\xi_{\alpha}: \alpha \in A\right\} \subseteq$ $w^{*}$. It exemplifies $(*)_{m}$, hence we finish. $\mathbf{\square}_{5.1}$

Proposition 5.3. (1) Assume that:

(a) $\lambda=\max \operatorname{pcf}\left(\mathfrak{a}_{\varepsilon}\right)$ for $\varepsilon<\varepsilon(*)$,

(b) $\left|\mathfrak{a}_{\varepsilon}\right| \leq \kappa<\kappa^{*} \leq \min \left(\mathfrak{a}_{\varepsilon}\right)$,

(c) $\theta \in \mathfrak{a}_{\varepsilon} \Rightarrow \theta$ is $\left(\kappa^{*}, \kappa^{+}, 2\right)$-inaccessible,

(d) for $n<\omega$ and distinct $\varepsilon_{0}, \varepsilon_{1}, \ldots, \varepsilon_{n}<\varepsilon(*)$ we have

$$
\mathfrak{a}_{\varepsilon_{0}} \backslash \bigcup_{l=1}^{n} \mathfrak{a}_{\varepsilon_{l}} \notin J_{<\lambda}\left[\mathfrak{a}_{\varepsilon_{0}}\right] .
$$

Then $\operatorname{Ens}(\lambda, \lambda, \varepsilon(*))$.

(2) Assume in addition that:

(d) if $u \in[\varepsilon(*)]^{<\sigma}$ and $\varepsilon \in \varepsilon(*) \backslash u$ then $\mathfrak{a}_{\varepsilon} \backslash \bigcup_{\zeta \in u} \mathfrak{a}_{\zeta} \notin J_{<\lambda}\left[\mathfrak{a}_{\varepsilon}\right]$,

(e) if $\theta \in \mathfrak{a}_{\varepsilon}$ then $\max \operatorname{pcf}\left(\bigcup_{\zeta} \mathfrak{a}_{\zeta} \cap \theta\right)<\theta$,

(f) $(\forall \alpha<\lambda)\left(|\alpha|^{<\sigma}<\lambda\right)$.

Then $\operatorname{Ens}_{\sigma}(\lambda, \lambda, \varepsilon(*))$.

Proof. As in $\S 3$. 
Proposition 5.4. For any cardinal $\lambda$ satisfying $(\forall \kappa<\lambda)\left(2^{\kappa}<2^{\lambda}\right)$ there is a successor cardinal $\theta \in\left[\lambda, 2^{\lambda}\right]$ such that there is an entangled linear order of cardinality $\theta$.

P r o of. We prove slightly more, so let $\lambda$ and $\chi \in\left[\lambda, 2^{\lambda}\right]$ be any cardinals (we shall try to find $\theta^{+} \in\left[\chi, 2^{\lambda}\right]$ for $\chi$ as below; for the proposition $\mu=\lambda$ below).

Let $\mu:=\min \left\{\mu: 2^{\mu}=2^{\lambda}\right\}$, so $\mu \leq \lambda$ and $\mu<\operatorname{cf}\left(2^{\mu}\right)$ and $\kappa<\mu \Rightarrow$ $2^{\kappa}<2^{\mu}$. First assume $2^{<\mu}=2^{\mu}$. Then necessarily $\mu$ is a limit cardinal. If $\operatorname{cf}\left(2^{<\mu}\right)=\operatorname{cf}(\mu)$ we get a contradiction with the previous sentence. Hence $\left\langle 2^{\theta}: \theta<\mu\right\rangle$ is eventually constant so for some $\theta<\mu$ we have $2^{\theta}=2^{<\mu}$; but $2^{<\mu}=2^{\mu}$, a contradiction to the choice of $\mu$. Thus we have $2^{<\mu}<2^{\mu}=2^{\lambda}$. Assume $\chi=\lambda+2^{<\mu}$ or just $2^{\lambda}>\chi \geq 2^{<\mu}$. The proof splits into cases: if $\operatorname{cf}\left(2^{\lambda}\right)$ is a successor, use cases $\mathrm{B}$ or $\mathrm{C}$ or $\mathrm{D}$, if $\mathrm{cf}\left(2^{\lambda}\right)$ is a limit cardinal (necessarily $>\lambda$ ) use case A.

Case A: $\chi^{+\left(\mu^{+4}\right)} \leq 2^{\lambda}$. By 3.6(2).

Case B: $\operatorname{cf}\left(2^{\lambda}\right)$ is a successor, $\mu$ is strong limit (e.g. $\left.\aleph_{0}\right)$. Clearly there is a dense linear order of cardinality $\operatorname{cf}\left(2^{\lambda}\right)$ and density $\mu$, hence there is an entangled linear order in $\operatorname{cf}\left(2^{\lambda}\right)$, which is as required ([BoSh 210]).

Cas e $\mathrm{C}$ : $\operatorname{cf}\left(2^{\lambda}\right)$ is a successor cardinal, $\mu$ is regular uncountable. Look at [Sh 410, 4.3] (with $\mu$ here standing for $\lambda$ there); conditions (i) + (ii) hold. Now on the one hand, if assumption (iii) of [Sh 410, 4.3] fails, we know that there is an entangled linear order of cardinality $\mathrm{cf}\left(2^{\lambda}\right)$ (as in Case B). But on the other hand, if (iii) holds, the conclusion of [Sh 410, 4.3] gives more than we asked for. In both cases there is an entangled linear order in $\operatorname{cf}\left(2^{\lambda}\right)$ (which is a successor).

Case D: $\mu$ is singular, not strong limit. By [Sh 430, 3.4] there are regular cardinals $\theta_{i}$ (for $\left.i<\operatorname{cf}(\mu)\right)$ such that the sequence $\left\langle\theta_{i}: i<\operatorname{cf}(\mu)\right\rangle$ is increasing with limit $\mu, \mu<2^{\theta_{i}},\left\langle 2^{\theta_{i}}: i<\operatorname{cf}(\mu)\right\rangle$ is strictly increasing and for all $\sigma$ such that $\sigma=\operatorname{cf}(\sigma) \leq 2^{\theta_{i}}$ there is a tree $T_{\sigma}^{i}$ with $\left|T_{\sigma}^{i}\right|=\theta_{i}$ or at least $2^{\left|T_{\sigma}^{i}\right|}=2^{\theta_{i}}$ and $T_{\sigma}^{i}$ has $\geq \sigma \theta_{i}$-branches.

If for some $i$ either $2^{\theta_{i}}$ is a successor $\geq \chi$ or $\operatorname{cf}\left(2^{\theta_{i}}\right)$ is a successor $\geq \chi$ we finish as in Case B; if $\operatorname{cf}\left(2^{\theta_{i}}\right)$ is a limit cardinal, we finish as in Case A (or use 5.5).

Proposition 5.5. Assume $2^{\mu}$ is singular. Then there is an entangled linear order of cardinality $\left(2^{\mu}\right)^{+}$.

P r o of. Let $\lambda$ be the first singular cardinal $>\mu$ such that $(\exists \kappa<\lambda)\left(\operatorname{pp}_{\kappa}(\lambda)\right.$ $>2^{\mu}$ ). Now, $\lambda$ is well defined, and moreover $\lambda \leq 2^{\mu}$ (as $2^{\mu}$ is singular so $\operatorname{pp}\left(2^{\mu}\right) \geq\left(2^{\mu}\right)^{+}>2^{\mu}$ ) and (by [Sh 355, 2.3], [Sh 371, 1.9])

$$
\operatorname{cf}(\lambda)<\chi \in(\mu, \lambda) \backslash \operatorname{Reg} \Rightarrow \operatorname{pp}_{\operatorname{cf}(\lambda)+\operatorname{cf}(\chi)}(\chi)<\lambda,
$$


so $\operatorname{pp}(\lambda)>2^{\mu}$ and $\operatorname{cf}(\lambda)>\mu$ (otherwise $\left.\operatorname{pp}(\lambda) \leq \lambda^{\operatorname{cf}(\lambda)} \leq \lambda^{\mu} \leq\left(2^{\mu}\right)^{\mu}=2^{\mu}\right)$. Lastly, apply 3.2(1). $\mathbf{\square}_{5.5}$

Proposition 5.6. If $\kappa^{+}<\chi_{0}<\lambda$ and $\kappa^{+4}<\operatorname{cf}(\lambda)<\lambda \leq 2^{\kappa}$, then either

(a) there is a strictly increasing sequence $\left\langle\lambda_{i}^{*}: i<\delta\right\rangle$ of regular cardinals from $\left(\chi_{0}, \lambda\right)$ with $\delta=\operatorname{cf}(\delta) \in[\kappa, \operatorname{cf}(\lambda)] \cap \operatorname{Reg}$ and $\lambda_{i}^{*}>\max \operatorname{pcf}\left(\left\{\lambda_{j}^{*}: j<i\right\}\right)$ such that $\lambda^{+}=\operatorname{tcf}\left(\prod_{i<\delta} \lambda_{i}^{*} / J_{\delta}^{\mathrm{bd}}\right)$, or

(b) there is a strictly increasing sequence $\left\langle\lambda_{i}^{*}: i<\delta\right\rangle$ of regular cardinals from $(\chi, \lambda)$ such that $\lambda_{i}^{*}>\max \operatorname{pcf}\left(\left\{\lambda_{j}^{*}: j<i\right\}\right)$ and $\operatorname{Ens}\left(\lambda_{i}^{*}, \lambda_{i}^{*}\right)$ and $\lambda^{+}=\operatorname{tcf}\left(\prod_{i<\delta} \lambda_{i}^{*} / I\right)$, with I a proper ideal on $\delta$ extending $J_{\delta}^{\mathrm{bd}}$. Moreover for some $\mu \in\left(\chi_{0}, \lambda\right), \mu<\lambda_{0}^{*}$ and there is a sequence $\left\langle\mathfrak{b}_{i, j}: i<\delta, j<\kappa^{+}\right\rangle$ such that $\mathfrak{b}_{i, j} \subseteq \operatorname{Reg} \cap \mu \backslash \chi_{0},\left|\mathfrak{b}_{i, j}\right| \leq \kappa$, each $\theta \in \bigcup_{i, j} \mathfrak{b}_{i, j}$ is $\left(\chi_{0}, \kappa^{+}, \aleph_{0}\right)$ inaccessible (i.e. $\mathfrak{a} \subseteq \operatorname{Reg} \cap \theta \backslash \chi_{0},|\mathfrak{a}| \leq \kappa \Rightarrow \max \operatorname{pcf}(\mathfrak{a})<\theta$ ) and

$$
\begin{gathered}
j_{1}<j_{2}<\kappa^{+} \Rightarrow \mathfrak{b}_{i, j_{1}} \cap \mathfrak{b}_{i, j_{2}}=\emptyset, \quad \lambda_{i}^{*}=\max \operatorname{pcf}\left(\mathfrak{b}_{i, j}\right), \\
\mu=\sup \left(\mathfrak{b}_{i, j}\right), \quad J_{\mathfrak{b}_{i, j}}^{\text {bd }} \subseteq J_{<\lambda_{i}^{*}}\left[\mathfrak{b}_{i, j}\right] .
\end{gathered}
$$

(This implies $\operatorname{Ens}\left(\lambda_{i}^{*}, \lambda_{i}^{*}\right)$.)

Remark 5.7. (1) Why $\lambda^{+}$instead of $\lambda^{*}=\operatorname{cf}\left(\lambda^{*}\right) \in\left(\lambda, \operatorname{pp}_{J_{\mathrm{cf}(\lambda)}^{\text {bd }}}^{+}(\lambda)\right)$ ? To be able to apply [Sh 410,3.3] in case III of the proof of [Sh 410, 4.1]. So if $\left\langle\lambda_{i}: i<\operatorname{cf}(\lambda)\right\rangle$ fits in such a theorem we can get $\lambda^{+}$.

(2) We could have improved the theorem if we knew that always

$$
\operatorname{cf}\left([\lambda]^{\leq \kappa}, \subseteq\right)=\lambda+\sup \left\{\operatorname{pp}_{\kappa}(\mu): \operatorname{cf}(\mu) \leq \kappa<\mu<\lambda\right\},
$$

particularly $\sigma$-entangledness.

Proof of Proposition 5.6. This is like the proof of [Sh 410, 4.1]. (In Case II when $\sigma=\aleph_{0}$ imitate [Sh 410, 4.1].) However, after many doubts, for the reader's convenience we present the proof fully, adapting for our purposes the proof of [Sh 410, 4.1].

By [Sh 355, 2.1] there is an increasing continuous sequence $\left\langle\lambda_{i}: i<\right.$ $\operatorname{cf}(\lambda)\rangle$ of singular cardinals with limit $\lambda$ such that $\operatorname{tcf}\left(\prod_{i<\operatorname{cf}(\lambda)} \lambda_{i}^{+},<_{J_{\operatorname{cf}(\lambda)}^{\mathrm{bd}}}\right.$ )$=\lambda^{+}$and $\lambda_{0}>\chi_{0}$. The proof will split into cases. Without loss of generality $\chi_{0}>\operatorname{cf}(\lambda)$.

Case I: $\max \operatorname{pcf}\left(\left\{\lambda_{j}^{+}: j<i\right\}\right)<\lambda$ for $i<\operatorname{cf}(\lambda)$. So for some unbounded $A \subseteq \operatorname{cf}(\lambda)$ we have

$$
(\forall i \in A)\left(\max \operatorname{pcf}\left(\left\{\lambda_{j}^{+}: j \in A \cap i\right\}\right)<\lambda_{i}^{+}\right) .
$$

Consequently, $\mathfrak{a}=\left\{\lambda_{i}^{+}: i \in A\right\}$ satisfies the demands of 3.6 and hence (a) holds true with $\delta=\operatorname{cf}(\lambda), \lambda_{i}^{*}=\lambda_{i}$.

Thus assume that Case I fails. So there is $\mu$ such that $\chi_{0}<\mu<\lambda$, $\operatorname{cf}(\mu)<\operatorname{cf}(\lambda)$ and $\operatorname{pp}_{<\operatorname{cf}(\lambda)}(\mu)>\lambda$. Choose a minimal such $\mu$. Then, by 
[Sh 410, 3.2], we have:

$$
\left[\mathfrak{a} \subseteq \operatorname{Reg} \backslash \chi_{0} \& \sup \mathfrak{a}<\mu \&|\mathfrak{a}|<\operatorname{cf}(\lambda)\right] \Rightarrow \max \operatorname{pcf}(\mathfrak{a})<\lambda .
$$

By $[$ Sh $355,2.3]$ in the conclusion of $(*)$ we may replace " $<\lambda$ " by " $<\mu$ " and we get

$$
(*)^{\prime} \quad\left[\mathfrak{a} \subseteq \operatorname{Reg} \backslash \chi_{0} \& \sup \mathfrak{a}<\mu \&|\mathfrak{a}|<\operatorname{cf}(\lambda)\right] \Rightarrow \max \operatorname{pcf}(\mathfrak{a})<\mu .
$$

Let $\sigma=\operatorname{cf}(\mu)$. Then $\operatorname{pp}(\mu)=\operatorname{pp}_{<\operatorname{cf}(\lambda)}(\mu)$ (and $\operatorname{pp}_{<\operatorname{cf}(\lambda)}(\mu)>\lambda$ ). Without loss of generality $\mu<\lambda_{0}$.

Case II: $\sigma>\kappa$ (and not Case I). By [Sh 371, 1.7], if $\sigma>\aleph_{0}$ and by [Sh 430,6.x] if $\sigma=\aleph_{0}$ we find a strictly increasing sequence $\left\langle\mu_{i}^{*}: i<\sigma\right\rangle$ of regular cardinals, $\mu=\bigcup_{i<\sigma} \mu_{i}^{*}$ and an ideal $J$ on $\sigma$ extending $J_{\sigma}^{\text {bd }}$ (if $\sigma>\aleph_{0}$ then $J=J_{\sigma}^{\mathrm{bd}}$ ) such that

$$
\lambda^{+}=\max \operatorname{pcf}\left(\left\{\mu_{i}^{*}: i<\sigma\right\}\right)=\operatorname{tcf}\left(\prod_{i<\sigma} \mu_{i}^{*} / J\right) .
$$

If $\sigma>\aleph_{0}$, since we may replace $\left\langle\mu_{i}^{*}: i<\sigma\right\rangle$ by $\left\langle\mu_{i}^{*}: i \in A\right\rangle$ for any unbounded $A \subseteq \sigma$, we may assume that $\mu_{i}^{*}>\max \operatorname{pcf}\left(\left\{\mu_{j}^{*}: j<i\right\}\right)$. If $\sigma=\aleph_{0}$ this holds automatically, so in both cases we can apply 3.6. So we get (a), as $\sigma>\kappa \geq \aleph_{0}$.

Cas e III: $\sigma \leq \kappa$. So $\sigma^{+4} \leq \operatorname{cf}(\lambda)$. Let

$\mathcal{P}:=\left\{C \subseteq \operatorname{cf}(\lambda): \operatorname{otp}(C)=\kappa^{+3}, C\right.$ is closed in $\sup (C)$ and

$$
\left.\max \operatorname{pcf}\left(\left\{\lambda_{i}^{+}: i \in C\right\}\right)<\lambda\right\} \text {. }
$$

[Why there are such $C$ 's? For any $\delta<\operatorname{cf}(\lambda)$ with $\operatorname{cf}(\delta)=\kappa^{+3}$ we have a club $C^{\prime}$ of $\lambda_{\delta}$ such that $\operatorname{tcf}\left(\prod_{\theta \in C^{\prime}} \theta^{+} / J_{\kappa^{+3}}^{\mathrm{bd}}\right)=\lambda_{\delta}^{+}$. Now $\left\{i<\delta: \lambda_{i} \in C^{\prime}\right\}$ will do.]

For each $C \in \mathcal{P}$ try to choose, by induction on $i<\kappa^{+}, \mathfrak{b}_{i}=\mathfrak{b}_{i, C}$ and $\gamma_{i}=\gamma_{i, C}$ such that:

(i) $\mathfrak{b}_{i} \subseteq \operatorname{Reg} \cap \mu \backslash \bigcup_{j<i} \mathfrak{b}_{j} \backslash \chi_{0}$

(ii) $\gamma_{i} \in C \backslash \bigcup_{j<i}\left(\gamma_{j}+1\right)$,

(iii) $\lambda_{\gamma_{i}}^{+} \in \operatorname{pcf}\left(\mathfrak{b}_{i}\right)$,

(iv) $\left|\mathfrak{b}_{i}\right| \leq \sigma$,

(v) all members of $\mathfrak{b}_{i}$ are $\left(\chi_{0}, \kappa^{+}, \aleph_{0}\right)$-inaccessible,

(vi) $\gamma_{i}$ is minimal under the other requirements.

Let $\left(\mathfrak{b}_{i, C}, \gamma_{i, C}\right)$ be defined if and only if $i<i_{C}(*)$. So success in defining means $i_{C}(*)=\kappa^{+}$, failure means $i_{C}(*)<\kappa^{+}$.

Subcas e IIIA: For some $j<\operatorname{cf}(\lambda)$, for every $C \in \mathcal{P}$ with $\min (C) \geq j$ we have $i_{C}(*)<\kappa^{+}$, so we cannot define $\mathfrak{b}_{i_{C}(*), C}, \gamma_{i_{C}(*), C}$. Let $C, i_{C}(*)$ be as above. Let $\gamma_{C}^{*}=\bigcup_{i<i_{C}(*)} \gamma_{i, C}$, so $\gamma_{C}^{*} \in C$. Now, if $\gamma \in C \backslash \gamma_{C}^{*}$ then (by $[\operatorname{Sh} 355,1.5 \mathrm{~B}]$ ) as $\operatorname{pp}_{\sigma}(\mu) \geq \lambda^{+}>\lambda_{\gamma}^{+}$, there is $\mathfrak{a}_{\gamma} \subseteq \operatorname{Reg} \cap\left(\chi_{0}, \mu\right)$ with 
$\left|\mathfrak{a}_{\gamma}\right| \leq \sigma$ such that $\lambda_{\gamma}^{+} \in \operatorname{pcf}\left(\mathfrak{a}_{\gamma}\right)$. By $[\operatorname{Sh} 410,3.2]$ there is $\mathfrak{c}_{\gamma} \subseteq \operatorname{Reg} \cap\left(\chi_{0}, \mu\right)$ of cardinality $\leq \kappa$ consisting of $\left(\chi, \kappa^{+}, \aleph_{0}\right)$-inaccessible cardinals such that $\lambda_{\gamma}^{+} \in \operatorname{pcf}\left(\mathfrak{c}_{\gamma}\right)$. Now $\gamma$ and $\mathfrak{c}_{\gamma} \backslash \bigcup_{i<i_{C}(*)} \mathfrak{b}_{i, C}$ cannot serve as $\gamma_{i_{C}(*), C}$ and $\mathfrak{b}_{i_{C}(*), C}$, so necessarily $\lambda_{\gamma}^{+} \notin \operatorname{pcf}\left(\mathfrak{c}_{\gamma} \backslash \bigcup_{i<i_{C}(*)} \mathfrak{b}_{i, C}\right)$. Hence without loss of generality $\mathfrak{c}_{\gamma} \subseteq \bigcup_{i<i_{C}(*)} \mathfrak{b}_{i, C}$. So

$$
\left\{\lambda_{i}^{+}: i \in C \backslash \gamma_{C}^{*}\right\} \subseteq \operatorname{pcf}\left(\bigcup_{i<i_{C}(*)} \mathfrak{b}_{i, C}\right) \quad \text { and }\left|\bigcup_{i<i_{C}(*)} \mathfrak{b}_{i, C}\right| \leq \kappa .
$$

By the proof of $[$ Sh 400, 4.2] (or see [Sh 410, §3]) we get a contradiction (note that $\operatorname{cf}(\lambda)>\kappa^{+4}$ does not disturb).

$\mathrm{S}$ u b c a s e IIIB: For every $j<\operatorname{cf}(\lambda)$ there is $C \in \mathcal{P}$ such that $\min (C)>j$ and $i_{C}(*)=\kappa^{+}$, i.e. $\mathfrak{b}_{i, C}$ and $\gamma_{i, C}$ are defined for every $i<\kappa^{+}$. We will show

$(\otimes) \quad$ for every $j(*)<\operatorname{cf}(\lambda)$ there is $\lambda^{\prime} \in \lambda \cap \operatorname{pcf}\left(\left\{\lambda_{j}^{+}: j<\operatorname{cf}(\lambda)\right\}\right) \backslash \lambda_{j(*)}$ such that:

$(\alpha) \operatorname{Ens}\left(\lambda^{\prime}, \lambda^{\prime}\right)$ (exemplified by a linear order which has density character $>\chi_{0}$ in every interval),

$(\beta)$ for some $\mathfrak{b} \subseteq \mu \cap \operatorname{Reg} \backslash \chi_{0}$ we have $|\mathfrak{b}| \leq \kappa^{+}$and $\lambda^{\prime}=\max \operatorname{pcf}(\mathfrak{b})$. Moreover, $\mathfrak{b}$ can be divided to $\kappa^{+}$subsets of cardinality $\leq \kappa$, no one in $J_{<\lambda^{\prime}}[\mathfrak{b}]$ and

$$
(\forall \theta \in \mathfrak{b})(\theta>\max \operatorname{pcf}(\mathfrak{b} \cap \theta))
$$

(even $\theta$ is $\left(\chi_{0}, \kappa^{+}, \aleph_{0}\right)$-inaccessible).

Why does $(\otimes)$ suffice? Suppose that we have proved $(\otimes)$ already. So for $i<\operatorname{cf}(\lambda)$ we can choose $\mu_{i}^{*}$ with $\lambda_{i}<\mu_{i}^{*}=\operatorname{cf}\left(\mu_{i}^{*}\right) \in \lambda \cap \operatorname{pcf}\left(\left\{\lambda_{j}^{+}: j<\operatorname{cf}(\lambda)\right\}\right)$ as required in $(\otimes)$. Since $(\forall i)\left(\mu_{i}^{*}<\lambda\right)$, we can assume that the sequence $\left\langle\mu_{i}^{*}: i<\operatorname{cf}(\lambda)\right\rangle$ is strictly increasing. By induction on $\varepsilon<\operatorname{cf}(\lambda)$ choose strictly increasing $i(\varepsilon)<\operatorname{cf}(\lambda)$ such that $\mu_{i(\varepsilon)}^{*}>\max \operatorname{pcf}\left(\left\{\mu_{i(\zeta)}^{*}: \zeta<\varepsilon\right\}\right)$.

Let $i(\varepsilon)$ be defined if and only if $\varepsilon<\varepsilon(*)$. So $\varepsilon(*)$ is limit,

$$
\lambda^{+}=\max \operatorname{pcf}\left(\left\{\mu_{i(\varepsilon)}^{*}: \varepsilon<\varepsilon(*)\right\}\right), \quad \mu_{i(\varepsilon)}^{*}>\max \operatorname{pcf}\left(\left\{\mu_{i(\zeta)}^{*}: \zeta<\varepsilon\right\}\right),
$$

$\mu_{i(\varepsilon)}^{*}$ is strictly increasing and $\operatorname{Ens}\left(\mu_{i(\varepsilon)}^{*}, \mu_{i(\varepsilon)}^{*}\right)$. Thus applying [Sh 355, 4.12] we finish, getting clause (b) of 5.6.

Why does $(\otimes)$ hold? Choose $C \subseteq(j(*), \operatorname{cf}(\lambda))$ of order type $\kappa^{+3}$ such that $\left\langle\gamma_{i}=\gamma_{i, C}: i<\kappa^{+}\right\rangle$and $\left\langle\mathfrak{b}_{i}=\mathfrak{b}_{i, C}: i<\kappa^{+}\right\rangle$are well defined and

$$
\max \operatorname{pcf}\left(\left\{\lambda_{\gamma_{i}}^{+}: i \in C\right\}\right)<\lambda
$$

(possible by our being in Subcase IIIB, see the definition of $\mathcal{P}$ ). Let $\mathfrak{d}:=$ $\left\{\lambda_{\gamma_{i}}^{+}: i<\kappa^{+}\right\}$and let $\left\langle\mathfrak{b}_{\theta}[\mathfrak{d}]: \theta \in \operatorname{pcf}(\mathfrak{d})\right\rangle$ be as in [Sh 371, 2.6]. Let $\theta$ be minimal such that $\operatorname{otp}\left(\mathfrak{b}_{\theta}[\mathfrak{d}]\right)=\kappa$. We can find pairwise disjoint sets $B_{\varepsilon} \subseteq C$ (for $\varepsilon<\kappa$ ) such that

$$
\left\{\lambda_{\gamma}^{+}: \gamma \in B_{\varepsilon}\right\} \subseteq \mathfrak{b}_{\theta}[\mathfrak{d}], \quad \operatorname{otp}\left(B_{\varepsilon}\right)=\kappa .
$$


Clearly max $\operatorname{pcf}\left(\left\{\lambda_{\gamma_{i}}^{+}: \gamma_{i} \in B_{\varepsilon}\right\}\right)=\theta$, since $\left\{\lambda_{\gamma_{i}}^{+}: \gamma_{i} \in B_{\varepsilon}\right\} \subseteq \mathfrak{b}_{\theta}[\mathfrak{d}]$ but it is not a subset of any finite union of $\mathfrak{b}_{\theta^{\prime}}[\mathfrak{c}], \theta^{\prime}<\theta$. Now letting $\mathfrak{a}^{*}:=\bigcup_{j \in C} \mathfrak{b}_{j}$, we find (by [Sh 371, 2.6]) a subset $\mathfrak{a}$ of $\mathfrak{a}^{*}$ such that $\theta=\max \operatorname{pcf}(\mathfrak{a})$ but $\theta \notin \operatorname{pcf}\left(\mathfrak{a}^{*} \backslash \mathfrak{a}\right)$. Now as $\theta \in \operatorname{pcf}\left(\left\{\lambda_{\gamma}^{+}: \gamma \in B_{\varepsilon}\right\}\right)$ and $\lambda_{\gamma}^{+} \in \operatorname{pcf}\left(\mathfrak{b}_{\gamma}\right)$ we have (by [Sh 345a, 1.12]) $\theta \in \operatorname{pcf}\left(\bigcup_{\gamma \in B_{\varepsilon}} \mathfrak{b}_{\gamma}\right)$. Hence by the previous sentence $\theta \in \operatorname{pcf}\left(\mathfrak{a} \cap \bigcup_{\gamma \in B_{\varepsilon}} \mathfrak{b}_{\gamma}\right)$. Let

$$
\mathfrak{c}_{\varepsilon}:=\mathfrak{a} \cap \bigcup_{j \in B_{\varepsilon}} \mathfrak{b}_{j}, \quad \lambda^{\prime}=\theta .
$$

We can apply 3.2 to deduce that there is an entangled linear order of cardinality $\lambda^{\prime}$ (which is more than required) and, of course,

$$
\lambda_{j(*)}<\lambda^{\prime} \in \lambda \cap \operatorname{pcf}\left(\left\{\lambda_{j}: j<\operatorname{cf}(\lambda)\right\}\right) .
$$

The assumptions of 3.2 hold as the $\mathfrak{c}_{\varepsilon}$ are pairwise disjoint (by (i) above),

$$
\begin{gathered}
\theta \in \operatorname{pcf}\left(\left\{\lambda_{\gamma_{i}}^{+}: \gamma_{i} \in B_{\varepsilon}\right\}\right), \quad \operatorname{pcf}\left(\bigcup_{j \in B_{\varepsilon}} \mathfrak{b}_{j}\right)=\operatorname{pcf}\left(\mathfrak{c}_{\varepsilon}\right), \\
\theta_{1} \in \mathfrak{a} \Rightarrow \max \operatorname{pcf}\left(\mathfrak{a} \cap \theta_{1}\right)<\theta_{1},
\end{gathered}
$$

as $\theta_{1}$ is $\left(\chi_{0}, \kappa^{+}, \aleph_{0}\right)$-inaccessible and

$$
\theta=\lambda^{\prime} \geq \sup \left\{\lambda_{\gamma_{i}}^{+}: i \in C\right\}>\lambda_{j(*)}>\chi_{0} .
$$

So clause $(\alpha)$ of $(\otimes)$ holds and clause $(\beta)$ was done along the way. Thus we finish Subcase IIIb and hence Case III.

Conclusion 5.8. For $\lambda$ as in 5.6 there is a Boolean algebra $B$ of cardinality $\lambda^{+}$satisfying $(\oplus)_{\lambda^{+}}^{B},(\otimes)_{\sup _{i<\delta} \lambda_{i}^{*}}^{B}$ from 4.1 (and also there is an entangled linear order in $\left.\lambda^{+}\right)$.

P r o of. If (a) of 5.6 holds, apply 4.2. If (b) of 5.6 holds use 4.5. 5.8

Definition 5.9. (1) $\operatorname{pcf}_{\kappa}^{\mathrm{ex}}(\mathfrak{a})=\{\lambda:$ if $\mathfrak{b} \subseteq \mathfrak{a}$ and $|\mathfrak{b}|<\kappa$ then $\lambda \in$ $\operatorname{pcf}(\mathfrak{a} \backslash \mathfrak{b})\}$ (equivalently: $\lambda \in \operatorname{pcf}(\mathfrak{a})$ and $\mathfrak{b} \in J_{<\lambda}[\mathfrak{a}] \Rightarrow\left|\mathfrak{b}_{\lambda}[\mathfrak{a}] \backslash \mathfrak{b}\right| \geq \kappa$ ).

(2) $J_{<\theta}^{\text {ex, }}[\mathfrak{a}]:=\{\mathfrak{b}: \mathfrak{b} \subseteq \mathfrak{a}$ and for some $\mathfrak{c} \subseteq \mathfrak{a}$ we have $|\mathfrak{c}|<\kappa$ and $\left.\mathfrak{b} \backslash \mathfrak{c} \in J_{<\theta}[\mathfrak{a}]\right\}$.

Proposition 5.10. Assume that $\mu$ is a singular cardinal which is a fix point (i.e. $\mu=\aleph_{\mu}$ ) and $\mu^{*}<\mu$.

(1) For some successor cardinal $\lambda^{+} \in\left(\mu, \mathrm{pp}^{+}(\mu)\right)$ there is an entangled linear $\mathcal{I}$ order of cardinality $\lambda^{+}$and density $\in\left(\mu^{*}, \mu\right]$.

(2) If $\mu \leq \chi_{0}$ and $\chi_{0}^{+\mu^{+4}} \leq \mathrm{pp}^{+}(\mu)$ then we can find an entangled linear order $\mathcal{I}$ with $|\mathcal{I}|=\lambda^{+} \in\left(\chi_{0}, \chi_{0}^{+\mu^{+4}}\right)$ of density $\in\left(\mu^{*}, \mu\right]$.

(3) In both (1) and (2), we also get a Boolean algebra $B$ satisfying $(\oplus)_{\lambda^{+}}^{B}$, $(\otimes)_{\mu}^{B}$ of 4.2 . 
(4) In both (1) and (2), if $\mathcal{J}$ is an interval of $\mathcal{I}$ or $\mathcal{J} \in[\mathcal{I}]^{\lambda^{+}}$then $\operatorname{dens}(\mathcal{J})=\operatorname{dens}(\mathcal{I})$. This also applies to 5.10.1-5.10.3.

Proof. Let $\left\langle\mu_{i}: 1 \leq i<\operatorname{cf}(\mu)\right\rangle$ be a strictly increasing continuous sequence with limit $\mu$. We can assume that $\mu_{1}>\mu_{0}>\mu^{*}+\operatorname{cf}(\mu)$ and $\aleph_{0} \leq \operatorname{cf}\left(\mu_{i}\right)<\max \left\{\operatorname{cf}(\mu), \aleph_{1}\right\}$.

(1) We try to choose by induction on $i<\operatorname{cf}(\mu)$ regular cardinals $\lambda_{i}$ such that

$$
\mu_{i}<\lambda_{i}<\mu, \quad \max \operatorname{pcf}\left(\left\{\lambda_{j}: j<i\right\}\right)<\lambda_{i},
$$

and there is an entangled sequence of linear orders each of cardinality $\lambda_{i}$ of length $\max \operatorname{pcf}\left(\left\{\lambda_{j}: j<i\right\}\right)$ (i.e., $\left.\operatorname{Ens}\left(\lambda_{i}, \max \operatorname{pcf}\left(\left\{\lambda_{j}: j<i\right\}\right)\right)\right)$. For some $\alpha, \lambda_{i}$ is defined if and only if $i<\alpha$. Clearly, $\alpha$ is a limit ordinal $\leq \operatorname{cf}(\mu)$, and $\lambda:=\operatorname{maxpcf}\left(\left\{\lambda_{j}: j<\alpha\right\}\right)>\mu$ [as otherwise $\lambda<\mu$ (as $\lambda$ is regular by $[\mathrm{Sh} 345 \mathrm{a}, 1.9,(1.1)])$, so there is $\lambda_{\alpha}$ as required among $\left.\left\{\left(\lambda+\mu_{\alpha}\right)^{+\gamma}: \gamma<\left(\lambda+\mu_{\alpha}\right)^{+4}\right\}\right]$. So clearly $\mu<\lambda=\operatorname{cf}(\lambda)<\operatorname{pp}^{+}(\mu)$ and by $3.3(2)$ there is an entangled linear order of cardinality $\lambda$ and density $\leq \sum_{i<\alpha} \max \operatorname{pcf}\left(\left\{\lambda_{j}: j<i\right\}\right) \leq \mu$. If $\lambda$ is a successor cardinal then we are done. Otherwise, clearly $\mu^{+\mu^{+4}} \leq \mathrm{pp}^{+}(\mu)$, and hence we can apply part (2).

(2) This follows by the claims below; each has the conclusion of $5.10(2)$ from assumptions which are not necessarily implied by the assumption of $5.10(2)$, but always at least one applies.

Claim 5.10.1. (1) Assume $\operatorname{cf}(\mu) \leq \kappa \leq \mu^{*}<\mu \leq 2^{\kappa}, \mu \leq \chi$ and $\chi^{+\kappa^{+4}} \leq$ $\operatorname{pp}_{\kappa}(\mu)$. Then there is $\lambda^{+} \in\left[\chi, \chi^{+\kappa^{+4}}\right]$ in which there is an entangled linear order $\mathcal{I}$ of density $\leq \mu$ but $\geq \mu^{*}$.

(2) If in addition $(\forall \alpha<\mu)\left(|\alpha|^{\kappa}<\mu\right)$ then we can add " $\mathcal{I}$ is $\operatorname{cf}(\mu)$ entangled".

(3) There is $\gamma<\kappa^{+4}$ and a set $\mathfrak{b} \subseteq \operatorname{Reg} \cap \mu \backslash \mu^{*}$ of $\left(\mu^{*}, \kappa^{+}, 2\right)$-inaccessible cardinals such that $|\mathfrak{b}| \leq \kappa, \mathfrak{b}$ is the disjoint union of $\mathfrak{b}_{\varepsilon}($ for $\varepsilon<\kappa), \sup \left(\mathfrak{b}_{\varepsilon}\right)$ is the same for all $\varepsilon<\kappa$ and $J_{\mathfrak{b}}^{\text {bd }} \subseteq J_{<\chi^{+\gamma+1}}[\mathfrak{b}]$ and $\chi^{+(\gamma+1)} \in \operatorname{pcf}\left(\mathfrak{b}_{\varepsilon}\right)$.

Proof. (1) Of course we can decrease $\mu$ as long as $\mu^{*}<\mu, \operatorname{cf}(\mu) \leq \kappa$ and $\chi^{+\kappa^{+4}} \leq \operatorname{pp}_{\kappa}(\mu)$. By [Sh 355, 2.3], without loss of generality we have

$$
\mathfrak{a} \subseteq\left(\mu^{*}, \mu\right) \cap \operatorname{Reg} \&|\mathfrak{a}| \leq \kappa \Rightarrow \max \operatorname{pcf}(\mathfrak{a})<\operatorname{pp}^{+}(\mu) .
$$

By induction on $i<\kappa$, we choose $\mathfrak{b}_{i}$ and $\gamma_{i}$ such that:

(i) $\mathfrak{b}_{i} \subseteq \operatorname{Reg} \cap \mu \backslash \bigcup_{j<i} \mathfrak{b}_{j} \backslash \mu^{*}$

(ii) $\gamma_{i}<\kappa^{+4}$ is a successor ordinal,

(iii) $\chi^{+\gamma_{i}} \in \operatorname{pcf}\left(\mathfrak{b}_{i}\right)$,

(iv) $\gamma_{i}$ is the first successor ordinal for which $\chi^{+\gamma_{i}} \notin \operatorname{pcf}\left(\bigcup_{j<i} \mathfrak{b}_{j}\right)$,

(v) all members of $\mathfrak{b}_{i}$ are $\left(\mu_{0}, \kappa^{+}, 2\right)$-inaccessible (i.e. $\theta \in \mathfrak{b}_{i} \& \mathfrak{a} \subseteq$ $\left.\left(\mu_{0}, \theta\right) \&|\mathfrak{a}| \leq \kappa \Rightarrow \max \operatorname{pcf}(\mathfrak{a})<\theta\right)$,

(vi) $\mathfrak{b}_{i}$ has cardinality $\leq \kappa$. 
Note that this is possible, since if $|\mathfrak{b}| \leq \kappa$ then $\operatorname{pcf}(\mathfrak{b})$ cannot contain the interval $\left[\chi, \chi^{+\kappa^{+4}}\right] \cap \operatorname{Reg}(\operatorname{see}[\operatorname{Sh} 410, \S 3])$. Let $\mathfrak{d}:=\left\{\chi^{+\gamma_{i}}: i<\kappa\right\}$, and let $\left\langle\mathfrak{b}_{\theta}[\mathfrak{d}]: \theta \in \operatorname{pcf}(\mathfrak{d})\right\rangle$ be as in $[\operatorname{Sh} 371,2.6]$. Note that we know $\operatorname{pcf}(\mathfrak{d}) \subseteq\left[\chi, \chi^{+\kappa^{+4}}\right]$ (by $[$ Sh 400, 4.2]). Let $\theta \in \operatorname{pcf}(\mathfrak{d})$ be minimal such that $\operatorname{otp}\left(\mathfrak{b}_{\theta}[\mathfrak{d}]\right) \geq \kappa$, so necessarily $\theta$ is a successor cardinal. Let $\left\langle\mathfrak{d}_{\alpha}: \alpha<\kappa\right\rangle$ be a partition of $\mathfrak{b}_{\theta}[\mathfrak{d}]$ to pairwise disjoint subsets of order type $\geq \kappa$. Let $\mathfrak{b}_{\alpha}^{\prime}=\bigcup\left\{\mathfrak{b}_{i}: \chi^{+\gamma_{i}} \in \mathfrak{d}_{\alpha}\right\}$ (for $\left.\alpha<\kappa\right)$ and $\mathfrak{a}=\bigcup_{\alpha<\kappa} \mathfrak{b}_{\alpha}^{\prime}$. Now we can finish by $3.2(1)$.

(2) In this case we can in the beginning increase $\mu^{*}\left(\right.$ still $\mu^{*}<\mu$ ) such that the second sentence of the proof of 5.10.1(1) holds. Necessarily $\sup \left(\mathfrak{b}_{i}\right)=\mu$ for each $i<\kappa$.

(3) Included in the proof above. $\mathbf{m}_{5.10 .1}$

Claim 5.10.2. If $\operatorname{cf}(\mu)<\kappa=\operatorname{cf}(\kappa)<\mu \leq \chi, \chi^{+\kappa^{+4}} \leq \operatorname{pp}(\mu)$ and $\mu$ is $\left(*, \kappa^{+}, 2\right)$-inaccessible then:

(1) We can find an increasing sequence $\left\langle\lambda_{i}: i<\operatorname{cf}(\mu)\right\rangle$ of regular cardinals with limit $\mu$ such that for each $i, \operatorname{Ens}\left(\lambda_{i}, \kappa\right)$ and $\operatorname{pcf}\left(\left\{\lambda_{i}: i<\operatorname{cf}(\mu)\right\}\right)$ has a member in $\left(\chi, \chi^{+\kappa^{+4}}\right)$ and $\lambda_{i}>\max \left(\operatorname{pcf}\left(\left\{\lambda_{j}: j<i\right\}\right)\right)$ and $\lambda_{i}>\kappa$.

(2) In addition, $\lambda_{i} \in \operatorname{pcf}_{\kappa}^{\mathrm{ex}}\left(\mathfrak{a}_{i}\right)$ for some sets $\mathfrak{a}_{i} \subseteq \operatorname{Reg} \cap \lambda_{i} \backslash \bigcup_{j<i} \lambda_{j}$ of $\left(\mu, \kappa^{+}, 2\right)$-inaccessible cardinals of cardinality $\kappa$. If $\operatorname{cf}(\mu)>\aleph_{0}$ then $\prod_{i<\operatorname{cf}(\mu)} \lambda_{i} / J_{\mathrm{cf}(\mu)}^{\mathrm{bd}}$ has true cofinality.

Proof. Choose $\mu^{*} \in(\kappa, \mu)$ such that

$$
\mu^{\prime} \in\left(\mu^{*}, \mu\right) \& \operatorname{cf}\left(\mu^{\prime}\right) \leq \kappa \Rightarrow \operatorname{pp}_{\kappa}\left(\mu^{\prime}\right)<\mu
$$

(exists, as $\mu$ is $\left(*, \kappa^{+}, 2\right.$ )-inaccessible; see [Sh 355, 2.3]). Let $\mathfrak{b}_{i}$ and $\gamma_{i}$ (for $i<\kappa)$ be as in the proof of 5.10.1; so $\min \left(\mathfrak{b}_{i}\right)>\mu^{*}$ and $\mathfrak{d}:=\left\{\chi^{+\gamma_{i}}: i<\kappa\right\}$ and $\left\langle\mathfrak{b}_{\theta}[\mathfrak{d}]: \theta \in \operatorname{pcf}(\mathfrak{d})\right\rangle$ are as in $[\operatorname{Sh} 371,2.6]$. Let $\theta \in \operatorname{pcf}(\mathfrak{d})$ be minimal such that $\operatorname{otp}\left(\mathfrak{b}_{\theta}[\mathfrak{d}]\right)=\kappa$. Without loss of generality $\mathfrak{b}_{\theta}[\mathfrak{d}]=\mathfrak{d}$, so $\theta=\max \operatorname{pcf}(\mathfrak{d})$. Note that $\theta \in \operatorname{pcf}(\mathfrak{d}) \subseteq\left(\chi, \chi^{+\kappa^{+4}}\right)$ is a successor cardinal. Let $\left\langle\mu_{i}: i<\operatorname{cf}(\mu)\right\rangle$ be strictly increasing continuous with limit $\mu$ with $\mu_{0}>\mu^{*}$. Let $\mathfrak{a}:=\bigcup_{i<\kappa} \mathfrak{b}_{i}$ and let $\left\langle\mathfrak{b}_{\sigma}[\mathfrak{a}]: \sigma \in \operatorname{pcf}(\mathfrak{a})\right\rangle$ be as in [Sh 371, 2.6]. For each $\varepsilon<\operatorname{cf}(\mu)$, we can find finite $\mathfrak{e}_{\varepsilon} \subseteq \operatorname{pcf}_{\kappa}^{\operatorname{ex}}\left(\mathfrak{a} \cap \mu_{\varepsilon}\right)$ and $\mathfrak{c}_{\varepsilon} \subseteq \mathfrak{a} \cap \mu_{\varepsilon},\left|\mathfrak{c}_{\varepsilon}\right|<\kappa$ such that $\mathfrak{a} \cap \mu_{\varepsilon} \subseteq \bigcup_{\sigma \in \mathfrak{e}_{\varepsilon}} \mathfrak{b}_{\sigma}[\mathfrak{a}] \cup \mathfrak{c}_{\varepsilon}$. As $\operatorname{cf}(\mu)<\kappa=\operatorname{cf}(\kappa)$, we can find $\zeta<\kappa$ such that

$$
(\forall \varepsilon<\operatorname{cf}(\mu))\left(\mathfrak{c}_{\varepsilon} \subseteq \bigcup_{i<\zeta} \mathfrak{b}_{i}\right) .
$$

So by renaming $\zeta=0$ (so $\mathfrak{c}_{\varepsilon}=\emptyset$ ). By 5.12(4) below, each $\sigma \in \mathfrak{e}_{\varepsilon}$ satisfies $\operatorname{Ens}(\sigma, \kappa)$. As in the proof of [Sh 371, 1.5] and [Sh 430, 6.5] one of the following holds:

$(*)_{1} \quad \operatorname{cf}(\mu)>\aleph_{0}$ and for some $S \subseteq \operatorname{cf}(\mu)$ unbounded with otp $(S)=\operatorname{cf}(\mu)$, and $\sigma_{\varepsilon} \in \mathfrak{e}_{\varepsilon}$ for $\varepsilon \in S$, we have $\theta=\operatorname{tcf}\left(\prod_{\varepsilon \in S} \sigma_{\varepsilon} / J_{S}^{\mathrm{bd}}\right)$, 
$(*)_{2} \quad \operatorname{cf}(\mu)=\aleph_{0}$ and for some increasing sequence $\left\langle\sigma_{\zeta}: \zeta<\operatorname{cf}(\mu)\right\rangle$ of regular cardinals from $\bigcup_{\varepsilon<\operatorname{cf}(\mu)} \mathfrak{e}_{\varepsilon}$ with limit $\mu$, and an ideal $I$ on $\omega$ extending $J_{c f(\mu)}^{\mathrm{bd}}$ we have $\theta=\operatorname{tcf}\left(\prod_{\zeta<\operatorname{cf}(\mu)} \sigma_{\zeta} / I\right)$.

If $(*)_{1}$ holds then (by the choice of $\mu_{0}$ ) without loss of generality $\varepsilon \in S \Rightarrow$ $\sigma_{\varepsilon}>\max \operatorname{pcf}\left(\left\{\sigma_{\zeta}: \zeta<\varepsilon\right\}\right)$, so we can apply 3.1. If $(*)_{2}$ holds, necessarily $\max \operatorname{pcf}\left(\left\{\sigma_{\zeta}: \zeta<\varepsilon\right\}\right)$ is $\sigma_{\varepsilon-1}$ so we can apply 3.1. In both cases we get the desired conclusion. $\mathbf{\square . 1 0 . 2}$

Claim 5.10.3. Assume that $\mu$ is a singular strong limit cardinal, or at least that it is $(*, \kappa, 2)$-inaccessible for every $\kappa<\mu, \operatorname{cf}(\mu)<\mu \leq \chi$ and $\chi^{+\mu^{+4}} \leq \operatorname{pp}(\mu)$.

(1) We can find $\lambda^{+} \in\left(\chi, \chi^{+\mu^{+4}}\right)$ in which there is an entangled linear order with density $\mu$.

(2) Moreover, we can find a strictly increasing sequence $\left\langle\lambda_{i}: i<\operatorname{cf}(\mu)\right\rangle$ with limit $\mu, \max \operatorname{pcf}\left(\left\{\lambda_{j}: j<i\right\}\right)<\lambda_{i}$ and $\lambda^{+}=\max \operatorname{pcf}\left(\left\{\lambda_{i}: i<\operatorname{cf}(\mu)\right\}\right)$. Letting $\kappa_{i}=\left(\sum_{j<i} \lambda_{j}+\mu^{*}\right)^{+}$we can also find a set $\mathfrak{a}_{i} \subseteq \operatorname{Reg} \cap \lambda_{i} \backslash \bigcup_{j<i} \lambda_{j}$ of $\left(\kappa_{j_{i}}^{+}, \kappa_{j_{i}}^{+}, 2\right)$-inaccessible cardinals of cardinality $\kappa_{j_{i}}$ such that $\lambda_{i} \in \operatorname{pcf}_{\kappa_{i}}^{\mathrm{ex}}\left(\mathfrak{a}_{i}\right)$ and $j_{i} \leq i$. Also $\operatorname{cf}(\mu)>\aleph_{0}$ implies $\lambda=\operatorname{tcf}\left(\prod_{i<\operatorname{cf}(\mu)} \lambda_{i} / J_{\operatorname{cf}(\mu)}^{\mathrm{bd}}\right)$ and $(\forall i<$ $\operatorname{cf}(\mu))\left(j_{i}=i\right)$.

P r o of. Choose $\left\langle\mu_{i}: i<\operatorname{cf}(\mu)\right\rangle$ strictly increasing continuous with limit $\mu$. By induction on $\varepsilon<\operatorname{cf}(\mu)$ we choose $\theta^{\varepsilon} \in\left(\chi, \chi^{+\mu^{+4}}\right)$ and $\left\langle\lambda_{\zeta}^{\varepsilon}: \zeta<\operatorname{cf}(\mu)\right\rangle$ as follows: arriving at $\varepsilon$ we apply the proof of 5.10 .2 to $\kappa_{\varepsilon}=\mu_{\varepsilon}^{+}$and $\left({ }^{7}\right)$ $\chi_{\varepsilon}=\sup \left(\left\{\theta_{\zeta}: \zeta<\varepsilon\right\} \cup\{\chi\}\right)$ and get $\left\langle\lambda_{\zeta}^{\varepsilon}: \zeta<\operatorname{cf}(\mu)\right\rangle$ as there. So there is a successor $\theta_{\varepsilon} \in \operatorname{pcf}\left(\left\{\lambda_{\zeta}^{\varepsilon}: \zeta<\operatorname{cf}(\mu)\right\}\right) \cap\left[\chi_{\varepsilon}, \chi_{\varepsilon}^{\kappa_{\varepsilon}^{+4}}\right)$ such that $\theta_{\varepsilon}=$ $\max \operatorname{pcf}\left(\left\{\lambda_{\zeta}^{\varepsilon}: \zeta<\operatorname{cf}(\mu)\right\}\right)$. Hence $\theta_{\varepsilon}>\bigcup_{\zeta<\varepsilon} \theta_{\zeta}$ and $\chi<\theta_{\varepsilon}<\chi^{+\mu^{+4}}$, and without loss of generality $\mu_{i}^{+4}<\mu_{i+1}$. Let $\chi^{+\gamma_{\varepsilon}}$ be $\theta_{\varepsilon}, \mathfrak{b}_{\varepsilon}:=\left\{\lambda_{\zeta}^{\varepsilon}: \zeta<\operatorname{cf}(\mu)\right\}$ (for $\varepsilon<\operatorname{cf}(\mu)), \theta=\max \operatorname{pcf}\left(\left\{\theta_{\varepsilon}: \varepsilon<\operatorname{cf}(\mu)\right\}\right), \mathfrak{a}:=\bigcup\left\{\mathfrak{b}_{\varepsilon}: \varepsilon<\operatorname{cf}(\mu)\right\}$ and without loss of generality

$$
J_{<\theta}\left[\left\{\theta_{\varepsilon}: \varepsilon<\operatorname{cf}(\mu)\right\}\right] \subseteq\left\{\left\{\theta_{\varepsilon}: \varepsilon \in a\right\}: a \subseteq \operatorname{cf}(\mu),|a|<\operatorname{cf}(\mu)\right\} .
$$

Note that

$$
\begin{aligned}
\operatorname{pcf}\left(\left\{\theta_{\varepsilon}: \varepsilon<\operatorname{cf}(\mu)\right\}\right) & \subseteq \operatorname{pcf}\left(\left\{\chi^{+\gamma+1}: \gamma<\mu^{+4}\right\}\right) \\
& \subseteq \operatorname{Reg} \cap\left[\chi, \chi^{+\mu^{+4}}\right] \cap\left(\chi, \operatorname{pp}^{+}(\mu)\right),
\end{aligned}
$$

so each member of $\operatorname{pcf}\left(\left\{\theta_{\varepsilon}: \varepsilon<\operatorname{cf}(\mu)\right\}\right)$ is a successor cardinal. Let $\left\langle\mathfrak{b}_{\sigma}[\mathfrak{a}]\right.$ : $\sigma \in \operatorname{pcf}(\mathfrak{a})\rangle$ be as in $[\operatorname{Sh} 371,2.6]$.

First assume $\operatorname{cf}(\mu)>\aleph_{0}$. For every limit $\varepsilon<\operatorname{cf}(\mu)$ let $\mathfrak{e}_{\varepsilon}$ be a finite subset of $\operatorname{pcf}\left(\mathfrak{a} \cap \mu_{\varepsilon}\right)$ such that:

$\left({ }^{7}\right)$ We could have asked $\chi_{\varepsilon}=\max \left\{\max \operatorname{pcf}\left(\left\{\theta_{\zeta}: \zeta<\varepsilon\right\}\right), \chi\right\}$ and thus later "the $\left\{\lambda_{\zeta}^{\varepsilon}: \zeta<\operatorname{cf}(\mu)\right\}$ are pairwise disjoint" (while omitting "few" $\lambda_{\zeta}^{\varepsilon}$ for each $\varepsilon$ ). 
$(\alpha)$ for some $\zeta_{\varepsilon}<\varepsilon$,

$$
\left(\mathfrak{a} \cap \mu_{\varepsilon}\right) \backslash \bigcup_{\sigma \in \mathfrak{e}_{\varepsilon}} b_{\sigma}[\mathfrak{a}] \subseteq\left\{\lambda_{i}^{\xi}: i<\operatorname{cf}(\mu) \text { and } \lambda_{i}^{\xi}<\mu_{\zeta_{\varepsilon}}\right\},
$$

$(\beta)$ for every $\zeta<\varepsilon$ and for every $\sigma \in \mathfrak{e}_{\varepsilon}$,

$$
\sigma \in \operatorname{pcf}\left(\left\{\lambda_{i}^{\xi}: i<\operatorname{cf}(\mu) \& \mu_{\zeta}<\lambda_{i}^{\xi}<\mu_{\varepsilon}\right\}\right) .
$$

(Exist by [Sh 430, 6.7, 6.7A-F].)

So for every $\sigma \in \mathfrak{e}_{\varepsilon}$, by 3.3(2), there is an entangled linear order of cardinality $\sigma$ (remember $\operatorname{Ens}\left(\lambda_{\zeta}^{\varepsilon}, \mu_{\varepsilon}^{+}\right)$and the choice of $\left\langle\lambda_{\zeta}^{\varepsilon}: \zeta<\operatorname{cf}(\mu)\right\rangle$, $\kappa_{\varepsilon}^{+}$). Also, by [Sh:g, VIII, $\left.\S 1\right]$ (more in [Sh 430, 6.7, 6.7A-F) for some unbounded set $S \subseteq \operatorname{cf}(\mu)$ and $\sigma_{\varepsilon} \in \mathfrak{e}_{\varepsilon}$ we have $\theta=\operatorname{tcf}\left(\prod_{\varepsilon \in S} \sigma_{\varepsilon} / J_{S}^{\text {bd }}\right)$. Note that without loss of generality $\sigma_{\varepsilon}>\prod_{\zeta<\varepsilon} \sigma_{\zeta}$ (when $\mu$ is strong limit!) or at least $\sigma_{\varepsilon}>\max \operatorname{pcf}\left(\left\{\sigma_{\zeta}: \zeta<\varepsilon\right\}\right)$, so by $3.3(2)$ we can get the desired conclusion.

Now assume $\operatorname{cf}(\mu)=\aleph_{0}$. Use [Sh 430, 6.7] to find finite $\mathfrak{e}_{\varepsilon} \subseteq \operatorname{pcf}\left(\mathfrak{a} \cap \mu_{\varepsilon}\right)$ for $\varepsilon<\omega$ such that

$$
\varepsilon<\xi<\omega \Rightarrow \max \left(\mathfrak{e}_{\varepsilon} \cup\left\{\aleph_{0}\right\}\right)<\min \left(\mathfrak{e}_{\zeta} \cup\{\mu\}\right)
$$

(the $\left\{\aleph_{0}\right\},\{\mu\}$ are for empty $\mathfrak{e}_{n}$ 's), and so $\operatorname{otp}(\mathfrak{e})=\omega, \sup (\mathfrak{e})=\mu$ where $\mathfrak{e}=\bigcup_{\varepsilon} e_{\varepsilon}$ and $\mathfrak{a} \cap \mu_{\varepsilon}=\bigcup\left\{\mathfrak{b}_{\sigma}[\mathfrak{a}]: \sigma \in \bigcup_{n \leq \varepsilon} \mathfrak{e}_{n}\right\}$; hence $\sigma \in \operatorname{pcf}(\mathfrak{e})$. Define $h: \operatorname{pcf}(\mathfrak{a}) \rightarrow \omega$ by $h(\sigma)=\max \left\{n<\omega: \operatorname{Ens}\left(\sigma, \mu_{n}\right)\right.$ or $\left.n=0\right\}$. By 3.4(2) it suffices to prove, for each $n<\omega$, that $\{\sigma \in \mathfrak{e}: h(\sigma) \leq n\} \in J_{<\sigma}[\mathfrak{e}]$. This can be easily checked. $\mathbf{m} 5.10 .3$

(3), (4) Left to the reader. -5.10

Remark 5.11. (1) Under the assumptions of 5.10 .2 we can get

$(\circledast) \quad$ there are a successor cardinal $\lambda^{+} \in\left(\chi, \chi^{+\mu^{+4}}\right)$ and an increasing sequence $\bar{\lambda}=\left\langle\lambda_{i}: i<\delta\right\rangle$ with limit $\mu$ such that $\delta<\kappa^{+}, \lambda^{+}=$ $\operatorname{tcf}\left(\prod_{i<\delta} \lambda_{i} / J\right), J$ an ideal on $\delta$ extending $J_{\delta}^{\mathrm{bd}}, \lambda_{i}$ is $\left(\mu^{*}, \kappa^{+}, 2\right)$ inaccessible (where $\mu^{*}=\min \left\{\mu^{\prime}: \mu^{*}\right.$ is $\left(\mu^{\prime}, \kappa^{+}, 2\right)$-inaccessible $\}$ ) and there is an entangled linear order of cardinality $\lambda^{+}$.

(2) In the proof of 5.10 .3 we can have $\mathfrak{a}=\bigcup_{i<\operatorname{cf}(\mu)} \mathfrak{a}_{i}, \mu=\sup \left(\mathfrak{a}_{i}\right)$ for each $i<\operatorname{cf}(\mu)$, and let $\mathfrak{a}=\left\{\lambda_{\zeta}^{\varepsilon}: \varepsilon, \zeta<\operatorname{cf}(\mu)\right\}$,

$$
\mathfrak{a}_{i}=\left\{\lambda_{\zeta}^{\varepsilon} \in \mathfrak{a}: \varepsilon \in \mathfrak{a}_{i} \text { but for no } \xi<\varepsilon \text { does } \lambda_{\zeta}^{\varepsilon} \in\left\{\lambda_{j}^{\xi}: j<\operatorname{cf}(\mu)\right\}\right\}
$$

and apply 3.2(1).

Proposition 5.12. Let $\left\langle\mathfrak{b}_{\theta}[\mathfrak{a}]: \theta \in \operatorname{pcf}(\mathfrak{a})\right\rangle$ be as in $[$ Sh $371,2.6]$.

(1) If $|\mathfrak{a}| \geq \kappa($ and $|\mathfrak{a}|<\min (\mathfrak{a}))$ then $\operatorname{pcf}_{\kappa}^{\text {ex }}(\mathfrak{a})$ has a last element.

(2) Assume that $\theta=\max \operatorname{pcf}(\mathfrak{a}), \mathfrak{c} \subseteq \theta \cap \operatorname{pcf}(\mathfrak{a}),|\mathfrak{c}|<\min (\mathfrak{c})$ and

$$
\mathfrak{b} \in J_{<\theta}[\mathfrak{a}] \Rightarrow \mathfrak{c} \cap \operatorname{pcf}(\mathfrak{a} \backslash \mathfrak{b}) \neq \emptyset .
$$

Then $\theta \in \operatorname{pcf}(\mathfrak{c})$. 
(3) If $\mathfrak{a}=\bigcup_{i<\sigma} \mathfrak{a}_{i}$ and $\sigma<\operatorname{cf}(\kappa)$ and $\theta \in \operatorname{pcf}_{\kappa}^{\text {ex }}(\mathfrak{a})$ then we can find finite $\mathfrak{e}_{i} \subseteq \operatorname{pcf}_{\kappa}^{\text {ex }} \mathfrak{a}_{i}$ for $i<\sigma$ such that $\left|\mathfrak{a}_{i} \backslash \bigcup_{\lambda \in \mathfrak{e}_{i}} \mathfrak{b}_{\lambda}\left[\mathfrak{a}_{i}\right]\right|<\kappa$ and $\theta \in \operatorname{pcf}\left(\bigcup_{i<\sigma} \mathfrak{e}_{i}\right)$. $\left[\right.$ And if $\operatorname{cf}(\sigma)>\aleph_{0}, \mathfrak{a}_{i}$ is increasing with $i$ and $S \subseteq \sigma=\sup S$ then $\theta \in$ $\operatorname{pcf}\left(\bigcup_{i \in S} \mathfrak{e}_{i}\right)$.]

(4) Assume $\chi<\min (\mathfrak{a})$, and each $\mu \in \mathfrak{a}$ is $(\chi, \kappa, 2)$-inaccessible and $\kappa>\aleph_{0}$. If $\theta \in \operatorname{pcf}_{\kappa}^{\text {ex }}(\mathfrak{a})$ then $\operatorname{Ens}\left(\theta, 2^{\kappa}\right)$ holds, exemplified by linear orders of density $>\chi$.

Proof. (1) Among the sets $\mathfrak{c} \subseteq \mathfrak{a}$ of cardinality $<\kappa$ choose one with $\max \operatorname{pcf}(\mathfrak{a} \backslash \mathfrak{c})$ minimal. So $\max \operatorname{pcf}(\mathfrak{a} \backslash \mathfrak{c})=\max _{\operatorname{pcf}}{ }_{\kappa}^{\text {ex }}(\mathfrak{a})$.

(2) By [Sh 345a, 1.16].

(3) Easy, as in [Sh 371, §1].

(4) Without loss of generality $\theta=\max \operatorname{pcf}(\mathfrak{a})$, $\mathfrak{a}$ has no last element and

$$
\mu \in \mathfrak{a} \Rightarrow \theta \notin \operatorname{pcf}_{\kappa}^{\mathrm{ex}}(\mathfrak{a} \cap \mu),
$$

so $J_{\mathfrak{a}}^{\mathrm{bd}} \subseteq J_{<\theta}^{\mathrm{ex}, \kappa}[\mathfrak{a}]$ (see Definition 5.9). We are going to prove the statement by induction on $\theta$.

If $\mathfrak{a}\left(=\mathfrak{b}_{\theta}[\mathfrak{a}]\right)$ can be divided into $\kappa$ sets, no one of which is in $J_{<\theta}[\mathfrak{a}]+J_{\mathfrak{a}}^{\text {bd }}$, this should be clear (use e.g. 3.1(1): there are such $A_{i}$ by [EK], see e.g. [Sh g, Appendix]).

Also, if there are $\mathfrak{c} \subseteq \theta \cap \operatorname{pcf}_{\kappa}^{\text {ex }}(\mathfrak{a})$ (such that $\left.|\mathfrak{c}|<\min (\mathfrak{c})\right)$ and an ideal $I$ on $\mathfrak{c}$ satisfying $\theta=\operatorname{tcf}\left(\prod \mathfrak{c} / I\right)$, then we can find $\mathfrak{d} \subseteq \theta \cap \operatorname{pcf}(\mathfrak{c})$ such that $(\forall \sigma \in \mathfrak{d})(\max \operatorname{pcf}(\mathfrak{d} \cap \sigma)<\sigma), \theta=\max \operatorname{pcf}(\mathfrak{d})$ and then use the induction hypothesis and 3.4(1). So by part (1) (of 5.12) the remaining case is:

$$
\text { if } \mathfrak{c} \subseteq \theta \cap \operatorname{pcf}_{\kappa}^{\text {ex }}(\mathfrak{a}) \text { and }|\mathfrak{c}|<\min (\mathfrak{c}) \text { then } \theta \notin \operatorname{pcf}(\mathfrak{c}) .
$$

Without loss of generality

$$
\mathfrak{a}^{\prime} \subseteq \mathfrak{a} \& \theta \in \operatorname{pcf}_{\kappa}^{\text {ex }}\left(\mathfrak{a}^{\prime}\right) \Rightarrow \sup \left(\theta \cap \operatorname{pcf}_{\kappa}^{\text {ex }}\left(\mathfrak{a}^{\prime}\right)\right)=\sup \left(\theta \cap \operatorname{pcf}_{\kappa}^{\text {ex }}(\mathfrak{a})\right)
$$

We can try to choose, by induction on $i, \theta_{i} \in \operatorname{pcf}_{\kappa}^{\text {ex }}(\mathfrak{a})$ such that $\theta>\theta_{i}>$ $\max \operatorname{pcf}\left(\left\{\theta_{j}: j<i\right\}\right)$. By localization (see [Sh 371, §3]) we cannot have $\left\langle\theta_{i}\right.$ : $\left.i<|\mathfrak{a}|^{+}\right\rangle\left(\operatorname{as} \max \operatorname{pcf}\left(\left\{\theta_{i}: i<|\mathfrak{a}|^{+}\right\}\right) \in \operatorname{pcf}\left(\left\{\theta_{i}: i<\alpha\right\}\right)\right.$ for some $\alpha<|\mathfrak{a}|^{+}$, hence $\theta_{\alpha}<\theta_{\alpha+1}<\max \operatorname{pcf}\left(\left\{\theta_{i}: i<|\mathfrak{a}|^{+}\right\} \leq \max \operatorname{pcf}\left(\left\{\theta_{i}: i<\alpha\right\}\right)=\theta_{\alpha}\right.$, a contradiction). So for some $\alpha<|\mathfrak{a}|^{+}, \theta_{i}$ is defined if and only if $i<\alpha$. If $\max \operatorname{pcf}\left(\left\{\theta_{i}: i<\alpha\right\}\right)<\theta$ we can get a contradiction with ( $\boxplus$ ) (by 5.12(1)). If the equality holds, we get a contradiction with $(\otimes)$. $\mathbf{m}_{5.12}$

We want to state explicitly the pcf theorems behind 5.10.

Proposition 5.13. Assume $\mu$ is a singular cardinal which is a fix point and $\mu_{0}<\mu$.

(1) There are $\lambda$ and $\left\langle\left(\lambda_{i}, \bar{\lambda}^{i}\right): i<\delta\right\rangle$ such that:

(a) $\left\langle\lambda_{i}: i<\delta\right\rangle$ is a strictly increasing sequence of regular cardinals, $\delta$ is a limit ordinal $\leq \operatorname{cf}(\mu), \lambda_{i} \in \operatorname{Reg} \cap \mu \backslash \mu_{0}, \lambda_{i}^{*}=\max \operatorname{pcf}\left(\left\{\lambda_{j}:\right.\right.$ 
$j<i\})<\lambda_{i}, \lambda \in \operatorname{Reg} \cap\left(\mu, \operatorname{pp}^{+}(\mu)\right)$ and $\lambda=\operatorname{tcf}\left(\prod_{i<\delta} \lambda_{i} / J_{\delta}^{\mathrm{bd}}\right)$,

(b) $\bar{\lambda}^{i}=\left\langle\lambda_{j}^{i}: j<\left(\lambda_{i}^{*}\right)^{+}\right\rangle$is strictly increasing, $\lambda_{j}^{i} \in \operatorname{Reg} \cap \lambda_{i} \backslash \lambda_{i}^{*}$, $\lambda_{i}=\operatorname{tcf}\left(\prod_{j} \lambda_{j}^{i} / J_{\left(\lambda_{j}^{*}\right)^{+}}^{\mathrm{bd}}\right)$ and $\lambda_{j}^{i}>\max \operatorname{pcf}\left(\left\{\lambda_{\zeta}^{i}: \zeta<j\right\}\right)$.

(2) Assume in addition $\mu \leq \chi_{0}$ and $\chi_{0}^{+\mu^{+4}} \leq \mathrm{pp}^{+}(\mu)$. Then for some $\gamma<\mu^{+4}$, letting $\lambda=\chi^{+\gamma+1}$, we can find a strictly increasing sequence $\left\langle\lambda_{i}: i<\delta\right\rangle$ of regular cardinals of length $\delta=\operatorname{cf}(\delta)<\operatorname{cf}(\mu)$ with $\lambda_{i} \in \operatorname{Reg} \cap$ $\mu \backslash \mu^{*}, \lambda_{i}>\operatorname{maxpcf}\left(\left\{\lambda_{j}: j<i\right\}\right), \lambda=\max \operatorname{pcf}\left(\left\{\lambda_{i}: i<\delta\right\}\right)$, and letting $\kappa_{i}=\left(\sum_{j<i} \lambda_{j}+\mu^{*}\right)^{+}$we can also find sets $\mathfrak{a}_{i} \subseteq \operatorname{Reg} \cap \lambda_{i} \backslash \bigcup_{j<i} \lambda_{j} \backslash \mu^{*}$ of $\left(\kappa_{j_{i}}, \kappa_{j_{i}}^{+}, 2\right)$-inaccessible cardinals of cardinality $\kappa_{j_{i}}$ such that $\lambda_{i} \in \operatorname{pcf}_{\kappa_{i}}^{\mathrm{ex}}\left(\mathfrak{a}_{i}\right)$, $j_{i} \leq i$. Also if $\operatorname{cf}(\mu)>\aleph_{0}$ then $\lambda=\operatorname{tcf}\left(\prod_{i<\operatorname{cf}(\mu)} \lambda_{i} / J_{\mathrm{cf}(\mu)}^{\mathrm{bd}}\right)$.

(3) If part (2) does not apply then in (1), $\lambda$ is a successor.

Proof. Let $\left\langle\mu_{i}: 1 \leq i<\operatorname{cf}(\mu)\right\rangle$ be increasing continuous with limit $\mu$, $\mu_{1}>\mu_{0}$ and without loss of generality if $\mu$ is $(*, \operatorname{cf}(\mu), 2)$-inaccessible then

$$
\mu^{\prime} \in\left[\mu_{0}, \mu\right) \& \operatorname{cf}\left(\mu^{\prime}\right) \leq \operatorname{cf}(\mu) \Rightarrow \operatorname{pp}\left(\mu^{\prime}\right)<\mu,
$$

and hence

$\mathfrak{a} \subseteq\left[\mu_{0}, \mu\right) \cap \operatorname{Reg} \&|\mathfrak{a}| \leq \operatorname{cf}(\mu) \& \sup (\mathfrak{a})<\mu \Rightarrow \max \operatorname{pcf}(\mathfrak{a})<\mu$.

(1) Try to choose, by induction on $i<\operatorname{cf}(\mu), \lambda_{i}$ and $\mathfrak{a}_{i}, \lambda_{i}, \bar{\lambda}^{i}, \lambda_{i}^{+}, j_{i}$ such that:

$(\alpha)\left\{\lambda_{j}^{i}: j\right\} \cup\left\{\lambda_{i}, \lambda_{i}^{*}\right\} \subseteq\left(\mu_{j_{i}}, \lambda\right) \cap \operatorname{Reg}$,

( $\beta) \mu>\mu_{j_{i}}$

$(\gamma) \lambda_{i}^{*}, \bar{\lambda}^{i}, \lambda_{i}$ are as required in (1).

So for some $\alpha,\left(j_{i}, \lambda_{i}^{*}, \bar{\lambda}^{i}, \lambda_{i}\right)$ is defined if and only if $i<\alpha$; in fact, $\alpha$ is limit and $\operatorname{pcf}\left(\left\{\lambda_{i}: i<\alpha\right\}\right) \nsubseteq \mu$ (as in the proof of 5.10.1). For some unbounded set $A \subseteq \delta$ we have $\max \operatorname{pcf}\left(\left\{\lambda_{i}: i \in A\right\}\right)>\mu$ but it is minimal under this restriction. Now restricting ourselves to $A$ (and renaming) we finish.

(2) It follows from 5.10.1, 5.10.3.

(3) Should be clear. $\mathbf{m}_{5.13}$

Let us finish this section with stating some results which will be developed and presented with all details in a continuation of the present paper.

Proposition 5.14. Assume that

(A) (a) $T \subseteq \bigcup_{j \leq \delta} \prod_{i<j} \lambda_{i}$,

(b) $T$ is closed under initial segments,

(c) $T_{j}:=T \cap \prod_{i<j} \lambda_{i} \neq \emptyset$ for $j \leq \delta$,

(d) for $j<\delta$ and $\eta \in T_{j}$ we have $\left(\exists^{\lambda_{j}} \alpha<\lambda_{j}\right)(\eta \frown\langle\alpha\rangle \in T)$,

(e) $\left|T_{\delta}\right|=\kappa \geq \mu=\operatorname{cf}(\mu)>\sum_{i<\delta}\left|T_{i}\right|>\sigma$,

(f) for $\eta \in T_{i}, I_{\eta}$ is a $\sigma$-complete ideal on $\lambda_{i}$,

(g) $\sigma$ is a regular cardinal and $\operatorname{cf}(\delta) \geq \sigma$, 
(B) (a) $J$ is an ideal on $\delta$ extending $J_{\delta}^{\mathrm{bd}}$,

(b) $g_{i}$ is a function from $T_{i}$ to $\kappa_{i}$,

(c) for $i<\delta$ and $\alpha<\kappa_{i}$, I $I_{\alpha}^{i}$ is a $\sigma$-complete ideal on $\lambda_{i}$,

(d) if $\left\langle\eta_{\beta, \varepsilon}: \varepsilon<\varepsilon^{*}, \beta<\mu\right\rangle$ are pairwise distinct members of $T_{\delta}$, $\varepsilon^{*}<\sigma, i(*)<\delta,(\forall \beta<\mu)\left(\eta_{\varepsilon}=\eta_{\beta, \varepsilon} \backslash i(*)\right)$ for each $\varepsilon<\varepsilon^{*}$, and $\left\langle\eta_{\varepsilon}: \varepsilon<\varepsilon^{*}\right\rangle$ are pairwise distinct, then for some $A \in J$, for every $i \in \delta \backslash A$ there are $\nu_{\varepsilon} \in T_{i}$ for $\varepsilon<\varepsilon^{*}$ such that:

$(\alpha)\left\langle g_{i}\left(\nu_{\varepsilon}\right): \varepsilon<\varepsilon^{*}\right\rangle$ are pairwise distinct,

( $\beta$ ) for every $B_{\nu_{\varepsilon}} \in I_{g_{i}\left(\nu_{\varepsilon}\right)}^{i}\left(\right.$ for $\left.\varepsilon<\varepsilon^{*}\right)$, for some $\beta<\mu$ we have

$$
\left(\forall \varepsilon<\varepsilon^{*}\right)\left(\nu_{\varepsilon} \triangleleft \eta_{\beta, \varepsilon}\right) \quad \text { and } \quad \eta_{\beta, \varepsilon}(i) \notin B_{\nu_{\varepsilon}},
$$

(C) for $i<\delta,\left\langle\mathcal{I}_{\zeta}^{i}: \zeta<\kappa_{i}\right\rangle$ is a sequence of linear orders with universe $\lambda_{i}$ such that if $\varepsilon(*)<\sigma,\left\langle\zeta_{\varepsilon}: \varepsilon<\varepsilon(*)\right\rangle$ is a sequence of distinct members of $\kappa_{1},\left\langle\alpha_{\beta, \varepsilon}: \beta<\beta(*), \varepsilon<\varepsilon(*)\right\rangle$ is a sequence of ordinals $<\lambda$ such that

$$
\left(\forall \bar{B} \in \prod_{\varepsilon<\varepsilon(*)} \mathcal{I}_{\zeta_{\varepsilon}}\right)\left(\exists \beta<\beta^{*}\right)(\forall \varepsilon<\varepsilon(*))\left(\alpha_{\beta, \varepsilon} \notin B_{\varepsilon}\right)
$$

and $\varepsilon(*)=u \cup v, u \cap v=\emptyset$, then for some $\beta_{1}, \beta_{2}<\beta(*)$ we have

$$
\begin{aligned}
& \varepsilon \in u \Rightarrow \mathcal{I}_{\zeta_{\varepsilon}}^{i} \models \text { " } \alpha_{\beta_{1}, \varepsilon}<\alpha_{\beta_{2}, \varepsilon} ", \\
& \varepsilon \in v \Rightarrow \mathcal{I}_{\zeta_{\varepsilon}}^{i} \models \text { " } \alpha_{\beta_{1}, \varepsilon}>\alpha_{\beta_{2}, \varepsilon} "
\end{aligned}
$$

Then there is a $(\mu, \sigma)$-entangled linear order of cardinality $\lambda$.

Remark 5.15. (1) The proof is derived from the proof of 3.3 (and so from [Sh 355, 4.10]).

(2) Are the assumptions reasonable? At least they are not so rare, see [Sh 430, §5].

Proof of Proposition 5.14. The desired linear order $\mathcal{I}$ has the universe $T_{\delta}$ (which has cardinality $\lambda$ ) with the order: $\eta<_{\mathcal{I}} \nu$ if and only if for the minimal $i<\delta$ for which $\eta(i) \neq \nu(i)$ we have

$$
\mathcal{I}_{g(\eta\lceil i)} \models \text { " } \eta(i)<\nu(i) " .
$$

Details, as said before, will be presented somewhere else, but they should be clear already. 5.14

Proposition 5.16. We can replace $(\mathrm{B})(\mathrm{d})$ of 5.14 by

(d1) if $\left\langle\eta_{\beta, \varepsilon}: \varepsilon<\varepsilon^{*}, \beta<\mu\right\rangle$ are pairwise distinct members of $T_{\delta}, \varepsilon^{*}<\sigma$, $i(*)<\delta$ and

$$
(\forall \varepsilon<\varepsilon(*))(\forall \beta<\mu)\left(\eta_{\varepsilon}=\eta_{\beta, \varepsilon}\lceil i(*))\right.
$$

and $\left\langle\eta_{\varepsilon}: \varepsilon<\varepsilon^{*}\right\rangle$ are pairwise distinct, then for some $\beta<\mu$, $\left\{i<\delta:\right.$ for every $\bar{B} \in \prod_{\varepsilon<\varepsilon^{*}} I_{g_{i}\left(\eta_{\beta, \varepsilon} \backslash i\right)}$, for some $\gamma<\mu$ we have 


$$
\left(\forall \varepsilon<\varepsilon^{*}\right)\left(\eta_{\gamma, \varepsilon}\left\lceil i=\eta_{\beta, \varepsilon}\lceil i) \text { and }\left(\forall \varepsilon<\varepsilon^{*}\right)\left(\eta_{\gamma, \varepsilon}(i) \in B_{\varepsilon}\right)\right\}=\delta \bmod J\right.
$$

(d2) if $\eta_{\varepsilon} \in T_{\delta}\left(\varepsilon<\varepsilon^{*}<\sigma\right)$ are distinct, then

$\left\{i<\delta:\left\langle g_{i}\left(\eta_{\varepsilon}\lceil i): \varepsilon<\varepsilon^{*}\right\rangle\right.\right.$ is with no repetition $\} \neq \emptyset \bmod J$.

Proposition 5.17. Assume that:

(A) $\lambda=\operatorname{tcf}\left(\prod_{i<\delta} \lambda_{i} / J\right), \lambda_{i}>\theta_{i}:=\max \operatorname{pcf}\left(\left\{\lambda_{j}: j<i\right\}\right)$,

(B) $J$ a $\sigma$-complete ideal on the limit ordinal $\delta, \lambda_{i}=\operatorname{cf}\left(\lambda_{i}\right)>\delta$,

(C) $g_{i, j}: \theta_{i} \rightarrow \kappa_{i, j}$ for $j<j_{i}$ are such that for any $w \in\left[\theta_{i}\right]^{<\sigma}$ for some $j<j_{i}$ the restriction $g_{i, j}\lceil w$ is one-to-one,

(D) $\bar{a}=\left\langle a_{i}: i<\delta\right\rangle, a_{i} \subseteq\left(\bigcup_{\zeta<i}\{\zeta\} \times j_{\zeta}\right)$ are such that for any $\zeta$ and $j<j_{\zeta}$ we have $\left\{i:\left(\zeta, j_{\zeta}\right) \in a_{i}\right\} \neq \emptyset \bmod J$,

(E) $\operatorname{Ens}_{\sigma}\left(\lambda_{i}, \prod_{(\zeta, j) \in a_{i}} \kappa_{\zeta, j}\right)$.

Then for some $T$ the assumptions of 5.14 hold for $\mu_{i}=\lambda_{i}$ and $\kappa_{i}:=$ $\prod_{(\zeta, j) \in a_{i}} \kappa_{\zeta, j}$

Proposition 5.18. In 5.17, (C) + (D) + (E) holds if:

$\left(\mathrm{C}^{\prime}\right) \operatorname{Ens}_{\sigma}\left(\lambda_{i}, \kappa\right)$,

$\left(\mathrm{D}^{\prime}\right)(\alpha) \kappa^{|\delta|} \geq \sum_{i<\delta} \lambda_{i}$ but for $i<\delta, \kappa^{|i|}=\kappa($ so $\delta$ is a regular cardinal $)$ or

$(\beta) \kappa^{|\delta|} \geq \sum_{i<\delta} \lambda_{i}$ and there is a regular ultrafilter $E$ on $\delta$ disjoint from

$$
J \cup\{A \subseteq \delta: \operatorname{otp}(A)<\delta\} .
$$

Proposition 5.19. Suppose (A), (B) as in 5.14 and

(C) there is a sequence $\left\langle\mathcal{I}_{\zeta}^{i}: \zeta<\kappa_{i}\right\rangle$ of partial orders with universe $\lambda_{i}$ such that if $\varepsilon(*)<\sigma,\left\langle\zeta_{\varepsilon}: \varepsilon<\varepsilon(*)\right\rangle$ is a sequence of ordinals $<\kappa_{i}$ with no repetitions, $\left\langle\alpha_{\beta, \varepsilon}: \beta<\beta(*), \varepsilon<\varepsilon(*)\right\rangle$ is a sequence of ordinals $<\lambda$ such that

$$
\left(\forall \bar{B} \in \prod_{\varepsilon<\varepsilon(*)} \mathcal{I}_{\zeta_{\varepsilon}}\right)\left(\exists \beta<\beta^{*}\right)(\forall \varepsilon<\varepsilon(*))\left(\alpha_{\beta, \varepsilon} \notin B_{\varepsilon}\right)
$$

and $\varepsilon(*)=u \cup v, u \cap v=\emptyset$, then for some $\beta_{1}, \beta_{2}<\beta(*)$ we have

$$
\begin{aligned}
& \varepsilon \in u \Rightarrow \mathcal{I}_{\zeta_{\varepsilon}}^{i} \models \text { " } \alpha_{\beta, \varepsilon}<\alpha_{\beta_{2}, \varepsilon} ", \\
& \varepsilon \in v \Rightarrow \mathcal{I}_{\zeta_{\varepsilon}}^{i} \models \text { " } \neg \alpha_{\beta, \varepsilon}<\alpha_{\beta_{2}, \varepsilon} " .
\end{aligned}
$$

Then there is a Boolean algebra $B$ with $|B|=\lambda$ and with neither chain nor pie of cardinality $\lambda$; moreover, for $\varepsilon<\sigma, B^{\sigma}$ has those properties.

Proof. Combine 5.14 and proof of 4.2. $\mathbf{m}_{5.19}$

Re mark 5.20. The parallels of 5.16-5.18 also hold. 
6. Variants of entangledness in ultraproducts. In this section we develop results of Section 1. The following improves 1.8:

Proposition 6.1. Assume that:

(a) $D$ is an ultrafilter on $\kappa$ and $E$ is an ultrafilter on $\theta$,

(b) $g_{\varepsilon}: \kappa \rightarrow \theta$ for $\varepsilon<\varepsilon(*)$ are such that if $\varepsilon_{1}<\varepsilon_{2}<\varepsilon(*)$ then $g_{\varepsilon_{1}} \neq g_{\varepsilon_{2}} \bmod D$, and if $\varepsilon<\varepsilon(*)$ and $A \in E$ then $g_{\varepsilon}^{-1}[A] \in D$,

(c) $\mathcal{I}$ is a linear order of the cardinality $\lambda \geq \theta$.

Then there exists a sequence $\left\langle f_{\varepsilon}^{\alpha} / D: \varepsilon<\varepsilon(*), \alpha<\lambda\right\rangle$ of pairwise distinct members of $\mathcal{I}^{\kappa} / D$ such that for each $\alpha<\beta<\lambda$, either

$$
(\forall \varepsilon<\varepsilon(*))\left(f_{\varepsilon}^{\alpha} / D<f_{\varepsilon}^{\beta} / D\right) \quad \text { or } \quad(\forall \varepsilon<\varepsilon(*))\left(f_{\varepsilon}^{\beta} / D<f_{\varepsilon}^{\alpha} / D\right) .
$$

In particular, the linear order $\mathcal{I}^{\kappa} / D$ is not entangled (here $\varepsilon(*)=2$ suffices; in fact, not $(\lambda, \varepsilon(*))$-entangled $)$ and the Boolean algebra $\left(\mathrm{BA}_{\mathrm{inter}}(\mathcal{I})\right)^{\kappa} / D$ is not $\lambda$-narrow.

Proof. Choose pairwise distinct $a_{\zeta}^{\alpha} \in \mathcal{I}$ for $\alpha<\lambda$ and $\zeta<\theta$. Let $f_{\varepsilon}^{\alpha}: \kappa \rightarrow \mathcal{I}$ be given by $f_{\varepsilon}^{\alpha}(i)=a_{g_{\varepsilon}(i)}^{\alpha}$. Note that if $\alpha_{1} \neq \alpha_{2}$ then $\{i<\kappa$ : $\left.f_{\varepsilon_{1}}^{\alpha_{1}}(i)=f_{\varepsilon_{2}}^{\alpha_{2}}(i)\right\}=\emptyset$. If $\alpha_{1}=\alpha_{2}=\alpha$ but $\varepsilon_{1} \neq \varepsilon_{2}$ then

$$
\left\{i \in \kappa: f_{\varepsilon_{1}}^{\alpha}(i)=f_{\varepsilon_{2}}^{\alpha}(i)\right\}=\left\{i \in \kappa: g_{\varepsilon_{1}}(i)=g_{\varepsilon_{2}}(i)\right\} \notin D
$$

(as $\left.g_{\varepsilon_{1}} \neq g_{\varepsilon_{2}} \bmod D\right)$. Consequently, if $\left(\alpha_{1}, \varepsilon_{1}\right) \neq\left(\alpha_{2}, \varepsilon_{2}\right)$ then $f_{\varepsilon_{1}}^{\alpha_{1}} / D \neq$ $f_{\varepsilon_{2}}^{\alpha_{2}} / D$. Suppose now that $\alpha<\beta<\lambda$. Let $A=\left\{\zeta<\theta: a_{\zeta}^{\alpha}<a_{\zeta}^{\beta}\right\}$. Assume that $A \in E$ and let $\varepsilon<\varepsilon(*)$. Then

$$
\left\{i<\kappa: f_{\varepsilon}^{\alpha}(i)<f_{\varepsilon}^{\beta}(i)\right\}=\left\{i<\kappa: a_{g_{\varepsilon}(i)}^{\alpha}<a_{g_{\varepsilon}(i)}^{\beta}\right\}=g_{\varepsilon}^{-1}[A] \in D
$$

and hence $f_{\varepsilon}^{\alpha} / D<f_{\varepsilon}^{\beta} / D$. Similarly, if $A \notin E$ then for each $\varepsilon<\varepsilon(*)$, $f_{\varepsilon}^{\alpha} / D>f_{\varepsilon}^{\beta} / D$.

Now clearly $\mathcal{I}^{\kappa} / D$ is not entangled, but what about the narrowness of $\left(\mathrm{BA}_{\text {inter }}(\mathcal{I})\right)^{\kappa} / D$ ? Recall that $\mathrm{BA}_{\text {inter }}\left(\mathcal{I}^{\kappa} / D\right)$ embeds in $\left(\mathrm{BA}_{\text {inter }}(\mathcal{I})\right)^{\kappa} / D$. So if the cardinality of $\mathrm{BA}_{\text {inter }}(\mathcal{I})$ is regular we can just quote $1.5(\mathrm{a}) \Leftrightarrow(\mathrm{c})$ (here $\sigma=\aleph_{0}$, see Definition 1.3). Otherwise, just note that for a linear order $\mathcal{J}$, if $a_{l}<b_{l}$ (for $l=0,1$ ) and $\left[a_{l}, b_{l}\right) \in \mathrm{BA}_{\text {inter }}(\mathcal{J})$ are comparable and $\left\{a_{0}, b_{0}, a_{1}, b_{1}\right\}$ is with no repetition then

$$
a_{0}<_{\mathcal{J}} a_{1} \Leftrightarrow \neg\left(b_{0}<_{\mathcal{J}} b_{1}\right) ;
$$

this can be applied by the statement above.

R e m a r k 6.2. Proposition 6.1 shows that entangledness can be destroyed by ultraproducts. Of course, to make this complete we have to say how one can get $D, E, g_{\varepsilon}$ 's satisfying (a)-(b) of 6.1 . But this is easy:

(1) For example, suppose that $E$ is a uniform ultrafilter on $\theta, D=E \times E$ is the product ultrafilter on $\theta \times \theta=\kappa, \varepsilon(*)=2$ and $g_{\varepsilon}: \theta \times \theta \rightarrow \theta$ (for 
$\varepsilon<2)$ are given by $g_{0}(i, j)=i$ and $g_{1}(i, j)=j$. Then $E, D, \varepsilon(*)$ and $g_{\varepsilon}$ satisfy the requirements (a)-(b) of 6.1 .

(2) More generally, assume that $\theta \leq \kappa, E$ is a non-principal ultrafilter on $\theta, \varepsilon(*) \leq 2^{\kappa}$. Let $g_{\varepsilon}: \kappa \rightarrow \theta$ for $\varepsilon<\varepsilon(*)$ constitute an independent family of functions. Then the family $\left\{g_{\varepsilon}^{-1}[A]: \varepsilon<\varepsilon(*), A \in E\right\}$ has the finite intersection property so we can complete it to an ultrafilter $D$. One can easily check that $D, E$ and $g_{\varepsilon}$ 's satisfy (a)-(b) of 6.1 .

Re mark 6.3. (1) In 6.1 we did not use " $<$ is a linear order". Thus for any binary relation $R$ the parallel (with $R, \neg R$ instead of $<,>$ ) holds. In particular, we can apply this to Boolean algebras.

(2) We can weaken assumption (b) in 6.1 and accordingly the conclusion. For example, we can replace (b) by

(b) ${ }^{*} \mathcal{P} \subseteq \mathcal{P}(\varepsilon(*))$ and for each $A \in E$,

$$
\left\{\varepsilon<\varepsilon(*): g_{\varepsilon}^{-1}[A] \in D\right\} \in \mathcal{P},
$$

and the conclusion by

$$
\left\{\varepsilon<\varepsilon(*): f_{\varepsilon}^{\alpha} / D<f_{\varepsilon}^{\beta} / D\right\} \in \mathcal{P} .
$$

(3) A kind of entangledness can be preserved by ultraproducts (see 6.4 below). More entangledness is preserved if we put additional demands on the ultrafilter (see 6.8).

(4) Let us explain why we introduced "positive entangledness" in 1.10. The proof of 6.1 excludes not only "full" entangledness but many variants (for the ultrapower). Now the positive $\sigma$-entangledness seems to be the maximal one not excluded. Rightly so by 6.4 .

(5) So if we can find a linear order $\mathcal{I}$ which is $\mu^{+}$-entangled for some $\mu$ such that $\mu^{\aleph_{0}}=\mu$ (or at least $\mu^{\aleph_{0}}<|\mathcal{I}|$ ) then we can answer Monk's problem from the introduction: if $D$ is a non-principal non-separative ultrafilter on $\omega$ (see Definition 6.5; they exist by $6.2(1)$ ), then $\mathcal{I}^{\omega} / D$ is not $\mu^{+}$-entangled (by 6.1). Thus if $B$ is the interval Boolean algebra of $\mathcal{I}$ then

$$
\operatorname{inc}(B) \leq \mu, \quad \mu^{\aleph_{0}}<|\mathcal{I}|, \quad \text { but } \quad \operatorname{inc}\left(B^{\omega} / D\right) \geq|\mathcal{I}|^{\aleph_{0}}
$$

(in fact, inc ${ }^{+}\left(B^{\omega} / D\right)=\left|\mathcal{I}^{\omega} / D\right|^{+}=\left(|\mathcal{I}|^{\omega} / D\right)^{+}$and inc $\left.{ }^{+}(B) \leq \mu^{+}\right)$. In fact, for any infinite Boolean algebra $B$ and a non-principal ultrafilter $D$ on $\omega$ we have $\operatorname{inc}\left(B^{\omega} / D\right) \geq(\operatorname{inc}(B))^{\omega} / D$ (as for $\lambda_{n}$ inaccessible the linear order $\prod_{n<\omega}\left(\lambda_{n},<\right) / D$ cannot be $\mu^{+}$-like (see [MgSh 433]).

Proposition 6.4. Suppose that $\kappa<\sigma<\lambda$ are regular cardinals such that $(\forall \theta<\lambda)\left(\theta^{<\sigma}<\lambda\right)$. Assume that $D$ is an ultrafilter on $\kappa$. If $\mathcal{I}$ is a positively $\sigma$-entangled linear order of size $\lambda$ then $\mathcal{I}^{\kappa} / D$ is positively $\sigma$-entangled. 
Proof. Suppose $f_{\varepsilon}^{\alpha} / D \in \mathcal{I}^{\kappa} / D$ (for $\varepsilon<\varepsilon(*)<\sigma$ and $\alpha<\lambda$ ) are such that

$$
(\forall \alpha<\beta<\lambda)(\forall \varepsilon<\varepsilon(*))\left(f_{\varepsilon}^{\alpha} / D \neq f_{\varepsilon}^{\beta} / D\right) .
$$

Let $u \in\{\emptyset, \varepsilon(*)\}$. For $\alpha<\lambda$ let $A_{\alpha}=\left\{f_{\varepsilon}^{\alpha}(i): \varepsilon<\varepsilon(*), i<\kappa\right\}, A_{<\alpha}=$ $\bigcup_{\beta<\alpha} A_{\beta}$ and $B_{\alpha}=A_{\alpha} \cap A_{<\alpha}$. Note that $\left|A_{\alpha}\right|<\sigma$ and $\left|A_{<\alpha}\right|<\lambda$ (for $\alpha<\lambda)$. If $\delta<\lambda$ and $\operatorname{cf}(\delta)=\sigma$ then $\left|B_{\delta}\right|<\operatorname{cf}(\delta)$ and consequently for some $h(\delta)<\delta$ we have $B_{\delta} \subseteq A_{<h(\delta)}$. By Fodor's lemma we find $\beta_{0}<\lambda$ and a stationary set $S \subseteq \lambda$ such that

$$
\delta \in S \Rightarrow \operatorname{cf}(\delta)=\sigma \& B_{\delta} \subseteq A_{<\beta_{0}} .
$$

For $\delta \in S$ let $Y_{\delta}=\left\{\left(\varepsilon, i, f_{\varepsilon}^{\delta}(i)\right): \varepsilon<\varepsilon(*), i<\kappa, f_{\varepsilon}^{\delta}(i) \in A_{<\beta_{0}}\right\}$. As there are $\left|A_{<\beta_{0}}\right|<\sigma<\lambda$ possibilities for $Y_{\delta}$, we can find a stationary set $S_{1} \subseteq S$ and $Y$ such that for $\delta \in S_{1}$ we have $Y_{\delta}=Y$. Let $\alpha, \beta \in S_{1}, \alpha<\beta$ and $\varepsilon<\varepsilon(*)$. If there is $x$ such that $(\varepsilon, i, x) \in Y$ then $f_{\varepsilon}^{\alpha}(i)=f_{\varepsilon}^{\beta}(i)$. Thus

$$
E_{\varepsilon}=\{i<\kappa:(\exists x)((\varepsilon, i, x) \in Y)\}=\emptyset \bmod D,
$$

as $\left\{i<\kappa: f_{\varepsilon}^{\alpha}(i)=f_{\varepsilon}^{\beta}(i)\right\}=\emptyset \bmod D$ (for distinct $\alpha, \beta \in S_{1}$ ). Clearly if $\alpha, \beta \in S_{1}, \alpha<\beta, \varepsilon<\varepsilon(*)$ and $i \in \kappa \backslash E_{\varepsilon}$ then $f_{\varepsilon}^{\alpha}(i) \neq f_{\varepsilon}^{\beta}(i)$. Hence we may apply the positive $\sigma$-entangledness of $\mathcal{I}$ to

$\left\{f_{\varepsilon}^{\alpha}(i): \alpha \in S_{1}, \varepsilon<\varepsilon(*), i \in \kappa \backslash E_{\varepsilon}\right\} \quad$ and $\quad u^{\prime}=\left\{(\varepsilon, i): \varepsilon \in u, i \in \kappa \backslash E_{\varepsilon}\right\}$.

Consequently, we have $\alpha<\beta$, both in $S_{1}$ and such that

$$
(\forall \varepsilon<\varepsilon(*))\left(\forall i \in \kappa \backslash E_{\varepsilon}\right)\left(f_{\varepsilon}^{\alpha}(i)<f_{\varepsilon}^{\beta}(i) \Leftrightarrow \varepsilon \in u\right) .
$$

Since $\kappa \backslash E_{\varepsilon} \in D$ we get $(\forall \varepsilon<\varepsilon(*))\left(f_{\varepsilon}^{\alpha} / D<f_{\varepsilon}^{\beta} / D \Leftrightarrow \varepsilon \in u\right)$. 6.4

Definition 6.5. An ultrafilter $D$ on $\kappa$ is called separative if for every $\bar{\alpha}, \bar{\beta} \in{ }^{\kappa} \kappa$ such that $(\forall i<\kappa)\left(\alpha_{i} \neq \beta_{i}\right)$ there is $A \in D$ such that

$$
\left\{\alpha_{i}: i \in A\right\} \cap\left\{\beta_{i}: i \in A\right\}=\emptyset .
$$

Remark 6.6. So 6.2(1) says that $D \times D$ is not separative.

Proposition 6.7. Suppose that $D$ is a separative ultrafilter on $\kappa, n<\omega$ and $\bar{\alpha}^{l} \in{ }^{\kappa} \kappa($ for $l<n)$ are such that

$$
\left(\forall l_{0}<l_{1}<n\right)\left(\bar{\alpha}^{l_{0}} / D \neq \bar{\alpha}^{l_{1}} / D\right) .
$$

Then there is $A \in D$ such that the sets $\left\{\alpha_{i}^{l}: i \in A\right\}($ for $l<n)$ are pairwise disjoint.

Proof. For $l<m<n$, by 6.5 , there is $A_{l, m} \in D$ such that

$$
\left\{\alpha_{i}^{l}: i \in A_{l, m}\right\} \cap\left\{\alpha_{i}^{m}: i \in A_{l, m}\right\}=\emptyset .
$$

Now $A=\bigcap_{l<m<n} A_{l, m}$ is as required. - 6.7 
Proposition 6.8. Assume $\mu=\operatorname{cf}(\mu)$ and $(\forall \alpha<\mu)\left(|\alpha|^{\kappa}<\mu\right)$. Suppose that $\mathcal{I}$ is a $\left(\mu, \kappa^{+}\right)$-entangled linear order and $D$ is a separative ultrafilter on $\kappa$. Then the linear order $\mathcal{I}^{\kappa} / D$ is $\left(\mu, \aleph_{0}\right)$-entangled.

Proof. Let $n<\omega$ and $u \subseteq n$, and let $f_{\alpha}^{l} / D \in \mathcal{I}^{\kappa} / D$ for $l<n$ and $\alpha<\mu$ be pairwise distinct. By 6.7 we may assume that (for each $\alpha<\mu$ ) the sets $\left\langle\left\{f_{\alpha}^{l}(i): i<\kappa\right\}: l<n\right\rangle$ are pairwise disjoint. Applying the $\Delta$-lemma we may assume that $\left\{\left\langle f_{\alpha}^{l}(i): i<\kappa, l<n\right\rangle: \alpha<\mu\right\}$ forms a $\Delta$-system of sequences and that the diagram of the equalities does not depend on $\alpha$. Let

$$
A=\left\{(i, l) \in \kappa \times n:(\forall \alpha<\beta<\mu)\left(f_{\alpha}^{l}(i)=f_{\beta}^{l}(i)\right)\right\}
$$

(i.e. the heart of the $\Delta$-system). Note that for each $l<n$ the set $\{i \in \kappa$ : $(i, l) \in A\}$ is not in $D$ (as $f_{\alpha}^{l} / D$ 's are pairwise distinct). Consequently, we may modify the functions $f_{\alpha}^{l}$ and we may assume that $A=\emptyset$. Now we have

$$
f_{\alpha_{0}}^{l_{0}}\left(i_{0}\right)=f_{\alpha_{1}}^{l_{1}}\left(i_{1}\right) \Rightarrow \alpha_{0}=\alpha_{1} \& l_{0}=l_{1} .
$$

It is now easy to apply the $\left(\mu, \kappa^{+}\right)$-entangledness of $\mathcal{I}$ and find $\alpha<\beta<\mu$ such that $f_{\alpha}^{l} / D<f_{\beta}^{l} / D \equiv l \in u$. 6.8

Proposition 6.9. (1) If $D$ is a selective ultrafilter on $\kappa$ (i.e. for every $f: \kappa \rightarrow \kappa$ there is $A \in D$ such that either $f\lceil A$ is constant or $f \uparrow A$ is one-to-one) then $D$ is separative.

(2) If no uniform ultrafilter on $\omega$ is generated by less than continuum sets $\left(\right.$ i.e. $\mathfrak{u}=2^{\aleph_{0}}$ ) then there exists a separative ultrafilter on $\omega$.

Proof. (1) Suppose that $\bar{\alpha}, \bar{\beta} \in{ }^{\kappa} \kappa$ are such that $(\forall i<\kappa)\left(\alpha_{i} \neq \beta_{i}\right)$. We find $A \in D$ such that $\bar{\alpha}\lceil A$ and $\bar{\beta}\lceil A$ are either constant or one-to-one. If at least one of them is constant then, possibly omitting one element from $A$, the sets $\left\{\alpha_{i}: i \in A\right\}$ and $\left\{\beta_{i}: i \in A\right\}$ are disjoint, so assume that both sequences are one-to-one. Choose inductively $m_{i} \in\{0,1,2\}$ (for $i \in A$ ) such that

$$
i, j \in A \& \alpha_{i}=\beta_{j} \Rightarrow m_{i} \neq m_{j} .
$$

There are $m^{*}$ and $B \subseteq A, B \in D$ such that $(\forall i \in B)\left(m_{i}=m^{*}\right)$. Then

$$
\left\{\alpha_{i}: i \in B\right\} \cap\left\{\beta_{i}: i \in B\right\}=\emptyset .
$$

(2) Straightforward. $\mathbf{\square}_{6.9}$

Proposition 6.10. If $D$ is a uniform not separative ultrafilter on $\kappa$ and $\mathcal{I}$ is a linear order of size $\lambda \geq \kappa$ then the linear order $\mathcal{I}^{\kappa} / D$ is not $(\lambda, 2)$ entangled.

Proof. As $D$ is not separative we have $\bar{\alpha}, \bar{\beta} \in{ }^{\kappa} \kappa$ witnessing it. This means that $\bar{\alpha} \neq \bar{\beta} \bmod D$ and the family

$$
\left\{\left\{\alpha_{i}: i \in A\right\},\left\{\beta_{i}: i \in A\right\}: A \in D\right\}
$$


has the finite intersection property, so can be extended to an ultrafilter $E$ on $\kappa$. Let $\varepsilon(*)=2, \theta=\kappa, g_{0}(i)=\alpha_{i}, g_{1}(i)=\beta_{i}$. Consequently, we may apply 6.1. 6.10

Conclusion 6.11. (1) If $\theta^{\kappa} / D>2^{2^{\theta}}$ then $D$ is not separative.

(2) If $D$ is a regular ultrafilter on $\kappa$ and $2^{\kappa}>\beth_{2}$ then $D$ is not separative.

$\mathrm{R}$ e m ark 6.12. If there is no inner model with measurable cardinals then every $D$ is regular or close enough to this to give the result.

Proof of Conclusions 6.11. (1) For every $f \in{ }^{\kappa} \theta$ the family $E_{f}=\left\{A \subseteq \theta: f^{-1}[A] \in D\right\}$ is an ultrafilter on $\theta$. For some $g_{0}, g_{1} \in{ }^{\kappa} \theta$ we have $E_{g_{0}}=E_{g_{1}}$ but $g_{0} / D \neq g_{1} / D$ and hence we are done.

(2) The regularity implies $\aleph_{0}^{\kappa} / D=2^{\kappa}$ (see [CK]), so by the first part we are done. $\mathbf{-}_{6.11}$

DeFinition 6.13. Let $\kappa, \sigma$ be cardinal numbers.

(1) We say that a linear order $\mathcal{I}$ is strongly $(\mu, \sigma)$-entangled if: $(|\mathcal{I}|, \mu \geq$ $\sigma+\aleph_{0}$ and) for every $\varepsilon(*)<1+\sigma, t_{\alpha}^{\zeta} \in \mathcal{I}$ (for $\left.\alpha<\mu, \zeta<\varepsilon(*)\right)$ and $u \subseteq \varepsilon(*)$ such that

$$
\alpha<\mu \& \zeta \in u \& \xi \in \varepsilon(*) \backslash u \Rightarrow t_{\alpha}^{\zeta} \neq t_{\alpha}^{\xi}
$$

for some $\alpha<\beta<\mu$ we have:

$$
\begin{aligned}
& \text { (a) } \varepsilon \in u \Rightarrow t_{\alpha}^{\varepsilon} \leq_{\mathcal{I}} t_{\beta}^{\varepsilon}, \\
& \text { (b) } \varepsilon \in \varepsilon(*) \backslash u \Rightarrow t_{\beta}^{\varepsilon} \leq_{\mathcal{I}} t_{\alpha}^{\varepsilon} .
\end{aligned}
$$

(2) We say that a linear order $\mathcal{I}$ is strongly positively $[$ positively*] $(\mu, \sigma)$ entangled if for every $\varepsilon(*)<1+\sigma, t_{\alpha}^{\zeta} \in \mathcal{I}$ (for $\alpha<\mu, \zeta<\varepsilon(*)$ ) and $u \in\{\emptyset, \varepsilon(*)\}$, for some $\alpha<\beta<\mu$ [for some $\alpha \neq \beta<\mu$ ] we have
(a) $\varepsilon \in u \Rightarrow t_{\alpha}^{\varepsilon} \leq_{\mathcal{I}} t_{\beta}^{\varepsilon}$,
(b) $\varepsilon \in \varepsilon(*) \backslash u \Rightarrow t_{\beta}^{\varepsilon} \leq_{\mathcal{I}} t_{\alpha}^{\varepsilon}$.

Remark 6.14. For "positively*" it is enough to use $u=\varepsilon(*)$, so only clause (a) applies. [Why? as we can interchange $\alpha, \beta$.]

Proposition 6.15. (1) If $\sigma=\aleph_{0}$ and $\mu=\operatorname{cf}(\mu) \geq \sigma$ then in Definition 6.13(1) we can weaken $\alpha<\beta<\mu$ to $\alpha \neq \beta(<\mu)$. [Why? As in 1.2(6).]

(2) For a linear order, "strongly $(\mu, \sigma)$-entangled" implies both " $(\mu, \sigma)$ entangled" and "strongly positively $(\mu, \sigma)$-entangled" and the last implies "positively $(\mu, \sigma)$-entangled". Lastly, "strongly positively $(\mu, \sigma)$-entangled" implies "strongly positively* $(\mu, \sigma)$-entangled".

(3) In Definition 6.13 the properties are preserved when increasing $\mu$ and/or decreasing $\sigma$ and/or decreasing $\mathcal{I}$.

(4) If $\mu=\operatorname{cf}(\mu)$ and $(\forall \varepsilon<\sigma)(\forall \theta<\mu)\left(\theta^{|\varepsilon|}<\mu\right)$ then:

(a) $\mathcal{I}$ is $(\mu, \sigma)$-entangled if and only if $\mathcal{I}$ is strongly $(\mu, \sigma)$-entangled, 
(b) $\mathcal{I}$ is positively $(\mu, \sigma)$-entangled if and only if $\mathcal{I}$ is strongly positively $(\mu, \sigma)$-entangled.

(5) If $\mu=\operatorname{cf}(\mu)$ and $(\forall \varepsilon<\sigma)\left(2^{|\varepsilon|}<\mu\right)$ then in Definition 6.13(1), (2) we can assume

$$
(\forall \alpha<\mu)(\forall \zeta<\xi<\varepsilon(*))\left(t_{\alpha}^{\zeta} \neq t_{\alpha}^{\xi}\right) .
$$

(6) $(\mu,<)$ and $(\mu,>)$ are not strongly $(\mu, 2)$-entangled (even if $\mathcal{I}$ is strongly $(\mu, 2)$-entangled then there is no partial function $f$ from $\mathcal{I}$ to $\mathcal{I}$ such that $x<_{\mathcal{I}} f(x),|\operatorname{dom}(f)|=\mu$ and $f$ preserves $\left.<_{\mathcal{I}}\right)$.

(7) If $(\forall \varepsilon<\sigma)\left(|\mathcal{I}|^{|\varepsilon|}<\mu\right)$ then $\mathcal{I}$ is strongly $(\mu, \sigma)$-entangled.

(8) Assume $\sigma$ is a limit cardinal and $\mathcal{I}$ is a linear order. Then:

(a) $\mathcal{I}$ is strongly $(\mu, \sigma)$-entangled if and only if for every $\sigma_{1}<\sigma$ the order $\mathcal{I}$ is strongly $\left(\mu, \sigma_{1}\right)$-entangled.

(b) Similarly for other notions of 1.1, 1.10, 6.13. 6.15

Proposition 6.16. Assume that $\mathcal{I}$ is a $(\mu, 2)$-entangled linear order and $\theta=\operatorname{cf}(\theta)<\mu$. Then for some $A \subseteq \mathcal{I}$ with $|A|<\mu$ we have

$$
x<_{\mathcal{I}} y \&\{x, y\} \nsubseteq A \Rightarrow\left|(x, y)_{\mathcal{I}}\right|>\theta .
$$

Proof. Let $E_{\mathcal{I}}^{\theta}$ be the following binary relation on $\mathcal{I}: x E_{\mathcal{I}}^{\theta} y$ if and only if

$$
x=y \quad \text { or } \quad\left[x<_{\mathcal{I}} y \&\left|(x, y)_{\mathcal{I}}\right|<\theta\right] \quad \text { or } \quad\left[y<_{\mathcal{I}} x \&\left|(y, x)_{\mathcal{I}}\right|<\theta\right] .
$$

Obviously:

(a) $E_{\mathcal{I}}^{\theta}$ is an equivalence relation,

(b) each equivalence class has cardinality $\leq \theta$,

(c) if an equivalence class has cardinality $\theta$ then there is a monotonic sequence of length $\theta$ in it, so necessarily $\theta^{+}<\mu$.

It is enough to show that the set $A=\left\{x:\left|x / E_{\mathcal{I}}^{\theta}\right|>1\right\}$ has cardinality less than $\mu$. Suppose that $|A| \geq \mu$. Then there are at least $\mu$ equivalence classes (as each class is of size $\leq \theta<\mu$ or of size $\leq \theta^{+}<\mu$ ). Consequently, we can find $t_{\alpha}^{0}, t_{\alpha}^{1} \in \mathcal{I}$ for $\alpha<\mu$ such that $t_{\alpha}^{0}<t_{\alpha}^{1}, t_{\alpha}^{0} E_{\mathcal{I}}^{\theta} t_{\alpha}^{1}$ and there is no repetition in $\left\{t_{\alpha}^{0} / E_{\mathcal{I}}^{\theta}: \alpha<\mu\right\}$. For $\varepsilon(*)=2$ and $u=\{0\}$ we get a contradiction with Definition 6.13.

R e m ark. Clearly, $\theta=\operatorname{cf}(\theta)$ is redundant.

Proposition 6.17. If $\mathcal{I}$ is strongly $(\mu, \sigma)$-entangled, $\aleph_{0} \leq \theta<\sigma$ and $\mu \leq|\mathcal{I}|$ then:

(a) $2^{\theta}<\mu$

(b) the cardinal $\chi:=\mid\left\{x / E_{\mathcal{I}}^{\theta}: x / E_{\mathcal{I}}^{\theta}\right.$ not a singleton $\} \mid$ satisfies $\chi^{\theta}<\mu$ (where $E_{\mathcal{I}}^{\theta}$ is from the proof of 6.16 ). 
Proof. Take $x_{0}^{0}<_{\mathcal{I}} x_{0}^{1}<_{\mathcal{I}} x_{1}^{0}<_{\mathcal{I}} x_{1}^{1}$. For $f \in{ }^{\theta} 2$ let $t_{f}^{2 \varepsilon+l}=x_{f(\varepsilon)}^{l}$ (for $\varepsilon<\theta$ ). If $2^{\theta} \geq \mu$ then we may consider $u=\{2 \varepsilon: \varepsilon<\theta\}$ and find (by the strong $(\mu, \sigma)$-entangledness) functions $f \neq g$ such that for all $\varepsilon<\theta$,

$$
t_{f}^{2 \varepsilon} \leq_{\mathcal{I}} t_{g}^{2 \varepsilon}, \quad t_{f}^{2 \varepsilon+1} \geq_{\mathcal{I}} t_{g}^{2 \varepsilon+1} .
$$

But if $\varepsilon<\theta$ is such that $f(\varepsilon) \neq g(\varepsilon)$ then we get $x_{f(\varepsilon)}^{0}<_{\mathcal{I}} x_{g(\varepsilon)}^{0}$. Hence $f(\varepsilon)=0, g(\varepsilon)=1$ and consequently $x_{f(\varepsilon)}^{1}<_{\mathcal{I}} x_{g(\varepsilon)}^{1}$. But the last contradicts $t_{f}^{2 \varepsilon+1} \geq_{\mathcal{I}} t_{g}^{2 \varepsilon+1}$. Hence $2^{\theta}<\mu$ as required in (a).

For (b), let $\left\langle x_{i}^{0} / E_{\mathcal{I}}^{\theta}: i<\chi\right\rangle$ be with no repetition, $x_{i}^{1} \in x_{i}^{0} / E_{\mathcal{I}}^{\theta}$ and $x_{i}^{0}<_{\mathcal{I}} x_{i}^{1}$. Let $\left\{f_{\alpha}: \alpha<\chi^{\theta}\right\}$ list all functions $f: \theta \rightarrow \chi$ (so for each $\alpha<\beta$ there is $\varepsilon<\theta$ such that $f_{\alpha}(\varepsilon) \neq f_{\beta}(\varepsilon)$ ). Let $t_{\alpha}^{2 \varepsilon+l}$ (for $\alpha<\lambda, \varepsilon<\theta$ and $l<2)$ be $x_{f_{\alpha}(\varepsilon)}^{l}$ and $u=\{2 \varepsilon: \varepsilon<\theta\} \subseteq \theta$ (so $\varepsilon(*)=\theta$ ). If $\mu \leq \chi^{\theta}$ then we get $\alpha<\beta<\mu$ by Definition 6.13(1) and we get a contradiction, so $\mu>\chi^{\theta}$ as required. $\mathbf{\square}_{6.17}$

Rem ark. See more in Shafir-Shelah [SaSh 553].

Proposition 6.18. If $\mathcal{I}$ is a linear order of density $\leq \theta$ and $|\mathcal{I}| \geq \theta \in$ $\left[\aleph_{0}, \sigma\right)$ then $\mathrm{BA}_{\text {inter }}^{\sigma}(\mathcal{I})$ is not $2^{\theta}$-narrow.

Pr o of. Choose $\mathcal{J} \subseteq \mathcal{I}$ with $|\mathcal{J}|=\theta$ such that for every $t \in \mathcal{J}$ for some $\tau \in \mathrm{BA}_{\text {inter }}^{\sigma}(\mathcal{I})$ we have $\tau \cap \mathcal{J}=\{t\}\left(\tau=\bigcap\left\{[t, s): t<_{\mathcal{I}} s \in \mathcal{J}\right\}\right)$. Hence for every $\mathcal{J}^{\prime} \subseteq \mathcal{J}$ for some $\tau \in \mathrm{BA}_{\text {inter }}^{\sigma}(\mathcal{I})$ we have $\tau \cap \mathcal{J}=\mathcal{J}^{\prime}$. The conclusion now follows. 6.18

Proposition 6.19. In Definition 6.13(1), if $\mathrm{cf}(\mu)=\mu$ (or less) we can without loss of generality demand

$$
(\forall \alpha<\mu)(\forall \zeta<\xi<\varepsilon(*))\left(t_{\alpha}^{\zeta} \neq t_{\alpha}^{\xi}\right)
$$

and for some linear order $<^{*}$ on $\varepsilon(*)$,

$$
\zeta<^{*} \xi, \zeta, \xi<\varepsilon(*) \Rightarrow t_{\alpha}^{\zeta}<_{\mathcal{I}} t_{\alpha}^{\xi} .
$$

Proof. Clearly the new version of the definition implies the old one. So now assume the old definition and we shall prove the new one. Let $\varepsilon(*)<1+\sigma$ and $t_{\alpha}^{\zeta}$ (for $\alpha<\mu, \zeta<\varepsilon(*)$ ). By 6.17(a) we have $\mu>2^{|\varepsilon(*)|}$ so we can replace $\left\langle\left\langle t_{\alpha}^{\zeta}: \zeta<\varepsilon(*)\right\rangle: \alpha<\mu\right\rangle$ by $\left\langle\left\langle t_{\alpha}^{\zeta}: \zeta<\varepsilon(*)\right\rangle: \alpha \in A\right\rangle$ for a suitable $A \in[\mu]^{\mu}$, and we are done. $\mathbf{\square}_{6.19}$

Proposition 6.20. (1) Assume $\sigma \geq \aleph_{0}$ and $\operatorname{cf}(\mu)=\mu$. Then in Definition 6.13(1), if we allow first to discard $<\mu$ members of $\mathcal{I}$ we can add

(c) for $\zeta, \xi<\varepsilon(*)$, if $t_{\alpha}^{\zeta}<_{\mathcal{I}} t_{\alpha}^{\xi}$ then $\left\{t_{\alpha}^{\zeta}, t_{\beta}^{\zeta}\right\}<_{\mathcal{I}}\left\{t_{\alpha}^{\xi}, t_{\beta}^{\xi}\right\}$ (i.e. we get an equivalent definition).

(2) Even without the "if we allow first to discard $<\mu$ members of $\mathcal{I}$ " part (1) still holds true. It also holds for $(\mu, \sigma)$-entangledness. 
Proof. (1) The new definition is apparently stronger so we have to prove that it follows from the old one. We can assume $\mu>\aleph_{0}$. By 6.16, without loss of generality,

$$
x<_{\mathcal{I}} y \Rightarrow\left|(x, y)_{\mathcal{I}}\right|>2|\varepsilon(*)|^{2} .
$$

Let $\varepsilon(*)<\sigma$ and $t_{\alpha}^{\zeta} \in \mathcal{I}$ be given. By 6.19 we may assume that

$$
(\forall \alpha<\mu)(\forall \zeta<\xi<\varepsilon(*))\left[t_{\alpha}^{\zeta} \neq t_{\alpha}^{\xi} \&\left(t_{\alpha}^{\zeta}<t_{\alpha}^{\xi} \equiv \zeta<^{*} \xi\right)\right] .
$$

So for $\alpha<\mu$ and $\zeta<^{*} \xi$ we can choose $s_{\alpha}^{\zeta, \xi, l} \in \mathcal{I}(l=1,2)$ such that for each $\alpha<\mu$,

$$
t_{\alpha}^{\zeta}<_{\mathcal{I}} s_{\alpha}^{\zeta, \xi, 1}<_{\mathcal{I}} s_{\alpha}^{\zeta, \xi, 2}<_{\mathcal{I}} t_{\alpha}^{\xi}
$$

and there are no repetitions in $\left\{t_{\alpha}^{\zeta}, s_{\alpha}^{\zeta, \xi, l}: \zeta<\varepsilon(*), \zeta<^{*} \xi\right.$ and $\left.l \in\{1,2\}\right\}$. So for some $\alpha<\beta$ we have

$$
\begin{array}{ll}
\zeta \in u & \Rightarrow t_{\alpha}^{\zeta} \leq_{\mathcal{I}} t_{\beta}^{\zeta}, \\
\zeta \in \varepsilon(*) \backslash u & \Rightarrow t_{\alpha}^{\zeta} \geq_{\mathcal{I}} t_{\beta}^{\zeta}, \\
\zeta<^{*} \xi & \Rightarrow s_{\alpha}^{\zeta, \xi, 1} \leq_{\mathcal{I}} s_{\beta}^{\zeta, \xi, 1}, \\
\zeta<^{*} \xi & \Rightarrow s_{\alpha}^{\zeta, \xi, 2} \geq_{\mathcal{I}} s_{\beta}^{\zeta, \xi, 2} .
\end{array}
$$

Now (c) follows immediately.

(2) Use $6.16+6.17(\mathrm{~b})$. The $(\mu, \sigma)$-entangledness is straightforward. 6.20

Proposition 6.21. Assume $\aleph_{0} \leq \theta<\sigma, \chi=\operatorname{dens}(\mathcal{I})$ and: $\mathcal{I}$ is strongly $(\mu, \sigma)$-entangled or $\mathrm{BA}_{\text {inter }}^{\sigma}(\mathcal{I})$ is $\mu$-narrow. Then $\chi^{\theta}<\mu$.

Proof. First note the following fact:

Claim 6.21.1. Assume that $\left\langle\mathcal{I}_{\varepsilon}: \varepsilon<\theta\right\rangle$ is a sequence of pairwise disjoint convex subsets of $\mathcal{I}, \chi_{\varepsilon}=\operatorname{dens}\left(\mathcal{I}_{\varepsilon}\right) \geq \aleph_{0}$ and $\chi=\prod_{\varepsilon<\theta} \chi_{\varepsilon}$. Then $\chi<\mu$ and $\mathrm{BA}_{\text {inter }}^{\sigma}(\mathcal{I})$ is not $\chi$-narrow.

Proof. By induction on $i<\chi_{\varepsilon}$, choose $a_{i}^{\varepsilon}<b_{i}^{\varepsilon}$ from $\mathcal{I}_{\varepsilon}$ such that $\left[a_{i}^{\varepsilon}, b_{i}^{\varepsilon}\right]_{\mathcal{I}}$ is disjoint from $\left\{a_{j}^{\varepsilon}, b_{j}^{\varepsilon}: j<i\right\}$. Let $\left\{f_{\alpha}: \alpha<\chi\right\}$ list $\prod_{\varepsilon<\theta} \chi_{\varepsilon}$ (with no repetitions), let $\varepsilon(*)=\theta$, and let $t_{\alpha}^{2 \varepsilon+l}$ be $a_{f_{\alpha}(\varepsilon)}^{\varepsilon}$ if $l=0, b_{f_{\alpha}(\varepsilon)}^{\varepsilon}$ if $l=1$. For $u=\{2 \varepsilon: \varepsilon<\theta\}$ we get a contradiction with " $\mathcal{I}$ is strongly $(\chi, \sigma)$-entangled". The proof for the Boolean version is similar. 6.21 .1

By an argument similar to that of 6.21 .1 one can show that $\chi(=\operatorname{dens}(\mathcal{I}))$ $<\mu$. So the interesting case is when $\chi^{\theta}>\theta$. As $2^{\theta}<\mu$ (see 6.15) we may assume that $\chi>2^{\theta}$. Let $\chi_{1}=\min \left\{\lambda: \lambda^{\theta} \geq \chi\right\}$, so $\operatorname{cf}\left(\chi_{1}\right) \leq \theta$, and let $\chi_{1}=\sum_{\varepsilon<\theta(*)} \chi_{1, \varepsilon}^{+}, \theta(*)=\operatorname{cf}\left(\chi_{1}\right) \leq \theta$ and $\chi_{1, \varepsilon}^{\theta}<\chi_{1}$. For each $\varepsilon<\theta(*)$ we define a binary relation $E_{\varepsilon}^{*}$ on $\mathcal{I}$ :

$$
x E_{\varepsilon}^{*} y \quad \text { if and only if either } x=y \text { or } x<y, \operatorname{dens}\left(\mathcal{I}\lceil(x, y)) \leq \chi_{1, \varepsilon}\right. \text { or }
$$

$$
y<x, \operatorname{dens}(\mathcal{I} \uparrow(y, x)) \leq \chi_{1, \varepsilon} .
$$

It is an equivalence relation, each equivalence class has density $\leq \chi_{1, \varepsilon}^{+}$, and 
the number of $E_{\varepsilon}^{*}$-equivalence classes is $\geq \chi$. So by the Erdös-Rado theorem we can find a monotonic sequence $\left\langle x_{i}^{\varepsilon}: i<\theta^{+}\right\rangle$such that $i \neq j \Rightarrow$ $\neg\left(x_{i}^{\varepsilon} E_{\varepsilon}^{*} x_{j}^{\varepsilon}\right)$.

Without loss of generality, for all $\varepsilon$ the monotonicity is the same, so we can assume $i<j \Rightarrow x_{i}^{\varepsilon}<_{\mathcal{I}} x_{j}^{\varepsilon}$. Discarding a long initial segment from each $\left\langle x_{i}^{\varepsilon}: i<\theta^{+}\right\rangle$we may assume that for each $\varepsilon, \zeta<\theta(*)$ either

$$
\left(\forall i<\theta^{+}\right)\left(\exists j<\theta^{+}\right)\left(x_{i}^{\varepsilon}<_{\mathcal{I}} x_{j}^{\zeta} \& x_{i}^{\zeta}<_{\mathcal{I}} x_{j}^{\varepsilon}\right)
$$

or

$$
\bigcup_{i<j}\left[x_{i}^{\varepsilon}, x_{j}^{\varepsilon}\right]_{\mathcal{I}} \text { and } \bigcup_{i<j}\left[x_{i}^{\zeta}, x_{j}^{\zeta}\right]_{\mathcal{I}} \quad \text { are disjoint. }
$$

Now it is easy to satisfy the assumptions of 6.21.1. 6.21

Conclusion 6.22. Assume that $\mu=\operatorname{cf}(\mu) \geq \sigma=\operatorname{cf}(\sigma) \geq \aleph_{0}$ and $\mathcal{I}$ is a linear order of cardinality $\geq \mu$. Then in Definition 6.13(1) we can demand (*) of $1.2(3)$.

Proof. By 6.21 (and see the proof of 1.2(3)).

Proposition 6.23. Assume $\mu=\operatorname{cf}(\mu)>\sigma=\operatorname{cf}(\sigma) \geq \aleph_{0}$ and $\mathcal{I}$ is a linear order with $|\mathcal{I}| \geq \mu$. Then the following conditions are equivalent:

(a) $\mathcal{I}$ is strongly $(\mu, \sigma)$-entangled,

(b) $\mathrm{BA}_{\text {inter }}^{\sigma}(\mathcal{I})$ is $\mu$-narrow.

Proof. $(\mathrm{a}) \Rightarrow(\mathrm{b})$. By 6.22 the situation is similar enough to the one in 1.5 to carry out the proof as there.

(b) $\Rightarrow(\mathrm{a})$. By 6.21 we can apply the parallel of $1.2(3)$, so the situation is similar enough to the one in 1.5 to carry out the proof as there.

6.23

Proposition 6.24. Assume $\sigma=\theta^{+}>\aleph_{0}$ and $\mu>\sigma$.

(1) If $\mathcal{I}$ is not strongly [or strongly positively] [or strongly positively*] $\left(\mu_{i}, \sigma\right)$-entangled for $i<\theta$ then $\mathcal{I}$ is not strongly [or strongly positively] [or strongly positively $\left.{ }^{*}\right](\mu, \sigma)$-entangled for $\mu=\prod_{i<\theta} \mu_{i}$.

(2) If $\mu$ is singular and $\mathcal{I}$ is strongly $(\mu, \sigma)$-entangled then for some $\mu^{\prime}<\mu, \mathcal{I}$ is strongly $\left(\mu^{\prime}, \sigma\right)$-entangled (so this holds for every large enough $\left.\mu^{\prime}<\mu\right)$.

(3) The parallel of (2) holds for "strongly $(\mu, \sigma)$-entangled", "strongly positively $(\mu, \sigma)$-entangled" and "strongly positively* $(\mu, \sigma)$-entangled".

Proof. (1) First we deal with "strongly $(\mu, \sigma)$-entangled". We know $|\mathcal{I}| \geq \sigma$. Suppose that $\left\langle t_{\alpha}^{i, \zeta}: \alpha<\mu_{i}, \quad \zeta<\varepsilon_{i}(*)\right\rangle$ and $u_{i}$ form a counterexample for $\mu_{i}$. As we can extend the sequences and $u_{i}$, we can assume $\left|u_{i}\right|=\left|\varepsilon_{i}(*) \backslash u_{i}\right|=\theta$. So by renaming $\varepsilon_{i}(*)=\theta, u_{i}=\{2 \zeta: \zeta<\theta\}$. Let $f_{\beta} \in \prod_{i<\theta} \mu_{i}$ for $\beta<\mu$ be pairwise distinct. Choose $\varepsilon(*)=\theta \cdot \theta, t_{\beta}^{\theta i+\zeta}=t_{f_{\beta}(i)}^{i, \zeta}$ for $i<\theta, \zeta<\theta$ and $\beta<\mu$, and $u=\{2 \zeta: \zeta<\theta \cdot \theta\}$. Now check. 
For the cases "strongly positively*" $\left(\mu_{i}, \sigma\right)$-entangled, first, without loss of generality, $\varepsilon_{i}(*)=\theta$; then as $u_{i}$ has two values, for some $u^{*}$ we have

$$
\prod\left\{\mu_{i}: i<\theta, u_{i}=u^{*}\right\}=\mu .
$$

Thus without loss of generality $u_{i}=u$ and let $u=\bigcup_{i<\theta}\{i\} \times u^{*}$. Next proceed as above.

(2) Assume not. Let $\mu=\sum_{i<\kappa} \mu_{i}$ with $\kappa<\mu_{i}<\mu, \kappa=\operatorname{cf}(\mu)$ and $i<j \Rightarrow \mu_{i}<\mu_{j}$. So for each $i<\kappa$ we can find a sequence $\left\langle t_{\alpha}^{i, \zeta}: \zeta<\right.$ $\left.\varepsilon_{i}(*), \alpha<\mu_{i}^{+}\right\rangle, u_{i}$ exemplifying the failure of " $\mathcal{I}$ is $\left(\mu_{i}^{+}, \sigma\right)$-entangled". Thus for each $i$ and for every $\alpha<\mu_{i}^{+}$, there are no repetitions in $\left\{t_{\alpha}^{i, \zeta}: \zeta<\varepsilon_{i}(*)\right\}$ and $|\mathcal{I}| \geq \mu$. Now let $\varepsilon(*)=\theta+\theta$ and $u=\{2 \zeta: \zeta<\theta+\theta\}$. For $\zeta<\theta$ and $\beta \in \mu_{i}^{+} \backslash \bigcup\left\{\mu_{j}^{+}: 0<j<i\right\}$ we put $t_{\beta}^{\zeta}=t_{\beta}^{i, \zeta}, t_{\beta}^{\theta+\zeta}=t_{i}^{0, \zeta}$. Without loss of generality for every $\alpha<\mu$ there are no repetitions in $\left\{t_{\alpha}^{\zeta}: \zeta<\theta+\theta\right\}$. Now check.

(3) Let $\kappa, \mu_{i}$ (for $\left.i<\kappa\right), t_{\alpha}^{i, \zeta}$ (for $i<\kappa, \zeta<\varepsilon_{i}(*)$ and $\alpha<\mu_{i}^{*}$ ) and $u_{i}$ be as in the proof of (2) (for the appropriate notion). Again we can assume $\varepsilon_{i}(*)=\theta$ and $u_{i}=u^{*}$, but the choice of $\alpha_{i}$ does not transfer. But by $6.24(1)$ we have $\mu_{i}^{\theta}<\mu$ and hence without loss of generality $\left(\sum_{j<i} \mu_{j}^{+}\right)^{\theta} \leq \mu_{i}=\mu_{i}^{\theta}$, so for some $v_{i} \subseteq \varepsilon_{i}(*)$,

$$
\left(\zeta \in v_{i} \Rightarrow\left[t_{\alpha}^{i, \zeta}=t_{\beta}^{i, \zeta} \Leftrightarrow \alpha=\beta\right]\right) \quad \text { and } \quad\left[\zeta \in \varepsilon_{i}(*) \backslash v_{i} \Rightarrow t_{\alpha}^{i, \zeta}=t_{\beta}^{i, \zeta}\right]
$$

and $t_{\alpha}^{i, \zeta}=t_{\alpha}^{i, \xi} \Leftrightarrow t_{\beta}^{i, \zeta}=t_{\beta}^{i, \xi}$. We can omit $\varepsilon_{i}(*) \backslash v_{i}$ etc., so $\left\langle t_{\alpha}^{i, \zeta}: \zeta<\right.$ $\left.\varepsilon_{i}(*), \alpha<\mu_{i}^{+}\right\rangle$is with no repetition and proceed as there. $\mathbf{\square}_{6.24}$

Re m a rk 6.25. In 6.24(2) we cannot replace "strongly entangled" by "entangled"; see [SaSh 553].

Conclusion 6.26. If $|\mathcal{I}| \geq \mu>\sigma=\theta^{+}>\aleph_{0}$ then $\mathcal{I}$ is $(\mu, \sigma)$-entangled if and only if $\mathcal{I}$ is strongly $(\mu, \sigma)$-entangled.

Conclusion 6.27. If $\mu \geq \sigma=\theta^{+} \geq \aleph_{0}$ and $\mathcal{I}$ is a strongly $(\mu, \sigma)$ entangled linear order then for some regular $\mu^{*}$ we have $\mu^{*} \leq \mu,\left(\forall \alpha<\mu^{*}\right)$ $\left(|\alpha|^{\theta}<\mu^{*}\right)$ and $\mathcal{I}$ is (strongly) $\left(\mu^{*}, \sigma\right)$-entangled. Consequently, in 6.23 regularity is not needed.

\section{References}

[ARSh 153] U. Abraham, M. Rubin and S. Shelah, On the consistency of some partition theorems for continuous colorings, and the structure of $\aleph_{1}$-dense real order types, Ann. Pure Appl. Logic 29 (1985), 123-206.

[AbSh 106] U. Avraham [Abraham] and S. Shelah, Martin's axiom does not imply that every two $\aleph_{1}$-dense sets of reals are isomorphic, Israel J. Math. 38 (1981), 161-176. 
[Bo] R. Bonnet, Sur les algèbres de Boole rigides, $\mathrm{PhD}$ thesis, Université Lyon 1, 1978.

[BoSh 210] R. Bonnet and S. Shelah, Narrow Boolean algebras, Ann. Pure Appl. Logic 28 (1985), 1-12.

[CK] C. C. Chang and H. J. Keisler, Model Theory, Stud. Logic Found. Math. 73, North-Holland, Amsterdam, 1973.

[EK] R. Engelking and M. Karłowicz, Some theorems of set theory and their topological consequences, Fund. Math. 57 (1965), 275-285.

[HLSh 162] B. Hart, C. Laflamme and S. Shelah, Models with second order properties, V: A General principle, Ann. Pure Appl. Logic 64 (1993), 169-194.

[MgSh 433] M. Magidor and S. Shelah, Length of Boolean algebras and ultraproducts, preprint.

[M1] D. Monk, Cardinal Invariants of Boolean Algebras, Lectures in Mathematics, ETH Zürich, Birkhäuser, Basel, 1990.

[M2] -, Cardinal Invariants of Boolean Algebras, Progr. Math. 142, Birkhäuser, Basel, 1996.

[RoSh 534] A. Rosłanowski and S. Shelah, Cardinal invariants of ultrapoducts of Boolean algebras, Fund. Math., to appear.

[RoSh 599] - - - More on cardinal functions on Boolean algebras, preprint.

[SaSh 553] O. Shafir and S. Shelah, More on entangled linear orders, preprint.

[Sh 620] S. Shelah, On measure algebra, in: Proc. Conf. Prague 1996, submitted.

[Sh 460] —, The Generalized Continuum Hypothesis revisited, Israel J. Math., submitted.

[Sh 50] -, Decomposing uncountable squares to countably many chains, J. Combin. Theory Ser. A 21 (1976), 110-114.

[Sh 345] - , Products of regular cardinals and cardinal invariants of products of Boolean algebras, Israel J. Math. 70 (1990), 129-187.

[Sh 410] —, More on Cardinal Arithmetic, Arch. Math. Logic 32 (1993), 399-428.

[Sh 371] —, Advanced: cofinalities of small reduced products, Chapter VIII of [Sh g].

[Sh 355] -, $\aleph_{\omega+1}$ has a Jonsson Algebra, Chapter II of [Sh g].

[Sh 345a] -, Basic: Cofinalities of small reduced products, Chapter I of [Sh g].

[Sh:g] —, Cardinal Arithmetic, Oxford Logic Guides 29, Oxford Univ. Press, 1994.

[Sh 400] -, Cardinal arithmetic, Chapter IX of [Sh g].

[Sh 345b] - Entangled orders and narrow Boolean algebras, Appendix 2 to [Sh g].

[Sh 405] -, Vive la différence II. The Ax-Kochen isomorphism theorem, Israel J. Math. 85 (1994), 351-390.

[Sh 430] - Further cardinal arithmetic, ibid. 95 (1996), 61-114.

[To] S. Todorčević, Remarks on chain conditions in products, Compositio Math. 5 (1985), 295-302.

Institute of Mathematics

The Hebrew University

91904 Jerusalem, Israel
Department of Mathematics

Rutgers University New Brunswick, New Jersey 08854

U.S.A.

Received 26 June 1994;

in revised form 17 December 1996 